\title{
Guidelines for Rehabilitation in Patients With Cardiovascular Disease (JCS 2012)
}

\author{
- Digest Version -
}

JCS Joint Working Group

\author{
Table of Contents \\ Introduction of the Revised Guidelines ................... 2023 \\ Abbreviations ……........................................................ 2024 \\ I Healthcare Environment Regarding Cardiac \\ Rehabilitation............................................................ 2025 \\ 1. Healthcare Costs for Cardiac Disease in Japan …...... 2025 \\ 2. Healthcare Cost and NHI Reimbursement for \\ Cardiac Rehabilitation …............................................ 2025 \\ II Effects and Mechanism of Exercise Training ... 2025 \\ 1. Physical Effects ……….......................................... 2025 \\ 2. Effects of Exercise Training on Mental Status and \\ QOL …................................................................... 2027 \\ 3. Effects of Cardiac Rehabilitation on the Secondary \\ Prevention of Cardiovascular Diseases …………......... 2028 \\ III General Rules of Exercise Training .................... 2029 \\ 1. Selection of Participants and Risk Stratification in \\ Prescribing Exercise Training Programs ………........... 2030 \\ 2. General Rules for Exercise Prescriptions ……............. 2032 \\ 3. General Guidelines for Exercise Training in the \\ Patients With Cardiovascular Diseases …………….... 2034 \\ IV Pathophysiology of Cardiovascular \\ Diseases and Exercise Training ……………........22034 \\ 1. Myocardial Infarction .................................................. 2034 \\ 2. Patients After Cardiac Surgery ……………………... 2039 \\ 3. Intervention for Angina Pectoris/Coronary Artery \\ Diseases ……………………………....................... 2041 \\ 4. Arrhythmias …....................................................... 2042 \\ 5. Acute and Chronic Heart Failure ………………......... 2046 \\ 6. Heart Transplant Recipients ………................................ 2051 \\ V Exercise Training in Children With Cardiac \\ Diseases .................................................................. 2052 \\ 1. Children After Surgery for Congenital Heart Disease .. 2052 \\ 2. Children With Congenital Heart Disease Who Have \\ Not Undergone Surgery ……………………………..... 2053 \\ 3. Problems and Future Challenges in Exercise Training \\ Programs for Children With Congenital Heart Disease … 2053 \\ VI Significance of Exercise Training in Elderly \\ Patients With Cardiovascular Diseases............. 2053 \\ 1. Significance of Exercise Training in Elderly Patients $\cdots 2053$
}

2. Exercise Training in Elderly Patients With Cardiovascular Diseases...

VII Exercise Training for Large Vessel and Peripheral Arterial Diseases................................. 2055

1. Rehabilitation in Patients With Large Vessel Diseases ……….......................................................... 2055

2. Peripheral Arterial Rehabilitation in Patients With Chronic Peripheral Arterial Occlusive Diseases.......... 2059

VIII Psychological Approaches fort the Rehabilitation in Patients With Cardiovascular Diseases …….............................. 2064

1. Importance of Clinical Psychological Interventions for Patients With Cardiovascular Diseases …………... 2064

2. Psychological Assessment of Patients With Cardiovascular Diseases.......................................... 2065

3. Psychological Interventions for Patients With Cardiovascular Diseases............................................... 2065

4. Psychological Interventions to Cardiac Rehabilitation ... 2067

IX Building an Exercise Training System .............. 2067

1. Approaches to Exercise Training Programs: How to Build Systems ……………………………………..... 2067

2. Cardiac Rehabilitation Programs and Disease Management After Discharge From Hospital ............... 2068

3. Machines, Equipment and Facilities for Exercise Training …………......................................................... 2073

X Future Prospects of Exercise Training............... 2076

1. Collaboration With Local Facilities for Exercise Training: Current Situations and Future Prospects …. 2076

2. Current Medical Service Fees for Cardiac Rehabilitation and Future Goals.................................. 2078

3. The Future of Cardiac Rehabilitation From the Health Economics Viewpoint ………………………..... 2081 XI Conclusion ……………………………………...... 2083

References …………................................................ 2083

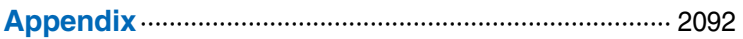

(Circ J 2014; 78: 2022-2093)

Released online July 22, 2014

Mailing address: Scientific Committee of the Japanese Circulation Society, 18F Imperial Hotel Tower, 1-1-1 Uchisaiwai-cho, Chiyoda-ku, Tokyo 100-0011, Japan. E-mail: meeting@j-circ.or.jp

This English language document is a revised digest version of Guidelines for Rehabilitation in Patients with Cardiovascular Disease reported at the Japanese Circulation Society Joint Working Groups performed in 2011 (Website: http://www.j-circ.or.jp/guideline/pdf/ JCS2012_nohara_d.pdf).

Joint Working Groups: The Japanese Circulation Society, The Japanese Coronary Association, The Japanese Association for Thoracic Surgery, The Japanese Society of Pediatric Cardiology and Cardiac Surgery, The Japanese College of Cardiology, The Japanese Association of Cardiac Rehabilitation, The Japanese Society of Electrocardiology, The Japanese Heart Failure Society, The Japanese Physical Therapy Association, The Japanese Society of Clinical Sports of Medicine

ISSN-1346-9843 doi:10.1253/circj.CJ-66-0094

All rights are reserved to the Japanese Circulation Society. For permissions, please e-mail: cj@j-circ.or.jp 


\section{Introduction of the Revised Guidelines}

The Japanese Circulation Society (JCS) has published guidelines covering many aspects of cardiovascular diseases. The JCS guidelines have become increasingly popular, and more important in clinical practice. We revised the guidelines for rehabilitation in patients with cardiovascular diseases to reflect newly obtained, evidence-based information on cardiac rehabilitation, and to describe in more detail psychological approaches to rehabilitation, secondary prevention of cardiovascular diseases, and the importance of cardiac rehabilitation in women. Because the importance of cardiac rehabilitation is not adequately understood in Japan compared to in other developed countries, physicians should be responsible for educating and encouraging patients who have cardiovascular diseases to participate in cardiac rehabilitation. We hope the present guidelines will help physicians provide appropriate rehabilitation to patients who need it.

In the present guidelines, the recommendations are classified as follows according to the system used in other JCS guidelines:

Class I: The benefits, usefulness, and efficacy of a procedure or treatment have been demonstrated in multiple multicenter randomized controlled trials (RCTs).

Class II: Some discrepancy exists in findings or opinions regarding the benefits, usefulness, and efficacy of a proce- dure or treatment.

Class IIa: The benefits, usefulness, and efficacy of a procedure or treatment have been demonstrated in a small number of multicenter RCTs.

Class IIa': Although multicenter RCTs have not been conducted, the benefits, usefulness, and efficacy of a procedure or treatment are supported by multiple observational studies or expert opinions.

Class IIb: The benefits, usefulness, and efficacy are not well demonstrated by the results of multicenter RCTs.

Class III: A procedure or treatment has been demonstrated not to be effective or useful, and possibly harmful at times, or its harmfulness has been widely agreed upon.

The level of evidence used for guideline recommendations was classified as follows:

Level of Evidence A: Data are derived from multiple multicenter RCTs in $\geq 400$ patients or in meta-analyses.

Level of Evidence B: Data are derived from multicenter RCTs in $\leq 400$ patients, well-controlled comparative studies, or large cohort studies.

Level of Evidence C: No RCTs have been conducted, but there is a consensus among the experts.

Table 1. Definition of the Phases of Cardiac Rehabilitation

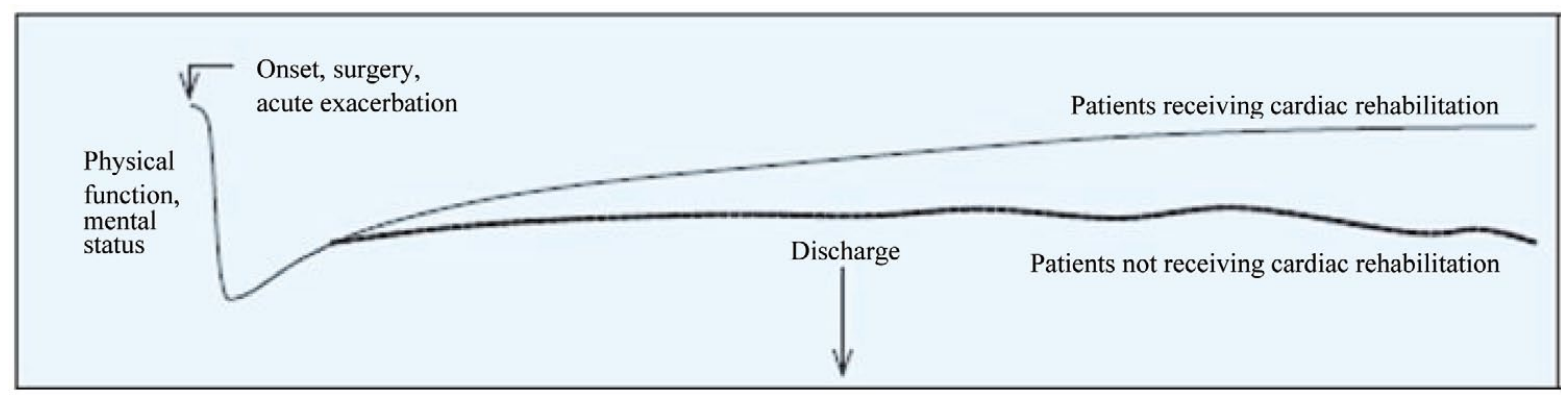

\begin{tabular}{|c|c|c|c|c|}
\hline Phase & Phase I & & Phase II & Phase III \\
\hline Timing & - Acute phase & - Early recovery phase & - Late recovery phase & - Maintenance phase \\
\hline Location & - ICU/CCU & $\begin{array}{l}\text { - General cardiovascular } \\
\text { ward }\end{array}$ & $\begin{array}{l}\text { - Outpatient/ambulatory } \\
\text { rehabilitation center }\end{array}$ & - Community exercise center \\
\hline Purpose & - Return to daily life & - Returning to society & $\begin{array}{l}\text { - Returning to society } \\
\text { - Establish new lifestyle }\end{array}$ & $\begin{array}{l}\text { - Comfortable life } \\
\text { - Prevention of recurrence }\end{array}$ \\
\hline Contents & $\begin{array}{l}\text { - Functional assessment } \\
\text { - Care planning } \\
\text { - Bedside physical } \\
\text { therapy } \\
\text { - Sitting/standing test } \\
\text { - } 30 \sim 100 \mathrm{~m} \text { walking test }\end{array}$ & $\begin{array}{l}\text { - Pathophysiological/ } \\
\text { functional assessment } \\
\text { - Mental status/psychological } \\
\text { assessment } \\
\text { - Education on the } \\
\text { importance of rehabilitation } \\
\text { - Exercise stress test } \\
\text { - Exercise prescription } \\
\text { - Instruction on ADLs, diet } \\
\text { and drug treatment } \\
\text { - Counseling } \\
\text { - How to deal with handicaps } \\
\text { - Support for return to work }\end{array}$ & $\begin{array}{l}\text { - Pathophysiological/ } \\
\text { functional assessment } \\
\text { - Mental status/psychological } \\
\text { assessment } \\
\text { - Exercise stress test } \\
\text { - Exercise prescription } \\
\text { - Exercise training } \\
\text { - Instruction on ADLs, diet and drug } \\
\text { treatment } \\
\text { - Group sessions } \\
\text { - Counseling } \\
\text { - Modification of coronary risk factors }\end{array}$ & $\begin{array}{l}\text { - Maintain better lifestyle } \\
\text { - Modification of coronary } \\
\text { risk factors } \\
\text { - Exercise prescription } \\
\text { - Exercise training } \\
\text { - Group sessions }\end{array}$ \\
\hline
\end{tabular}

$A D L$, activities of daily living; $C C U$, cardiac care unit; ICU, intensive care unit.

Adapted from Itoh $\mathrm{H}$. Chapter 6 . Recovery phase rehabilitation. In: Takano T, editor. Acute myocardial infarction, 2nd edn, Book 4 of ABC for new diagnosis and treatment. Tokyo: Saishin Igaku Sha, 2009; 278-286. ${ }^{1}$ 
In the present guideline document, the phases of cardiac rehabilitation are classified into phase I (acute phase), phase II (recovery phase), and phase III (maintenance phase) (See Table 1). ${ }^{1}$ The contents of cardiac rehabilitation differ among the phases. The duration of the acute and early recovery phases has been shortened in year 2000 and thereafter, and the lifelong prevention of secondary cardiovascular diseases has increasingly been emphasized.

\section{Abbreviations}

AAA: abdominal aortic aneurysm

ABPI: ankle-brachial pressure index

ACCF/AHA: American College of Cardiology Foundation/ American Heart Association

ACLS: advanced cardiac life support

ACSM: American College of Sports Medicine

ADL: activities of daily living

AED: automated external defibrillator

AMI: acute myocardial infarction

ASO: arteriosclerosis obliterans

ASV: adaptive servo-ventilation

AT: anaerobic threshold

AT $\dot{\mathrm{VO}}_{2}$ : oxygen uptake at anaerobic threshold

BLS: basic life support

BMI: body mass index

BNP: natriuretic peptide

BP: blood pressure

BRS: baroreflex sensitivity

CABG: coronary artery bypass grafting

CAD: coronary artery disease

CAG: coronary angiography

CCU: cardiac care unit

CHD: congenital heart disease

CK: creatine kinase

CKD: chronic kidney disease

CPAP: continuous positive airway pressure

CPX: cardiopulmonary exercise test

CRP: C-reactive protein

CRT: cardiac resynchronization therapy

CRT-D: cardiac resynchronization therapy defibrillator

CSA: central sleep apnea

CSR: Cheyne-Stokes respiration

CSR-CSA: central sleep apnea with Cheyne-Stokes respiration

CT: computed tomography

CTR: cardiothoracic ratio

eNOS: endothelial nitric oxide synthase

EOV: exertional oscillatory ventilation

HADS: Hospital Anxiety and Depression Scale

HbA1c: hemoglobin A1c

HDL: high-density lipoprotein

HOT: home oxygen therapy

HR: heart rate

HRQOL: health-related quality of life

HRVI: heart rate variability index

IABP: intra-aortic balloon pumping

ICD: implantable cardioverter defibrillator

ICT: information and communication technology

ICU: intensive care unit

KCCQ: Kansas City Cardiomyopathy Questionnaire

LDL: low-density lipoprotein
LVAD: left ventricular assist device

LVAS: left ventricular assist system

LVEF: left ventricular ejection fraction

MD: minutes distance

MDD: major depressive disorder

METs: metabolic equivalents

MHQ: Marianna Heart Failure Questionnaire

MI: myocardial infarction

MLHF: Minnesota Living with Heart Failure

MOS SF-20: Medical Outcome Study 20-item Short Form Health Survey

MOS SF-36: Medical Outcome Study 36-Item Short-Form Health Survey

MR: magnetic resonance

MRA: magnetic resonance angiography

NaSSA: noradrenergic and specific serotonergic antidepressant

NEAT: non-exercise activity thermogenesis

NGSP: National Glycohemoglobin Standardization Program

NHI: National Health Insurance

NO: nitric oxide

NYHA: New York Heart Association

OSA: obstructive sleep apnea

PAD: peripheral arterial disease

PCI: percutaneous coronary intervention

PCPS: percutaneous cardiopulmonary support

peak $\mathrm{VO}_{2}$ : peak oxygen uptake

PHQ-9: Patient Health Questionnaire-9

PVC: premature ventricular contraction

PVR: pulse volume recording

QALY: quality-adjusted life-year

RCT: randomized controlled trial

RPE: ratings of perceived exertion

SBP: systolic blood pressure

SDB: sleep-disordered breathing

SIP: Sickness Impact Profile

SNRI: serotonin and norepinephrine reuptake inhibitors

$\mathrm{SPO}_{2}$ : percutaneous arterial oxygen saturation

SSRI: selective serotonin reuptake inhibitors

SSS: sick sinus syndrome

STEMI: ST elevation myocardial infarction

TAA: thoracic aortic aneurysm

TC: total cholesterol

TG: triglyceride

TNF: tumor necrosis factor

$\mathrm{VO}_{2}$ : oxygen uptake

VWF: velocity wave form

WIQ: Walking Impairment Questionnaire

1RM: one repetition maximum 


\section{Healthcare Environment Regarding Cardiac Rehabilitation}

\section{Healthcare Costs for Cardiac Disease in Japan}

Data concerning healthcare costs and lengths of hospitalization by diagnosis in Japan indicate that patients with ischemic heart disease are decreasing in number and are hospitalized for an increasingly shorter period of time, while healthcare costs for cardiac disease are increasing. These data suggest that patients with ischemic heart disease receive intensive treatment within a short period of time. Healthcare procedures reimbursed as "fees for cardiovascular rehabilitation" in the National Health Insurance (NHI) in Japan were significantly expanded in the medical service fee revision in 2006. However, the number of patients undergoing NHI-covered cardiovascular reha- bilitation is still small.

\section{Healthcare Cost and NHI Reimbursement for Cardiac Rehabilitation}

In Japan, the cost for 1 session of rehabilitation in patients with cardiovascular diseases (cardiac rehabilitation) is estimated to be between 4,000 and 5,000 yen per person. The NHI reimbursement for cardiac rehabilitation is set to cover the cost. A questionnaire survey in 186 hospitals certified as cardiac rehabilitation providers revealed that the profits from cardiac rehabilitation vary widely among hospitals, but are surplus in most of the hospitals. ${ }^{2}$

\section{Effects and Mechanism of Exercise Training}

\section{Physical Effects}

Level of Evidence: A

1. Exercise training improves exercise capacity;

2. improves the quality of life (QOL) of patients by reducing symptoms associated with activities of daily living (ADL);

3. prevents deterioration of left ventricular systolic function and left ventricular remodeling;

4. decreases the incidence of coronary events;

5. decreases the hospitalization rate due to exacerbation of ischemic heart failure;

6. improves prognosis in patients with ischemic heart failure and coronary artery disease (CAD);

7. decreases systolic blood pressure; and

8. increases high-density lipoprotein (HDL) cholesterol levels, and decreases triglyceride levels.

Level of Evidence: B

1. Exercise training decreases heart rate and ventilatory volume at the same intensity physical activities;

2. mproves left ventricular diastolic function;

3 . is expected to reduce excessive sympathetic nervous activity;

4. prevents the progression of coronary artery atherosclerotic lesions;

5. decreases levels of inflammatory reactions such as C-reactive protein (CRP) and proinflammatory cytokines;

6. reduces platelet aggregation and blood coagulation activity; and

7. improves baroreflex sensitivity (BRS).

Level of Evidence: C

1. Exercise training decreases total peripheral vascular resistance at rest and during exercise;

2. increases maximum arteriovenous oxygen difference;

3. improves myocardial perfusion;

4. improves endothelial function in coronary arteries and peripheral arteries; and

5. increases mitochondrial density and the activities of oxidative enzymes in skeletal muscle, and promotes the conversion from type II to type I muscle fiber.
Exercise training has been demonstrated to improve the physical effects listed in Table 2. ${ }^{3-38}$ In patients with chronic CAD, cardiac rehabilitation may be as effective as drug treatment and catheter intervention in terms of the improvement of prognosis. ${ }^{6,57}$

\section{Increase in Exercise Capacity}

Improvement in exercise capacity is one of the most reliable effects of exercise training, and it has been reported that the peak oxygen uptake (peak $\mathrm{VO}_{2}$ ) as an index of exercise capacity, increases by 15 25\% after exercise training in patients with cardiovascular diseases. ${ }^{3-34}$ As a relative intensity of the same physical activity is reduced, exercise training alleviates exercise-induced symptoms, such as shortness of breath and anginal pain, associated with daily activities. The improvement in exercise capacity is assumed to be due to an increase in myocardial ischemic threshold in patients with $\mathrm{CAD}^{6,23,24}$ and improvements in peripheral effects such as peripheral circulation and skeletal muscle function in patients with chronic heart failure. $11,21,22$

\section{Increase in Muscle Strength After Resistance Training}

Relatively low intensity resistance training has been established to be safe in patients with cardiovascular diseases. Resistance training is expected to enhance the strength of major muscle groups, facilitate ADLs, and improve the QOL of patients with chronic heart failure and low muscle strength..$^{58}$

3. Effects on Cardiac Function and Ventricular Remodeling It has been demonstrated that exercise training does not deteriorate left ventricular remodeling, but rather decreases left ventricular end-diastolic volume and improves exercise capac-

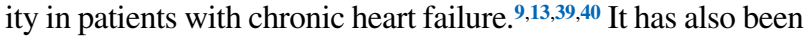
suggested that exercise training may prevent postinfarction ventricular remodeling. ${ }^{9}$ An increasing number of reports have described that exercise training improves left ventricular diastolic function, ${ }^{17,18,39}$ and also improves left ventricular systolic function slightly. ${ }^{12,39-41}$

\section{Effects on Coronary Circulation}

Exercise training increases the myocardial ischemic threshold by improving myocardial perfusion. ${ }^{23,24,28}$ It has been pointed out that decreased coronary vasodilator reserve plays a role in 


\begin{tabular}{|c|c|c|}
\hline Category & Effects & Rank \\
\hline \multirow[t]{2}{*}{ Exercise capacity } & Increase peak uptake & $A^{3-34}$ \\
\hline & Increase anaerobic threshold & $A^{4,11,31,33}$ \\
\hline \multirow[t]{2}{*}{ Symptoms } & Decrease the incidence of anginal attacks by increasing myocardial ischemic threshold & $A^{23,24,28}$ \\
\hline & Reduce symptoms of heart failure associated with the same intensity physical activities & $A^{4,7,21,31}$ \\
\hline Respiration & Decrease ventilatory volume at given submaximal workloads & $A^{30,31,33}$ \\
\hline \multirow[t]{6}{*}{ Heart } & Decrease heart rate at given submaximal workloads & $A^{11-23}$ \\
\hline & Decrease cardiac work (double product) at given submaximal workloads & $A^{23}$ \\
\hline & Prevent left ventricular remodeling & $A^{9-13,39-41}$ \\
\hline & Prevent the deterioration of left ventricular systolic function & $A^{9-13,39-41}$ \\
\hline & Improve left ventricular diastolic function & $\mathrm{B}^{17,18,39}$ \\
\hline & Improve myocardial metabolism & $\mathrm{B}^{19,20}$ \\
\hline \multirow[t]{3}{*}{ Coronary arteries } & Prevent the progression of coronary atherosclerotic lesions & $A^{24-27}$ \\
\hline & Improve myocardial perfusion & $\mathrm{B}^{23,24,28}$ \\
\hline & Improve endothelium-dependent and -independent vasodilation responses & $B^{29,42}$ \\
\hline Peripheral oxygen utilization & Increase the maximum arteriovenous oxygen difference & $\mathrm{B}^{21,22}$ \\
\hline \multirow[t]{2}{*}{ Peripheral circulation } & Decrease total peripheral vascular resistance at rest and during exercise & $\mathrm{B}^{11,21}$ \\
\hline & Improve peripheral arterial endothelial function & $B^{43-45}$ \\
\hline Inflammatory reactions & Decrease CRP and proinflammatory cytokines & $B^{46-48}$ \\
\hline \multirow[t]{4}{*}{ Skeletal muscle } & Increase mitochondrial density & $B^{49,50}$ \\
\hline & Increase the activity of oxidative enzymes in skeletal muscle & $\mathrm{B}^{38,39}$ \\
\hline & Increase capillary density in skeletal muscle & $\mathrm{B}^{38,39}$ \\
\hline & Promote the conversion from type II to type I muscle fiber & $\mathrm{B}^{38,39}$ \\
\hline \multirow[t]{3}{*}{ Coronary risk factors } & Decrease systolic blood pressure & $A^{3,35,38,51}$ \\
\hline & Increase HDL cholesterol and decrease triglycerides leveles & $A^{3,35,38,51}$ \\
\hline & Decrease smoking rate & $\mathrm{A}^{3,35,38}$ \\
\hline \multirow[t]{3}{*}{ Autonomic nervous system } & Reduce the sympathetic nervous activity & $A^{7,52,53}$ \\
\hline & Increase parasympathetic nervous activity & $\mathrm{B}^{7,52,53}$ \\
\hline & Improve the baroreflex sensitivity & $\mathrm{B}^{52}$ \\
\hline \multirow[t]{2}{*}{ Blood } & Reduce platelet aggregation & $\mathrm{B}^{54}$ \\
\hline & Reduce blood coagulation & $\mathrm{B}^{55,56}$ \\
\hline \multirow[t]{3}{*}{ Prognosis } & Reduce the incidence of coronary events & $A^{5,6,35,36}$ \\
\hline & Reduce hospitalization rate due to exacerbation of heart failure & $A(C A D)^{4,37}$ \\
\hline & Improve prognosis (reduce all-cause mortality and cardiac death) & $A(C A D)^{4-6,35-37}$ \\
\hline
\end{tabular}

A, supported by substantial evidence; $B$, supporting reports are high in quality but limited in number; CAD, coronary artery disease; CRP, C-reactive protein; HDL, high-density lipoprotein.

the development of myocardial ischemia. Exercise training may enhance coronary perfusion by improving endotheliumdependent and -independent vasodilation responses even if coronary artery lesions remain unchanged. ${ }^{29}$ Studies of comprehensive programs, including diet therapy, and exercise training, revealed that these programs not only prevented the progression of coronary artery lesions but also induced regression of coronary artery lesions, and decreased the incidence of coronary events. The favorable effects of exercise training only without other intervention have also been reported. ${ }^{24-27}$

\section{Improvement in Ventilatory Function}

Exercise training reduces excessive ventilation and dyspnea by decreasing afferent nerve stimulation from skeletal muscles and enhancing respiratory muscle function. ${ }^{30-34}$

\section{Improvement in Autonomic Nervous System Function}

Patients with cardiovascular diseases are prone to exhibit persistent sympathetic nervous activity, which contributes to the progression of heart failure and the occurrence of severe arrhythmia. Exercise training reduces excessive sympathetic nervous activity, and enhances parasympathetic nervous activity. ${ }^{7,52,53}$

\section{Effects on Peripheral Circulation}

In patients with chronic heart failure, blood flow in skeletal muscle does not increase appropriately during exercise. This is a major factor causing impaired exercise capacity, ${ }^{11}$ and is suspected to be due to vascular endothelial dysfunction. Exercise training improves vascular endothelial function. ${ }^{43}$

\section{Improvement of Inflammatory Reactions}

Proinflammatory reactions significantly participate in the pathophysiology of atherosclerosis and chronic heart failure. Exercise training has anti-inflammatory effects, and decreases levels of CRP and proinflammatory cytokines. ${ }^{46-48}$

\section{Adaptation of Skeletal Muscle to Exercise}

Exercise training increases the capillary density of skeletal muscle, promotes a conversion from type II to type I muscle fiber, 
and increases the number and oxidative enzyme activities of mitochondria. ${ }^{47}$ These peripheral mechanisms play important roles in the increase in peak $\dot{\mathrm{VO}}_{2}$ by exercise training.

\section{Modification of Coronary Risk Factors}

Exercise training is effective in modifying coronary risk factors. Comprehensive programs including exercise training have been confirmed to be effective in decreasing blood pressure, improving lipid metabolism and glucose tolerance, and promoting smoking cessation. ${ }^{3,35,38,59}$ Exercise training also decreases platelet aggregation and blood coagulation activity. ${ }^{54-56}$

\section{Improvement of Prognosis}

It has been reported that exercise training improves the prognosis of patients with CAD and ischemic heart failure by decreasing the incidences of coronary events and hospitalizations for exacerbations of heart failure..$^{4,35-37}$

\section{Sex Differences in the Effects of Exercise Training}

It is known that women tend not to participate in cardiac rehabilitation, ${ }^{60-63}$ and the incidence, severity, and duration of depression after myocardial infarction (MI) are higher in female than in male patients. ${ }^{64}$ Cardiac rehabilitation programs for women should be developed and the effects of such programs in women should be evaluated. ${ }^{61}$

\section{Effects of Exercise Training on Mental Status and QOL}

\section{Effects of Exercise Training on Depression}

\section{Class I}

None.

\section{Class Ilb}

1. Exercise training should be considered for CAD or chronic heart failure patients with depression. (Level of Evidence: C)

It has been reported that depression is common among patients with cardiovascular diseases, and the severity of the depression increase as worsening difficulty to perform ADLs especially in patients with chronic heart failure. ${ }^{65}$ It is unclear whether depression directly affects prognosis in patients with CAD or chronic heart failure. Because few studies have conducted depression as the main outcome in investigating the effects of cardiac rehabilitation, further research regarding the efficacy of cardiac rehabilitation on depression will need.

\section{Effects of Exercise Training on QOL}

\section{Class I}

1. Exercise training is strongly recommended to improve the QOL in patients after MI or coronary artery bypass grafting (CABG). (Level of Evidence: A)

2. Exercise training is also strongly recommended to improve the QOL in patients with chronic heart failure. (Level of Evidence: A)

\section{Class Ilb}

1. The improvement in QOL by exercise training differs substantially by the severity of cardiovascular diseases, and the mode, intensity and duration of exercise training. (Level of Evidence: C)
The one of major purpose of cardiac rehabilitation is to improve health-related quality of life (HRQOL) in patients with cardiovascular diseases.

\section{How to Assess QOL}

QOL questionnaires frequently used in the cardiology field include the Sickness Impact Profile (SIP), ${ }^{66}$ the MacMaster Health Index Questionnaire, ${ }^{67}$ the Nottingham Health Profile, ${ }^{68}$ and the Medical Outcome Study (MOS) 36-Item Short-Form Health Survey (SF-36). ${ }^{69}$ Disease specific QOL questionnaires for cardiovascular diseases include the Minnesota Living with Heart Failure (MLHF) Questionnaire ${ }^{70}$ and the Kansas City Cardiomyopathy Questionnaire (KCCQ). ${ }^{71}$ In Japan, the Marianna Heart Failure Questionnaire (MHQ) ${ }^{72}$ was developed as an evaluation of symptoms due to heart failure. It is recommended to apply disease specific QOL questionnaires in this patient population, because commonly used QOL measure such as SF-36 does not sufficiently consider the clinical features of cardiovascular diseases.

\section{Effects of Cardiac Rehabilitation on QOL of Patients With Cardiovascular Diseases}

The evidence has been established by a large number of RCTs which reported the favorable effects of cardiac rehabilitation on QOL in patients with CAD, including acute myocardial infarction (AMI) or CABG. The effects of exercise training on QOL in patients with chronic heart failure have also been reported in many RCTs, and evidence is being accumulated rapidly. While the effects of cardiac rehabilitation on the QOL has been demonstrated in chronic heart failure patients with systolic dysfunction only a few reports have described in chronic heart failure patients with preserved ejection fraction. Further studies needs to clarify in this regard. In Japan, it is expected that the number of the patients with heart transplantation or left ventricular assist device (LVAD) gradually increase and perform cardiac rehabilitation, so data on the effects of cardiac rehabilitation in these patient populations will need to be accumulated in the future.

There is also little evidence regarding the effect of cardiac rehabilitation on QOL in patients with peripheral vascular diseases. Further studies are required.

\section{Methodology of Cardiac Rehabilitation to Improve QOL}

The effects of comprehensive programs, exercise plus lifestyle modification, as well as exercise training on QOL have been reported. Studies of cardiac rehabilitation in patients with CAD and chronic heart failure have been conducted mainly performing programs combining exercise training and psychosocial approaches, however the results of these programs are controversial.

Regarding the type of exercise training, it has been well documented that a combination of aerobic exercise and resistance training is better than aerobic exercise alone in terms of the improvement in QOL. Recently, improvement in QOL has been reported in high-intensity interval training ${ }^{40,73-75}$ and electrical muscle stimulation therapy in patients with severe heart failure. ${ }^{76}$ Further studies are required to establish evidence.

Almost all studies with successful results of the improvement in QOL performed cardiac rehabilitation for at least 16 weeks, suggesting that intervention period might be one of the determinants to obtain improvement in QOL. 
Table 3. Goals for Blood Pressure Control

\section{Primary prevention}

Secondary prevention of coronary artery disease

Patients with diabetes (including impaired glucose tolerance) or CKD

CKD, chronic kidney disease.

Source: the Guidelines for the Primary Prevention of Ischemic Heart Disease (JCS 2006) ${ }^{92}$ and the Guidelines for Secondary Prevention of Myocardial Infarction (JCS 2011) ${ }^{93}$ published by the Japanese Circulation Society, and the Japanese Society of Hypertension Guidelines for Management of Hypertension (JSH 2009) ${ }^{94}$ published by the Japan Society of Hypertension.

Table 4. Goals for Lipid Control

Target blood pressure levels $<140 / 90 \mathrm{mmHg}$

$<130 / 80 \mathrm{mmHg}$ at clinic

$<125 / 75 \mathrm{mmHg}$ in home $<130 / 80 \mathrm{mmHg}$

\begin{tabular}{l}
$\quad$ Treatment rules / Category \\
\cline { 2 - 2 } Primary prevention: Modify lifestyle, then consider the indication for drug treatment
\end{tabular}

\section{Effects of Cardiac Rehabilitation on the Secondary Prevention of Cardiovascular Diseases}

\section{Improvement of Prognosis by Exercise Training and Its Mechanism}

\section{Class I}

1. Exercise training is expected to decrease all-cause mortality in patients with CAD; (Level of Evidence: A)

2. decreases cardiac mortality in patients with CAD; (Level of Evidence: A) and

3. decreases the incidence of recurrent fatal MI. (Level of Evidence: A)

\section{Class Ila}

1. Exercise training is expected to increase heart rate variability and BRS by enhancing parasympathetic nervous activity, and to reduce excessive sympathetic nervous activity and heart rate. (Level of Evidence: B)

\section{Class Ila'}

1. Exercise training is expected to prevent plaque rupture by stabilizing coronary atherosclerotic lesions; (Level of Evidence: C) and
2. alleviates the inflammation that drives the progression of coronary atherosclerosis. (Level of Evidence: C)

Meta-analyses of studies that evaluated the effects of exercise training on the secondary prevention of CAD and on improving in short-term mortality of prognosis have provided Level A scientific evidence.,5,77,78 However, conclusive Level A evidence has not been obtained in terms of long-term ( $\geq 10$-year) prognosis (Level of Evidence: B) ${ }^{79}$ In addition to the modifying of coronary risk factors, exercise training has been reported to improve prognosis by stabilizing coronary atherosclerotic lesions, and favorably affecting endothelial function and autonomic function (Level of Evidence: B) ${ }^{6,78,80}$ No strong evidence has been found in terms of whether exercise training may prevent plaque rupture by stabilizing coronary atherosclerotic lesions and alleviating inflammation to slow or prevent the progression of coronary atherosclerosis. ${ }^{81-84}$

\section{Modification of Atherosclerotic Risk Factors \\ Class I}

1. Cardiac rehabilitation is expected to exert mild antihypertensive effect. (Level of Evidence: A)

2. Cardiac rehabilitation is expected to improve lipid profile. (Level of Evidence: $\mathrm{A}$ )

3. Comprehensive programs including long-term diet therapy 
Table 5. Goals for Body Weight Control

Primary prevention

Secondary prevention
$\mathrm{BMI}^{*}<25.0 \mathrm{~kg} / \mathrm{m}^{2}$

Waist circumference: $<85 \mathrm{~cm}$ for males, $<90 \mathrm{~cm}$ for females

$\mathrm{BMI}^{*} 18.5 \sim 24.9 \mathrm{~kg} / \mathrm{m}^{2}$

Waist circumference: $<85 \mathrm{~cm}$ for males, $<90 \mathrm{~cm}$ for females

${ }^{*} \mathrm{BMI}$, body mass index (calculated as body weight $[\mathrm{kg}] \div[\text { height in meters }]^{2}$ )

Source: the Guidelines for the Primary Prevention of Ischemic Heart Disease (JCS 2006) ${ }^{92}$ and the Guidelines for

Secondary Prevention of Myocardial Infarction (JCS 2011) ${ }^{93}$ published by the Japanese Circulation Society.

Table 6. Goals for Diabetes Management

Primary prevention

Secondary prevention
Absence of diabetes (including impaired glucose tolerance)

$\mathrm{HbA1c}(\mathrm{NGSP})<7.0 \%$

HbA1c, hemoglobin A1c; NGSP, National Glycohemoglobin Standardization Program.

Source: the Guidelines for the Primary Prevention of Ischemic Heart Disease (JCS 2006) ${ }^{92}$ and the Guidelines for

Secondary Prevention of Myocardial Infarction (JCS 2011) (33 $^{93}$ published by the Japanese Circulation Society.

\begin{tabular}{lcccc|}
\hline \begin{tabular}{l} 
Table 7. Alcohol Content of Beverages \\
\multicolumn{1}{c}{ Beverages }
\end{tabular} & $\begin{array}{c}\text { Alcohol content } \\
(\%)\end{array}$ & Serving size & $\begin{array}{c}\text { Volume } \\
(\mathbf{m L})\end{array}$ & $\begin{array}{c}\text { Ethanol content } \\
(\mathbf{m L})\end{array}$ \\
Beer, low-malt beer & 5 & 1 can & 350 & 17.5 \\
Wine & 15 & A wine glass & 110 & 16.5 \\
Shochu (distilled spirits) & 25 & $\begin{array}{c}\text { A glass of shochu mixed } \\
\text { with hot water }(1: 1)\end{array}$ & 90 & 22.5 \\
Sake & 15 & 180 mL & 180 & 27.0 \\
Whiskey, brandy & 40 & A single shot & 30 & 12.0 \\
Chinese rice wine & 17 & A small glass & 50 & 8.5 \\
\hline
\end{tabular}

Ethanol content=Volume of beverage $(\mathrm{mL}) \times($ alcohol content $[\%] \div 100)$.

are expected to improve body weight control. (Level of Evidence: A)

4. Combination of diet therapy and exercise training is expected to decrease the incidence of cardiovascular events in patients with insulin-dependent diabetes. (Level of Evidence: A)

\section{Class II}

1. Patient education is expected to be helpful in encouraging smoking cessation and body weight control. (Level of Evidence: A)

Modification of atherosclerotic risk factors such as hypertension, ${ }^{25,35,85,86}$ diabetes,${ }^{87,88}$ and dyslipidemia ${ }^{35,89,90}$ (i.e., hyperlow-density lipoprotein [LDL] cholesterolemia and hypo- HDL cholesterolemia) is important to improve prognosis, and Level A evidence on the effects of exercise training on these risk factors has been accumulated. However, conventional exercise training programs exert short-term effects on body weight control and smoking cessation, ${ }^{25}$ but are not sufficient to en- sure long-term modification of risk factors. Because women tend not to continue cardiac rehabilitation, long-term comprehensive programs that include appropriate patient education should be developed. ${ }^{91}$ It has become aware that comprehensive programs improve prognosis by modifying not only atherosclerotic risk factors but also multiple risk factors. ${ }^{80,89}$

Recommendations for modification of atherosclerotic risk factors are summarized as follows:

1) Hypertension (Table 3) $)^{92-94}$

2) Dyslipidemia (Table 4) ${ }^{95}$

3) Smoking; complete smoking cessation and avoidance of passive smoking are essential in primary and secondary prevention of cardiovascular diseases.

4) Obesity; (body weight control) (Table 5) 92,93 $^{9}$

5) Diabetes (Table 6) ${ }^{92,93}$

6) Patient education

7) Alcoholic beverages; abstinence or a restriction to light alcohol consumption $(20 \sim 30 \mathrm{~mL}$ ethanol per day for men, $10 \sim 20 \mathrm{~mL}$ ethanol per day for women) should be encouraged (Table 7).

\section{General Rules of Exercise Training}

\section{Class I}

1. Prior to beginning exercise training programs, participants should be assessed for clinical status and undergo examinations at rest and exercise stress tests to determine the ap- propriateness of exercise training and to establish the appropriate exercise prescriptions. (Level of Evidence: A)

2. Exercise training should be considered as a measure to treat lifestyle-related diseases that increase the risk of CAD. 


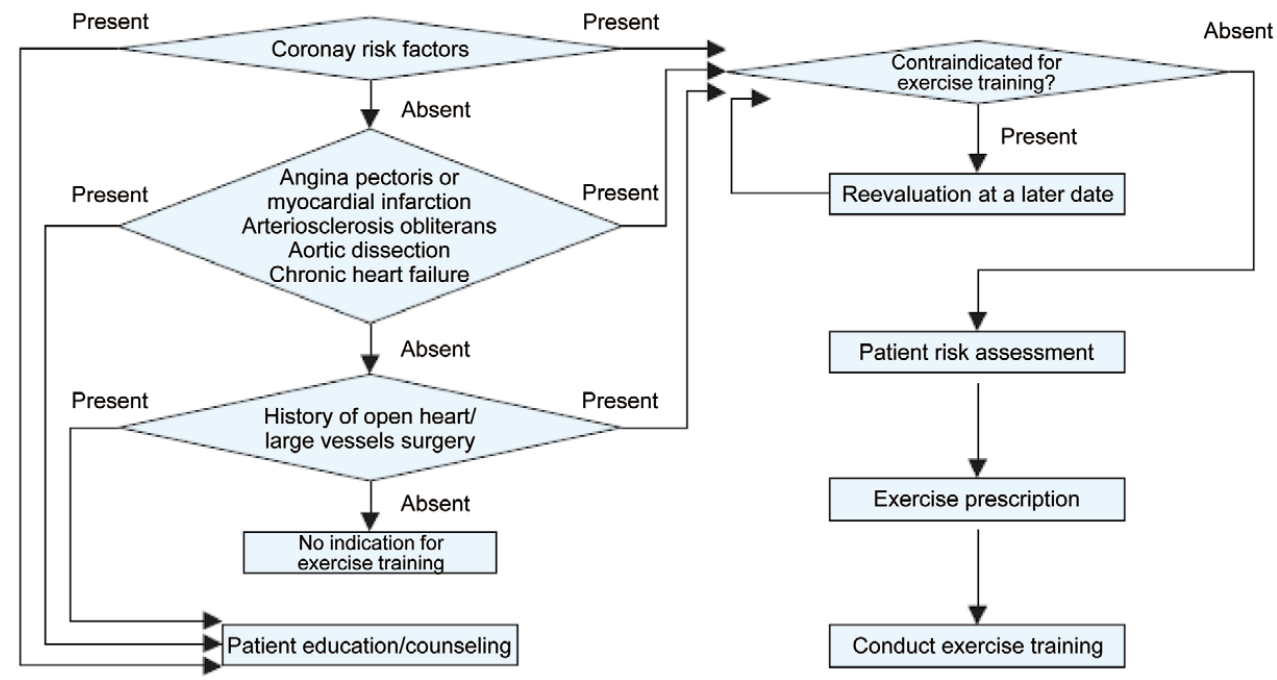

Figure 1. Flowchart for exercise training programs. The following flowchart describes how to assess whether exercise training programs are indicated or contraindicated for healthy individuals and patients with cardiovascular diseases.

\section{(Level of Evidence: A)}

3. Exercise training should be considered as an intervention for patients with CAD, including angina pectoris and MI. (Level of Evidence: A)

The following sections describe important considerations in exercise prescriptions for healthy individuals and patients with cardiovascular diseases in terms of selection of participants, risk stratification, medical check-ups, general rules in prescribing exercise prescriptions, and precautions of exercise training. Figure 1 shows a flowchart of exercise training for patients with cardiovascular diseases.

\section{Selection of Participants and Risk Stratification in Prescribing Exercise Training Programs}

To provide effective and safe exercise prescriptions for healthy individuals, individuals who have risk factors for atherosclerosis, and patients with cardiovascular diseases, physicians should select participants appropriately on the basis of medical history, physical findings, and medical evaluation. Participants should be stratified by risk according to the severity of cardiovascular diseases and presence/absence of noncardiovascular complications.

\section{Medical Check-ups for Exercise Training (Table 8) ${ }^{96}$}

Prior to beginning exercise training programs, candidates, including apparently healthy individuals, should be assessed for clinical status and undergo examinations at rest and exercise stress tests to determine the appropriateness of exercise training for individual participants and to establish the appropriate exercise prescription. Clinical status should be assessed through medical interviews about subjective symptoms, medical history, family history, and lifestyle. Medical check-ups should include measurement of blood pressure, pulse rate, electrocardiographic activity. ${ }^{96}$ Levels of blood glucose, total cholester- ol, triglycerides, hepatic enzymes, and body mass index should also be evaluated. ${ }^{97}$

\section{Exercise Stress Test (Tables 9 and 10$)^{98}$}

Exercise stress test is important in determining whether exercise training is indicated for individual patients. The present guideline document does not describe the indications and methods of exercise stress test in detail. In brief, exercise stress test, as part of the medical check-ups conducted prior to the initiation of exercise training, is necessary for patients with cardiac disease, patients with signs/symptoms of cardiovascular diseases such as chest pain, shortness of breath, and intermittent claudication, and patients with coronary risk factors such as diabetes and dyslipidemia. ${ }^{99}$

\section{Exercise Training for Patients With Lifestyle-Related Diseases and Metabolic Syndrome (Table 11)}

Exercise training is effective in the treatment of lifestyle-related diseases. ${ }^{100,101}$ Comprehensive programs including exercise training and diet therapy are important for the treatment of hypertension, diabetes, and dyslipidemia. After the definition of the concept and diagnostic criteria of metabolic syndrome as the basis of these lifestyle-related diseases, the "Exercise and Physical Activity Reference for Health Promotion 2006 (EPAR 2006): Physical Activity, Exercise, and Physical Fitness" and the "Exercise and Physical Activity Guide for Health Promotion $2006 \sim$ To Prevent Lifestyle-related Diseases $\sim<$ Exercise and Physical Activity Guide for Health Program 2006>" were published by the Office for Lifestyle-related Diseases Control, General Affairs Division, Health Service Bureau, the Ministry of Health, Labor and Welfare (MHLW) of Japan. ${ }^{102,103}$

\section{Exercise Training for Patients With Ischemic Heart Disease}

Patients after AMI account for a large population of patients receiving exercise training as rehabilitation after CAD. Exercise training for patients after AMI is generally classified into three phases: phase I (acute phase) during intensive care unit 
Table 8. Basic Medical Information Required to Prescribe Exercise Training Programs

Basic medical information_ Exercise stress test

Other measures

\section{Symptoms}

Chest pain, chest discomfort, palpitation, shortness of breath

Dizziness, syncope, intermittent claudication

Spine symptoms, joint symptoms

Exercise stress test

Medical history

Cardiovascular diseases

Required

Required

Orthopedic examination/ consultation

Orthopedic diseases

Required

\section{Lifestyle-related diseases}

Hypertension

Diabetes

Dyslipidemia

Obesity

Family history*

Myocardial infarction or sudden death in first-degree relatives

Lifestyle

Exercise, diet, smoking, drinking

ECG at rest

Myocardial infarction

Abnormal ST-T

Ventricular arrhythmia

Other important findings

$\begin{array}{cc}\text { Required } & \\ & \text { Orthopedic examination/ cons } \\ \text { See hypertension in Table 11 } & \text { Severity assessment } \\ \text { See diabetes in Table 11 } & \text { Severity assessment } \\ \text { See dyslipidemia in Table 11 } & \text { Severity assessment } \\ \text { See obesity in Table 11 } & \text { Severity assessment }\end{array}$

Required

Careful assessment is required for patients whose first-degree relative(s) experienced cardiovascular events at a relatively younger age, i.e. patients whose father or other first-degree male relative or mother or other first-degree female experienced myocardial infarction, coronary revascularization, or sudden death at $<55$ and $<65$ years of age, respectively.

Adapted from Japan Medical Association. Exercise training prescription manual. Japan Medical Association Journal 1996; 116(Suppl): $1-49,{ }^{96}$ with modification.

Table 9. Contraindications for Exercise Stress Test

\section{Absolute contraindications}

1. Acute myocardial infarction developed within 2 days

2. Unstable angina not controlled with medical treatment

3. Uncontrolled arrhythmia that cause symptoms or hemodynamic compromise

4. Symptomatic severe aortic stenosis

5. Uncontrolled symptomatic heart failure

6. Acute pulmonary embolism or pulmonary infarction

7. Acute myocarditis or pericarditis

8. Acute aortic dissection

9. Mental disorders associated with communication difficulties

Relative contraindications

1. Left main coronary artery stenosis

2. Moderate stenotic valvular heart disease

3. Electrolyte abnormality

4. Severe hypertension*

5. Tachyarrhythmia or bradyarrhythmia

6. Hypertrophic cardiomyopathy or other outflow tract obstruction

7. Mental or physical impairment leading to inability to exercise adequately

8. Advanced atrioventricular block

*It is recommended that severe hypertension is suggested as a systolic blood pressure of $>200 \mathrm{mmHg}$ and/or a diastolic blood pressure of $>110 \mathrm{mmHg}$, in principle.

Adapted from Gibbons RJ, et al. ACC/AHA Guidelines for Exercise Testing. J Am Coll Cardiol 1997; 30: 260-311,98 with permission from Elsevier Inc., modified for Japanese patients.

/cardiac care unit (ICU/CCU) treatment; phase II (recovery phase) consisting of the early recovery phase in the general cardiovascular ward to discharge from hospital, and the late recovery phase from immediately after discharge to returning to work; and phase III (maintenance phase) after returning to work. However, in Japan, the recovery phase is defined as the 
Table 10. Criteria for Terminating Exercise Test
1. Symptoms
Anginal pain, dyspnea, syncope, dizziness, light-headed feeling, leg pain (claudication)
2. Signs
Cyanosis, facial pallor, cold sweat, ataxia
3. Blood pressure Insufficient increase or progressive decrease in systolic blood pressure during exercise, or abnormal increase in blood pressure $(\geq 225 \mathrm{mmHg})$
4. ECG Apparent ischemic ST-T changes, cardiac rhythm disorder (e.g., severe tachycardia or bradycardia, ventricular tachycardia, frequent arrhythmias, atrial fibrillation, $\mathrm{R}$ on $\mathrm{T}$, and premature ventricular contractions), and second or third degree atrioventricular block

Source: Gibbons RJ, et al. ACC/AHA Guidelines for Exercise Testing. J Am Coll Cardiol 1997; 30: 260-311.98

\begin{tabular}{|c|c|c|c|}
\hline Diagnosis & Indications & Conditional indications & Contraindications \\
\hline Hypertension & $140 \sim 159 / 90 \sim 94 \mathrm{mmHg}$ & $\begin{array}{l}\text { BP of } 160 \sim 179 / 95 \sim 99 \mathrm{mmHg} \text {; ongoing } \\
\text { antihypertensive treatment with BP less than the } \\
\text { contraindicated level; men over } 40 \text { years and } \\
\text { women over } 50 \text { years should undergo exercise } \\
\text { stress tests whenever possible. If exercise } \\
\text { stress tests are not feasible, prescribe walking } \\
\text { or other light exercise. }\end{array}$ & $\begin{array}{l}\text { BP of } \geq 180 / 100 \mathrm{mmHg} \text {; chest } X \text {-ray findings } \\
\text { with a CTR of } \geq 55 \% \text {; ECG indicating severe } \\
\text { arrhythmia or ischemic changes (excluding } \\
\text { those with favorable results of exercise stress } \\
\text { tests); hypertensive changes (Ilb or higher) on } \\
\text { funduscopy; urinary protein of } \geq 100 \mathrm{mg} / \mathrm{dL}\end{array}$ \\
\hline Diabetes & $\begin{array}{l}\text { Fasting blood glucose } \\
\text { level: } 110 \sim 139 \mathrm{mg} / \mathrm{dL}\end{array}$ & $\begin{array}{l}\text { Fasting blood glucose level of } 140 \sim 249 \mathrm{mg} / \mathrm{dL} \text {; } \\
\text { ongoing antidiabetic treatment with glucose } \\
\text { levels less than the contraindicated level; men } \\
\text { over } 40 \text { years and women over } 50 \text { years should } \\
\text { undergo exercise stress tests whenever } \\
\text { possible. If exercise stress tests are not feasible, } \\
\text { prescribe walking or other light exercise. }\end{array}$ & $\begin{array}{l}\text { Fasting blood glucose level of } \geq 250 \mathrm{mg} / \mathrm{dL} \text {; } \\
\text { positive urinary ketones; diabetic retinopathy }\end{array}$ \\
\hline Dyslipidemia & $\begin{array}{l}\text { TC: } 220 \sim 249 \mathrm{mg} / \mathrm{dL} \text { or } \\
\text { TG: } 150 \sim 299 \mathrm{mg} / \mathrm{dL}\end{array}$ & $\begin{array}{l}\mathrm{TC} \geq 250 \mathrm{mg} / \mathrm{dL} \text { or } \mathrm{TG} \geq 300 \mathrm{mg} / \mathrm{dL} \text {, ongoing lipid } \\
\text { management; men over } 40 \text { years and women } \\
\text { over } 50 \text { years should undergo exercise stress } \\
\text { tests whenever possible. If exercise stress tests } \\
\text { are not feasible, prescribe walking or other light } \\
\text { exercise. }\end{array}$ & \\
\hline Obesity & BMI: 24.0 29.9 & $\begin{array}{l}\text { BMI: 24.0 29.9, detailed assessment for leg } \\
\text { joint disorder; limitation of exercise }\end{array}$ & BMI: $\geq 30.0$ \\
\hline
\end{tabular}

BMI, body mass index (calculated as body weight [kg] $\div[$ height in meters]2); BP, blood pressure; CTR, cardiothoracic ratio; TC, total cholesterol; TG, triglycerides.

Adapted from Japan Medical Association. Exercise training prescription manual. Japan Medical Association Journal 1996; 116(Suppl): 1-49,96 with modification.

first 5 months of treatment during which costs for cardiac rehabilitation are covered by the NHI, and the maintenance phase is defined as month 6 and thereafter, regardless of whether the patient achieved returning to work. The reader should refer to other sections of this document for the contents of exercise training for patients with CAD.

\section{Exercise Training for Patients With Other Types of Cardiovascular Diseases}

In the revision of the NHI reimbursement in April 2005, indications of "cardiovascular rehabilitation" were expanded to include cardiac rehabilitation programs provided in medical institutions registered to the relevant social insurance office for patients with large vessel diseases (e.g., aortic dissection, after large vessel surgery), chronic heart failure (left ventricular ejection fraction [LVEF] of $\leq 40 \%$; peak $\dot{\mathrm{VO}}_{2}$ of $\leq 80 \%$ of the reference value; or brain natriuretic peptide $[\mathrm{BNP}]$ of $\geq 80 \mathrm{pg} / \mathrm{mL}$ ), and patients with peripheral arterial occlusive diseases complicated with intermittent claudication, in addition to the conventional three indications of patients with angina pectoris, those with MI, and those who have had open heart surgery. The reader should refer to Chapter IV-5 "Acute and Chronic Heart Failure" and Chapter VII "Exercise Training for Large Vessel and Peripheral Arterial Diseases".

\section{Risk Stratification for Complications During Exercise Training}

The indications of cardiac rehabilitation are expanding, and a wide variety of patients are being introduced to exercise training programs. Because the response to exercise training may vary among patients, healthcare professionals should understand the clinical status of individual patients to provide individualized training programs appropriate for each patient. The American Heart Association (AHA) stratifies patients undergoing exercise training according to symptoms and cardiac function, and recommends activity guidelines, supervision, and ECG monitoring for stratified participants according to the risk stratification (Table 12). ${ }^{99}$ This classification may also be applied to patients in Japan.

\section{General Rules for Exercise Prescriptions}

Exercise prescriptions are developed to improve physical condition by enhancing exercise capacity and modifying coronary risk factors, and to confirm the safety of exercise training. Exercise prescriptions consist of (1) the type of exercise, (2) exercise intensity, (3) duration of exercise, and (4) the frequency of exercise, and (5) are modified after improvement in 
Table 12. Indications, Contraindications, and Risk Classification for Exercise Training

Cardiovascular conditions and clinical findings

\section{Class A: Apparently healthy individuals}

1. Men $<45$ years and women $<55$ years who have no symptoms or known presence of coronary risk factors

2. Men $\geq 45$ years and women $\geq 55$ years who have no symptoms or known

3. Men $\geq 45$ years or women $\geq 55$ years who have no symptoms or known presence of cardiac disease and with $\geq 2$ cardiovascular risk factors presence of cardiac disease and with $<2$ cardiovascular risk factors

Limitations and monitoring

Activity guidelines: No restrictions.

Supervision required: None.

ECG and blood pressure monitoring: Not required

Class B: Presence of known, stable cardiovascular disease with low risk for complications with vigorous exercise, but slightly greater than for apparently healthy individuals

This classification includes individuals with any of the following diagnoses:

1. Stable coronary artery disease

2. Mild or moderate valvular heart disease, excluding severe valvular stenosis or regurgitation

3. Congenital heart disease

4. Cardiomyopathy: ejection fraction $\leq 30 \%$; excluding hypertrophic cardiomyopathy or recent myocarditis

5. Exercise test abnormalities that do not meet any of the high risk criteria outlined in class $\mathrm{C}$ below

Clinical characteristics (must include all of the following):

1. NYHA classification of Class I or II

2. Exercise capacity $\leq 6$ METs

3. No evidence of congestive heart failure

4. No evidence of myocardial ischemia at rest or on the exercise test at or below 6 METs

5. Appropriate rise in systolic blood pressure during exercise

6. Absence of sustained or nonsustained ventricular tachycardia at rest or during exercise

7. Ability to satisfactorily self-monitor intensity of activity

Class C: Those at moderate-to-high risk for cardiovascular complications during exercise and/or unable to self-regulate activity or to understand recommended activity level

This classification includes individuals with any of the following diagnoses:

1. CAD

2. Mild or moderate valvular heart disease, excluding severe valvular stenosis or regurgitation

3. Congenital heart disease

4. Cardiomyopathy: ejection fraction $<30 \%$; includes stable patients with heart failure but not hypertrophic cardiomyopathy or recent myocarditis

5. Complex ventricular arrhythmias not well controlled

Clinical characteristics (any of the following):

1. NYHA classification of Class III or IV

2. Exercise stress test results

- Exercise capacity <6 METs

- Ischemic ST depression at a workload <6 METs

- Fall in systolic blood pressure below resting levels during exercise

- Nonsustained ventricular tachycardia during exercise

3. Previous episode of cardiac arrest with unknown cause (excluding cardiac arrest associated with myocardial infarction)

4. A medical problem that the physician believes may be life-threatening

\section{Class D: Unstable disease with activity restriction}

This classification includes individuals with any of the following:

1. Unstable angina

Activity guidelines: No activity is recommended unti conditions improve.

2. Severe and symptomatic valvular heart disease

3. Congenital heart disease

4. Heart failure that is not compensated

5. Uncontrolled arrhythmias

6. Other medical conditions that could be aggravated by exercise

ACLS, advanced cardiac life support; BLS, basic life support; CAD, coronary artery disease; NYHA, New York Heart Association; METs, metabolic equivalents.

Adapted from Fletcher GF, et al. Exercise standards for testing and training. Circulation 2001; 104: 1694-1740, ${ }^{99}$ with permission from Wolters Kluwer Health. 


\section{Table 13. Exercise Training Programs}

Exercise training programs include warm-up, resistance training, endurance training, and cool-down.

Examples:

Warm-up: stretching, warm-up exercise, low-intensity (slow) walking

Main exercises: aerobic exercise and resistance training at prescribed intensity

Cool-down: low-intensity (slow) walking, stretching, cooling-down exercise

[Aerobic exercise]

\begin{tabular}{|c|c|c|c|c|c|c|}
\hline \multirow[b]{2}{*}{ Intensity } & \multicolumn{3}{|c|}{ Intensity } & \multirow{2}{*}{$\begin{array}{l}\text { Duration of one } \\
\text { session (minutes) }\end{array}$} & \multicolumn{2}{|c|}{ Frequency } \\
\hline & $\%$ peak $\dot{\mathrm{VO}}_{2}$ & $\begin{array}{l}\text { Karvonen value } \\
\text { (k value) }\end{array}$ & $\begin{array}{c}\text { RPE } \\
\text { (Borg scale) }\end{array}$ & & Sessions/day & Days/week \\
\hline Low intensity & $20 \sim<40 \%$ & $0.3 \sim<0.4$ & $10 \sim<12$ & $5 \sim 10$ & $1 \sim 3$ & $3 \sim 5$ \\
\hline Moderate intensity & $40 \sim<60 \%$ & $0.4 \sim 0.6$ & $12 \sim 13$ & $15 \sim 30$ & $1 \sim 2$ & $3 \sim 5$ \\
\hline High intensity & $60 \sim 70 \%$ & $0.6 \sim 0.7$ & 13 & $20 \sim 60$ & $1 \sim 2$ & $3 \sim 7$ \\
\hline \multicolumn{7}{|c|}{ [Resistance training] } \\
\hline & \multicolumn{2}{|c|}{ Strength } & \multicolumn{4}{|c|}{ Frequency } \\
\hline Intensity & $\%$ of $1 R M$ & $\begin{array}{c}\text { RPE } \\
\text { (Borg scale) }\end{array}$ & $\begin{array}{l}\text { Repetitions } \\
\text { per set }\end{array}$ & $\begin{array}{l}\text { Sets per } \\
\text { exercise/day }\end{array}$ & \multicolumn{2}{|c|}{$\begin{array}{c}\text { Training sessions per week } \\
\text { (days/week) }\end{array}$} \\
\hline Low intensity & $20 \sim 30 \%$ & $10 \sim 11$ & $8 \sim 15$ & $1 \sim 3$ & \multicolumn{2}{|c|}{$2 \sim 3$} \\
\hline Moderate intensity & $40 \sim 60 \%$ & $11 \sim 13$ & $8 \sim 15$ & $1 \sim 3$ & \multicolumn{2}{|c|}{$2 \sim 3$} \\
\hline High intensity & $80 \%$ & $13 \sim 16$ & $8 \sim 15$ & 1 & \multicolumn{2}{|c|}{$2 \sim 3$} \\
\hline
\end{tabular}

Note: \% peak $\mathrm{VO}_{2}$ and \%1RM are calculated from actual measured value of individuals, and are not percentages of age-specific reference values.

peak $\dot{\mathrm{VO}}_{2}$, peak oxygen uptake; $1 \mathrm{RM}$, one repetition maximum; RPE, ratings of perceived exertion.

Source: American College of Sports Medicine. ACSM's Guidelines for Exercise Testing and Prescription, 7th edn, 2005,97 and Fletcher GF, et

al. Exercise standards for testing and training. Circulation 2001; 104: 1694-1740. ${ }^{99}$

\begin{tabular}{|ccc|}
\hline Table 14. & $\begin{array}{c}\text { Borg Scale for Ratings of Perceived Exertion } \\
\text { (RPE) }\end{array}$ & Exercise intensity (\%) \\
Scale & RPE & 100 \\
20 & Maximum exertion & 95 \\
19 & Very, very hard & \\
18 & & 85 \\
17 & Very hard & \\
16 & & 70 \\
15 & Hard & \\
14 & & \\
13 & Somewhat hard & 55 (corresponding to AT) \\
12 & & 40 \\
11 & Fairly light & \\
10 & & 20 \\
9 & Very light & \\
8 & & \\
7 & Very, very light & \\
6 & & \\
\hline
\end{tabular}

AT, anaerobic threshold.

Source: Borg GA. Perceived exertion. Exerc Sport Sci Rev 1974;

2: $131-153 .^{104}$ exercise capacity. Exercise intensity is classified into low, moderate and high categories. Tables $13^{97,99}$ and $14^{104}$ list the typical physical responses to, and the training loads for, low-, moderate- and high-intensity exercise training. Exercise training programs consist of warm-up, endurance training, resistance training, additional activities such as recreation, and cooldown. The reader should refer to the unabridged version of the guidelines for specific points and factors to be considered when developing exercise prescriptions. ${ }^{97,105}$

\section{General Guidelines for Exercise Training in the Patients With Cardiovascular Diseases}

General guidelines for exercise training in the patients with cardiovascular diseases include a) exercise only when feeling physically well; b) do not exercise vigorously soon after eating; c) adjust exercise to the weather; d) wear proper clothing and shoes; e) understand personal limitations; f) select appropriate exercises; and $\mathrm{g}$ ) be alert for symptoms. ${ }^{99}$

\section{Pathophysiology of Cardiovascular Diseases and Exercise Training}

\section{Myocardial Infarction}

\section{Acute Phase Rehabilitation}

\section{Class I}

1. Ambulation in the early phase of hospitalization ( $\geq 12$ hours after hospitalization) in patients with ST elevation myocardial infarction (STEMI) with neither recurrent ischemic chest pain, heart failure symptoms, nor severe arrhythmias. (Level of Evidence: C)

2. Supervised cardiac rehabilitation/secondary prevention programs in patients for whom exercise training is not contraindicated, especially intermediate- or high-risk patients or those with multiple coronary risk factors. (Level of Evidence: C) 
Table 15. Criteria for Evaluating the Results of an Exercise Stress Test Prior to Introducing Acute-Phase Rehabilitation in Patients With Acute Myocardial Infarction

Acute-phase rehabilitation can be introduced when the patient does not exhibit any of the following:

1. Symptoms such as chest pain, dyspnea, and palpitation

2. An increase in heart rate to $\geq 120 \mathrm{bpm}$ or by $\geq 40 \mathrm{bpm}$

3. The development of potentially dangerous cardiac arrhythmias

4. Ischemic ST depression of $\geq 1 \mathrm{~mm}$, or significant ST elevation

5. A change in systolic blood pressure by $\geq 20 \mathrm{mmHg}$ during the period before the patient was allowed to use a bedside commode

(The criteria for blood pressure are not used for patients 2 weeks after the onset of acute myocardial infarction.)

Patients who failed the exercise stress test should receive appropriate drug treatment or other measures, and undergo the same test on the next day.

\section{Class Ila}

1. To use a bedside commode after 12 24 hours of bed rest in patients with unstable hemodynamics or continued ischemia. (Level of Evidence: C)

\section{Class III}

1. Exercise stress test in the first 2 3 days after the onset of STEMI should avoid in patients with STEMI for whom reperfusion therapy has not been successful. (Level of Evidence: C)

2. Cardiac rehabilitation should avoid in patients with uncontrolled acute heart failure and those with persistent arrhythmias. (Level of Evidence: C)

\section{Recovery Phase Rehabilitation \\ Class I}

1. Cardiac rehabilitation/secondary prevention programs for patients recovering from STEMI. (Level of Evidence: B)

2. Exercise at anaerobic threshold (AT) level, 40 60\% of the peak $\dot{\mathrm{VO}}_{2}, 40 \sim 60 \%$ of maximum heart rate, or a Borg scale of 12 13. (Level of Evidence: A)

3. To perform 15 60 minute exercise sessions that are prescribed according to risk assessment by exercise at least 3 times a week ( 7 times whenever possible), and to increase their ADL. (Level of Evidence: B)

\section{Class Ila}

1. Submaximal exercise test on day 4 after onset or thereafter to predict prognosis, prescribe exercise training programs, and assess treatment efficacy. (Level of Evidence: B)

2. Symptom-limited exercise stress test on day 14 21 after onset to predict prognosis, prescribe exercise training programs, and assess treatment efficacy of cardiac rehabilitation. (Level of Evidence: B)

3. To promote long-term exercise adherence to increase the ADL and make exercise a daily habit. (Level of Evidence: A)

4. Exercise training for elderly patients in the same way as younger patients. (Level of Evidence: A)

5. Exercise training for clinically stable low-risk patients under proper instruction and supervision. (Level of Evidence: A)

6. Home-based exercise training programs with proper instruction and reporting. (Level of Evidence: A)

\section{Class Ila'}

1. Exercise training in patients with a large anterior wall infarction and poor cardiac function. (Level of Evidence: B)

2. Exercise training for patients $1 \sim 4$ weeks after placement of their stents. (Level of Evidence: B)
MI results in physical and mental deconditioning. Cardiac rehabilitation is provided to enhance recovery from physical/ mental deconditioning, reduce coronary risk factors, improve QOL, facilitate returning to work, and prevent recurrent MI or sudden death. The term cardiac rehabilitation refers to "coordinated, multifaceted interventions designed to optimize a cardiac patient's physical, psychological, and social functioning, in addition to stabilizing, slowing, or even reversing the progression of the underlying atherosclerotic processes, thereby reducing morbidity and mortality". ${ }^{78,106,107}$ Cardiac rehabilitation programs consist of (1) exercise training and prescription, (2) reduction of coronary risk factors and secondary prevention of cardiovascular disease, and (3) counseling on psychosocial factors and returning to work, and are classified by timing into the acute phase (phase I), early recovery phase (early phase II), late recovery phase (late phase II), and maintenance phase (phase III) programs (Table 1). ${ }^{108}$

\section{Effects of Exercise Training on Myocardial Infarction}

Cardiac rehabilitation has beneficial effects on physical function, and improves the outcomes that are most important to patients. It has been demonstrated that exercise training has pleiotropic effects such as improvement in coronary risk factors, prevention of atherosclerosis progression, ischemic events and thrombogenesis, antiinflammatory effect, and enhancement of endothelial function, skeletal muscle metabolism, and autonomic nervous system function in patients with CAD.

\section{Practice of Cardiac Rehabilitation in Patients With Myocardial Infarction}

Cardiac rehabilitation for patients with AMI is classified into three phases, and each phase has specific goals. In Japan, cardiac rehabilitation provided during hospitalization had been defined as acute-phase rehabilitation. However, in the current practice, phase I acute-phase rehabilitation is provided immediately after hospitalization, and is followed by phase II rehabilitation consisting with early recovery-phase rehabilitation during hospitalization and late recovery-phase rehabilitation after discharge. After participating in phase II programs, patients enter phase III maintenance phase rehabilitation.

\section{(1) Phase I (Acute Phase)}

Acute-phase cardiac rehabilitation during the first 1 or 2 week after the onset of MI is provided to ensure that patients are able to perform personal care safely such as meal preparation, bathing, and toilet use, and to start education on secondary prevention of cardiovascular events in the early phase of hospitalization. Patients who have no complications and can successfully complete an in-room walking test are transferred to the general ward and start early recovery-phase rehabilitation 


\begin{tabular}{|c|c|c|c|c|c|c|}
\hline Day & Goals & $\begin{array}{l}\text { Patient care/stress } \\
\text { test/rehabilitation }\end{array}$ & Activities & Meals & Excretion & Hygiene \\
\hline $\begin{array}{l}\text { Day } 1 \text { after } \\
\text { PCl }\end{array}$ & \multirow[t]{2}{*}{$\begin{array}{l}\text { - Prevent } \\
\text { complications of } \\
\text { AMI and } \\
\text { catheter-related } \\
\text { complications }\end{array}$} & $\begin{array}{l}\text { - Remove the } \\
\text { tourniquet, } \\
\text { disinfect wounds } \\
\text { - Allow use of a } \\
\text { bedside commode }\end{array}$ & $\begin{array}{l}\text { - After removing } \\
\text { the tourniquet, } \\
\text { allow the patient } \\
\text { to move freely on } \\
\text { the bed }\end{array}$ & \multirow{3}{*}{$\begin{array}{l}\text { - Regular diets for } \\
\text { patients with } \\
\text { cardiovascular } \\
\text { diseases } \\
\text { (1,600kcal/day, } \\
\text { salt } 6 \mathrm{~g} / \text { day }) \\
\text { - Set a water } \\
\text { consumption limit }\end{array}$} & \multirow[t]{2}{*}{$\begin{array}{l}\text { - Urine: Urinary } \\
\text { catheter } \\
\text { - Stool: Bedside } \\
\text { commode }\end{array}$} & $\begin{array}{l}\text { - Face washing: on the } \\
\text { bed } \\
\text { - Body cleaning: Bed- } \\
\text { bath. A caretaker } \\
\text { assists bed-bath of } \\
\text { the feet and back }\end{array}$ \\
\hline Day 2 & & $\begin{array}{l}\text { - Remove the } \\
\text { urinary catheter }\end{array}$ & $\begin{array}{l}\text { - Allow the patient } \\
\text { to move freely in } \\
\text { the room }\end{array}$ & & & \multirow{2}{*}{$\begin{array}{l}\text { - Face washing: Use } \\
\text { the washstand } \\
\text { - Body cleaning: } \\
\text { Bed-bath. A caretaker } \\
\text { assists bed-bath of } \\
\text { the feet and back }\end{array}$} \\
\hline Day 3 & $\begin{array}{l}\text { - Prevent } \\
\text { complications of } \\
\text { AMI }\end{array}$ & $\begin{array}{l}\text { - Remove the } \\
\text { venous access } \\
\text { device }\end{array}$ & $\begin{array}{l}\text { - Allow the patient } \\
\text { to walk to the } \\
\text { toilet }\end{array}$ & & \multirow[t]{12}{*}{$\begin{array}{l}\text { - Urine and } \\
\text { stool: Use the } \\
\text { toilet }\end{array}$} & \\
\hline Day 4 & $\begin{array}{l}\text { - No myocardial } \\
\text { ischemia }\end{array}$ & $\begin{array}{l}\text { - When patient can } \\
\text { pass a } 200 \mathrm{~m} \\
\text { walking test, } \\
\text { conduct } 200 \mathrm{~m} \\
\text { walking sessions } 3 \\
\text { times a day } \\
\text { - Ask a dietitian to } \\
\text { provide nutrition } \\
\text { education }\end{array}$ & $\begin{array}{l}\text { - Allow the patient } \\
\text { to walk around the } \\
\text { ward within a } \\
200 \mathrm{~m} \text { radius of } \\
\text { the room }\end{array}$ & $\begin{array}{l}\text { - Regular diets for } \\
\text { patients with } \\
\text { cardiovascular } \\
\text { diseases } \\
\text { (1,600kcal/day, } \\
\text { salt } 6 \mathrm{~g} / \mathrm{day}) \\
\text { - Allow drinking } \\
\text { water freely }\end{array}$ & & $\begin{array}{l}\text { - Face washing: Use } \\
\text { the washstand } \\
\text { - Bed-bath: A caretaker } \\
\text { assists bed-bath of } \\
\text { the back }\end{array}$ \\
\hline Day 5 & \multirow{3}{*}{$\begin{array}{l}\text { - No myocardial } \\
\text { ischemia } \\
\text { - The patient can } \\
\text { take his/her drugs } \\
\text { as prescribed } \\
\text { - The patient can } \\
\text { access information } \\
\text { about important } \\
\text { points for daily } \\
\text { living after } \\
\text { discharge }\end{array}$} & $\begin{array}{l}\text { - Order a cardiac } \\
\text { rehabilitation } \\
\text { program } \\
\text { - Confirm when the } \\
\text { cardiac rehabilita- } \\
\text { tion program begins } \\
\end{array}$ & & & & \\
\hline Day 6 & & $\begin{array}{l}\text { - Conduct a } \\
\text { submaximal } \\
\text { exercise test at }\end{array}$ & $\begin{array}{l}\text { - When patient } \\
\text { passes a } \\
\text { submaximal }\end{array}$ & & & $\begin{array}{l}\text { - Face washing: Use } \\
\text { the washstand } \\
\text { - Provide bed-bath }\end{array}$ \\
\hline Day 7 & & $\begin{array}{l}\text { entry in the cardiac } \\
\text { rehabilitation room } \\
\text { - If the patient has } \\
\text { not attended } \\
\text { the cardiac } \\
\text { rehabilitation, } \\
\text { conduct a } 500 \mathrm{~m} \\
\text { walking test }\end{array}$ & $\begin{array}{l}\text { stress test, allow } \\
\text { bathing, and } \\
\text { walking freely in } \\
\text { the hospital }\end{array}$ & & & $\begin{array}{l}\text { when requested by } \\
\text { the patient }\end{array}$ \\
\hline Day 8 & \multirow{3}{*}{$\begin{array}{l}\text { - No myocardial } \\
\text { ischemia } \\
\text { - The patient can } \\
\text { understand } \\
\text { important points } \\
\text { for daily living } \\
\text { after discharge } \\
\end{array}$} & \multirow{7}{*}{$\begin{array}{l}\text { - Conduct exercise } \\
\text { sessions at } \\
\text { the cardiac } \\
\text { rehabilitation room } \\
\text { (If the patient has } \\
\text { not attended the } \\
\text { program, conduct } \\
\text { a Master's single } \\
\text { test or allow } \\
\text { having a bath as } \\
\text { a trial) }\end{array}$} & & & & \multirow{7}{*}{$\begin{array}{l}\text { - Face washing: Use } \\
\text { the washstand } \\
\text { - Allow bathing when } \\
\text { the patient wants to } \\
\text { bathe }\end{array}$} \\
\hline Day 9 & & & & & & \\
\hline Day 10 & & & & & & \\
\hline Day 11 & \multirow{3}{*}{$\begin{array}{l}\text { - No ischemia at } \\
\text { submaximal } \\
\text { exercise } \\
\text { - The patient can } \\
\text { list important } \\
\text { points for daily } \\
\text { living after } \\
\text { discharge } \\
\end{array}$} & & & & & \\
\hline Day 12 & & & & & & \\
\hline Day 13 & & & & & & \\
\hline Day 14 & Discharge & & & & & \\
\hline
\end{tabular}

AMI, acute myocardial infarction.

programs. Table 15 lists criteria for the transition to the next phase. Table 16 shows the clinical pathway that is used for patients with AMI during the first 14 days of hospitalization in the National Cerebral and Cardiovascular Center (NCVC).

\section{(2) Early Phase II (Early Recovery Phase During Hospitalization)}

The recovery phase is defined as a period from 1 week to 3 months after the onset of STEMI. During the recovery phase, cardiac rehabilitation is provided to improve the range of physical activities and facilitate return to work and society with good mental and physical conditions by (1) conducting an exercise stress test to estimate prognostic risk, (2) providing proactive exercise training programs according to the individualized exercise prescription, (3) educating patients about secondary prevention and lifestyle intervention, and (4) providing comprehensive and systematic counseling on mental care and returning to work. 
Table 17. How to Determine Exercise Intensity During the Late Recovery Phase (Late Phase II) of Cardiac Rehabilitation for Patients With Acute Myocardial Infarction

A. $40 \sim 60 \%$ of $\mathrm{HR}$ reserve (=maximum HR-resting $\mathrm{HR}$ )

Karvonen formula: [maximum HR-resting HR] xk+resting HR

$\mathrm{k}=0.6$ for lower-risk patients (e.g., young patients with uncomplicated acute myocardial infarction), 0.4 0.5 for high-risk patients, and 0.3 0.5 for patients with heart failure

B. HR at AT level or $40 \sim 60 \%$ of peak $\dot{\mathrm{VO}}_{2}$

C. RPE: Borg scale of 12 13 (fairly light $\sim$ somewhat hard)

D. Simple setting: Resting HR+30 bpm (resting HR+20 bpm for patients receiving $\beta$-blockers)

Exercise intensity should be low in high-risk patients i.e., those with (1) left ventricular dysfunction (LVEF $<40 \%),(2)$ prolonged occlusion of the left anterior descending artery (patients who failed in reperfusion therapy), (3) patients with severe three-vessel disease, and (4) elderly patients ( $\geq 70$ years old).

AT, anaerobic threshold; HR, heart rate; LVEF, left ventricular ejection fraction; peak $\mathrm{VO}_{2}$, peak oxygen uptake; RPE, ratings of perceived exertion.

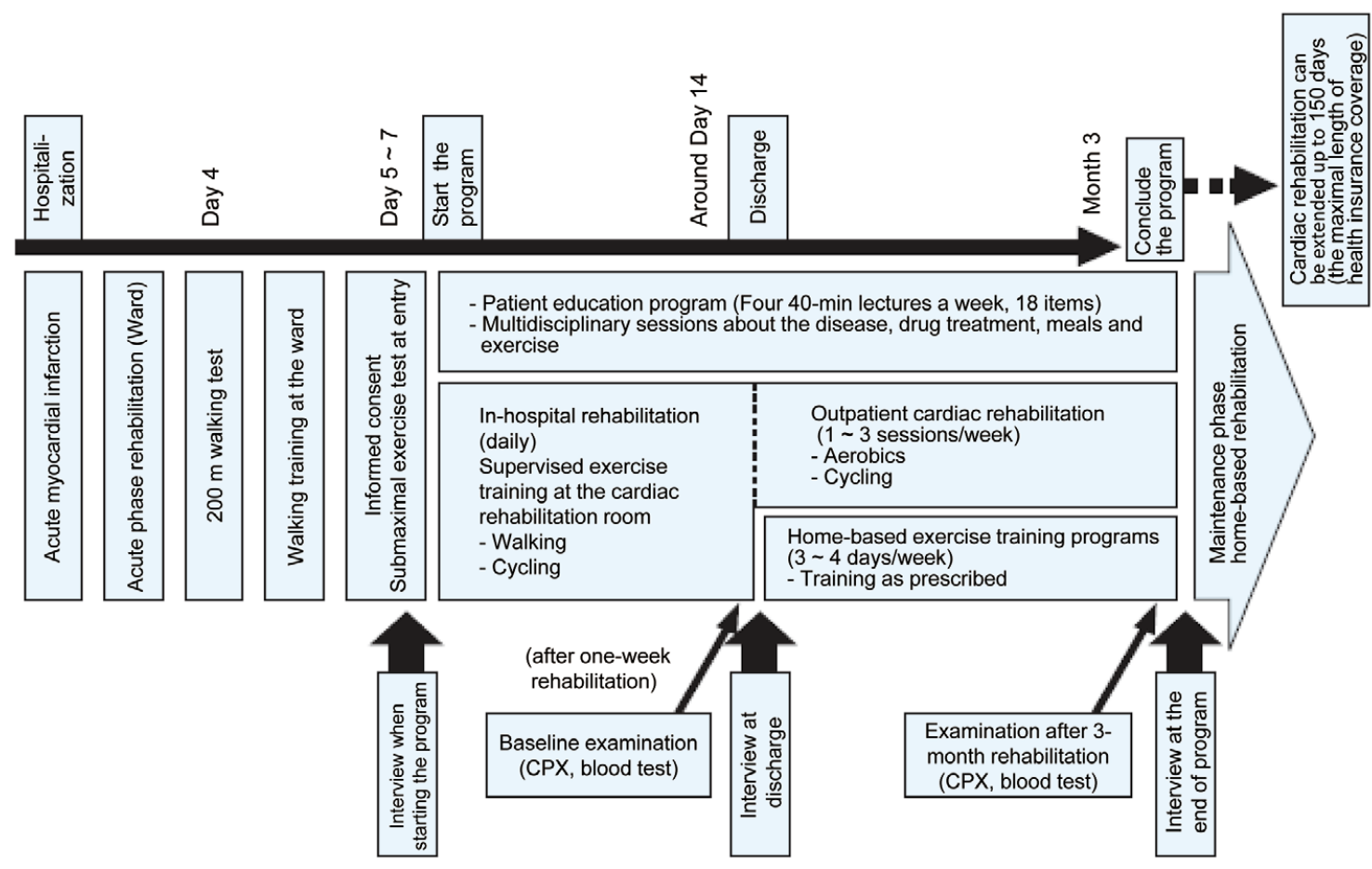

Figure 2. A cardiac rehabilitation program used in the National Cerebral and Cardiovascular Center for patients with acute myocardial infarction in the recovery phase. - On Day 4 of the 14-day clinical pathway (See Table 16), a $200 \mathrm{~m}$ walking test should be conducted, and patients who passed the test should attend a recovery-phase rehabilitation program at the cardiac rehabilitation room on Day 5 7 and thereafter. - After discharge, patients should attend outpatient supervised exercise training programs while continuing home-based exercise training programs. - Cardiopulmonary exercise test (CPX) and blood test should be performed one week and 3 months after the introducing of cardiac rehabilitation to assess exercise capacity and coronary risk factors and prescribe exercise training programs.

The widespread use of reperfusion therapy has substantially decreased the duration of hospitalization in patients with AMI, and patients do not have sufficient time to undergo recoveryphase cardiac rehabilitation during hospitalization. Because phase II cardiac rehabilitation is critical to prevent deterioration of QOL and prognosis, patients are recommended to enter recovery-phase cardiac rehabilitation programs during hospitalization, and continue outpatient recovery-phase (late phase II) cardiac rehabilitation programs after discharge.

\section{(3) Late Phase II (Late Recovery Phase, Outpatient Programs)}

After discharge for AMI, patients usually visit the clinic every other week for follow-up. At each visit, patients should participate in comprehensive programs including smoking secession, dietary education and lifestyle intervention. Under the appropriate risk management, individualized exercise programs are designed to achieve recovery of ADL to the levels they were before the onset of MI (Table 17). Exercise stress test 

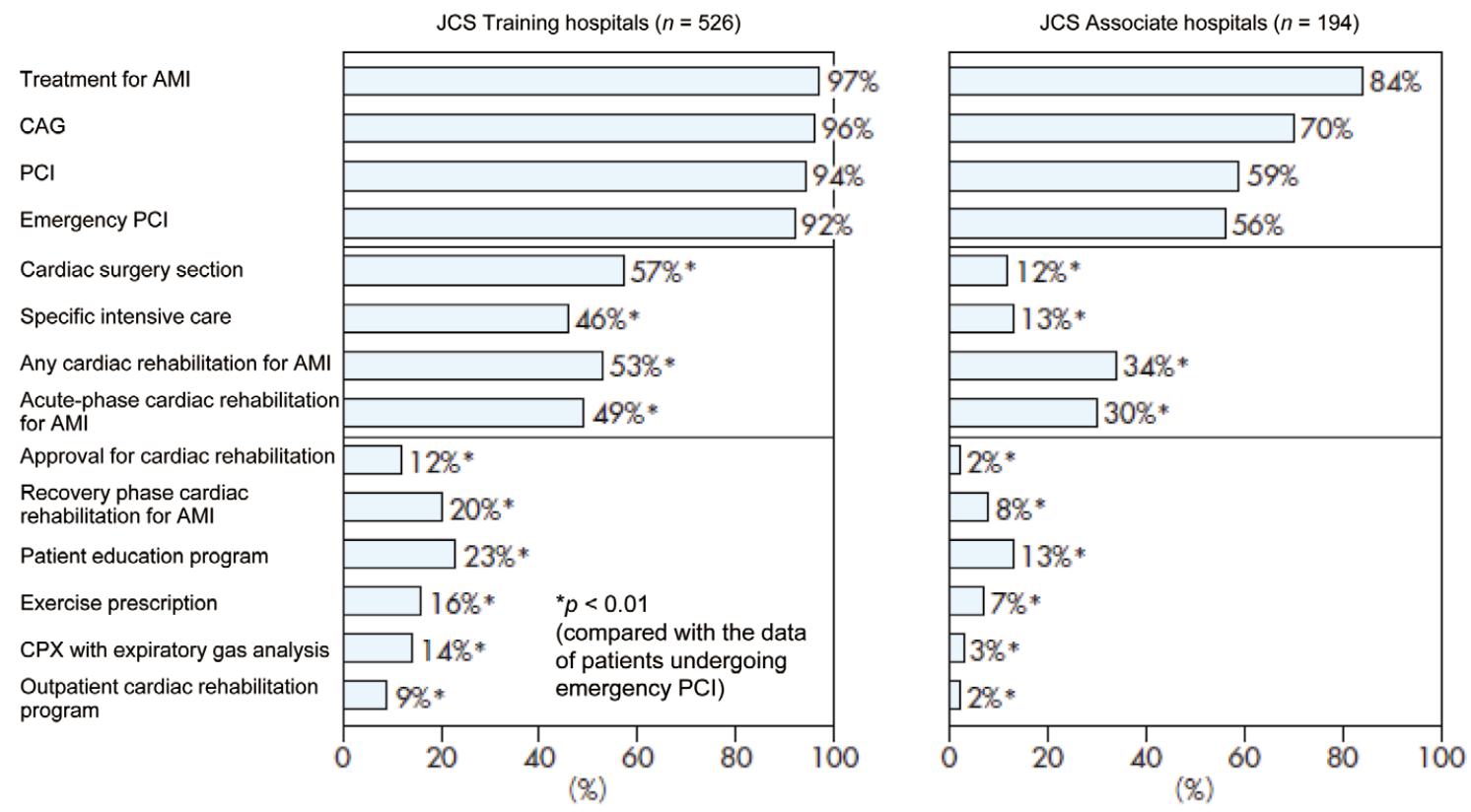

Figure 3. Nationwide survey on the treatment of acute myocardial infarction in cardiovascular hospitals in Japan. AMI, acute myocardial infarction; CAG, coronary angiography; CPX, cardiopulmonary exercise test; JCS, Japanese Circulation Society; MHLW, Ministry of Health, Labor and Welfare; PCI, percutaneous coronary intervention. Adapted from Goto Y, et al. Poor implementation of cardiac rehabilitation despite broad dissemination of coronary interventions for acute myocardial infarction in Japan. Circ J 2007; 71: 173-179. ${ }^{115}$

should be performed 1,3 , and 6 (or 5 ) months after onset or at the end of the phase II rehabilitation to reissue exercise prescription, evaluate the efficacy of exercise training, and assess the prognosis of the patients. The NHI System in Japan reimburses for most parts of cardiac rehabilitation programs for 150 days. After this period, patients should enter maintenancephase cardiac rehabilitation.

\section{(4) Phase III (Maintenance Phase, Outpatient Programs)}

Maintenance-phase cardiac rehabilitation is provided to prevent recurrent cardiovascular events, and should be continued for life. Cardiac rehabilitation should be established as part of the patients' ADL.

\section{Clinical Pathways for Myocardial Infarction}

Patients with AMI are treated according to clinical pathways including acute-phase cardiac rehabilitation programs. ${ }^{109} \mathrm{Be}-$ cause serious complications of AMI develop most frequently during the first week after onset, the NCVC is using the 14day clinical pathway shown in Table $\mathbf{1 6}$ to care for patients who have been successfully treated with reperfusion therapy and have Killip I type AMI without complications with a peak creatine kinase (CK) level of $\geq 1,500 \mathrm{U} / \mathrm{L}$, while a 10 -day clinical pathway is used to manage patients with small infarctions with peak CK levels of $<1,500 \mathrm{U} / \mathrm{L}$. Figure 2 illustrates a rehabilitation program of NCVC for AMI patients from the acute to the chronic stage of the disease. Before encouraging patients to increase their ADL, patients undergo exercise stress test to monitor changes in symptoms, heart rate, blood pressure, and ECG during exercise. Table 15 shows the criteria for exercise stress test from the NCVC. On day 6 of onset and thereafter, patients whom exercise training is not contraindicated should enter recovery-phase cardiac rehabilitation programs.

\section{Local Collaborative Clinical Pathways Including Cardiac Rehabilitation for Acute Myocardial Infarction}

Many of the medical institutions where cardiac rehabilitation is not available are small- or medium-sized hospitals. The most common reasons for unavailability of a rehabilitation program are a lack of staff members, exercise area, or suitable equipment. ${ }^{110}$ It is highly likely that such small- or medium-sized hospitals cannot satisfy the current MHLW requirements for providing cardiovascular rehabilitation or cannot treat a sufficiently large number of patients requiring cardiac rehabilitation. However, these medical institutions may establish "local clinical pathways including cardiac rehabilitation for AMI" by collaborating with local institutions that provide outpatient cardiac rehabilitation programs. ${ }^{111}$

\section{Patient Education}

Because the duration of hospitalization has become shorter, and patients with AMI cannot receive sufficient education during their stay regarding secondary prevention, healthcare professionals should provide the minimum necessary information during the acute phase, and continue patient education during recovery-phase cardiac rehabilitation programs. During the acute-phase cardiac rehabilitation, healthcare professionals should (1) instruct the patient how to manage chest pain and provide contact information for when unmanageable chest pain occurs; (2) instruct the patient how to use nitroglycerin sublingual tablets or sprays; (3) encourage the patient and his/ 
her family members to learn how to perform cardiopulmonary resuscitation; (4) explain the patient's coronary risk factors; (5) motivate the patient to participate in cardiac rehabilitation and improve lifestyle; and (6) encourage the patient to quit smoking and maintain a tobacco-free lifestyle. ${ }^{112}$ Patient education during the acute-phase cardiac rehabilitation should be focused on instructing the patient on how to deal with emergency situations and encouraging secondary prevention activities.

\section{Points for Further Consideration}

The widespread use of percutaneous coronary intervention (PCI) in patients with AMI has decreased the duration of hospitalization and allowed early return to society. Exercise training, which exerts multifaceted effects on physical and mental health, should be included as a standard part of the treatment of AMI. ${ }^{59}$

Although there is discussion on the effect of $\beta$-blocker therapy on the response to exercise training after MI, mean changes in exercise capacity were not significantly different between post-MI patients receiving and not receiving $\beta$-blockers during cardiac rehabilitation program. ${ }^{113}$ Also, after AMI, the effects of exercise training and $\beta$-blockers on heart rate variability are not redundant. ${ }^{114}$

Although the benefits of exercise training in post-MI patients have been demonstrated, the use of cardiac rehabilitation is quite limited, which contrasts with the widespread use of PCI in the treatment of MI. In a survey in 2009 in Japan, ${ }^{110}$ only $21 \%$ of post-MI patients participated in outpatient cardiac rehabilitation programs after discharge (Figure 3). ${ }^{115}$ The number of institutions providing outpatient cardiac rehabilitation programs must be increased substantially to establish cardiac rehabilitation as an essential step for post-MI patients, for whom in-hospital treatment of AMI has become shorter than ever. ${ }^{116}$

\section{Patients After Cardiac Surgery}

\section{Class I}

1. Exercise training is recommended for patients after $\mathrm{CABG}$ because it is effective in improving symptoms and exercise capacity, and in controlling coronary risk factors. (Level of

\section{Evidence: A)}

2. Exercise training is recommended for patients after valve surgery, and is prescribed to improve symptoms and exercise capacity. (Level of Evidence: A)

\section{Class Ila}

1. It is appropriate to encourage patients to start ambulation as soon as possible after cardiac surgery. (Level of Evidence: B)

2. Patients should be carefully observed for occurrence of dysphagia after cardiac surgery. (Level of Evidence: B)

3. Unnecessary use of chest straps or excessive restriction of body movement that limits chest wall motion may delay the recovery of exercise capacity and increase the occurrence of complications. (Level of Evidence: C)

4. If not contraindicated, it is appropriate to conduct exercise training in all patients after cardiac surgery to improve exercise capacity and QOL as well as to decrease the incidence of cardiac events. The use of exercise training for patients with cardiac dysfunction or musculoskeletal disorder should be considered individually according to the pathophysiological conditions. (Level of Evidence: B)

\section{Class Illb}

1. The use of an incentive spirometer should be considered to prevent the occurrence of respiratory complications after cardiac surgery. (Level of Evidence: B)

\section{Phase I (Acute Phase)}

\section{(1) Goals of Acute Phase Rehabilitation}

Excessive bed rest after cardiac surgery may cause physical deconditioning and induce various complications. It is thus important during acute-phase rehabilitation after cardiac surgery that patients should be treated to stabilize hemodynamics and are encouraged to begin ambulation and return to their previous level of physical activity without delay.

\section{(2) Rehabilitation Schedules (Tables 18,19,20)}

Recently, the advancement of minimally invasive surgery and postsurgical management has made it possible to satisfy the criteria for postoperative ambulation, and thereby patients may leave the bed earlier and progress through cardiac rehabilita-

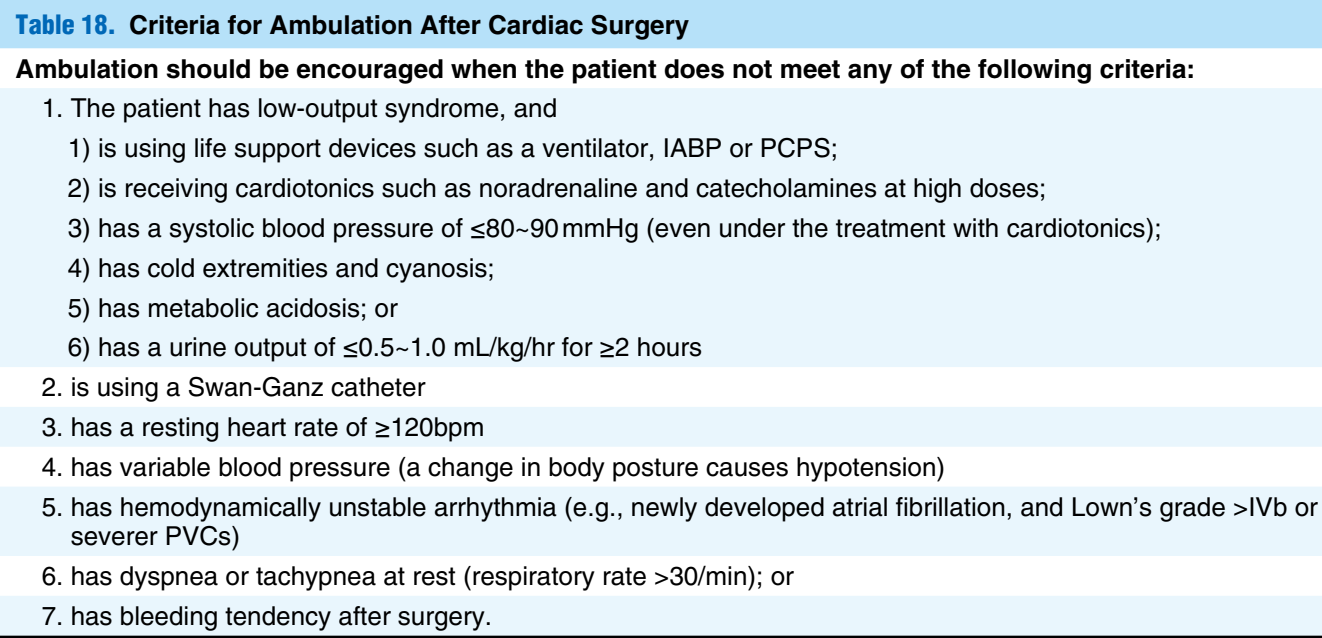

IABP, intra-aortic balloon pumping; PCPS, percutaneous cardiopulmonary support; PVCs, premature ventricular contractions. 


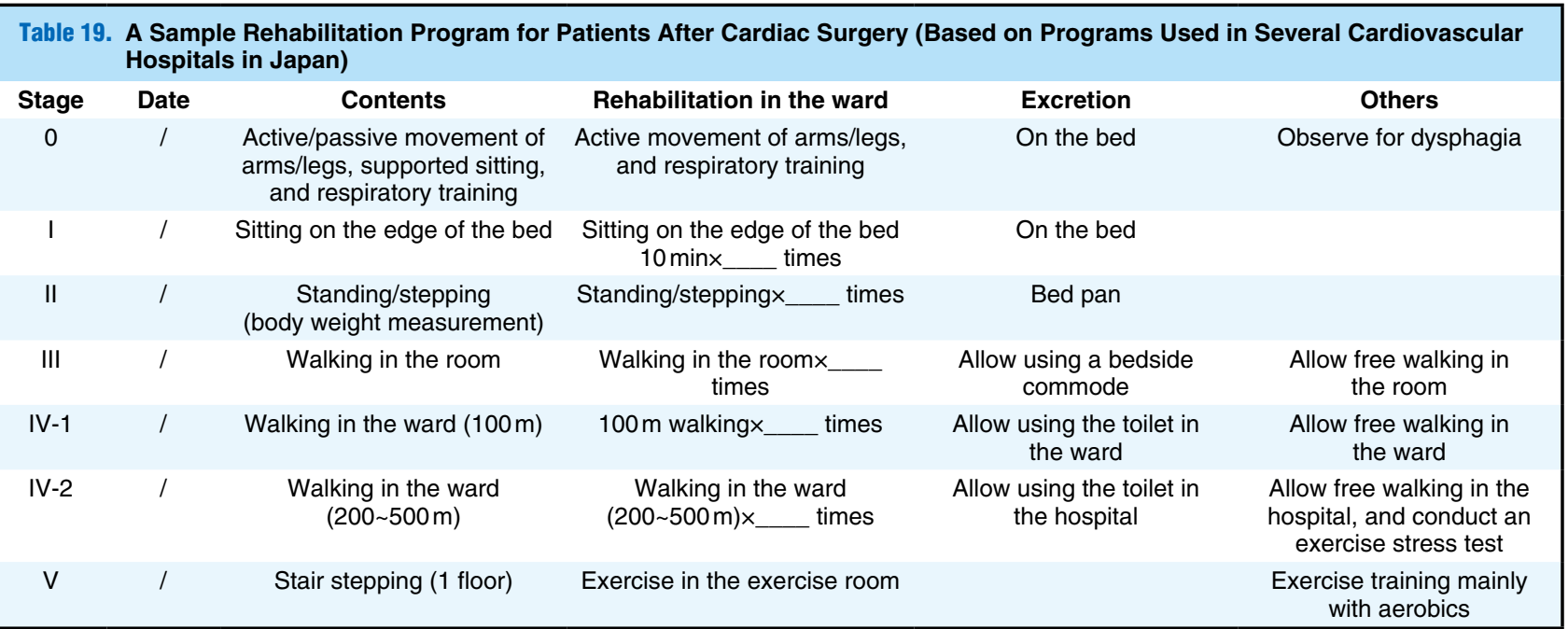

Table 20. Criteria for Evaluating the Results of Exercise Stress Tests (Criteria for Moving to the Next Level
of Exercise Training)
Exercise training does not induce
1. Chest pain, severe shortness of breath, severe fatigue (Borg scale of $>13$ ), dizziness, lightheadedness,
or leg pain;
2. Cyanosis, facial pallor, or cold sweat (objectively);
3. Tachypnea ( $>30 /$ min);
4. Atrial fibrillation or an increase in the incidence of arrhythmias;
5. Ischemic ECG changes;
6. Excessive changes in blood pressure;
7. An increase in heart rate by $\geq 30 b p m ;$ or
8. A decrease in oxygen saturation to $\leq 90 \%$.

tion faster. ${ }^{117}$

In cardiac rehabilitation after cardiac surgery, the increment of load is larger than that seen after AMI. It is considered fine to prolong walking distance or exercise intensity on the day of surgery as possible provided that satisfy the criteria. Recently, the wide-spread use of minimally invasive surgery and the advancement of cardioplegia and postoperative management have enabled fast-track rehabilitation programs in which the patient is weaned from the ventilator on the day of surgery, and starts standing and walking on postoperative day 1 with a goal of progressing to ambulation in the ward by postoperative day $4 \sim 5$.

\section{(3) Dysphagia and Respiratory Complications}

\section{1) Dysphagia}

Because dysphagia may cause aspiration pneumonia, the screening, evaluation, and treatment of dysphagia after cardiac surgery are important. It has been reported that dysphagia develops in 3 51\% of patients undergoing cardiac surgery, ${ }^{118,119}$ and was associated with risk factors such as the presence of prolonged postoperative heart failure and the use of ventilators for 48 hours or longer.

\section{2) Respiratory Physiotherapy}

After open heart surgery, the movement of chest wall is restricted, both physically and mentally due to postoperative pain from sternotomy, and respiratory function declines. Although incentive spirometers have been used after cardiac surgery to prevent respiratory complications, negative results have been reported. ${ }^{120-122}$

\section{3) Median Sternotomy and Use of Chest Straps}

In countries other than Japan, "chest straps" are not used for patients after cardiac surgery. Unnecessary use of chest straps or excessive restriction of body movement that limits chest wall motion may delay the recovery of exercise capacity and increase the occurrence of complications. ${ }^{123}$

\section{Phase II (Recovery Phase) and Later Phases}

\section{(1) Effects of Exercise Training}

In addition to the benefits described in Chapter II-1 "Physical Effects", exercise training after open heart surgery may increase graft patency. Cardiac rehabilitation also decreases rehospitalization rate and associated healthcare costs after open heart surgery.

\section{(2) Methods of Exercise Training}

The patient in the acute phase after open heart surgery should undergo an exercise stress test (cardiopulmonary exercise test [CPX] whenever possible) on postoperative day 4 10 when he/she is able to walk for $30 \sim 100 \mathrm{~m}$, and has started aerobic exercise using a cycle ergometer or treadmill. It should be confirmed that the patient (1) is afebrile and shows an improvement in inflammatory response, (2) dose not have a large 
volume of pericardial or pleural effusion, (3) has no newly developed atrial fibrillation/flutter, and (4) has a hemoglobin level of $\geq 8 \mathrm{~g} / \mathrm{dL}$ with a tendency towards improvement.

Exercise at an AT level is preferable. When CPX is not feasible, the target heart rate during exercise training may be calculated using the Karvonen formula and the patient's actual maximum heart rate with a $\mathrm{k}$ coefficient of $0.3 \sim 0.6$. However, heart rate response is often blunted immediately after cardiac surgery, and the target heart rate must be selected carefully.

Although resistance training is effective, patients should avoid strenuous upper extremity exercises during the first 3 months after open heart surgery. Patients may start range of motion (ROM) exercise early after cardiac surgery. ${ }^{124}$

\section{(3) Outpatient Rehabilitation Programs}

Because cardiac rehabilitation should be continued for life, exercise training after discharge is important. Since the duration of hospitalization after cardiac surgery has become increasingly shorter, healthcare professionals should assess the patient's lifestyle in the early stage of hospitalization and instruct him or her on how to continue exercise training after discharge to ensure sufficient patient education by the time of discharge. In Japan, because many patients are discharged home after cardiac surgery rather than to a cardiac rehabilitation center, healthcare professionals should instruct patients and their family members on how to continue exercise training and disease management in the home. It has been reported that multi-faceted, comprehensive programs improved the 10-year, event-free survival. ${ }^{125}$

\section{Characteristics of Women Undergoing CABG and the Results of Cardiac Rehabilitation}

Women undergoing CABG are generally older than similar male patients, often have concomitant hypertension and/or diabetes with advanced cardiovascular disease, and have a small body surface area with small coronary vessels. Due to the infrequent use of internal thoracic artery grafting, the operative mortality rate and 30-day mortality rate are higher in women than men undergoing CABG. ${ }^{126-134}$ Off-pump CABG has been increasingly used in Japan, and the results of female patients are particularly favorable. However, few studies have investigated gender difference in the effects of cardiac rehabilitation after cardiac surgery. ${ }^{135}$

\section{Intervention for Angina Pectoris/Coronary Artery Diseases}

Class I

1. Cardiac rehabilitation for the purpose of improving prognosis is recommended for patients with CAD. (Level of Evidence: A)

\section{Class Ila}

1. Exercise training or cardiac rehabilitation is appropriate to improve anginal symptoms. (Level of Evidence: B)

2. Cardiac rehabilitation is appropriate to prevent the progression of coronary lesions and improve myocardial perfusion. (Level of Evidence: B)

3. Exercise training is appropriate for patients after PCI because it is beneficial in preventing restenosis and cardiovascular events. (Level of Evidence: B)

\section{Class Ila'}

1. It is appropriate to perform an exercise stress test and begin exercise training 1 3 days after PCI. (Level of Evidence: B)

Although the scientific evidence is weak (Level of Evidence: B), it has been reported that exercise training improves anginal symptoms, prevents the progression of coronary lesions, and increases myocardial perfusion in patients with CAD. ${ }^{89,136}$

Although cardiac rehabilitation is theoretically indicated for patients after PCI, ${ }^{137,138}$ there is no Class I evidence supporting the benefits of exercise training in this patient population. ${ }^{106,139}$ Because the number of patients undergoing PCI is increasing, and patients have a risk of subacute stent thrombosis in the early period after stenting, it is important to establish criteria for early implementation of comprehensive programs centering on exercise training. In Japan, reports on the effect of exercise training in patients after PCI have been published, and Class IIa' evidence is becoming available (Level of Evidence: B). ${ }^{140-143}$ Although no large-scale randomized studies have been conducted, no safety concerns have been reported.

\section{Exercise Training}

Exercise training for patients with angina pectoris should be implemented according to Chapter III "General Rules of Exercise Prescriptions". It is desirable that exercise training be prescribed according to the results of CPX (Table 13). The

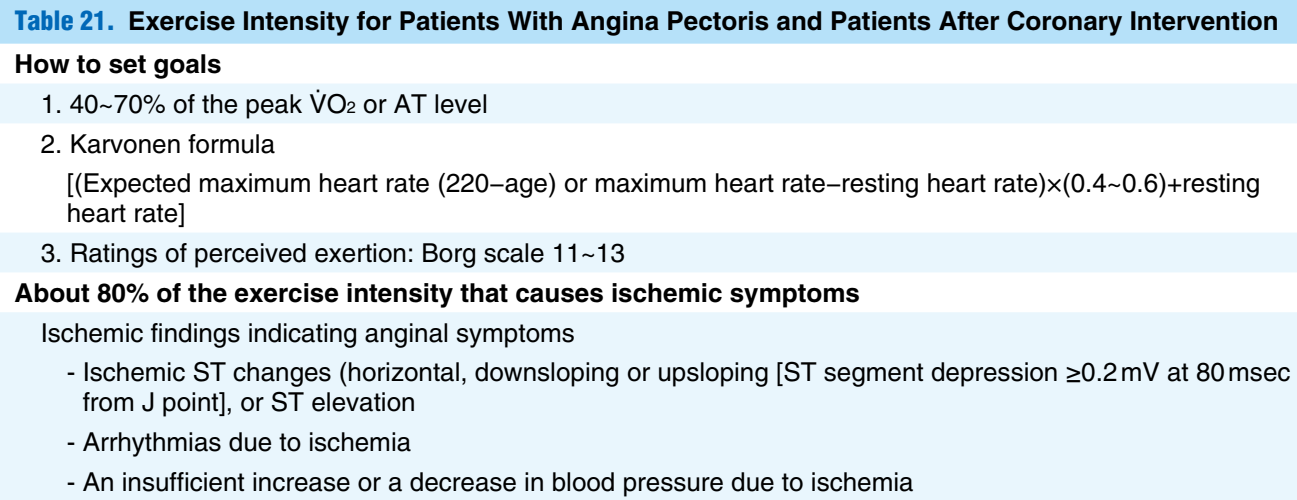

AT, anaerobic threshold; peak $\dot{\mathrm{V}}_{2}$, peak oxygen uptake. 
intensity and frequency of exercise training should be prescribed carefully to ensure the safety of exercise training. Because patients may have mild myocardial ischemia after PCI that does not cause symptoms and ischemic ST changes, physicians should carefully evaluate the severity of residual coronary lesions of each patient. Patents with stents should drink water during training sessions and take their medication as they are at risk of thrombosis not only immediately after but also for several years after receiving their stents. ${ }^{144}$

There are no established criteria for the exercise prescription for patients with angina pectoris. However, considering the fact that patients undergoing elective stenting return to their normal ADLs after treatment, patients may be able to start exercise training with a maximum workload of $6 \sim 7$ metabolic equivalents (METs) immediately after receiving their stents (Table 21).

\section{Instruction and Management of Outpatient Exercise Training Sessions}

Outpatient exercise training for patients after PCI should be performed according to the secondary prevention programs for patients after MI (Section IV-1 "Myocardial Infarction"). Secondary prevention in patients after PCI should include appropriate types and doses of drugs that achieve the goals described in the relevant guidelines. ${ }^{145}$ In Japan, elderly women with CAD, a situation that is becoming increasingly common, generally respond well to PCI but often have more coronary risk factors than do their male counterparts. Women should therefore be followed up carefully after PCI.

\section{Arrhythmias}

Some types of arrhythmias are induced by exercise, while other types of arrhythmias are prevented by exercise. Physicians should consider both types of arrhythmias when discussing the relationship between arrhythmias and exercise. However, few RCTs have fully described this relationship.

\section{Class I}

1. Exercise training programs which can prevent sudden death in patients with MI are recommended, but only when these patients do not meet the criteria for terminating exercise training. (Level of Evidence: A)

\section{Class Ila'}

1. Physicians should consider prescribing exercise training for patients with premature ventricular contraction (PVC) who do not meet the criteria for terminating exercise training because exercise training is beneficial for QOL in patients with atrial fibrillation, those with pacemakers, and those with implantable cardioverter defibrillators (ICDs). (Level of Evidence: C)

\section{Class III}

1. Patients with ventricular arrhythmia who meet criteria for terminating exercise training should avoid exercise training.

\section{Exercise and Arrhythmias ${ }^{146}$}

Arrhythmias may be induced by a combination of various factors including emotional stress, anxiety, smoking, drinking, taking caffeine, ingestion of diuretics or digitalis, or insomnia.

\section{Effects of Exercise Training on Arrhythmias}

\section{(1) Ventricular Arrhythmias (Table 22)}

It is believed that exercise training decreases the incidence of ventricular arrhythmias via the mechanisms listed in Table 22.

\section{(2) Atrial Fibrillation}

Atrial fibrillation impairs exercise capacity. ${ }^{147}$ The mechanisms of the impaired exercise capacity are thought to be decreased cardiac output by diminished left ventricular filling pressure caused by loss of atrial contraction, tachycardia, or irregular rhythms, or reduced vasodilation responses to nitric oxide (NO) during exercise. ${ }^{148}$ Palpitations and heart failure symptoms caused by atrial fibrillation can deteriorate QOL.

Exercise training improves the impaired exercise capacity and deterioration of QOL in patients with atrial fibrillation. Although currently available evidence is limited, exercise training is expected to be effective in patients with chronic atrial fibrillation, and is highly recommended for this patient population. ${ }^{149-152}$

Although currently available evidence is also limited regarding the effect of exercise training in patients with atrial fibrillation after cardiac surgery, patients undergoing cardiac surgery should begin cardiac rehabilitation programs before surgery and restart the programs immediately after surgery to prevent the occurrence of atrial fibrillation after cardiac surgery. ${ }^{153}$

\section{(3) Pacemaker}

Exercise capacity is impaired in patients who depend on pacemakers, but it has been reported that exercise capacity improves after exercise training with optimal pacing rate settings. ${ }^{154,155}$ To achieve the goal of cardiac rehabilitation, i.e., obtaining a significant improvement in exercise capacity, ${ }^{156}$ physicians should not only prescribe appropriate exercise training programs but also adjust the pacing rate appropriately on the basis of exercise stress test. The reader should refer to Chapter IV5-3-1 "Patients with ICD or CRT-D" for exercise training in patients with cardiac resynchronization therapy (CRT) or ICDs.

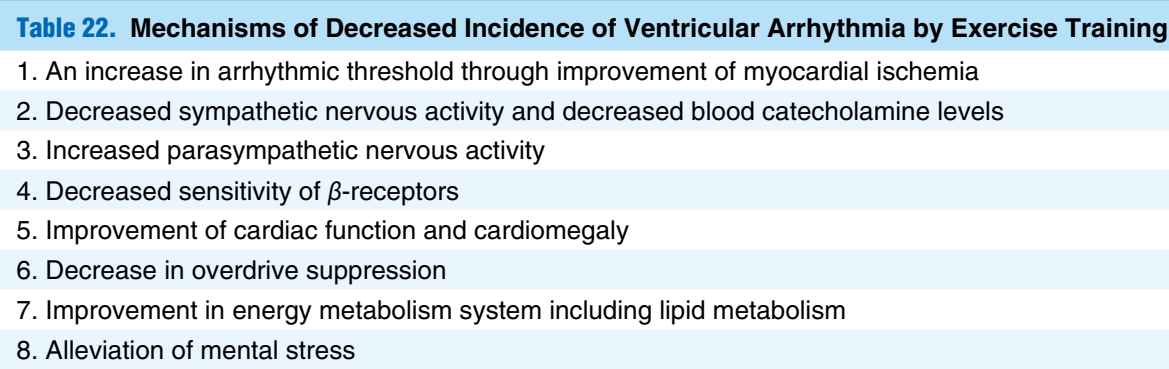




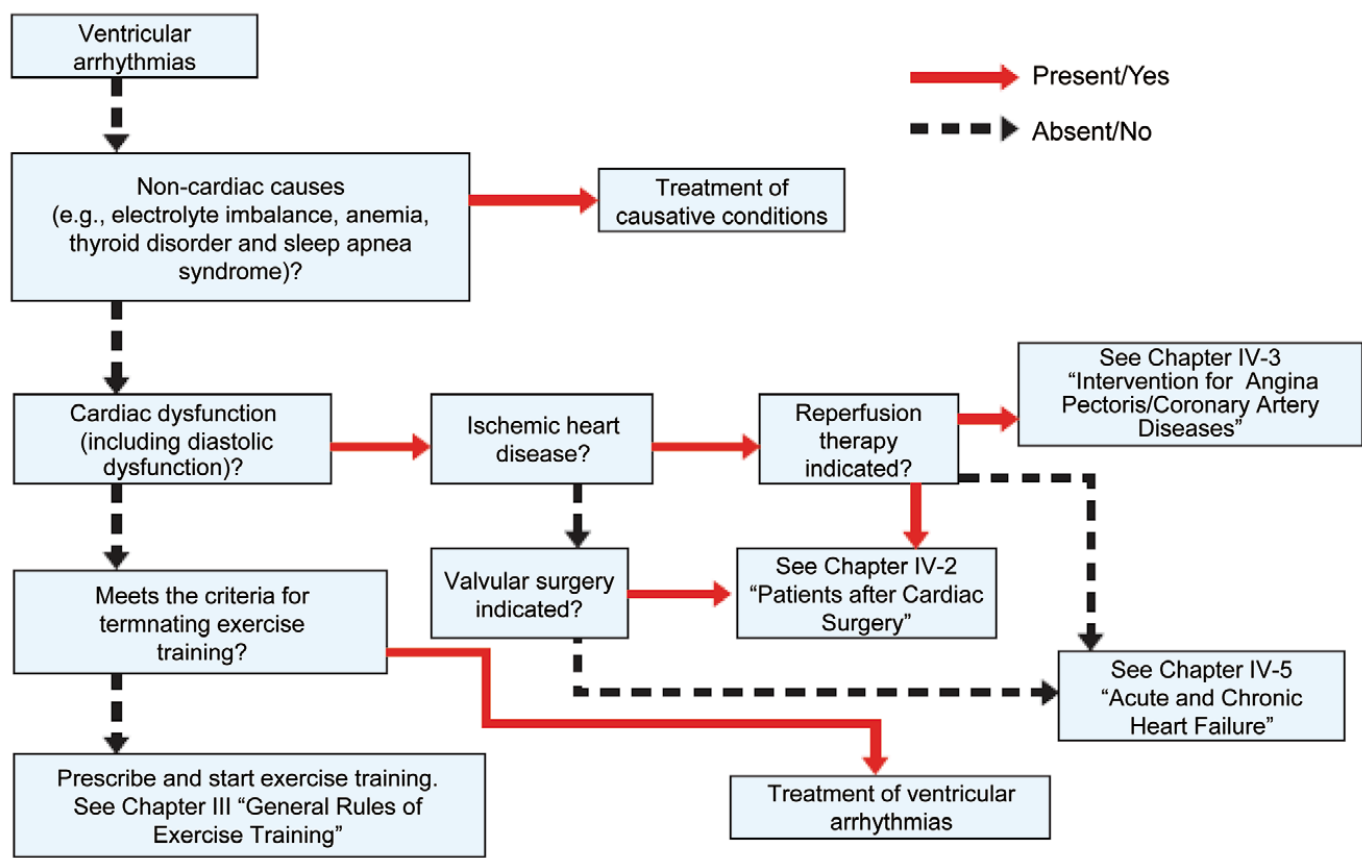

Figure 4. Flowchart for introducing of exercise training in patients with ventricular arrhythmias.

\section{Practice of Exercise Training in Patients With} Arrhythmias

(1) Ventricular Arrhythmias

\section{1) Exercise Stress Test}

Figure 4 shows a flowchart of how to introduce exercise training. Before beginning exercise training, patients must undergo an exercise stress test to assess their exercise capacity and the severity of ventricular arrhythmias. When ventricular arrhythmias meets the criteria for terminating exercise training before the patient has achieved the AT level during the exercise stress test, the patient should receive appropriate treatments (i.e., drug treatment, ablation, and/or treatment of causes of arrhythmias) to control the ventricular arrhythmias prior to introducing exercise training.

\section{2) Exercise Prescription}

Patients who have no cardiac dysfunction and have not shown exacerbation of arrhythmia during exercise stress test should begin training at a moderate intensity (refer to Chapter IV-5 "Acute and Chronic Heart Failure" for exercise training in patients with cardiac dysfunction).

\section{3) After Introducing of Exercise Training Programs}

When arrhythmia that meets the criteria for terminating exercise training after the introducing of exercise training program, physicians should consider whether the episode was caused by the high intensity of exercise training or represents an exacerbation of arrhythmia. When arrhythmia meets the criteria for terminating exercise training even after shortening the duration of each exercise session or decreasing the intensity of exercise, physicians should identify the cause of exacerbation and treat the arrhythmia.

\section{(2) Atrial Fibrillation (Figure 5)}

\section{1) Exercise Stress Test}

Because of no evidence for optimal heart rates at rest and during exercise, patients should be individually assessed to determine whether their heart rates are adequately controlled. Because only a small number of Japanese patients with atrial fibrillation have achieved the goal of adequate rate control as recommended in the guidelines for the management of patients with atrial fibrillation published by the American College of Cardiology Foundation (ACCF)/AHA before the introducing of exercise training, physicians should consider an exercise stress test for patients in whom heart failure is controlled (see Chapter IV-5 "Acute and Chronic Heart Failure") and heart rate at rest is less than $110 \mathrm{bpm}$. The feasibility of exercise training should be determined on the basis of the change in heart rate, symptoms, duration of exercise, and peak METs during exercise stress test, among other findings. Physicians may use the heart rate variability index $[\mathrm{HRVI}=\{$ (maximum heart rate)-(resting heart rate) $\} /$ (duration of exercise)] to define adequate rate control as a HRVI of $\leq 10 \mathrm{bpm} / \mathrm{min}$.

\section{2) Exercise Prescription}

It is difficult to use heart rate as a determinant of exercise intensity for patients with atrial fibrillation. On a CPX, exercise intensity for patients with atrial fibrillation should be determined by calculating the walking speed using the intensity or METs at the AT level. On a treadmill test is conducted, the target exercise intensity should be determined at 40 60\% and $20 \sim 40 \%$ of the METs at the maximum workload for patients who need moderate and light intensity exercise programs, respectively. When patients cannot undergo an exercise stress test, physicians should prescribe exercise intensity using Borg scale for ratings of perceived exertion. ${ }^{104}$ Patients without car- 


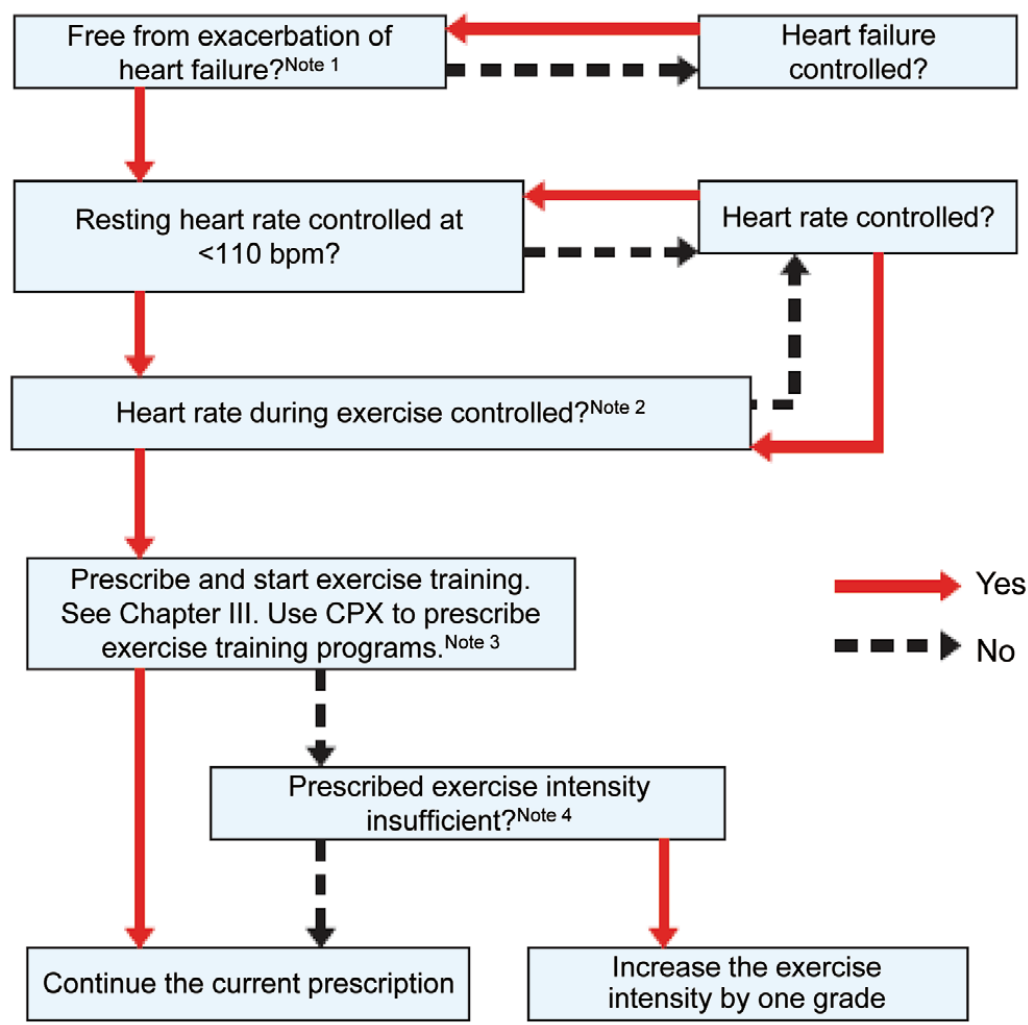

Figure 5. Flowchart for exercise training in patients with atrial fibrillation. AT, anaerobic threshold; CPX, cardiopulmonary exercise test; HRVI, heart rate variability index; METs, metabolic equivalents; $\mathrm{SpO}_{2}$, percutaneous arterial oxygen saturation. Note 1: Symptoms of heart failure (e.g., dyspnea, edema and anorexia) and signs of heart failure (an increase in body weight by $\geq 2 \mathrm{~kg}$ within one week, decreases in $\mathrm{SpO}_{2}$ at rest and immediately after exercise as compared with baseline, and pulmonary congestion and worsening of pleural effusion on X-ray). Note 2: The feasibility of exercise training should be determined on the basis of the change in pulse rate, symptoms, duration of exercise, and peak METs during exercise stress test, among other findings. Physicians may define adequate rate control as a HRVI of $\leq 10 \mathrm{bpm} / \mathrm{min}$. Note 3 : When a CPX is conducted to determine target exercise intensity, it should be determined by calculating the recommended walking speed using the intensity at the AT or the METs during exercise. The target exercise intensity should be determined at 40 60\% and $20 \sim 40 \%$ of the METs of exercise at the maximum workload for patients who need moderate and light intensity exercise programs, respectively, during treadmill tests. Note 4: In some patients, oxygen intake and pulse rate at the AT are higher than those in the maximum workload. In such patients, physicians should consider intensifying the exercise when the prescribed exercise is considered insufficient on the basis of blood pressure, pulse rate and symptoms after introducing exercise training programs.

diac dysfunction should begin exercise at a moderate intensity. In some patients with atrial fibrillation, oxygen uptake and pulse rate at the AT level are higher than $40 \sim 60 \%$ of those the maximum workload, and moderate intensity exercise may not be sufficient to reach the AT level. In such patients, physicians should consider intensifying the exercise when the prescribed exercise is considered insufficient on the basis of blood pressure, pulse rate, and symptoms after introducing exercise training programs.

\section{3) After Introducing of Exercise Training Programs}

Patients with a resting heart rate of $>110 \mathrm{bpm}$ should not attend the session for the day, or consider switching to less intense or shorter session. When signs and symptoms of heart failure (e.g., dyspnea, edema, and anorexia for symptoms, and increased body weight $\geq 2 \mathrm{~kg}$ within one week, decreases in $\mathrm{SpO}_{2}$ [percutaneous arterial oxygen saturation] at rest and immediately after exercise as compared with baseline, pulmonary congestion and worsening of pleural effusion on X-ray for signs) develop after the introducing of exercise training, exercise intensity should be decreased and treatment of heart rhythm disorder and heart failure should be given.

\section{(3) Pacemakers (Figures 6 and 7)}

\section{1) Exercise Stress Test}

Exercise stress test must be performed in patients with pacemakers before introducing exercise training programs not only to assess exercise capacity and determine exercise intensity but also to evaluate the heart rate response of the pacemaker and the appropriateness of pacemaker setting.

\section{2) Exercise Prescription}

Exercise intensity for patients with sick sinus syndrome who require pacemakers may be calculated on the basis of the results of a treadmill test or CPX when the pacemaker uses physiological sensors to control pacing rate. This should be calculated using METs, the target heart rate calculated using the Karvonen formula, or the Borg scale on a treadmill test 


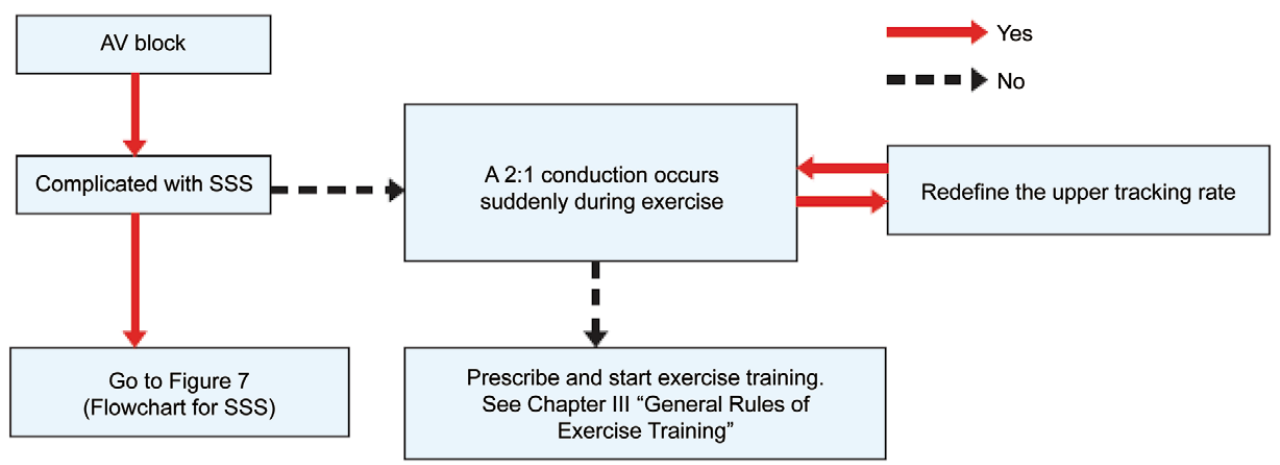

Figure 6. Points to check during exercise stress test in patients with pacemakers. AV block, atrioventricular block; SSS, sick sinus syndrome.

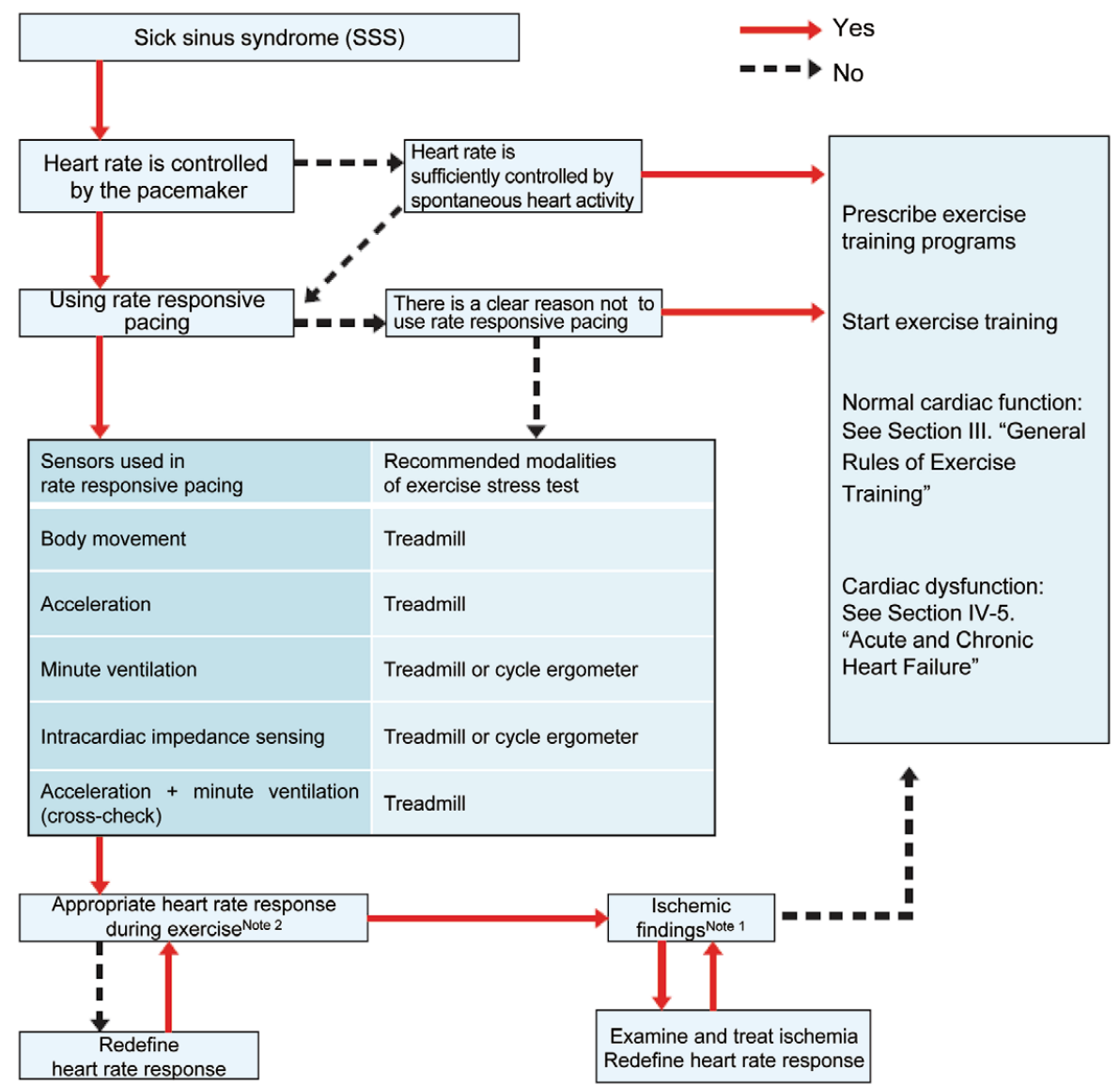

Figure 7. Flowchart for exercise training in patients with SSS. $\mathrm{VO}_{2}$, oxygen uptake. Note 1: Ischemic findings including chest pain and other symptoms, and levelling-off in $\mathrm{VO}_{2}$. Note 2: Example of maximum heart rate during exercise: for patients with normal cardiac function, (220-age) $\times 86 \%$; for patients with cardiac dysfunction, $(220-$ age $) \times 75 \%$.

when the pacemaker uses nonphysiological sensors. Exercise intensity for patients without cardiac dysfunction should be moderate. Because an excessively high target heart rate may exacerbate heart failure or cause myocardial ischemia, physicians should monitor patients carefully. 


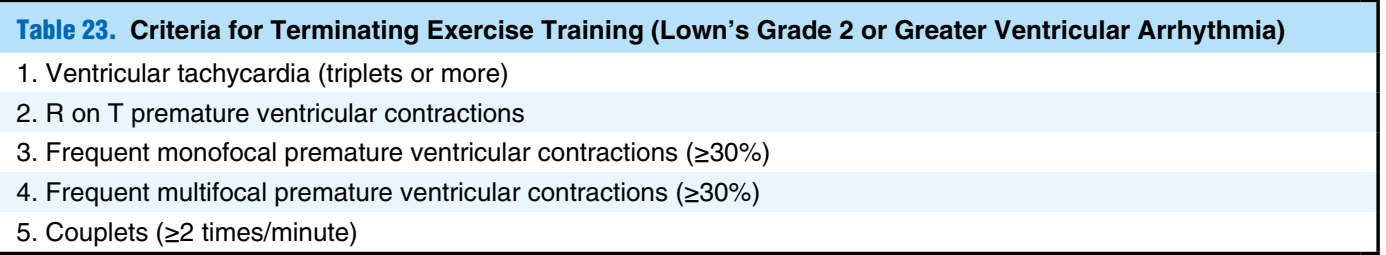

Source: American College of Sports Medicine. ACSM's Guidelines for Exercise Testing and Prescription, 6th edn Philadelphia: Lippincott Williams \& Wilkins; 2000. ${ }^{157}$

\begin{abstract}
3) After Introducing of Exercise Training Programs During each exercise session, the heart rate response should be assessed using an ECG monitor. The patient should be carefully observed for whether an increase in heart rate triggers the development of myocardial ischemia or an exacerbation of heart failure.
\end{abstract}

\section{Criteria for Terminating Exercise Training}

The American College of Sports Medicine (ACSM) recommends that exercise training should be terminated in patients with Lown's grade 2 or greater ventricular arrhythmia (Table 23). ${ }^{157}$

The criteria for terminating exercise training may differ whether exercise training is performed supervised or non-supervised. Physicians should also consider the recurrence of ventricular arrhythmias and the occurrence of specific types of PVC that may lead to sudden death.

\section{Cardiac Events During Exercise}

Mead et al. have reported that ventricular fibrillation during exercise training developed at an average of one per 6,000 person-hours of supervised exercise training. ${ }^{158}$ Haskel et al. have reported that among 748,133 person-hours of exercise training conducted in 22 institutions, 10 cases of fatal cardiac events and 37 cases of non-fatal cardiac events developed during training sessions. ${ }^{159}$ These findings indicated that the incidence of cardiac events during exercise training is low.

In Japan, there have been no cases of cardiac events caused by arrhythmias during exercise training in a study in which a total of 88,373 person-hours of supervised exercise training sessions were analyzed. Exercise training is considered safe. ${ }^{160}$

In a survey conducted in an inpatient cardiac rehabilitation center in Italy to evaluate the incidence of cardiac arrhythmias during exercise in patients after CABG surgery, cardiac arrhythmias were $33.5 \%$ and were more frequent in patients with hypertension, diabetes, and dyslipidemia, compared with the rate in patients free from these diseases. The incidence of arrhythmias was significantly higher both in patients older than 70 years as compared with the rate in younger patients, and in patients who discontinued amiodarone as compared with those who did not interrupt the drug treatment. ${ }^{161}$ In a retrospective survey in Japan, the incidence of postoperative arrhythmia clearly related to exercise training was $0.92 \%$ in 1,293 patients after cardiac surgery. ${ }^{162}$

\section{Summary}

There have been only a limited number of studies providing evidence on the effect of exercise training on arrhythmia and the risk of arrhythmia during exercise training. Patients who may develop arrhythmia during exercise should perform exercise sessions under ECG monitoring, and should conform to the criteria for terminating exercise training. However, exercise training is expected to be effective in the treatment of ventricular arrhythmias, and further studies of the effect of exercise training on arrhythmias should be conducted.

\section{Acute and Chronic Heart Failure}

\section{Cardiac Rehabilitation in Patients With Acute Heart Failure \\ Class I}

1. An educational program on the prevention of heart failure recurrence and the enhancement of self-management is recommended to implement for all patients with acute heart failure. (Level of Evidence: C)

\section{Class Ila}

1. It is appropriate that all patients with acute heart failure participate in cardiac rehabilitation programs when the patients' condition are stabilized. (Level of Evidence: C)

\section{(1) Significance of Cardiac Rehabilitation in Patients With Acute Heart Failure}

Cardiac rehabilitation in patients with acute heart failure is conducted to 1) prevent adverse effects of excessive bed rest (e.g., physical/mental deconditioning, bedsores, and pulmonary embolism) by promoting early ambulation; 2) design, share, and implement plans to ensure prompt and safe discharge and returning to work; 3) improve the QOL of patients by improving their exercise capacity; and 4) prevent recurrent heart failure and rehospitalization through patient education and disease management. It is also important to motivate patients to participate in and continue cardiac rehabilitation programs after discharge.

\section{(2) Physical Therapy and Exercise Training for Patients With Acute Heart Failure}

Low-intensity physical therapy and exercise training are feasible for patients with acute heart failure who are hemodynamically stable and asymptomatic at rest even when they are receiving intravenous infusion. Patients are recommended to perform bending exercise of limbs, low-intensity resistance training on the bed, and standing position practice and tiptoeing on the bedside floor.

\section{(3) Mental Support and Counseling for Patients With Acute Heart Failure}

Patients with acute heart failure are severely anxious and mentally unstable. Mental support is thus important during the acute phase of acute heart failure to relieve mental pain and improve QOL during hospitalization. Table 24 lists methods for providing mental support. Healthcare professionals should carefully monitor patients for anxiety and depressive state, provide mental counseling promptly when such symptoms occur, and consider drug treatment and cognitive behavioral therapy when- 
Table 24. Mental Support for Patients With Acute-Phase Heart Failure

1. Secure sufficient time to meet family members from an early phase of treatment.

2. Listen to the patient.

3. Explain the purpose and methods of examinations and treatments in advance to reassure the patient.

4. Talk to the patient to facilitate his or her expression of worries and questions.

5. Ensure that the patient has a sufficient length of sleep.

6. Encourage the patient to get refreshed to prevent excessive stress due to limited activities and visits.

7. Explain the schedule of examinations, treatment, and rehabilitation to provide the patient with information for future planning.

8. Suspect restlessness or CCU psychosis syndrome when the patient is uneasiness or insomnia, and consider preventive measures.

$\mathrm{CCU}$, coronary care unit.

\section{Table 25. Effects of Exercise Training in Patients With Heart Failure}

1. Exercise capacity: improvement

2. Effects on the heart

a) Left ventricular function: No change or slight improvement in LVEF at rest, increase in the increment of cardiac output during exercise, and improvement in left ventricular early diastolic function

b) Coronary circulation: Improvement in coronary endothelial function, improvement in myocardial perfusion during exercise, and increase in coronary collateral flow

c) Left ventricular remodeling: Prevention (or inhibition) of progression, decrease in BNP

3. Peripheral effects

a) Skeletal muscle: Increase in muscle mass/strength, improvement in aerobic metabolism, and increase in expression of antioxidant enzymes

b) Respiratory muscles: Improvement in respiratory muscle function

c) Vascular endothelium: Improvement in endothelium-dependent vasodilation responses, and increase in expression of eNOS

4. Neurohumoral factors

a) Autonomic nervous system function: Inhibition of sympathetic nervous activity, increase in parasympathetic activation, and improvement in heart rate variability

b) Ventilatory response: Improvement in ventilatory response and $\mathrm{CO}_{2}$ sensitivity of the respiratory center

c) Inflammatory reactions: Decreases in inflammatory cytokines (TNF- $a$ ) and CRP

5. QOL: Improvement in health-related QOL

6. Long-term prognosis: Decrease in hospitalizations due to heart failure, increase in event-free survival, and decrease in total mortality (meta-analyses)

BNP, brain natriuretic peptide; CRP, C-reactive protein; eNOS, endothelial nitric oxide synthase; LVEF, left ventricular ejection fraction; TNF, tumor necrosis factor; QOL, quality of life.

ever necessary.

\section{(4) Patient Education for Patients With Acute Heart Failure}

Patient education on how to manage heart failure and prevent rehospitalization should be performed from the early phase of hospitalization. Patients should be instructed to (1) measure, record, and manage body weight; (2) become aware of recurrence of heart failure; (3) maintain compliance to drug treatment; (4) limit salt intake; (5) limit alcohol intake and quit smoking; (6) continue appropriate exercise training. It is desirable that patients begin cardiac rehabilitation programs after stabilization of heart failure symptoms during hospitalization, and should shift to outpatient cardiac rehabilitation programs after discharge (see Chapter IV-5-2-4 "Cardiac Rehabilitation and Disease Management Programs in Chronic Heart Failure").

\section{Cardiac Rehabilitation in Chronic Heart Failure}

Class I

1. It is recommended that chronic heart failure patients with impaired exercise capacity undergo exercise training aiming to improve symptoms and exercise capacity. (Level of Evidence: A)

\section{Class Ila}

1. Exercise training aiming to improve exercise capacity and QOL and to decrease cardiac events is an appropriate intervention for all chronic heart failure patients with reduced systolic function. (Level of Evidence: B)

2. Exercise training aiming to improve exercise capacity is an appropriate intervention for diastolic heart failure patients with impaired exercise capacity. (Level of Evidence: B)

3. Exercise training including low-intensity resistance training that is aiming to improve exercise capacity is an appropriate intervention for chronic heart failure patients with reduced muscle strength. (Level of Evidence: C)

\section{(1) Effects of Exercise Training in Patients With Chronic Heart Failure}

By the 1970s, bed rest had been recommended for patients with heart failure. Since the 1990s, researchers have reported that exercise training for patients with stable chronic heart failure is beneficial in improving exercise capacity,, ,21,30,163-171 QOL ${ }^{4,13,163,169,172}$ and long-term prognosis, ${ }^{4,173}$ and so on (Table 25). In the HF-ACTION study in which 2,331 patients with stable chronic heart failure randomly received either drug treatment alone or drug treatment plus exercise training, since adherence to exercise training was low, exercise capacity, 
Table 26. Contraindications for Exercise Training in Patients With Heart Failure
I. Absolute contraindications
1. Exacerbation of heart failure symptoms (e.g., dyspnea, easy fatigability) during the last week
2. Unstable angina or low-threshold myocardial ischemia that is induced by slow walking on a flat surface (2 METs)
3. Severe valvular heart disease indicated for surgery, especially aortic stenosis
4. Severe left ventricular outflow tract obstruction (hypertrophic obstructive cardiomyopathy)
5. Untreated severe exercise-induced arrhythmia (ventricular fibrillation, sustained ventricular tachycardia)
6. Active myocarditis
7. Acute systemic disease or fever
8. Other diseases in which exercise is contraindicated (moderate or severe aortic aneurysm, severe hypertension, thrombophlebitis, embolism that developed in the last 2 weeks, and serious organ diseases)
II. Relative contraindications
1. NYHA classification of Class IV heart failure or heart failure requiring intravenous cardiotonics
2. Heart failure with an increase in body weight by $\geq 2 \mathrm{~kg}$ during the last week
3. Exercise-induced decrease in systolic blood pressure
4. Moderate left ventricular outflow tract obstruction
5. Exercise-induced moderate arrhythmia (e.g., nonsustained ventricular tachycardia, tachycardiac atrial fibrillation)
6. Advanced atrioventricular block
7. Exacerbation of exercise-induced symptoms (e.g., fatigue, dizziness, excessive sweating, dyspnea)
III. Not contraindicated
1. Elderly patients
2. Decreased LVEF
3. Heart failure during the use of LVADs
4. Use of ICDs

ICDs, implantable cardioverter defibrillators; LVADs, left ventricular assist devices; LVEF, left ventricular ejection fraction; METs, metabolic equivalents; NYHA, New York Heart Association.

QOL and long-term prognosis did not improve as expected. ${ }^{174}$ A meta-analysis in 2010 indicated that exercise training does not decrease total mortality or total hospital admission in patients with chronic heart failure, but does decrease hospitalization due to heart failure and improves HRQOL. ${ }^{172}$

Exercise training is effective in patients with chronic heart failure regardless of sex, ${ }^{175}$ the type of underlying disease (ischemic or non-ischemic disease), ${ }^{7,176}$ use of $\beta$-blockers, ${ }^{177,178}$ left ventricular function, ${ }^{179,180}$ New York Heart Association (NYHA) classification (Class II or III). ${ }^{174,181,182}$ It also have been indicated in Japan that exercise training improves exercise capacity in Japanese patients with diastolic heart failure or severe cardiac dysfunction that is characterized by an LVEF of $<25 \%$. ${ }^{182}$ It has been reported that exercise training helps improve exercise capacity, relieve anxiety and depression, and improve QOL in patients with ICDs or cardiac resynchronization therapy defibrillators (CRT-Ds).

\section{(2) Indications, Contraindications, and Safety of Exercise Training in Patients With Chronic Heart Failure}

\section{1) Indications}

All patients with chronic heart failure must be assessed carefully to determine whether exercise training is indicated. ${ }^{176}$ Exercise training is indicated for patients with stable controlled chronic heart failure with a NYHA classification of Class II or III. Chronic heart failure is considered "stable" when the patient has not experienced symptoms (e.g., dyspnea, easy fatigability) and signs of heart failure (e.g., edema, pulmonary congestion) during the last week, and is considered "controlled" when the patient is euvolemic and does not have moderate or severe leg edema or pulmonary congestion. ${ }^{183}$

\section{2) Contraindications}

Table 26 lists absolute and relative contraindications of exercise training in chronic heart failure. While systemic exercise training is not indicated for patients with NYHA classification of Class IV heart failure, some patients may be indicated for partial skeletal muscle training. ${ }^{184}$ Although exercise training is generally contraindicated for patients who are elderly, have reduced LVEF, are using ventricular assist devices, or are using ICDs, these patients may be indicated for exercise training.

\section{3) Safety}

In a systematic review of studies of exercise training in patients with heart failure, there were no reports of deaths that were directly related to exercise during more than 60,000 patient-hours of exercise training. ${ }^{173}$ The risk of cardiac events during exercise training is no higher than that seen in other cardiac rehabilitation programs in patients with heart failure. Cardiac events that may develop during exercise training include hypotension, arrhythmias, and exacerbation of heart failure. ${ }^{163,184}$ The incidence of cardiac events requiring hospitalization is lower in patients undergoing exercise training than in those not undergoing it.4,37,173,174 In a study of 111 patients with moderate or severe heart failure who participated in a 3-month exercise training program in Japan, the incidence of cardiac events (exacerbation of heart failure, hypotension, or arrhythmia) that necessitated termination and interruption of exercise training was $5 \%$ and $8 \%$, respectively. ${ }^{184}$

\section{(3) Practice of Cardiac Rehabilitation and Exercise Training in Patients With Heart Failure}

1) Basic Points of Cardiac Rehabilitation Programs for Patients With Heart Failure

Because the purpose of cardiac rehabilitation and exercise 
Table 27. Exercise Prescriptions for Patients With Heart Failure

Type of exercise

- Walking (begin with supervised indoor walking), cycle ergometer, light aerobics, low-intensity resistance training.

- Jogging, swimming, and vigorous aerobics are not recommended for patients with heart failure.

\section{Exercise intensity}

[Early phase]

- Exercise should start with indoor walking at $50 \sim 80 \mathrm{~m} / \mathrm{min}$ for $5 \sim 10 \mathrm{~min}$ or a cycle ergometer at 10 20W for 5 10 $\mathrm{min}$.

- The duration and intensity of exercise should be increased gradually over 1 month by observing signs/symptoms during exercise.

- Resting HR+30 bpm (resting HR+20 bpm for patients receiving $\beta$-blockers) may be set as a simple HR target.

[Goal in the stable phase]

a) The target $\mathrm{HR}$ is set at $40 \sim 60 \%$ of the peak $\mathrm{VO}_{2}$ or at AT level.

b) The target HR is set at $30 \sim 50 \%$ of $\mathrm{HR}$ reserve, or $50 \sim 70 \%$ of the maximum $\mathrm{HR}$.

- The target $\mathrm{HR}$ is calculated using the Karvonen formula [(maximum HR-resting HR) $\times \mathrm{k}+$ resting HR], and is set at $\mathrm{k}=0.4 \sim 0.5$ for mild heart failure (NYHA classification of Class I II) and k=0.3 0.4 for moderate or severe heart failure (NYHA classification of Class III).

c) Target RPE is set at Borg scale 11 13 (fairly light $\sim$ somewhat hard).

\section{Duration}

- Training should begin with 5 10 min/session, 2 sessions a day, and increase gradually to $30 \sim 60 \mathrm{~min} /$ day $(20 \sim 30 \mathrm{~min} / \mathrm{session}, 2$ sessions a day).

\section{Frequency}

- 3 5 days a week (The frequency may be increased to 3 days/week for patients with severe heart failure, and 5 days/week for those with mild heart failure.)

- Low-intensity resistance training may be added at a frequency of 2 3 days/week.

Precautions

- Exercise during the first month should be light in intensity, and physicians should watch the patient for exacerbations of heart failure.

- Programs should begin with supervised training, and should be continued with combination of supervised and non-supervised (homebased) exercise training programs during the stable phase.

- Changes in symptoms, body weight, and blood BNP levels should be observed carefully.

$\mathrm{AT}$, anaerobic threshold; BNP, brain natriuretic peptide; $\mathrm{HR}$, heart rate; peak $\dot{\mathrm{VO}}_{2}$, peak oxygen uptake; RPE, ratings of perceived exertion.

\section{Table 28. Findings Suggestive of Excessive Exercise Intensity}

1. Symptoms (continued malaise, fatigue that lasts into the following day, and increase in Borg scale by $\geq 2$ in the same exercise)

2. Tendency toward increased body weight (by $\geq 2 \mathrm{~kg}$ in one week)

3. Tendency toward increased heart rate (by $\geq 10 \mathrm{bpm}$ at rest or during the same exercise)

4. Tendency toward increased BNP level (by $\geq 100 \mathrm{pg} / \mathrm{mL}$ as compared with the previous measurement)

BNP, brain natriuretic peptide.

Adopted from Goto Y. Cardiac rehabilitation as heart failure therapy. Journal of the Japanese Association of Cardiac Rehabilitation (JJCR)

2008; 13: 273-277,187 with modification.

training programs for patients with heart failure includes improving exercise capacity, improving QOL, preventing rehospitalization, and achieving a better long-term prognosis, programs must consist of (1) exercise training, (2) patient education, and (3) counseling. As patients with heart failure may vary in cause and severity, exercise training programs should be prescribed individually by physicians on the basis of clinical findings of the results of exercise stress test, and should be carefully implemented. Training programs should begin with supervised exercise training under ECG monitoring. ${ }^{99,176,185}$ After the safety of the prescribed program is confirmed, patients should begin non-supervised home-based exercise training programs.

2) Exercise Prescriptions for Patients With Heart Failure Table 27 lists exercise prescriptions currently recommended for patients with heart failure.

Rhythmic aerobic exercises are commonly recommended. Patients with reduced muscle strength are expected to improve exercise capacity when they perform both rhythmic aerobic exercises and low-intensity resistance training. When prescribing exercise intensity, physicians should consider not only current symptoms and exercise capacity data but also variable parameters of severity of heart failure and patients' characteristics such as left ventricular function, change of BNP levels, and drug treatment. Patients with a BNP level of $\geq 400 \mathrm{pg} / \mathrm{mL}$ should begin exercise training at an extremely low intensity, and should be carefully followed up for changes in their heart failure condition. ${ }^{184,186}$

\section{3) Considerations During Exercise Training in Heart Failure: Monitoring and Revising Exercise Prescriptions}

To ensure the safety and efficacy of exercise training in heart failure, monitoring of patients and periodic revising of their exercise prescriptions are essential.

Table 28 lists findings that indicate exercise intensity is excessive during training. ${ }^{187}$ 
Although transient exacerbation of heart failure such as body weight gain and exacerbated congestion may develop 1 2 weeks after the introducing of exercise training, these events may be treated by limiting water intake, temporally increasing the dose of diuretics, and temporally reducing the amount of exercise. ${ }^{183}$ Patients who completed one-month training programs may shift to home-based (non-supervised) exercise training programs. However, it is desirable that patients with severe heart failure combine home-based programs with periodic (e.g. weekly) outpatient supervised exercise training programs to ensure the safety and compliance. ${ }^{184}$

\section{4) Efficacy Evaluation}

The efficacy of exercise training should be evaluated at months 3 and 6 on the basis of clinical findings, exercise capacity, cardiac function and blood tests, among other measures. Healthcare professionals should provide the results of efficacy evaluation to patients and make them understand the benefits of exercise training to motivate them to continue their lifestyle management strategies. During the maintenance phase, that is defined month 6 and thereafter, healthcare professionals should encourage patients to continue exercise training and maintain a good condition.

\section{5) Patient Education and Counseling}

To accomplish successful exercise training, patients should fully understand how to manage chronic heart failure and acquire practical skills. Healthcare professionals should (1) provide correct knowledge about heart failure (e.g., pathophysiology, triggers and initial symptoms of exacerbation, coronary risk factors); (2) motivate patients to improve their lifestyle to prevent recurrence and inform them to ensure they take appropriate measures (e.g., comply with diet therapy, instruction on drug treatment, and learn self-pulse-taking procedures, how to prevent exacerbation of heart failure); and (3) instruct patients and their family members of the allowance of ADL. Ensuring daily body weight measurement is particularly important.

\section{(4) Cardiac Rehabilitation and Disease Management Programs in Chronic Heart Failure}

In Western countries, an increasing number of reports have described that disease management programs using multidisciplinary interventions including patient education, instruction, counseling, and follow-up after discharge improve QOL and save healthcare cost, and decrease rehospitalization. ${ }^{188-190}$ Disease management programs that cover from the acute to chronic phases of heart failure may be developed by ensuring smooth transition from clinical pathways in acute-phase heart failure to recovery-phase cardiac rehabilitation and home-based management programs in chronic stable heart failure. It is expected that outpatient cardiac rehabilitation programs will be established for long-term management of heart failure.

\section{ICD, CRT-D and Sleep-Disordered Breathing}

\section{(1) Patients With ICD or CRT-D}

\section{Class I}

1. Exercise training aiming to improve exercise capacity and QOL is recommended for heart failure patients treated with ICD. (Level of Evidence: B)

2. Exercise training aiming to further improve exercise capacity and QOL is recommended for heart failure patients treated with CRT. (Level of Evidence: B)

\section{Class IIa}

1. Exercise training aiming to further improve cardiac function is appropriate for heart failure patients treated with CRT. (Level of Evidence: B)

Patients with ICD or CRT-D often experience impaired exercise capacity caused by physical deconditioning due to prolonged bed rest, and a deterioration of their daily QOL due to a fear of the ICD shock. It is concerned that the increase in heart rate associated with exercise may trigger an inappropriate discharge of ICD, but studies have reported that exercise training in patients with ICD is as effective as in patients without ICD and does not cause inappropriate discharges. ${ }^{191}$ It also has been reported that exercise training improves not only exercise capacity but also endothelial function in patients with ICD,${ }^{192}$ and that cardiac rehabilitation relieves anxiety and depression in patients with ICD as compared with findings for those not receiving cardiac rehabilitation. ${ }^{193}$

Although high-level evidence is limited regarding the efficacy and safety of cardiac rehabilitation in patients with ICD, it has been indicated that supervised exercise training in patients with ICD may be conducted safely, and is as effective as that in those without ICD, and have a favorable psychological effect.

It has been well known that CRT devices improve cardiac function, exercise capacity, and QOL. Exercise training further improves exercise capacity in patients with CRT. ${ }^{192}$ In a randomized study in 52 men in chronic heart failure with ischemic cardiomyopathy who received an ICD with or without CRT (i.e., CRT-D or ICD), patients were randomized into 2 groups with and without a supervised exercise training program. The beneficial effects of exercise were especially great among patients with CRT-D. In a RCT to study the effect on exercise capacity of endurance training in addition to CRT in patients with chronic heart failure and dyssynchrony, the increase in peak $\dot{\mathrm{VO}}_{2}$ and maximal workload (Wattmax) was significantly greater in trained versus untrained CRT patients. ${ }^{194}$ In a recent randomized controlled study, patients were allocated into the exercise training or control group 3 months after CRT implantation. Three months after CRT implantation there were significant improvements in NYHA classification, peak $\dot{\mathrm{V}} \mathrm{O}_{2}$, exercise hemodynamics, and cardiac function. After randomization, the exercise group showed further significant improvements in functional, exercise hemodynamic, QOL measures, and skeletal muscle function compared with findings for the control group. ${ }^{195}$ These findings indicate that the addition of cardiac rehabilitation to CRT further improves exercise capacity, cardiac function and other outcome measures in patients with chronic heart failure. To maximize the benefits of CRT, physicians should prescribe cardiac rehabilitation proactively at an appropriate time after CRT implantation.

\section{(2) Patients With Sleep-Disordered Breathing Associated With Heart Failure}

\section{Class I}

1. Exercise training aiming to improve exercise capacity and QOL is recommended for patients with sleep-disordered breathing (SDB) associated with heart failure. (Level of Evidence: C)

\section{Class IIb}

1. The use of non-invasive positive-pressure ventilation aiming to improve exercise capacity and physical functions may be considered for patients with central sleep apnea (CSA) associated with heart failure. (Level of Evidence: C) 
2. Nocturnal home oxygen therapy (HOT) aiming to improve exercise capacity and physical functions may be considered for patients with CSA associated with heart failure. (Level of Evidence: C)

3. Cardiac rehabilitation may be considered to improve SDB in patients with heart failure. (Level of Evidence: C)

It is known that SDB often develops in patients with heart failure. SDB is classified into obstructive sleep apnea (OSA) due to airway obstruction and CSA due to loss of respiratory drive from the respiratory center. It is believed that CSA associated with heart failure and exertional oscillatory ventilation (EOV) that may develop during exercise in patients with heart failure share common pathophysiological characteristics. ${ }^{196}$ It has also been reported that a combination of CSA and EOV has a prognostic value. ${ }^{197}$

\section{1) Improvement in Exercise Capacity With Treatment of Sleep-Disordered Breathing}

It has been reported that treatment of SDB improves exercise capacity in patients with heart failure. For instance, adaptive servo-ventilation (ASV), the most effective treatment method for CSA with Cheyne-Stokes respiration (CSR-CSA), increases peak $\dot{\mathrm{VO}}_{2}$, AT level, and 6-minute walking distance as compared with continuous positive airway pressure (CPAP) $)^{198}$ and nocturnal HOT improves SDB as well as exercise outcome measures such as peak $\mathrm{VO}_{2}$ and AT level. ${ }^{199}$ However, studies reporting such findings are not randomized control studies.

Studies have reported that cardiac rehabilitation improves SDB. In a study of 25 patients with heart failure complicated with SDB, cardiac rehabilitation improved exercise capacity and QOL in patients with and without SDB, and lessened the severity of OSA. ${ }^{200}$

Thus, studies suggest that cardiac rehabilitation may improve SDB. However data of RCTs are limited, and evidence is insufficient (Class IIb).

\section{New Treatment Methods and Cardiac Rehabilitation (1) Surgical Treatment: Mitral Reconstructive Surgery Class IIa}

1. Cardiac rehabilitation is an appropriate intervention for patients who underwent mitral reconstructive surgery for the treatment of heart failure. (Level of Evidence: C)

Mitral reconstructive surgery is a surgical treatment option for patients with heart failure. Patients with ischemic heart failure often undergo $\mathrm{CABG}$ combined with mitral reconstructive surgery. It has been reported that LVEF increased by about $25 \pm 10 \%$ and NYHA classification increased from $3.9 \pm 0.3$ to $2.0 \pm 0.6$ in patients successfully treated with the combination surgery. ${ }^{201}$ However, studies indicate that successful surgery does not promptly improve cardiac function, and exercise capacity does not improve 6 months after the mitral reconstructive surgery alone. ${ }^{202}$ Patients should undergo cardiac rehabilitation aiming to facilitate recovery from cardiac surgery and to improve chronic heart failure.

Exercise training in patients who underwent mitral reconstructive surgery should begin with resistance training that does not affect the sternum. Patients should begin exercise with leg training using a rubber tube or ball on the bed. When standing is allowed, patients should begin with standing on tiptoe on the bedside floor. After the bedside exercise, patients should follow exercise training programs for heart failure.

Safety analysis of patients who underwent mitral reconstructive surgery revealed that no patients experienced new onset or recurrence of mitral regurgitation. ${ }^{203}$

\section{(2) Waon Therapy}

\section{Class I}

1. Waon therapy is effective in patients with heart failure. (Level of Evidence: C)

2. A combination of waon therapy and exercise training is effective. (Level of Evidence: C)

In waon therapy, a thermal therapy developed in Japan, patients stay in a far-infrared heating sauna at $60^{\circ} \mathrm{C}$ for $15 \mathrm{~min}$ utes to increase deep body temperature by $1.0 \sim 1.2^{\circ} \mathrm{C}$, and are covered with thermal blankets to maintain body temperature for 30 minutes. After the session, patients take water to replenish water loss from sweating. Waon therapy with one session a day, 3 5 days a week for 2 6 weeks has been reported effective for increasing cardiac output, ${ }^{204}$ relieving symptoms, enhancing vascular endothelial cell function, activating autonomic nervous system, exerting a favorable effect on neurohumoral factors, alleviating depressive state ${ }^{205}$ and decreasing ventricular arrhythmia in patients with heart failure. ${ }^{206}$ It has been reported that a combination of exercise training and waon therapy improved exercise capacity in patients with chronic heart failure.$^{207}$ It is expected that waon therapy may facilitate exercise training by improving heart failure and decrease arrhythmia.

\section{Current Status and Challenges of Exercise Training Program}

In Japan, chronic heart failure with (1) a LVEF of $\leq 40 \%$ or (2) a blood BNP level of $\geq 80 \mathrm{pg} / \mathrm{mL}$, or (3) a peak $\dot{\mathrm{VO}}_{2}$ of $\leq 80 \%$ of reference value was approved as an indication of cardiovascular rehabilitation in the medical service fee revision in April 2006.

Exercise training in chronic heart failure should be further investigated to (1) establish the criteria for selecting patients who might benefit; (2) establish optimal exercise prescriptions; (3) assess possible synergistic effects of combining exercise training with drug or device treatment; (4) evaluate the effects of exercise training on the prognosis of patients with heart failure in Japan; (5) establish measures to improve adherence to exercise training; and (6) design strategies to encourage exercise training in hospitals treating patients with heart failure.

\section{Heart Transplant Recipients}

Class I

None.

\section{Class Ila}

1. It is appropriate to introduce exercise training early after heart transplantation aiming to improve exercise capacity. (Level of Evidence: B)

The cardiovascular response to exercise is influenced by the loss of autonomic control of heart rate after heart transplantation. Patients may also experience cardiac dysfunction due to donor-to-recipient graft size mismatch or rejection, adverse effects of immunosuppressants, severe deconditioning due to long-term heart failure or prolonged bed rest, and significant anxiety about the future, among others. Healthcare professionals should consider these points carefully before prescribing exercise training programs. 


\section{Efficacy of Exercise Training}

Exercise training increases peak $\mathrm{VO}_{2}$ and decreases resting heart rate in heart transplant recipients. ${ }^{208-211}$ The efficacy of exercise training after heart transplantation has been demonstrated in RCTs. ${ }^{212}$

\section{Cardiac Rehabilitation Programs in Heart Transplant Recipients}

Cardiac rehabilitation in heart transplant recipients consists of the acute, recovery, and maintenance phases.

During the acute phase, cardiac rehabilitation should begin as soon as possible after transplant surgery to avoid complications due to prolonged bed rest and alleviate mental stress.

During the recovery phase, rehabilitation programs that are usually prescribed for patients who underwent cardiac surgery and for those with heart failure should be introduced to improve exercise capacity, expand the ADL, decrease the incidence of mental disorders such as anxiety, depression, and loss of self-confidence, and promote returning to work with better physical and mental health. At the time of patient discharge, physicians in cardiac rehabilitation team should prescribe home-based exercise training programs for recipients and explain how to continue exercise after discharge, and provide instructions for ADL. Recipients should be encouraged to attend outpatient exercise training programs whenever possible.
During the maintenance phase, recipients should continue non-supervised home-based exercise training programs.

\section{Rehabilitation in Patients With Left Ventricular Assist Devices}

Because patients with LVADs or left ventricular assist system (LVAS), who have had heart failure for a long time before LVAD implantation often have severe deconditioning, it is important to help them efficiently recovery from disuse atrophy. Healthcare professionals must have extensive knowledge and understanding of LVADs and motor impairments because LVAD may increase the risk of cerebrovascular disorders resulting from thrombi in the blood pump or bleeding caused by anticoagulation therapy. Reports in and outside Japan indicate that cardiac rehabilitation including early ambulation is necessary for patients with stable hemodynamics after LVAD implantation, and that quantitative aerobic exercise training such as pedaling of cycle ergometer or treadmill walking should be conducted in addition to walking around the ward to promote the recovery of exercise capacity. ${ }^{213,214}$

Because implantable LVADs have recently become covered by the NHI, the use of LVADs will be increasingly common as a measure to facilitate home-based treatment and returning to work. Cardiac rehabilitation will become more important in this patient population. ${ }^{215,216}$

\section{Exercise Training in Children With Cardiac Diseases}

\section{Class I}

None.

\section{Class Ila'}

1. It is appropriate to provide exercise training in children after surgery for congenital heart disease (CHD) as it effectively improves physical functions such as exercise capacity and stroke volume.

However, physicians should carefully assess the effects of exercise training, which may differ significantly among children after surgery for CHD. (Level of Evidence: B)

Exercise during childhood is effective not only in enhancing physical and mental development, maintaining health, and improving QOL but also in promoting the development of a lifelong active lifestyle, preventing obesity and dyslipidemia, and coping with stresses. It is believed that children with CHD should exercise within their limits.

Although a large number of reports have been published on the efficacy of exercise training in children after surgery for CHD, only a few reports have described the effects of exercise training and comprehensive programs in children with CHD before surgery.

\section{Children After Surgery for Congenital Heart Disease}

After surgery for CHD, children who have impaired exercise capacity and abnormal cardiovascular response during exercise should begin exercise training to (1) improve exercise capacity to allow them to exercise safely and improve QOL; (2) encourage them to participate in social and productive activities; and (3) motivate them to continue to exercise, there- by modifying coronary risk factors such as hypertension, diabetes, and hyperlipidemia in the future.

Supervised exercise training is indicated for children with CHD without serious ventricular dysfunction or arrhythmias who cannot undergo further surgical treatment and have impaired exercise capacity. This category of children with CHD include those who have cardiac dysfunction after Rastelli operation, Mustard/Senning operation, or Fontan operation, and those after Glenn operation or Blalock-Taussig operation for whom no further operations are planned, and those who have pulmonary hypertension or arrhythmias after surgery. Children with complete recovery of cardiac function are also indicated for supervised exercise training when they have significantly impaired exercise capacity, feel strong anxiety about exercise, or have been under an unnecessarily strict limitation on exercises. CHD differs significantly between patients, and the cardiac function and cardiopulmonary responses during exercises may often differ even among children who have a similar type and severity of CHD and lesions remaining after surgery.

Safe exercise prescriptions are especially important for children with severe CHD. Physicians should carefully and comprehensively evaluate the characteristics of cardiopulmonary responses during exercise in each child before prescribing exercise training programs. Exercise intensity should be set according to the results of an exercise stress test and the condition and characteristics of the individual patient. Physicians should carefully consider the risk and history of significant ventricular dysfunction and arrhythmias. It should also be noted that the physical development and associated changes in physical activities after the introducing of exercise training may affect the evaluation of efficacy of training programs in children.

The efficacy of exercise training in children has been re- 
ported in studies using programs consisting of 60-minute sessions including 20 30 minutes main exercise, 2 3 sessions/ week, for 10 12 week. The intensity of the main exercise is generally set at $60 \sim 80 \%$ of the maximum heart rate. However, it is more desirable that children should begin their programs with an intensity of around the AT, be followed for changes over time in exercise capacity, and undergo a gradual increase towards more intense training for longer durations.

It is important to provide enjoyable and game-like exercise sessions to prevent dropout and motivate children to continue their exercise programs. Parents should also be encouraged to join exercise training programs to motivate the children to continue their programs, help the families establish active lifestyles, and improve QOL of the children and family members after completing the exercise training programs. ${ }^{217-219}$

\section{Children With Congenital Heart Disease Who Have Not Undergone Surgery}

It is known that children with acyanotic CHD, except for those with severe disease, show exercise capacity similar to healthy children in treadmill exercise test. Exercise should be restricted in children with uncorrected aortic stenosis, mitral regurgitation, or aortic regurgitation.

Children with cyanotic CHD have hypoxemia that causes impaired exercise capacity and dyspnea with even the slightest activity, and limits spontaneous exertion. However, it has been reported that exercise training may improve exercise capacity even in children with cyanotic CHD through increasing cardiopulmonary function and muscle strength. Because physical fitness does not improve in children with a less active lifestyle, children are recommended to perform exercises within their limits. However, it is unclear whether exercises within the patient's physical limitations may improve cardiopulmonary function or not. 220,221

\section{Problems and Future Challenges in Exercise Training Programs for Children With Congenital Heart Disease}

The efficacy of exercise training programs for children with $\mathrm{CHD}$ varies among hospitals. This difference is believed to be caused by the diversity in the children relating to their age, cardiac function, and exercise capacity at baseline, as well as the differences in type and severity of CHD and in lesions remaining after surgery. In addition, study protocols and exercise treatments will differ between hospitals. Further studies should be conducted to develop criteria for selecting children with CHD who are expected to respond favorably to exercise training, to investigate how to prescribe exercise training programs, to clarify mechanisms of the effect of exercise training on the cardiac function of children with $\mathrm{CHD}$, and to develop optimal and effective exercise programs for children with severe CHD. ${ }^{222}$

\section{Significance of Exercise Training in Elderly Patients With Cardiovascular Diseases}

In the guidelines on secondary prevention of coronary heart disease in the elderly (with emphasis on patients $\geq 75$ years of age), the ACCF and the AHA recommend that coronary risk factors in elderly patients should be managed similarly to those in younger patients. ${ }^{223}$

As Table 29 shows, elderly patients with cardiovascular disease have many complications and poor physical functions that often limit participation in cardiac rehabilitation programs. However, a number of studies have reported that the beneficial effect of cardiac rehabilitation programs on elderly patients are similar to those on younger patients. ${ }^{224,225}$ The results of these studies in elderly patients were fairly consistent with the results of RCTs and meta-analyses of studies of cardiac rehabilitations in younger patients (Tables 30 and 31).226-230

\section{Significance of Exercise Training in Elderly Patients (Table 32)}

It has been reported that exercise training improves physical functions and QOL in elderly patients with cardiovascular diseases. ${ }^{231-233}$ Reports have also described that exercise train-

Table 29. Characteristics of Elderly Patients With Cardiovascular Disease

1. Women are more common as compared with the sex ratio in younger patients.

2. The number of cardiac surgery and the incidence of heart failure is high.

3. Patients with underlying diseases (e.g., carotid atherosclerosis, arteriosclerosis obliterans, chronic respiratory failure, chronic kidney disease, stroke, cognitive disorder, and orthopedic disorders) are common.

4. Many patients cannot continue cardiac rehabilitation programs due to the conditions such as atrial fibrillation, cognitive disorder, anemia, acute exacerbation of chronic kidney disease, massive pleural effusion requiring drainage, respiratory support, and infections.

5. The rate of discharge to home is low: social resources should be applied.

Table 30. Characteristics of Elderly Patients With Acute Myocardial Infarction (AMI)

1. The rate of participation in cardiac rehabilitation decreases as the age increase. The rate is low among elderly patients and women of all ages.

2. The mortality and incidence of recurrent myocardial infarction are low among patients participating in cardiac rehabilitation after AMI. Survival rate among this patient population is not higher than the reference population. 
Table 31. Effects of Cardiac Rehabilitation Programs Among Patients Over 65 Years Old With Coronary Artery Disease

1. Better management of coronary risk factors

2. Prevention of cardiovascular events

3. Decrease in mortality rate

Table 32. Effects of Aerobic Exercise and Resistance Training

1. Improvement in muscle strength

2. Decrease in the incidence of orthostatic hypotension

3. Improvement in vision

4. Improvement in exercise capacity (peak $\dot{\mathrm{VO}}_{2}, \mathrm{AT} \dot{\mathrm{VO}}_{2}, 6$-minute walking distance: $\mathrm{MD}$, walking and stair climbing)

5. Improvement in coronary risk factors (e.g., lipid metabolism, blood pressure, and body composition)

6. Improvement in autonomic nervous system function (QTc interval, heart rate variability, and BRS)

7. Improvement in vascular endothelial function

8. Improvement in mental/psychological factors such as health-related QOL and depression

9. Decrease in proinflammatory cytokine levels in patients with heart failure

10. Improvement in cardiac function

AT $\dot{\mathrm{VO}}$, oxygen uptake at anaerobic threshold; BRS, baroreflex sensitivity; peak $\dot{\mathrm{VO}}_{2}$, peak oxygen uptake; MD minutes distance.

\begin{tabular}{|c|c|}
\hline \multicolumn{2}{|l|}{ Aerobic exercise } \\
\hline Intensity & $\begin{array}{l}-60 \% \text { of peak } \mathrm{VO}_{2} \\
-50 \% \text { of maximum exercise intensity } \\
-70 \% \text { of maximum heart rate } \\
-\mathrm{AT} \\
\text { - Borg scale of } 11 \sim 13 \text { (RPE: fairly light } \sim \text { somewhat hard) }\end{array}$ \\
\hline Duration & $20 \sim 60 \mathrm{~min} / \mathrm{session}$ \\
\hline Frequency & 2 5 sessions/week \\
\hline \multicolumn{2}{|l|}{ Resistance training } \\
\hline Intensity & $40 \sim 60 \%$ of $1 \mathrm{RM}$ \\
\hline Number of repetitions & $\begin{array}{l}\text { One set consists of 12 15 repetitions } \\
\text { One session consists of } 2 \sim 3 \text { sets }\end{array}$ \\
\hline Frequency & 3 sessions/week \\
\hline \multicolumn{2}{|l|}{ Interval training } \\
\hline Intensity & $\begin{array}{l}\text { 1. Vigorous: } 90 \sim 95 \% \text { of maximum heart rate } \\
\text { Light: } 50 \sim 70 \% \text { of maximum heart rate } \\
\text { 2. Increase exercise intensity to Borg scale of } 18 \text { (RPE: very, very hard) in } 5 \sim 10 \text { minutes, } \\
\text { then decrease gradually to } 10 \text { watts. }\end{array}$ \\
\hline Duration & $\begin{array}{l}\text { 1. Interval training consists of } 4 \text {-minute vigorous exercise followed by } 3 \text {-minute light } \\
\text { exercise for a total of } 20 \sim 25 \text { minutes. } \\
\text { 2. Conduct } 3 \text { sets of interval training for a total of } 40 \sim 50 \text { minutes. }\end{array}$ \\
\hline Frequency & 3 sessions/week \\
\hline
\end{tabular}

1RM, one repetition maximum; AT, anaerobic threshold; peak $\dot{\mathrm{VO}}_{2}$, peak oxygen uptake; RPE, ratings of perceived exertion.

ing significantly prolonged the duration of continuous exercise in elderly patients with heart failure, ${ }^{75,234,235}$ and that exercise training that started in the early phase after hospitalization shortened the duration of hospital stay, decreased the frequency of rehospitalizations, and reduced healthcare cost in elderly patients with MI. It also has been reported that home-based exercise training programs are beneficial in maintaining exercise capacity and reducing healthcare costs in low-risk patients after MI. ${ }^{236,237}$ There has been increasing evidence from large- scale epidemiological studies and RCTs on the safety of exercise training and the role of comprehensive programs for elderly patients with cardiovascular diseases. ${ }^{228,238-241}$ Elderly patients should be strongly encouraged to participate in cardiac rehabilitation programs.

\section{Class I}

1. Aerobic exercise programs is recommended for elderly patients with CAD and those with heart failure. (Level of 


\section{Evidence: A)}

2. Exercise intensity in aerobic exercise is recommended to be moderate (around the AT) in elderly patients with CAD. (Level of Evidence: A)

\section{Class Ila}

1. It is appropriate that exercise prescriptions for elderly patients with CAD include outpatient supervised and homebased non-supervised exercise training. (Level of Evidence: B)

\section{Class Ila'}

1. It is appropriate that exercise training programs for elderly patients with CAD or heart failure consist of aerobic training and resistance training. It is appropriate that nutritional therapy and resistance training be prescribed for patients with reduced muscle mass. (Level of Evidence: $\mathbf{C}$ )

2. It is appropriate that the intensity of resistance training for elderly patients with CAD would be moderate, i.e., 40 60\% of one repetition maximum (1RM). (Level of Evidence: B)

\section{Exercise Training in Elderly Patients With Cardiovascular Diseases}

\section{Exercise Prescriptions (Table 33)}

\section{Places}

After patients experienced supervised exercise training in medical institutions, home-based training programs should be added and increased as the main component of continuing exercise.

There might be a concern that elderly patients with heart failure are at a high risk for developing cardiovascular events during exercise. Although few report regarding cardiovascular events during exercise has been published in elderly patients with heart failure, elderly as well as younger patients should begin with supervised exercise training in medical institutions first to confirm the patients' response to exercise.

\section{Exercise Training for Large Vessel and Peripheral Arterial Diseases}

\section{Rehabilitation in Patients With Large Vessel Diseases}

\section{Class I}

None.

\section{Class Ila'}

1. It is appropriate that patients planning to undergo large vessel surgery perform exercise training to enhance respiratory function; this should be performed during blood pressure monitoring. (Level of Evidence: C)

2. Cardiac rehabilitation after large vessel surgery is an appropriate intervention as it may decrease the duration of

Table 34. Effects of Rehabilitation Programs for Patients After Large Vessel Surgery

1. Rehabilitation is expected to decrease hospital days by improving cardiopulmonary function and strengthening muscles. ${ }^{245}$

2. Rehabilitation decreases the incidence of postoperative complications (e.g., infections, pneumonia, pleural effusion, and delirium).

3. Rehabilitation improves prognosis and QOL. ${ }^{246}$

Table 35. Rehabilitation by Type of Aneurysm

Type of aneurysm

True aneurysm

Aortic dissection after surgery

No residual dissection or false lumen

Presence of residual dissection or false lumen

Pseudo aneurysm

\section{Management}

Basic postoperative rehabilitation programs

Basic postoperative rehabilitation programs

Individualized management and more strict blood pressure control are required.

Appropriate management varies by the cause of pseudo aneurysm (e.g., injuries and Behcet's disease). After radical surgery, patients may participate in rehabilitation programs similarly as do patients with true aneurysm.

Table 36. Rehabilitation by Location of Aneurysm

Location

Thoracic aortic aneurysm

Ascending aorta/arch

Descending aorta

Abdominal aortic aneurysm

\section{Affected organ systems/ pain location}

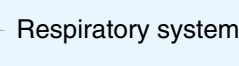

Bowel system

\section{Conditions requiring management to improve ADL after surgery}

Conditions such as swallowing disorders and residual dissection Control of pleural effusion

Gastrointestinal symptoms such as anorexia (dietary habit before surgery is useful information)

$A D L$, activities of daily living. 
Table 37. Points for Rehabilitation by Cause of Aneurysm

\section{Cause}

Atherosclerosis

- Most common cause of aneurysm.

- Common in elderly patients.

- Prevention of disuse syndrome is important.

- Because systemic complications are commonly observed, patients should be observed for cerebral ischemia, ischemic heart disease, renal ischemia, and arteriosclerosis obliterans (leg ischemia).

Marfan's syndrome - Relatively common in younger patients.

- Concomitant non-cardiac atherosclerotic disease is rare, but tissues are fragile.

- It has been reported that angiotensin II receptor blockers significantly slowed the rate of progressive aortic-root dilation. ${ }^{247}$

\section{Table 38. An Example of Stepwise Rehabilitation After Endovascular Stent Grafting}

Date

Day of stenting

Day 1 after stenting

$100 \mathrm{~m}$ walking test

Day 2 after stenting $\quad 300 \sim 500 \mathrm{~m}$ walking test

\section{Exercise}

Target rate: $2 \mathrm{mph}$ (about $54 \mathrm{~m} / \mathrm{min}$ ) (about 2.0 2.5METs)

*If difficult, start walking at the patient's own pace.

Require nurse aide during first test.

METs, metabolic equivalents.

\section{Level of activity}

Bed rest

When walking test is passed, the patient is allowed to walk in the ward freely. However, long-distance walking is not recommended. Walking should be limited to going to the toilet.

When walking test is passed, the patient is allowed to walk in the hospital freely.

\section{Table 39. Characteristics of Patients With Aortic Disease by Location}

Thoracic aortic aneurysm (TAA)

- More common in $\leq 60$ years old.

- Saccular aneurysm accounts for about $30 \%$ of cases.

- The most common location is the arch for saccular aneurysm and the descending aorta for fusiform aneurysm.

- Male: female ratio is about 3 4:1.

Thoracoabdominal aortic aneurysm

- More common among patients in their 50 60s (Younger than patients with TAA).

- Male: female ratio is about $4: 1$.

\section{Abdominal aortic aneurysm (AAA)}

- Most common among patients in their 60 70s.

- Results of surgery in elderly patients are as good as younger patients.

*However, the prognosis is poor in patients $\geq 80$ years old, male patients, patients with chronic renal failure, patients with peripheral arterial disease, and patients undergoing emergency surgery.

- Male: female ratio is $5 \sim 8: 1$.

- Prognosis is poor in women.

- The prevalence and mortality of ruptured aneurysm is higher in women than that seen in men. Inflammatory

- Inflammatory AAA accounts for about $4 \%$ of all AAA.

- Male: female ratio is 6:1.

Aortic dissection (including dissecting aortic aneurysm)

- Common among patients in their 50 60s.

- Male: female ratio is about 3:1.

- About half of female patients are $\geq 70$ years old. Mortality due to aortic dissection is higher in women than that seen in men.

hospital days, result in early return to society and a high rate of successful returning to work, improve prognosis and QOL, and enhance postoperative recovery in elderly patients by enhancing cardiopulmonary functions and strengthening muscles. (Level of Evidence: C)
1. Current Practice of Rehabilitation Programs for Patients After Large Vessel Surgery

Early introducing of rehabilitation programs for patients after large vessel surgery is increasingly common, and has facilitated early discharge from hospitals. ${ }^{242}$

Patients with acute aortic dissection are undergoing rehabilitation according to the recommendations on cardiac rehabilitation programs. ${ }^{243,244}$ 
Table 40. Items to Be Monitored and Assessed During Rehabilitation After Large Vessel Surgery

1. Blood pressure control (maintain optimal blood pressure)

- Maintain systolic blood pressure at $\leq 130 \mathrm{mmHg}$.

2. Exercise stress test

- Expand activity serially to sitting, standing, walking around the ward, taking a shower, taking a bath, and physical exercise.

- In addition to walking, exercise tests may include treadmill and cycle ergometer exercises.

- Target systolic blood pressure is $\leq 130 \mathrm{mmHg}$ before exercise and $<150 \mathrm{mmHg}$ during exercise.

3. 24-hour blood pressure monitoring

4. QOL survey

- QOL survey is recommended as it helps with prescribing and revising/refining exercise training programs.

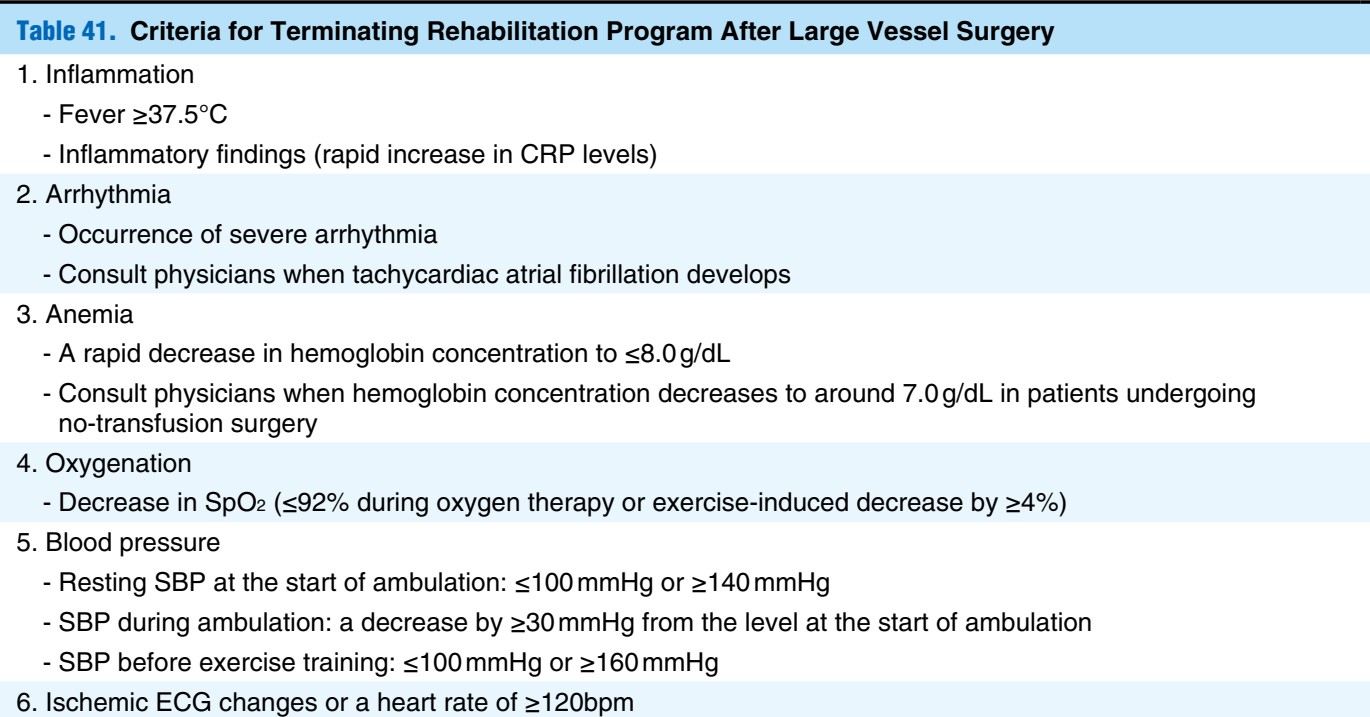

$\mathrm{CRP}, \mathrm{C}$ reactive protein; $\mathrm{SBP}$, systolic blood pressure; $\mathrm{SpO}_{2}$, peripheral arterial oxygen saturation.

Source: Guidelines for Rehabilitation in Patients with Cardiovascular Disease (JCS2007), ${ }^{251}$ and Watanabe S, et al.

The inhibiting factors of physical therapy in aortic dissection repair. Physical Therapy Japan 2005; 32: 72-76.252

2. Effects of Rehabilitation Programs for Patients After Large Vessel Surgery (Table 34)245,246

3. Conditions to Be Considered When Prescribing Rehabilitation Programs for Patients After Large Vessel Surgery

(1) Rehabilitation Programs by Type of Aortic Aneurysm (Table 35)

(2) Rehabilitation Programs by Location of Aortic Aneurysm (Table 36)

(3) Rehabilitation Programs by Cause of Aortic Aneurysm (Table 37) 247

\section{(4) Rehabilitation Programs by Treatment Method of} Aortic Aneurysm

Endovascular stent grafting is a minimally invasive technique for the treatment of aortic aneurysm and dissection (Table 38). After stenting, patients may be able to start walking early after surgery, be discharged from the hospital 7 days after stenting, and live as usual when return home.

Target systolic blood pressure and heart rate in patients after large vessel surgery are $\leq 130 \mathrm{mmHg}$ and $\leq 70 \mathrm{bpm}$ at rest, and $\leq 140 \mathrm{mmHg}$ and $\leq 90 \mathrm{bpm}$ during exercise, respectively. ${ }^{248}$ In patients with thoracic aortic aneurysm complicated with spinal cord injury with paraplegia, blood pressure should be maintained at a higher level because a decrease in blood pressure may exacerbate symptoms.

\section{(5) Rehabilitation in Patients Undergoing Elective Surgery for Non-Ruptured Aortic Aneurysm and Patients Undergoing Emergency Surgery for Ruptured Aortic Aneurysm}

The condition and risk management of patients differs between those undergoing elective surgery for non-ruptured aortic aneurysm and those undergoing emergency surgery for ruptured aortic aneurysm. Patients with ruptured aneurysm have poor general condition, often experience postoperative complications, and undergo surgery with no preoperative assessment. After surgery, they participate in cardiac rehabilitation without detailed information on underlying diseases such as CAD, cerebrovascular disorder, and pulmonary disease.

\section{Patient Characteristics by Age and Gender (Table 39)}

Patients after thoracic aortic graft replacement and elderly patients after large vessel surgery may experience swallowing disorders, and should be considered for swallowing assess- 
Table 42. An Example of a Rehabilitation Program for Patients After Large Vessel Surgery

\begin{tabular}{|c|c|c|c|}
\hline & $\begin{array}{c}\text { Patients without residual } \\
\text { dissection }\end{array}$ & $\begin{array}{l}\text { Patients with residual } \\
\text { dissection }\end{array}$ & $\begin{array}{l}\text { Patients with descending thoracic } \\
\text { aortic aneurysm }\end{array}$ \\
\hline $\begin{array}{l}\text { Blood pressure } \\
\text { Stage }\end{array}$ & $\mathrm{SBP} \leq 160 \mathrm{mmHg}$ & $\mathrm{SBP} \leq 140 \mathrm{mmHg}$ & $\mathrm{SBP} \leq 140 \mathrm{mmHg}$ \\
\hline 1 & Start on postoperative day 1 & Complete on postoperative day 7 & Complete on postoperative day 3 \\
\hline II & Start on postoperative day 2 & Complete on postoperative day 14 & Start on postoperative day 3 \\
\hline $\begin{array}{l}\text { III } \\
\text { IV } \\
\text { V }\end{array}$ & $\begin{array}{l}\text { Start on postoperative day } 3 \\
\text { Start on postoperative day } 4 \\
\text { Start on postoperative day } 5\end{array}$ & $\begin{array}{l}\text { Start on postoperative day } 14 \text { with careful } \\
\text { observation for thrombosis of } \\
\text { residual false lumen }\end{array}$ & $\begin{array}{l}\text { Start on postoperative day } 5 \\
\text { with careful monitoring } \\
\text { of oxygenation }\end{array}$ \\
\hline $\begin{array}{l}\text { VI } \\
\text { VII }\end{array}$ & $\begin{array}{l}\text { Start on postoperative day } 6 \\
\text { Start on postoperative day } 7\end{array}$ & Start on postoperative day 21 & Start on postoperative day 10 \\
\hline
\end{tabular}

SBP, systolic blood pressure.

Adapted from Watanabe S. Cardiac surgery and physical therapy for elderly or disabled patients. The Japanese Journal of Physical Therapy 2005; 39: 771-776. ${ }^{254}$

\begin{tabular}{|ccccccc|}
\hline \multicolumn{2}{|c|}{ Table 43. An Example of a Rehabilitation Program for Patients After Abdominal Aortic Aneurysm Surgery (Cardiovascular Center, } \\
Saiseikai Kumamoto Hospital)
\end{tabular}

Adapted from Nishigami K. Rehabilitations for complications of aortic disease. J Jpn Soc Int Med 2010; 99: 87-91.255

\begin{tabular}{|c|c|c|}
\hline Exercise & Methods & Level of activity \\
\hline 2-minute stepping & \multicolumn{2}{|l|}{$\begin{array}{l}\text { Stand up slowly at the bedside for oneself, and step in } \\
\text { place for } 2 \text { minutes }\end{array}$} \\
\hline $50 \mathrm{~m}$ walking & \multirow{4}{*}{$\begin{array}{l}\text { Target rate: } 2 \mathrm{mph} \text { (about } 54 \mathrm{~m} / \mathrm{min} \text { ) (about 2.0 2.5 METs) } \\
\text { *If difficult, start walking at the patient's own pace. }\end{array}$} & \multirow{2}{*}{ When test is passed, allow to use ward toilet } \\
\hline $100 \mathrm{~m}$ walking & & \\
\hline $300 \mathrm{~m}$ walking & & \multirow{3}{*}{ When test is passed, allow to take a shower. } \\
\hline $500 \mathrm{~m}$ walking & & \\
\hline Exercise training room & $\begin{array}{l}\text { Exercise training such as walking, treadmill walking, and cycle } \\
\text { ergometer (low level of exercise intensity is recommended). }\end{array}$ & \\
\hline
\end{tabular}

METs, metabolic equivalents.

ment and training by dentists, speech therapist, or other appropriate health professionals. It is preferable to provide appropriate preoperative approaches and serve gooey food diet after surgery whenever possible to promote the recovery of swallowing function. ${ }^{249}$

5. Rehabilitation for Patients After Large Vessel Surgery

(1) Monitoring and Assessment During Rehabilitation After Large Vessel Surgery (Table 40)

\section{(2) Criteria for Terminating Rehabilitation Programs (Table 41) 250-252}

\section{(3) Stepwise Implementation of Rehabilitation}

1. Patients should receive preoperative training to enhance respiratory function. Patients with abdominal aortic aneurysm $\left(3 \sim 5 \mathrm{~cm}\right.$ in diameter) may undergo CPX safely. ${ }^{253}$

2. After surgery, patients should be encouraged to expand the range of ADL step by step (Table 42). ${ }^{254}$

3. Characteristics of each stage and stepwise process of rehabilitation (Tables $\mathbf{4 3}{ }^{255}$ and 44 )

\section{Treatment and Effects of Rehabilitation on Systemic} Atherosclerotic and Venous Diseases

Inflammation is an important pathophysiological feature of aortic aneurysms. In a double-blind, placebo-controlled trial in patients undergoing vascular surgery (50\% of them underwent abdominal aortic aneurysm repair), statins decreased the incidence of postoperative cardiovascular events. ${ }^{256}$

It has been reported that deep vein thrombosis and pulmo- 
Table 45. Arterial Occlusive Diseases That May Cause Claudication

Causes of occlusive arterial lesions in lower extremity arteries potentially causing claudication

- Atherosclerosis (arteriosclerosis obliterans [ASO])

- Arteritis

- Congenital and acquired coarctation of aorta

- Endo-brosis of the external iliac artery (iliac artery syndrome in cyclists)

- Fibromuscular dysplasia

- Peripheral emboli

- Popliteal aneurysm (with secondary thromboembolism)

- Adventitial cyst of popliteal artery

- Popliteal arterial entrapment

- Primary vascular tumors

- Pseudoxanthoma elasticum

- Remote trauma or irradiation injury

- Takayasu's disease

- Thromboangiitis obliterans (Buerger's disease)

- Thrombosis of a persistent sciatic artery

Adapted from Norgren L, et al. Inter-Society Consensus for the Management of Peripheral Arterial Disease (TASC

II). J Vasc Surg 2007; 45(Suppl S): S5-S67, 258 with permission from Elsevier Inc.

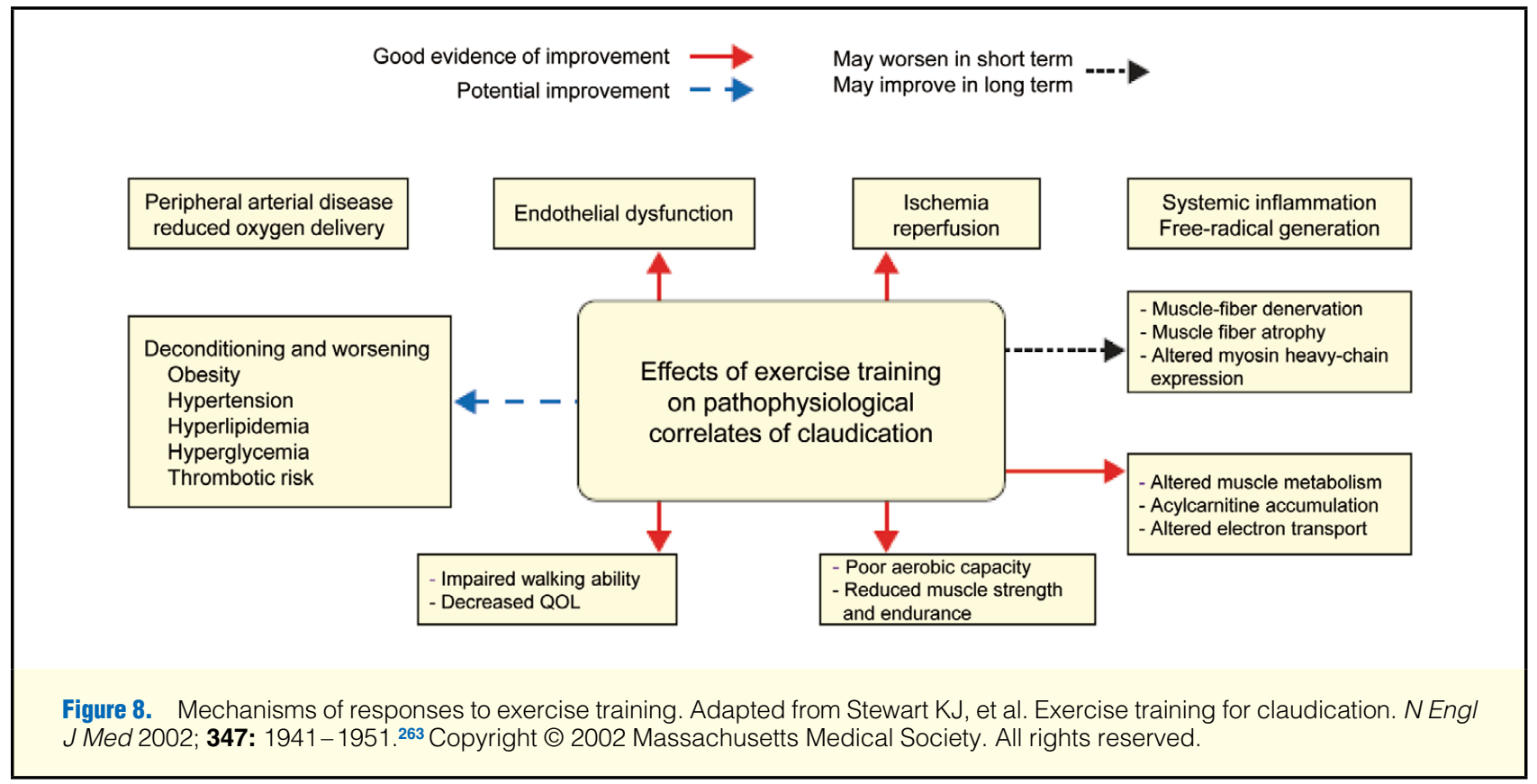

nary embolism developed in $8.1 \%$ of patients after abdominal aortic aneurysm repair. ${ }^{257}$

\section{Peripheral Arterial Rehabilitation in Patients With Chronic Peripheral Arterial Occlusive Diseases}

\section{Class I}

1. Exercise training programs for patients with intermittent claudication is recommended to prescribe according to the presence and severity of ischemia using the ankle brachial pressure index (ABPI). (Level of Evidence: B)

It is also recommended that patients undergo vascular ul- trasound, magnetic resonance (MR), magnetic resonance angiography (MRA) or computed tomography (CT) to investigate the etiology and pathology (location of lesions and severity of stenosis) of intermittent claudication. (Level of Evidence: B)

2. Exercise training, especially supervised exercise training, is recommended for patients with intermittent claudication due to chronic peripheral arterial occlusive disease whenever it is not contraindicated. (Level of Evidence: A)

It is desirable that exercise intensity be prescribed. Exercise machines such as treadmills and ergometers should be used. Use of a walking track equipped with a pacemaker is also acceptable. (Level of Evidence: A)

Exercise programs are recommended to continue for 3 


\begin{tabular}{|c|c|c|c|c|}
\hline \multicolumn{2}{|r|}{ Fontaine } & \multicolumn{3}{|c|}{ Rutherford } \\
\hline Stage & Clinical symptoms & Grade & Category & Clinical symptoms \\
\hline 1 & Asymptomatic & 0 & 0 & Asymptomatic \\
\hline Ila & Mild claudication & I & 1 & Mild claudication \\
\hline \multirow[t]{2}{*}{$\mathrm{llb}$} & Moderate to severe claudication & 1 & 2 & Moderate claudication \\
\hline & & I & 3 & Severe claudication \\
\hline \multirow[t]{2}{*}{ III } & Ischemic rest pain & II & 4 & Ischemic rest pain \\
\hline & & III & 5 & Minor tissue loss \\
\hline IV & Ulceration or gangrene & III & 6 & Major tissue loss \\
\hline
\end{tabular}

Adapted from Norgren L, et al. Inter-Society Consensus for the Management of Peripheral Arterial Disease (TASC II). J Vasc Surg 2007; 45(Suppl S): S5-S67, ${ }^{258}$ with permission from Elsevier Inc.

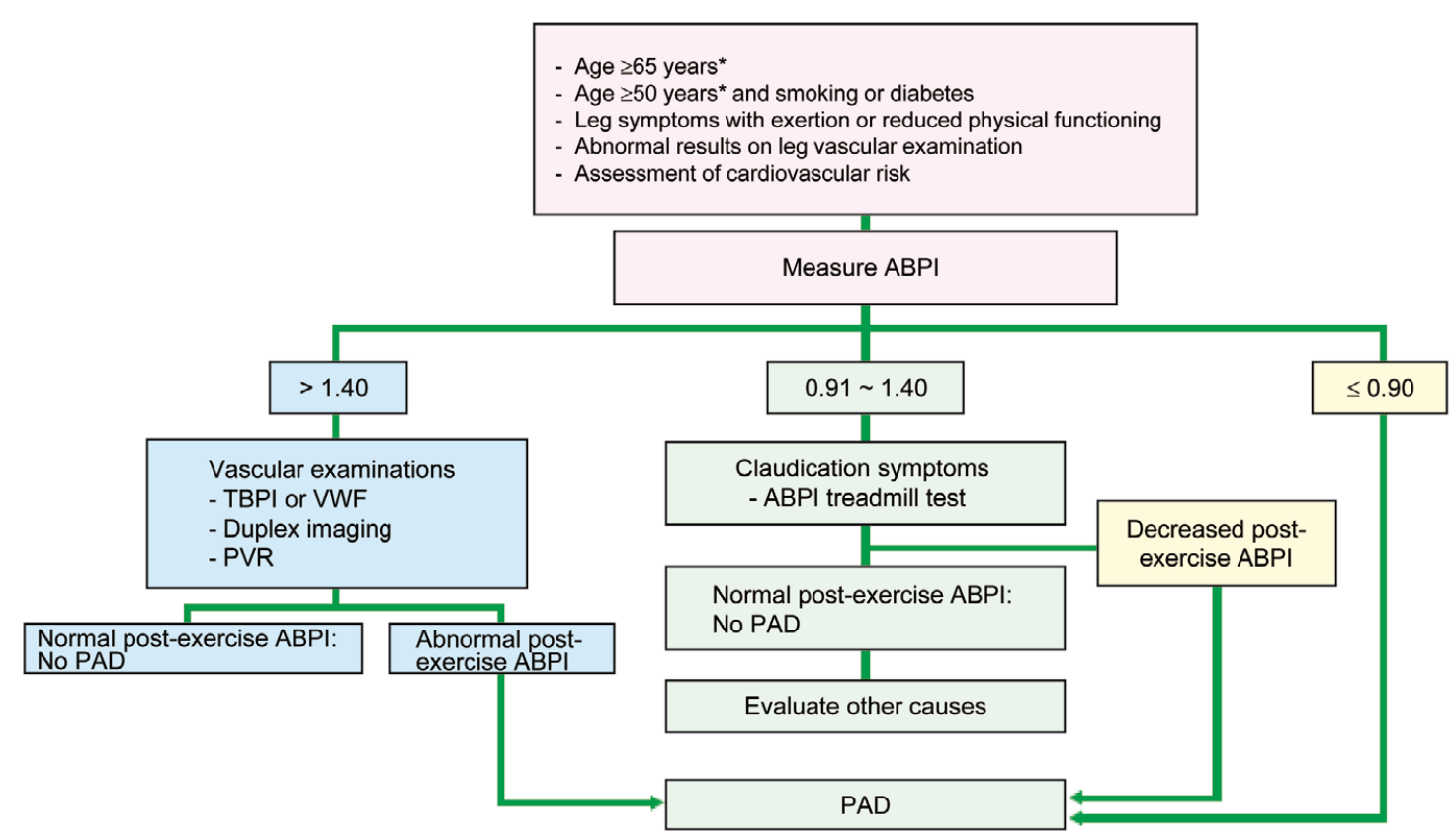

Figure 9. Algorism for diagnosis of peripheral arterial disease (PAD). ABPI, ankle-brachial pressure index; PVR, pulse volume recording; TASC, TransAtlantic Inter-Society Consensus; TBPI, toe brachial pressure index; VWF, velocity wave form. Adapted from Norgren L, et al. Inter-Society Consensus for the Management of Peripheral Arterial Disease (TASC II). J Vasc Surg 2007; 45(Suppl S): S5-S67, ${ }^{258}$ with permission from Elsevier Inc. *Modified in accordance with 2011 ACCF/AHA Focused Update of the Guidelines. ${ }^{265}$

months or longer. (Level of Evidence: A)

3. Patients with arteriosclerosis obliterans (ASO) should be carefully examined for systemic complications and prognosis. Exercise programs should be prescribed after careful assessment for complications of major organs (especially ischemic heart disease). (Level of Evidence: B)

The use of antiplatelet drugs is recommended as a measure to decrease the risk of cardiovascular complications and death in patients with ASO. (Level of Evidence: A)

\section{Class Ila}

1. Combination of drug treatment and home-based exercise training programs is an appropriate regimen for patients with intermittent claudication who cannot participate in supervised exercise training. (Level of Evidence: C)

\section{Introduction}

The type of intermittent claudication for which exercise training is recommended in the present guidelines is ischemic claudication due to chronic obstruction of peripheral arteries. When detailed pathological classification of intermittent claudication is required, descriptions such as "arteriosclerosis obliterans (ASO)" and other types of chronic arterial stenosis/ occlusion such as "Buerger's disease (thromboangiitis obliterans), a chronic inflammatory disease", are made. Table 45 lists arterial occlusive diseases that may cause claudication. ${ }^{258}$ 
- The patient must receive a full explanation on how to walk on the treadmill, and must perform a trial walk as well as take a rest before the examination.

- ECG should be monitored throughout the examination.

- ABPI should be measured before treadmill walking.

- The grade of the treadmill should be 12 degrees, and the walking speed should be $2.4 \mathrm{~km} / \mathrm{h}$.

- The patient should keep a good posture during walking, and should not lean on the handle.

- Pain-free walking distance and maximum walking distance should be determined (Record the sites of pain).

- ABPI should be remeasured immediately after the end of treadmill walking test.

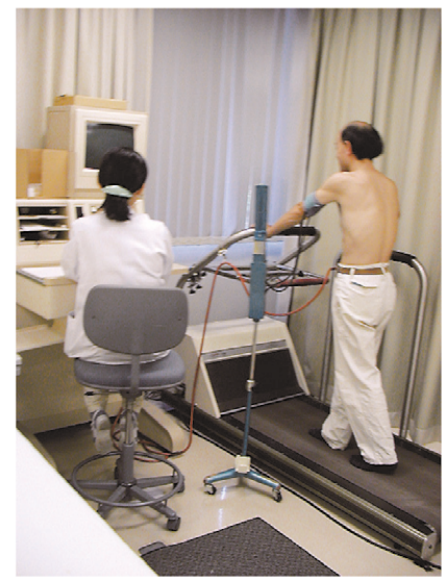

Figure 10. Determination of treadmill walking distance. ABPI, ankle-brachial pressure index (also referred to as $A B I$ and $A P I$ ) Normal range is $0.90 \sim 1.40$. An ABPI of $<0.90$ is considered abnormal.

\begin{tabular}{|c|c|c|}
\hline Fontaine's stage & Clinical findings & Treatment policy \\
\hline 1 & Asymptomatic (cold foot, numbness) & $\begin{array}{l}\text { Eliminate risk factors and prevent the progression of } \\
\text { peripheral arterial disease }\end{array}$ \\
\hline II & Intermittent claudication & $\begin{array}{l}\text { Treatment for type I patients plus exercise training, } \\
\text { drug treatment, and invasive treatment }\end{array}$ \\
\hline $\begin{array}{l}\text { III } \\
\text { IV }\end{array}$ & $\begin{array}{l}\text { Pain at rest } \\
\text { Gangrene or ischemic ulcer }\end{array}$ & Conduct invasive treatment first to salvage limbs \\
\hline
\end{tabular}

\section{Effects of Exercise Training}

\section{(1) Increase in Walking Distance}

Exercise training increases walking distance in patients with ischemic claudication. ${ }^{259}$ In a multicenter study in 53 patients in Japan, exercise training increased walking distance (See Table 51 of the full version of the guidelines). ${ }^{260}$

\section{(2) Effects on Q0L and Prognosis}

- Effects on QOL (MOS 20-item Short Form Health Survey [SF-20], ${ }^{261}$ SF-36, ${ }^{262}$ Walking Impairment Questionnaire [WIQ] ${ }^{252}$ )

- Effects on long-term prognosis ${ }^{247}$

\section{(3) Cost-Effectiveness}

\section{Mechanism of Effects of Exercise Training (Figure 8$)^{263}$}

3. Precautions for Implementation of Exercise Training for Ischemic Claudication

(1) Definitive Diagnosis of Peripheral Arterial Occlusive Disease (Table 46258, Figures 9258,264,265 and 10)

\section{(2) Complication of Systemic Atherosclerotic Diseases}

Patients with ASO due to atherosclerosis may have systemic, for example, cerebral, carotid, coronary, or renal arteries resulting in systemic complications. Patients with ASO should be carefully assessed for systemic condition including complications in major organs (especially coronary heart disease), and prognosis before prescribing exercise training programs for claudication.

\section{(3) Sex Difference}

The male-female ratio of patients with intermittent claudication is $9: 1$. But the incidences differ according to age. The incidence in female patients increases as age increases.

\section{(4) Positioning of Exercise Training in the Treatment of Chronic Peripheral Arterial Occlusive Disease (Table 47)}

Basic treatment strategies for ASO include (1) treatment of peripheral circulatory disturbance, (2) treatment of circulatory disturbance in other organs, and (3) management of risk factors for atherosclerosis.

\section{Exercise Training for Patients With Chronic Peripheral Arterial Occlusive Diseases}

(1) Indications and Contraindications of Exercise Training (Figure 11)266

It has been proposed that $\mathrm{ABPI}$ recovery time after one-minute walk on a treadmill at grade $12 \%$ and a speed of $2.4 \mathrm{~km} / \mathrm{h}$ should be determined before prescribing exercise training programs, and patients with an ABPI recovery time of $\leq 12$ minutes are expected to improve walking distance after exercise training. ${ }^{267}$

A combination with supervised exercise training after bypass surgery is also recommended. 


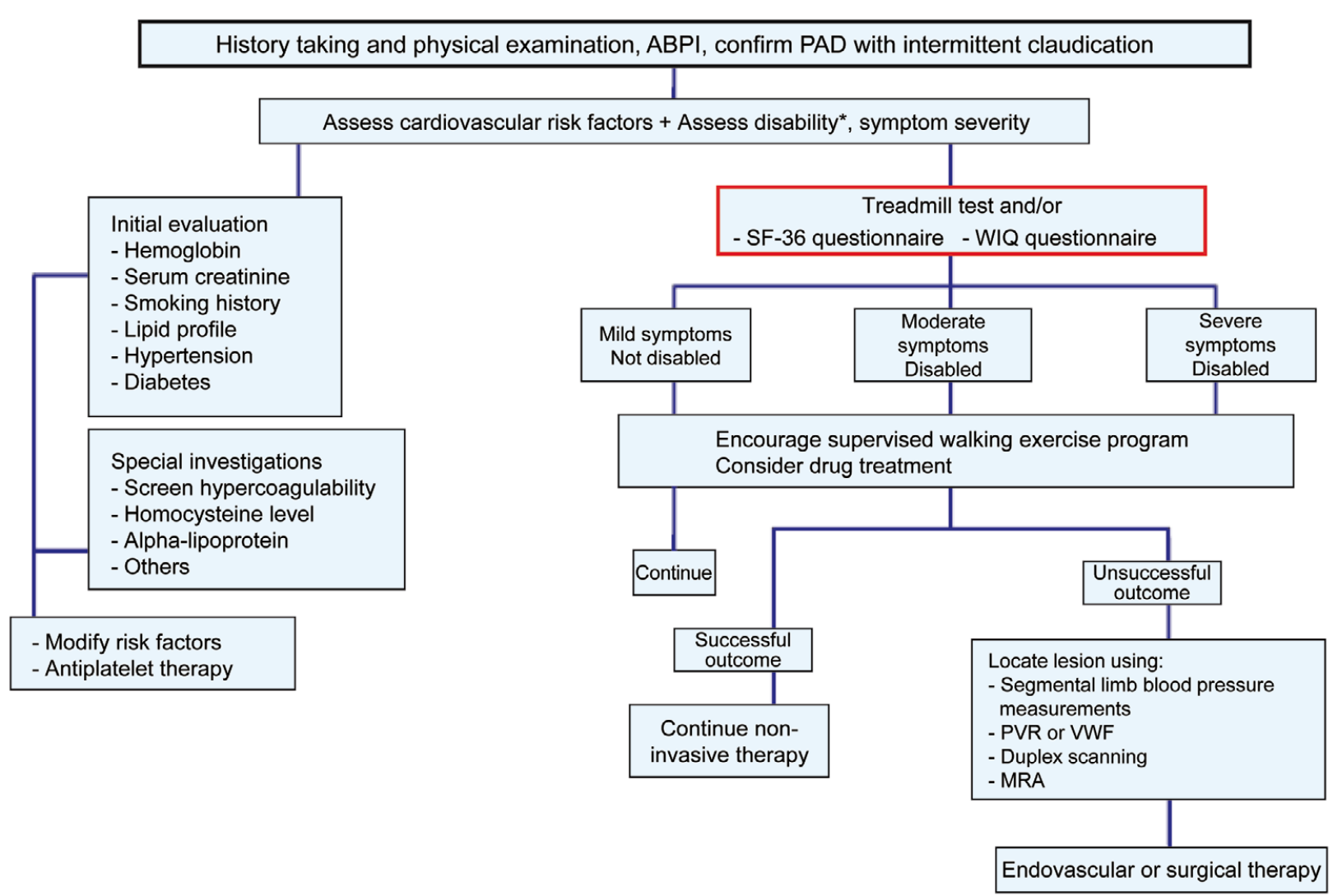

Figure 11. Basic treatment algorism for intermittent claudication (TransAtlantic Inter-Society Consensus: TASC). *Disability as defined by the individual patient. ABPI, ankle-brachial pressure index; MRA, magnetic resonance angiography; PAD, peripheral arterial disease; PVR, pulse volume recording; SF-36, Medical Outcome Study (MOS) 36-Item Short-Form Health Survey; VWF, velocity wave form; WIQ, Walking Impairment Questionnaire. Adapted from TASC Working Group. Management of PAD. J VasC Surg 2000; 31(1 Pt 2): S1-S296, ${ }^{266}$ with permission from Elsevier Inc.

\begin{tabular}{|c|c|}
\hline Method & Supervised exercises are recommended \\
\hline Type & $\begin{array}{l}\text { - Treadmill walking. } \\
\text { - Exercise training programs should consist of (1) warm-up, (2) walking exercise, and (3) cool-down. } \\
\text { - Patients whose major lesions are located in the lower legs, especially those with thromboangitis obliterans may try } \\
\text { Börger's exercise and Ratschow's exercise of which the load to muscles affected by peripheral } \\
\text { ischemia can be modified. } \\
\text { - A recent study has reported that dynamic arm exercise training can improve walking capability in patient with } \\
\text { claudication. This may become a component of exercise programs. }\end{array}$ \\
\hline Intensity & $\begin{array}{l}\text { - Walking exercise should start at grade of } 12 \% \text { and a speed of } 2.4 \mathrm{~km} / \mathrm{h} \text {. The patient should walk at a New Borg scale of } \\
6 \sim 8 / 10 \text { until he/she experiences leg pain (note that the New Borg scale [scale from } 1 \text { to } 10 \text { ] is used for the treatment of } \\
\text { chronic peripheral arterial occlusive disease rather than the conventional Borg scale. See Table } 49 \text { ). } \\
\text { - When the patient can walk at the above intensity for } 10 \text { minutes, the exercise should be intensified to a speed of } \\
3.2 \mathrm{~km} / \mathrm{h} \text { or a higher grade. When the patient can walk at the higher speed, the speed may be further increased to } \\
4.8 \mathrm{~km} / \mathrm{h} \text {. }\end{array}$ \\
\hline $\begin{array}{l}\text { Time, interval, and } \\
\text { duration }\end{array}$ & $\begin{array}{l}\text { - One session of walking exercise should last between } 30 \text { to } 60 \text { minutes. } \\
\text { - The patient should perform } 1 \sim 2 \text { sessions per day, at least } 3 \text { days per week (whenever possible } \geq 5 \text { days per week). } \\
\text { - The patient should walk until he/she feels leg pain similar to the previous pain, and then rest until the pain subsides } \\
\text { repeatedly (for about } 1 \sim 5 \text { minutes). } \\
\text { - Walking exercise is usually continued for } 3 \sim 6 \text { months. } \\
\text { - It has been reported that walking exercise should be continued for at least } 2 \text { or } 3 \text { months. }\end{array}$ \\
\hline Monitoring & - The patient must be monitored for heart/pulse rate and blood pressure. ECG monitoring should also be performed. \\
\hline
\end{tabular}




\begin{tabular}{|c|c|c|c|}
\hline \multicolumn{2}{|c|}{ Borg scale } & \multicolumn{2}{|c|}{ New Borg scale } \\
\hline Scale & RPE & Scale & RPE \\
\hline 20 & Maximum exertion & 0 & Nothing at all \\
\hline 19 & Very, very hard & 0.5 & Very, very weak \\
\hline 18 & & 1 & Very weak \\
\hline 17 & Very hard & 2 & Weak \\
\hline 16 & & 3 & Moderate \\
\hline 15 & Hard & 4 & Somewhat strong \\
\hline 14 & & 5 & Strong \\
\hline 13 & Somewhat hard & 6 & \\
\hline 12 & & 7 & Very strong \\
\hline 11 & Fairly light & 8 & \\
\hline 10 & & 9 & Very, very strong \\
\hline 9 & Very light & 10 & Maximum exertion \\
\hline \multicolumn{4}{|l|}{8} \\
\hline 7 & Very, very light & & \\
\hline 6 & & & \\
\hline
\end{tabular}

Adapted from Borg GA. Psychophysical bases of perceived exertion. Med Sci Sports Exerc 1982; 14: 377-381, ${ }^{268}$ with permission from Wolters Kluwer Health.

\section{Table 50. How to Motivate Patients to Exercise}

1. Interview the patient in detail to obtain information necessary to provide practical advice to modify lifestyle.

2. The instructor should walk with the patient whenever possible to make exercise more engaging.

3. Discuss specific feasible exercise prescriptions with the patient, and try prescriptions (talk with the patient with a positive attitude such as "Let's try. If you fail, try again").

4. Instruct the patient to keep an exercise log and record pedometer counts (as same as measurements of blood pressure and blood glucose). Encourage family members to support the patient.

1) Set specific numerical goals (self-validation)

2) Instruct the patient to remind the goals every day (repetition)

3) Encourage family members to remind the patient of the goals (repetition)

Source: Yasu T, et al. Exercise training for vascular diseases. Journal of the Japanese Association of Cardiac Rehabilitation (JJCR) 2008; 1 $39-42 .{ }^{269}$

\section{(2) Exercise Prescriptions (Tables 48 and 49268)}

\section{(3) How to Motivate Patients Participating in Exercise Programs (Table 50) 269}

It has been reported that about $50 \%$ of patients who have been able to continue exercise training for 2 weeks can continue exercise training thereafter.

Patients undergoing exercise training should be supported and followed up by healthcare professionals and staff members of cardiac rehabilitation programs. ${ }^{269}$ Multidisciplinary patient education is also important to develop a patient's better understanding of prescribed exercise training.

\section{(4) Home-Based Exercise Training}

Patients should walk fast with a pedometer at a New Borg scale of 6 8/10 ("somewhat strong"). Patients should be instructed to perform 30-minute walking exercise sessions twice a day, 5 days a week. One session should consist of sequences of walking a little faster than usual until pain develops and a several-minute rest until pain disappears.
5. Exercise Training and Life Style Management to Control Risk Factors for Atherosclerosis

(1) Exercise training

(2) Risk factor management: Goals include smoking cessation, LDL cholesterol $<100 \mathrm{mg} / \mathrm{mL}$, hemoglobin A1c (HbA1c) (NGSP [National Glycohemoglobin Standardization Program] $<7.0 \%$, and blood pressure $<140 / 90 \mathrm{mmHg}$.

(3) Use of antiplatelet drugs to prevent cerebrovascular and cardiovascular diseases. ${ }^{258,270}$

(4) Use of $\beta$-blockers in patients after AMI to improve prognosis. $^{271}$

\section{Combination of Exercise Training and Drug Treatment} in Patients With Intermittent Claudication

Exercise training is the first choice treatment for ischemic claudication. ${ }^{272,273}$ Drug treatment is also effective. ${ }^{274-276}$ (Level of

\section{Evidence: C)}

The combination of drug treatment and exercise training is also recommended for patients with ischemic claudication. ${ }^{277-280}$

A combination of heparin therapy (administer 3,000 IU 60 minutes before exercise training) and exercise training for 14 days is also effective. ${ }^{281}$ 
VIII Psychological Approaches for the Rehabilitation in Patients With Cardiovascular Diseases

\section{Class I}

None.

\section{Class Ila'}

1. Because the prevalence of depression and depressed state is high among patients with CAD, and depression has been reported to relate to increased morbidity and mortality from cardiovascular diseases, screening for and treatment of depression by specialists are useful for this patient population. (Level of Evidence: B)

\section{Class Ilb}

1. Screening of depressed mood or other psychological symptoms as well as psychological intervention for the prevention and treatment of depression are expected to alleviate psychological symptoms, improve QOL, enhance adherence to treatment, and reduce the mortality and incidence of cardiovascular events. (Level of Evidence: C)

2. Therapeutic interventions for patients with certain psychosocial characteristics such as Type D (distressed) personality are expected to reduce the mortality and incidence of cardiovascular events. (Level of Evidence: C)

\section{Importance of Clinical Psychological Interventions for Patients With Cardiovascular Diseases}

Patients with cardiovascular diseases often experience psychological symptoms such as depression and anxiety disorders that are known to significantly affect life expectancy and prognosis by increasing mortality and the incidence of cardiovas- cular events in this patient population. ${ }^{282-289}$ It also has been reported that psychological stress associated with physical stress, anxiety and depression, and Type D personality may predict the health status of patients with cardiac disease. ${ }^{284,290-292}$

The prevalence of depression among patients with AMI is about 3 times higher than that seen in the general population, and $15 \sim 20 \%$ of patients with AMI have a major depressive disorder (MDD) ${ }^{293,294}$ as defined in the DSM-IV-TR (Diagnostic and Statistical Manual of Mental Disorders, 4th edition, text revision) (Table 51). ${ }^{295,296}$ Similar prevalence of MDD have been reported among patients with unstable angina, those after PCI, CABG and valve surgery, and a higher prevalence of MDD has been reported in patients with chronic heart failure. ${ }^{297,298}$ Depression not only substantially deteriorates QOL in patients with cardiac disease, ${ }^{299,300}$ but also decreases adherence to drug treatment, ${ }^{301}$ leads poor lifestyle choices such as decreased physical activity and increased smoking that may increase the risk of cardiovascular disease. And reduce compliance with treatment and lifestyle intervention plans that are essential to modify cardiovascular risk factors, ${ }^{302}$ attendance at cardiac rehabilitation programs, ${ }^{303,304}$ and increases healthcare costs. ${ }^{282,305}$ It has recently been advocated that screening and intervention for depression are essential for patients with cardiovascular diseases.

Reports have described that psychological factors such as anger, Type D personality, and psychological stress associated with physical stress, anxiety, and depression, may predict health

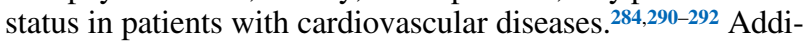
tionally, Type D personality is reported to be more strongly related to cardiac mortality and incidence of cardiac events than other factors such as decreased LVEF, impaired exercise capacity, and the presence/absence of three-vessel disease.

\section{Table 51. Criteria for Major Depressive Episode}

A. At least five of the following symptoms have been present during the same 2-week period and represent a change from previous functioning; at least one of the symptoms is either (1) depressed mood or (2) loss of interest or pleasure.

1. Depressed mood most of the day, nearly every day, as indicated by either subjective report (e.g., feels sad or empty) or observation made by others (e.g., appears tearful).

2. Markedly diminished interest or pleasure in all, or almost all, activities most of the day, nearly every day (as indicated by either subjective account or observation made by others).

3. Significant weight loss when not dieting or weight gain (e.g., a change of more than $5 \%$ of body weight in a month), or decrease or increase in appetite nearly every day.

4. Insomnia or hypersomnia nearly every day.

5. Psychomotor agitation or retardation nearly every day (observable by others, not merely subjective feelings of restlessness or being slowed down).

6. Fatigue or loss of energy nearly every day.

7. Feelings of worthlessness or excessive or inappropriate guilt (which may be delusional) nearly every day (not merely self-reproach or guilt about being sick).

8. Diminished ability to think or concentrate, or indecisiveness, nearly every day (either by subjective account or as observed by others).

9. Recurrent thoughts of death (not just fear of dying), recurrent suicidal ideation without a specific plan, or a suicide attempt or a specific plan for committing suicide.

B. The symptoms do not meet criteria for a mixed episode.

C. The symptoms cause clinically significant distress or impairment in social, occupational, or other important areas of functioning.

D. The symptoms are not due to the direct physiological effects of a substance (e.g., a drug of abuse, a medication) or a general medical condition (e.g., hypothyroidism).

E. The symptoms are not better accounted for by bereavement, i.e., after the loss of a loved one, the symptoms persist for longer than 2 months or are characterized by marked functional impairment, morbid preoccupation with worthlessness, suicidal ideation, psychotic symptoms, or psychomotor retardation.

Source: American Psychiatric Association. Diagnostic and Statistical Manual of Mental Disorders, 4th edn, Text Revision (DSM-IV-TR). Washington, DC: American Psychiatric Association, 2000. ${ }^{295}$ 
Table 52. Psychological, Mental and QOL Scales Commonly Used for Patients With Cardiovascular Diseases

\begin{tabular}{|c|c|c|}
\hline Evaluation & Title of questionnaire & Number of questions/contents \\
\hline Anxiety & STAI: State-Trait Anxiety Inventory & 40 questions. A measure of state anxiety and trait anxiety. \\
\hline Depression & PHQ-9: Patient Health Questionnaire-9 & $\begin{array}{l}10 \text { questions. A measure used as a screening tool for } \\
\text { depression (measure of depressive state). }{ }^{*} \text { An application for } \\
\text { research use may be required. }\end{array}$ \\
\hline Depression & $\begin{array}{l}\text { BDI-II: Beck Depression Inventory-Second } \\
\text { Edition }\end{array}$ & $\begin{array}{l}21 \text { questions. Measure depressions over the previous } 2 \\
\text { weeks (weight on mood and cognition). }\end{array}$ \\
\hline Depression & SDS: Self-rating Depression Scale & $\begin{array}{l}20 \text { questions. Self-rating depression scale (severity of } \\
\text { depression and efficacy evaluation). }\end{array}$ \\
\hline Depression & $\begin{array}{l}\text { CES-D: Center for Epidemiologic Studies } \\
\text { Depression Scale }\end{array}$ & $\begin{array}{l}20 \text { questions. Self-rating scale for depression (depressive } \\
\text { state). }\end{array}$ \\
\hline Depression & $\begin{array}{l}\text { HDRS: Hamilton Rating Scale for Depression } \\
\text { (HAM-D) }\end{array}$ & $\begin{array}{l}17 \text { questions ( } 21 \text { or } 24 \text { questions in some language } \\
\text { versions). An interview scale used by specialists. }\end{array}$ \\
\hline Depression/anxiety & HADS: Hospital Anxiety and Depression Scale & $\begin{array}{l}14 \text { questions. A scale for evaluation of depression and } \\
\text { anxiety in patients with physical diseases. }\end{array}$ \\
\hline Emotion/mood & POMS: Profile of Mood States & $\begin{array}{l}65 \text { questions. A scale to assess transient, fluctuating active } \\
\text { mood states. }\end{array}$ \\
\hline Type D personality & DS14: Type D Scale-14 & $\begin{array}{l}14 \text { questions. A Type } D \text { (distressed) personality scale based } \\
\text { on negative affectivity and social inhibition. }\end{array}$ \\
\hline Anger & $\begin{array}{l}\text { STAXI-2: State-Trait Anger expression } \\
\text { Inventory-2 (Upgrade version of STAXI) }\end{array}$ & $\begin{array}{l}57 \text { questions. Developed to assess state anger, trait anger, } \\
\text { and anger expression. }\end{array}$ \\
\hline Personality inventory & $\begin{array}{l}\text { MMPI: Minnesota Multiphasic Personality } \\
\text { Inventory }\end{array}$ & $\begin{array}{l}550 \text { questions. An international questionnaire that evaluates } \\
\text { personality traits multilaterally and is translated into } 130 \\
\text { languages. }\end{array}$ \\
\hline Mental health & GHQ: General Health Questionnaire & $\begin{array}{l}60 \text { questions ( }{ }^{*} \text { Shorter versions consist of } 30 / 28 / 12 \\
\text { questions). A scale to diagnose and evaluate the severity of } \\
\text { mental disorders (mental health). }\end{array}$ \\
\hline QOL & $\begin{array}{l}\text { SF-36: The 36-item short form of the Medical } \\
\text { Outcomes Study Questionnaire }\end{array}$ & $\begin{array}{l}36 \text { questions. A scale of health-related QOL including } \\
\text { physical function and role-emotional limitation. }\end{array}$ \\
\hline QOL & WHO/QOL-26: WHO/Quality of Life-26 & $\begin{array}{l}26 \text { questions. A measure of QOL consisting of physical } \\
\text { domain, psychological domain, social relationships, } \\
\text { environment, and } 2 \text { items for general QOL. }\end{array}$ \\
\hline
\end{tabular}

This possible correlation is receiving increasing attention..$^{306}$

\section{Psychological Assessment of Patients With Cardiovascular Diseases}

Psychological assessment should include (1) careful consideration of the patient's physical/mental-health status to select suitable methods for assessment, (2) appropriate feedback of the results of assessment, and (3) satisfactorily explained to all staffs with thoughtful word to share necessary information. The third point is most important in patients who have prejudice or resistance against mental health issues may deny their symptoms or have a feeling of anger or resistance when psychological assessment is suggested. Physicians and staff members should fully explain about the importance of psychological assessment and provide adequate support to patients. ${ }^{302}$ Table 52 lists psychological, mental and QOL measures that are commonly used for rehabilitation in patients with cardiovascular diseases.

\section{Psychological Interventions for Patients With Cardiovascular Diseases}

The most common symptoms that require psychological interventions among patients with cardiovascular disease are depressive and anxious state. It has been reported that health outcomes are poorer among patients who have depression and a chronic physical disorder than for patients who have two or more chronic physical disorders. ${ }^{307}$ The basic components of depression treatment are drug treatment and psychological therapy. Although the efficacy of antidepressants has been established most convincingly in the treatment of MDD, it has been pointed out that the efficacy depends on the severity of depression. ${ }^{308}$ And more antidepressants are not recommended as first-line therapy for patients with mild depression, because they do not respond to drug treatment as effectively as those in severe depression..$^{309}$ Antidepressants are not indicated as firstline therapy for patients with mild depression, who have been reported to respond well to exercise and psychological therapy. Physicians should consider all available treatment options, and not limit their choices to antidepressants. ${ }^{310}$

\section{Drug Treatment}

Depression and depressive states are commonly observed in patients with cardiovascular diseases. ${ }^{298}$ Antidepressants currently available in Japan include tricyclic antidepressants, tetracyclic antidepressants, selective serotonin reuptake inhibitors (SSRIs), serotonin and norepinephrine reuptake inhibitors (SNRIs), and noradrenergic and specific serotonergic antidepressants (NaSSAs). The AHA recommends sertraline* and citalopram** (both are SSRIs) as first-line therapy for patients with CAD and depression. ${ }^{311}$ These two SSRIs have been demonstrated in many clinical studies to be safe in patients with cardiovascular diseases and effective in the treatment of moderate or severe depression and recurrent depression. If the patient expresses suicidal ideation, has strong anxiety or irritation, or has no improvement after 2 months of treatment, the patient should be referred to a psychiatrist to provide multidis- 
[Support for all participants]

Providing information

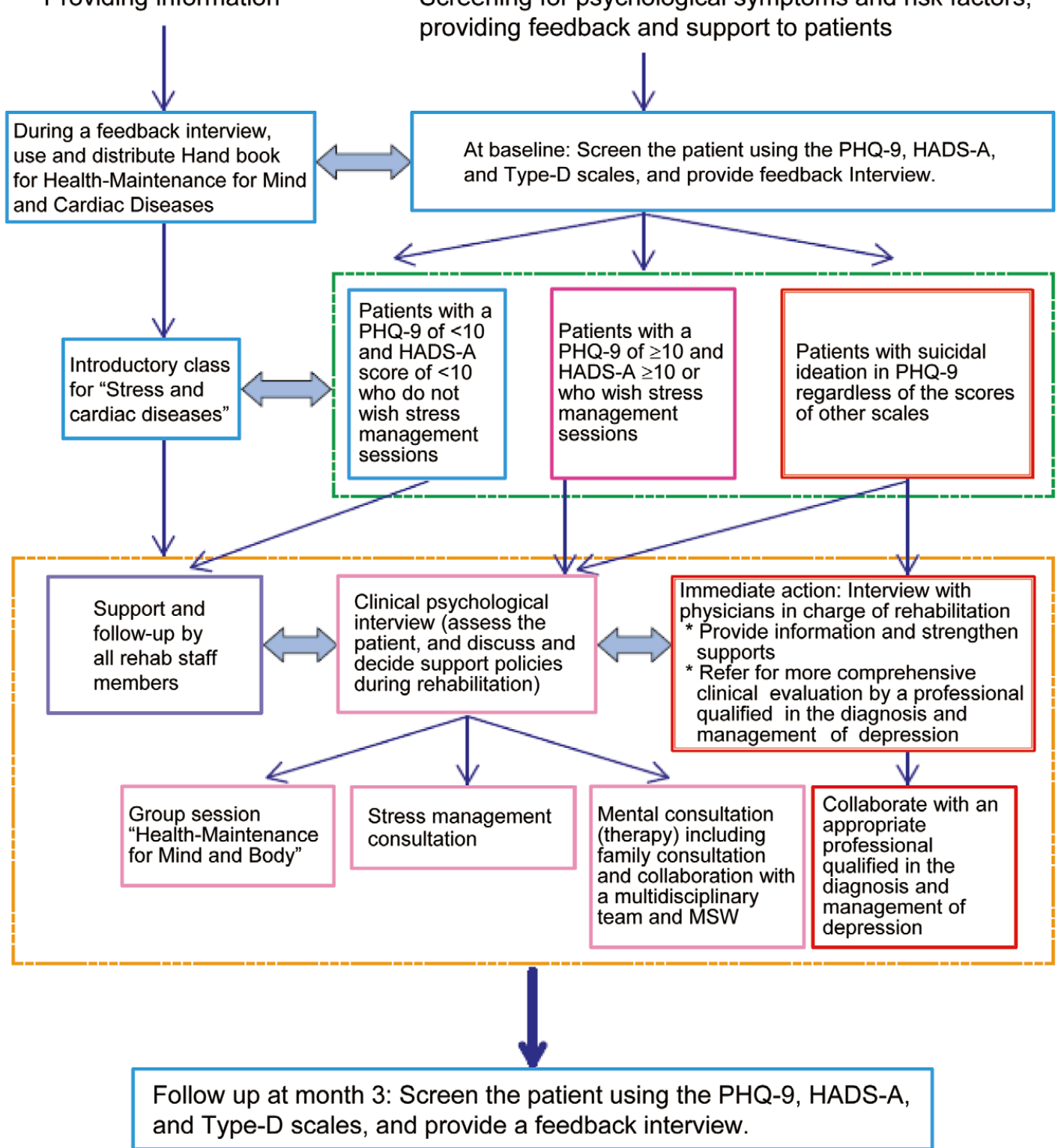

Figure 12. An example of screening for psychological symptoms and support during cardiac rehabilitation (A System Used in Sakakibara Heart Institute). *HADS-A, anxiety subscale of the Hospital Anxiety and Depression Scale; MSW, medical social workers; PHQ-9, Patient Health Questionnaire-9.

ciplinary treatment. Antidepressant treatment is usually continued as maintenance therapy for months or 1 year after symptoms of depression are controlled, and treatment should be tapered off gradually with careful monitoring for recurrence.

\section{[Precaution Statement]}

*Sertraline:

MEDSAFE (Medicines and Medical Devices Safety Authority) of New Zealand decided that Sertraline hydrochloride needs the similar precautions as citalopram hydrobro- mide on December 2012. And Pfizer revised their CCDS (Company Core Data Sheet for Sertraline hydrochloride in Japan) on September 2013.

**Citalopram:

In August 2013, The U.S. Food and Drug Administration (FDA) is clarifying dosing and warning recommendations for the antidepressant Celexa (citalopram hydrobromide) below. Citalopram is not recommended for use in patients with congenital long QT syndrome, bradycardia, hypokalemia, or hypomagnesemia, recent AMI, or uncompensated 
heart failure.

\section{Psychological Therapy}

Psychological supports to patients with cardiovascular diseases are classified into (1) psychological patient education to assist patients to acquire skills in coping with mental stresses, (2) psychological therapy provided to alleviate depressed mood and anxiety or solve personal problems, (3) psychological interview to reduce psychological risks on diseases that provided as a component of comprehensive cardiac rehabilitation programs.

The efficacy of psychological therapy in patients with cardiovascular diseases has been investigated in studies using several methodologies such as interpersonal psychotherapy, ${ }^{312}$ cognitive behavioral therapy, ${ }^{310,313}$ and problem-solving therapy. ${ }^{314,315}$ Autogenic training, a relaxation technique to relieve insomnia and anxiety, is also used in this patient population. ${ }^{316}$

\section{Other Possibly Effective Approaches}

Exercise training and aerobic exercise training conducted as a part of cardiac rehabilitation are effective in improving cardiovascular function, reducing mortality due to cardiovascular diseases, alleviating depressive state, ${ }^{317-319}$ and reducing psychosocial stress. ${ }^{320,321}$ Social supports help to alleviate psychological symptoms such as depression in patients with cardiovascular diseases, ${ }^{322-324}$ and should also cover family members who care for the patient. ${ }^{302}$

\section{Psychological Interventions to Cardiac Rehabilitation}

In addition to providing psychological interventions, psychologists may be involved in cardiac rehabilitation by (1) screening for mental disorders and providing appropriate feedback to cardiologists, (2) providing patient education (providing information) on the relationship between cardiovascular diseases and psychological symptoms such as depression, (3) alleviating psychological symptoms such as depressed mood and anxiety, (4) assisting patients to acquire skills in coping with mental stresses, (5) helping patients rebuild self-efficacy and self-confidence, maintain social relationships, prevent social isolation, and enhance social supports. ${ }^{304}$

Because continuous multidisciplinary supportive care is effective in alleviating symptoms of depression and suicidal ideation, ${ }^{327}$ psychologists should share their insights with other staff members involved in cardiac rehabilitation, and ensure the development of an efficient, appropriate multidisciplinary cardiac rehabilitation system.

Patients should be regularly screened for mental disorders and be monitored continuously because the presence/absence and severity of depressed mood and anxiety are affected by daily events and mental stress. In addition, since patients do not always exhibit more severe depressed mood or anxiety symptoms immediately after the onset of cardiovascular events (Figure 12).

\section{Building an Exercise Training System}

\section{Approaches to Exercise Training Programs: How to Build Systems}

\section{A Prospective View of Cardiac Rehabilitation in Japan \\ (1) Efficient Use of Hospitals Providing Cardiac Rehabilitation Programs}

\section{Class I}

1. It is recommended that patients participate in recoveryphase cardiac rehabilitation programs. (Level of Evidence: A)

\section{Class IIa}

1. Cardiac rehabilitation only during hospitalization. (Level of Evidence: C)

Patients should continue cardiac rehabilitation programs for life to prevent recurrent cardiac diseases and improve cardiac function. As the duration of hospitalization is becoming shorter, patients with cardiovascular diseases should participate in outpatient cardiac rehabilitation programs after discharge and utilize cardiac rehabilitation facilities that have the capacity to provide services to out-patients.

\section{(2) Comprehensive Cardiac Rehabilitation Programs (Table 53)}

\section{Class I}

1. It is recommended that cardiac rehabilitation programs include patient education sessions. (Level of Evidence: A)

It has been demonstrated that comprehensive cardiac rehabilitation programs that include patient education sessions re- duce mortality by $73 \%$ as compared with programs consisting of exercise sessions only. It is desirable that cardiac rehabilitation programs should include not only exercise training, but also diet therapy, smoking cessation counseling, stress management, and lifestyle intervention.

\section{Functions Required for Cardiac Rehabilitation Class I}

1. It is recommended that cardiac rehabilitation involve a multidisciplinary team including nurses, physical therapists and other exercise trainer. (Level of Evidence: C)

Cardiac rehabilitation programs must include exercise training, diet therapy, and lifestyle intervention, and should involve a multidisciplinary team including physical therapists, occupational therapists, health fitness programmers, dieticians and nurses. Clinical psychotherapists, medical technologists, and pharmacists should also be involved whenever necessary. Certified cardiac rehabilitation specialists should lead cardiac rehabilitation programs.

\section{Roles of Nurses in Comprehensive Cardiac Rehabilitation \\ Class I}

1. Patient education programs led by nurses are recommended as these programs are effective in modifying coronary risk factors. (Level of Evidence: A)

It has been reported that nurse-led management programs significantly decrease the morbidity and mortality of CAD. ${ }^{328-334}$

There is an urgent need to foster specialists who manage cardiac rehabilitation programs, and nurses are expected to 


\begin{tabular}{|c|c|}
\hline Item & Description \\
\hline \multicolumn{2}{|l|}{ Duration } \\
\hline Outpatient & 1 hour/session (3 sessions) \\
\hline Inpatient & $\geq 1$ hour/day \\
\hline Supervision & $\begin{array}{l}\text { A physician should supervise patients (or should be available at all times to } \\
\text { healthcare professionals who are attending the session), and describe the } \\
\text { progress of rehabilitation in patients' medical records. }\end{array}$ \\
\hline \multicolumn{2}{|l|}{ No. of patients } \\
\hline Inpatient & $\begin{array}{l}\text { The number of patients supervised by a physician during a session is about } 15 \text {, } \\
\text { and that attended by a physical therapist or nurse is about } 5 \text {. }\end{array}$ \\
\hline Outpatient & The corresponding numbers are about 20 and 8. \\
\hline No. of sessions provided & $\begin{array}{l}\text { A healthcare professional excluding physician may provide } \leq 18 \text { sessions a day } \\
\text { and } \leq 108 \text { sessions a week. }\end{array}$ \\
\hline Calculation of $\mathrm{NHI}$ reimbursement & $\begin{array}{l}\text { Fees for ECG, exercise ECG, and respiratory/pulse rate monitoring should be } \\
\text { included in the fee for cardiac rehabilitation. }\end{array}$ \\
\hline \multirow[t]{3}{*}{ Documents required } & $\begin{array}{l}\text { - An integrated protocol* of "cardiovascular rehabilitation" (prepared once a } \\
\text { month). Explain the progress of rehabilitation using the protocol* (at beginning } \\
\text { and every } 3 \text { months thereafter). }\end{array}$ \\
\hline & $\begin{array}{l}\text { - Documents about rehabilitation (physician's instructions, exercise } \\
\text { prescriptions, durations, contents of session, and supervisors), and exercise } \\
\text { logs stamped by physicians, physical therapists, and nurses should be kept in } \\
\text { the same file. }\end{array}$ \\
\hline & $\begin{array}{l}\text { - Multidisciplinary team should have conferences periodically to discuss the } \\
\text { individual program. }\end{array}$ \\
\hline
\end{tabular}

${ }^{*}$ Refer to Tables $54^{325}$ and $55^{325}$ for samples of rehabilitation protocols, and Table $56^{326}$ for a sample of an integrated protocol.

$\mathrm{NHI}$, National Health Insurance.

fulfil the role of cardiac rehabilitation instructors.

\section{Cardiac Rehabilitation Programs and Disease Management After Discharge From Hospital}

\section{Class I}

1. After discharge from hospital, it is recommended that all patients participate in cardiac rehabilitation programs to prevent recurrent cardiovascular events and improve prognosis. (Level of Evidence: A)

2. Maintenance-phase cardiac rehabilitation programs are effective in preventing recurrent cardiovascular events and improving QOL and prognosis, and are recommended for patients with cardiovascular diseases. (Level of Evidence: A)

\section{Class Ila}

1. It is appropriate that maintenance-phase comprehensive cardiac rehabilitation be continued for life. (Level of Evidence: B)

2. It is appropriate that disease management be continued for life. (Level of Evidence: C)

\section{Class IIb}

1. Cardiac rehabilitation only during hospitalization should be considered. (Level of Evidence: B)

2. Community-based cardiac rehabilitation programs are effective as maintenance-phase cardiac rehabilitation programs. (Level of Evidence: B)

3. Inappropriate work-rest balance and sleep disorders increase the risk of cardiovascular diseases. (Level of Evidence: B)

Cardiac rehabilitation programs after discharge from hospital consist of late phase II (outpatient recovery phase) and phase
III (maintenance phase) cardiac rehabilitation programs. Maintenance-phase cardiac rehabilitation programs serve as measures of secondary prevention of recurrent cardiovascular events, and of primary prevention of atherosclerotic diseases.

\section{Late Phase II (Late Recovery Phase) Cardiac Rehabilitation}

Late phase II cardiac rehabilitation programs are provided as ambulatory programs after discharge. As patients with cardiovascular diseases typically visit the clinic biweekly during the first 1 2 months after discharge for follow-up during this period, they should participate in comprehensive cardiac rehabilitation programs in which outpatient nurses play a central role in educating patients on smoking cessation, diet, and lifestyle interventions. In addition to education, patients should continue exercise training mainly consisting of endurance training, and undergo exercise stress tests at months 1 and 3 and at the end of the program to assess the efficacy of rehabilitation programs, predict prognosis, and reissue exercise prescriptions.

\section{Phase III (Maintenance Phase) Cardiac Rehabilitation}

Maintenance phase cardiac rehabilitation should be continued for life to prevent recurrent cardiovascular events. Cardiac rehabilitation becomes more effective as it continues, and it has been demonstrated that long-term cardiac rehabilitation programs significantly decrease total mortality and the rate of cardiovascular deaths. ${ }^{5}$

Exercise at the AT level is recommended in this phase. However, as patients in this phase have a broader margin of safety, the exercise intensity in the maintenance-phase rehabilitation programs may be set according to the maximum heart rate on maximal exercise stress tests using the Karvonen Formula. 


\section{Table 54. Rehabilitation Protocol}

(Attached Form 21-4)

Rehabilitation Protocol

\begin{tabular}{|c|c|c|c|c|c|c|}
\hline \multirow{2}{*}{ ID: } & \multirow{2}{*}{\multicolumn{2}{|c|}{$\mathrm{M} / \mathrm{F}$}} & \multicolumn{4}{|c|}{ Date of assessment (Start date): } \\
\hline & & & \multirow{2}{*}{\multicolumn{3}{|c|}{ Rehab physician: }} & years age) \\
\hline \multicolumn{3}{|c|}{ Attending physician/Explained by: } & & & & Rehab nurse: \\
\hline \multicolumn{3}{|c|}{ Physical therapist: } & \multicolumn{4}{|c|}{ Other professionals ( $\quad$ ) involved: } \\
\hline Diagnosis & & & & \multicolumn{2}{|c|}{ Complications } & \\
\hline Hospitalized on: & & & & \multicolumn{2}{|c|}{ Date of onset (approx.): } & \\
\hline Date of surgery: & & & & \multicolumn{2}{|c|}{ Treatment (surgical) procedures } & \\
\hline $\begin{array}{l}\text { Coronary risk } \\
\text { factors (past illness) }\end{array}$ & \multicolumn{6}{|c|}{$\begin{array}{l}\square \text { Hypertension } \square \text { Dyslipidemia } \square \text { Diabetes } \square \text { Hyperuricemia } \square \text { Chronic kidney disease (CKD) } \\
\square \text { Obesity } \square \text { Smoking } \quad \square \text { Old myocardial infarction } \square \text { Angina pectoris } \square \text { Family history } \square \text { Others: }\end{array}$} \\
\hline \multirow[t]{3}{*}{$\begin{array}{l}\text { Risk during } \\
\text { exercise }\end{array}$} & Cardiac function & $\begin{array}{l}\square \text { Normal } \\
\square \text { Reduce }\end{array}$ & $\mathrm{d}(\mathrm{EF}$ & $\%)$ & $\begin{array}{l}\text { Limited range of } \\
\text { joint motion }\end{array}$ & \begin{tabular}{|l}
$\square$ Absent \\
$\square$ Present (
\end{tabular} \\
\hline & Arrhythmias & $\begin{array}{l}\square \text { Absent } \\
\square \text { Present }\end{array}$ & & ) & $\begin{array}{l}\text { Ischemia } \\
\text { (residual stenosis) }\end{array}$ & \begin{tabular}{|l}
$\square$ Absent \\
$\square$ Present (
\end{tabular} \\
\hline & Others & & & & & \\
\hline \multicolumn{7}{|c|}{ Activities in the ward (currently conducted activities) } \\
\hline Movement & \multicolumn{6}{|c|}{\begin{tabular}{|l}
$\square$ On the bed $\square$ Using a wheelchair $\square$ Walking in the room $\square$ Walking in the ward \\
$\square$ Walking outdoors
\end{tabular}} \\
\hline $\begin{array}{l}\text { Turning and } \\
\text { positioning in bed }\end{array}$ & \multicolumn{4}{|c|}{$\square$ Independent $\square$ Supervised $\square$ Supported } & \multicolumn{2}{|l|}{${ }^{*}$ Remarks: } \\
\hline $\begin{array}{l}\text { Walking } \\
\text { (transferring) }\end{array}$ & \multicolumn{4}{|c|}{\begin{tabular}{|l}
$\square$ Independent $\square$ Supervised $\square$ Supported \\
$\square$ Not performed yet
\end{tabular}} & $\begin{aligned} &{ }^{*} \text { Remarks: } \square \text { Indep } \\
& \square \text { Stretcl } \\
&\end{aligned}$ & $\begin{array}{l}\square \text { Independently } \\
\square \text { Stretcher }\end{array}$ \\
\hline $\begin{array}{l}\text { Transfer from bed } \\
\text { to wheelchair } \\
\text { (stretcher) }\end{array}$ & \multicolumn{4}{|c|}{\begin{tabular}{|l}
$\square$ Independent $\square$ Supervised $\square$ Supported \\
$\square$ Not performed yet
\end{tabular}} & \multicolumn{2}{|l|}{${ }^{*}$ Remarks: } \\
\hline Meals & \multicolumn{4}{|c|}{$\begin{array}{l}\square \text { Independent } \square \text { Supervised } \square \text { Supported } \\
\square \text { Not performed yet }\end{array}$} & \multicolumn{2}{|l|}{${ }^{\star}$ Remarks: } \\
\hline Dressing & \multicolumn{4}{|c|}{$\square$ Independent $\square$ Supervised $\square$ Supported } & \multicolumn{2}{|c|}{ "Remarks: $\square$ IV line $\square$ No IV line $\square$ Others: } \\
\hline Urination & \multicolumn{4}{|c|}{$\square$ Independent $\square$ Supervised $\square$ Supported } & \multicolumn{2}{|c|}{$\begin{aligned}{ }^{*} \text { Remarks: } & \square \text { Toilet for wheelchair } \square \text { Portable toilet } \\
& \square \text { Chamber pot } \square \text { Urinary catheter } \\
& \square \text { Others: }\end{aligned}$} \\
\hline Defecation & \multicolumn{4}{|c|}{$\square$ Independent $\square$ Supervised $\square$ Supported } & \multicolumn{2}{|c|}{$\begin{array}{ll}{ }^{*} \text { Remarks: } & \square \text { Toilet for wheelchair } \quad \square \text { Portable toilet } \\
& \square \text { On the bed }\end{array}$} \\
\hline Hygiene & \multicolumn{4}{|c|}{$\square$ Independent $\square$ Supervised $\square$ Supported } & \multicolumn{2}{|c|}{ * Remarks: $\square$ Bath $\square$ Shower $\square$ Shampoo $\square$ Dry bath } \\
\hline Communication & \multicolumn{6}{|c|}{$\square$ Communicable $\square$ Somewhat difficult $\quad \square$ Difficult $\quad{ }^{*}$ Remarks: } \\
\hline Housing & \multicolumn{4}{|c|}{$\begin{array}{l}\square \text { Living alone } \square \text { Living with others ( ) } \\
\square \text { House } \square \text { Apartment }\end{array}$} & Living on the th $\mathrm{fl}$ & oor $\square$ with or $\square$ without elevator \\
\hline Occupation & \begin{tabular}{|l|} 
Unemployed $\square$ \\
$\square$ Physical worker
\end{tabular} & Houseperson & $\square$ Offic & e worker & $\begin{array}{l}\text { Job type/how to } \\
\text { commute }\end{array}$ & \\
\hline $\begin{array}{l}\text { Goals to prevent } \\
\text { recurrence and } \\
\text { maintain health }\end{array}$ & $\begin{array}{l}\square \text { Understand the d } \\
\square \text { Improve physical }\end{array}$ & $\begin{array}{l}\text { lisease } \square \mathrm{Cc} \\
\text { strength } \square \text { ? }\end{array}$ & $\begin{array}{l}\text { Omplianc } \\
\text { Stop sm }\end{array}$ & $\begin{array}{l}\text { to drug treat } \\
\text { king } \square \text { Othe }\end{array}$ & $\begin{array}{l}\text { Iment } \quad \square \text { Dietary mans } \\
\text { ers: }\end{array}$ & agement $\square$ Build exercise habit \\
\hline $\begin{array}{l}\text { Desire/goals of } \\
\text { patients/family }\end{array}$ & & & & & & \\
\hline
\end{tabular}

\begin{tabular}{|c|c|c|}
\hline Start date (Day 1) & Planned duration & \\
\hline $\begin{array}{l}\text { Goals/policy of } \\
\text { exercise programs } \\
\text { (stress test) }\end{array}$ & $\begin{array}{l}\square \text { Standing and walking around the bed } \square 50 \mathrm{~m} \text { walking } \square 100 \mathrm{~m} \text { walkin } \\
\square \text { ( ) m walking } \square \text { Treadmill stress test } \square \text { Cycle ergometer test } \square 6 \\
\square \text { Others: }\end{array}$ & $\begin{array}{c}\square 200 \mathrm{~m} \text { walking } \square 500 \mathrm{~m} \text { walking } \\
\text { ninute walking test }\end{array}$ \\
\hline $\begin{array}{l}\text { Exercise and } \\
\text { prescription }\end{array}$ & $\begin{array}{l}\text { Respiratory training } \square \text { Stretching } \square \text { Muscle strengthening } \square \text { Activitie } \\
\square \text { Others: }\end{array}$ & of daily living $\square$ Walking $\square$ Cycling \\
\hline
\end{tabular}

I acknowledge that the above information was fully explained.

Date:

Signature of the patient or family member

Source: http://www.mhlw.go.jp/seisakunitsuite/bunya/kenkou_iryou/iryouhoken/iryouhoken15/dl/2-22-5.pdf. ${ }^{225}$

\section{Disease Management}

Disease management programs for patients with cardiovascular diseases are provided to help patients modify their lifestyles for better health for life through comprehensive programs. Such programs should be supported by family members, communities, and framework beyond the bounds of medical care 


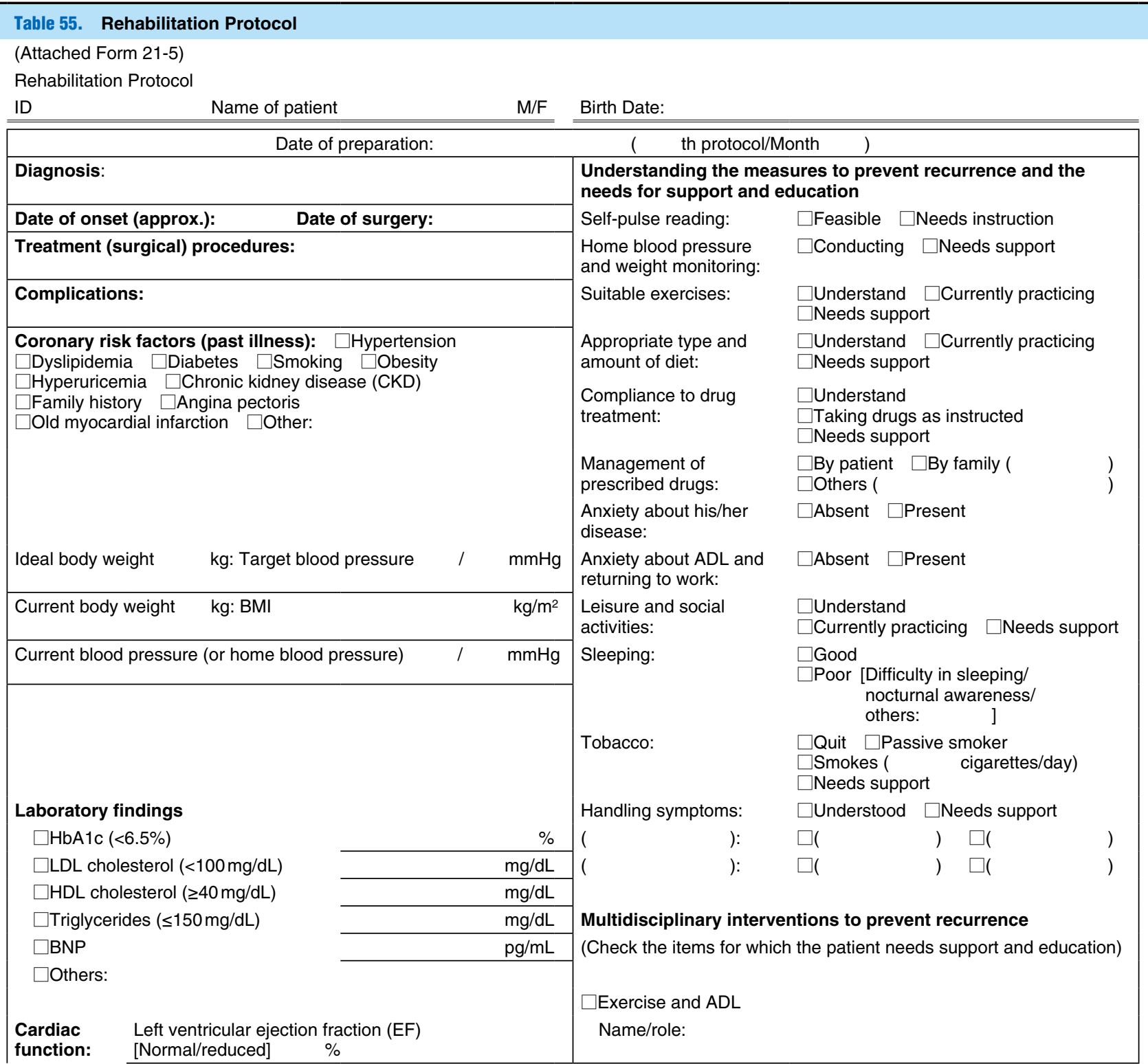

(Table 55 continued the next page.)

to encourage patients to keep the necessary motivation to modify their lifestyle and behavior, maintain health, and ensure secondary prevention of recurrent cardiovascular events and primary prevention of new conditions.

To ensure successful long-term support, approaches to enhance the motivation of the participants, the continuous education by healthcare professionals, and the social support by communities are important. ${ }^{335,336}$ Specifically, there should be systems to provide individualized programs led by specialists with experience and expertise, useful indexes of exercise intensity and quantity, and non-hospital facilities for continued exercise training programs in communities.

\section{Guidelines for ADL}

High exercise capacity is a strong predictor of active daily living, high QOL, and favorable prognosis both in patients with cardiac disease and healthy individuals. ${ }^{337}$ Appropriate reference values should be used to assess exercise capacity. Table 57 shows the data recently published as reference values for exercise capacity expressed as the AT and peak $\dot{\mathrm{VO}}_{2}$ of people in Japan. ${ }^{338}$ Exercise capacity decreases linearly as a function of age, and is lower in women than in men. Oxygen uptake is smaller during cycle ergometer exercise than it is during treadmill ergometer exercise. In Japan, exercise intensity is most commonly estimated by METs. Recently, energy requirements of ADL and exercises for people in Japan were published (Table 58). ${ }^{339}$ In patients participating cardiac rehabilitation programs, exercise capacity should be assessed accurately using these reference data to prescribe appropriate exercise prescriptions.

\section{(1) Instructions on ADL}

Patients are recommended to perform aerobic exercise below the AT such as walking and cycling that use large muscles and are correspond to about 3 4 METs in healthy individuals. The MHLW proposed a unit for the amount of exercise (expressed 


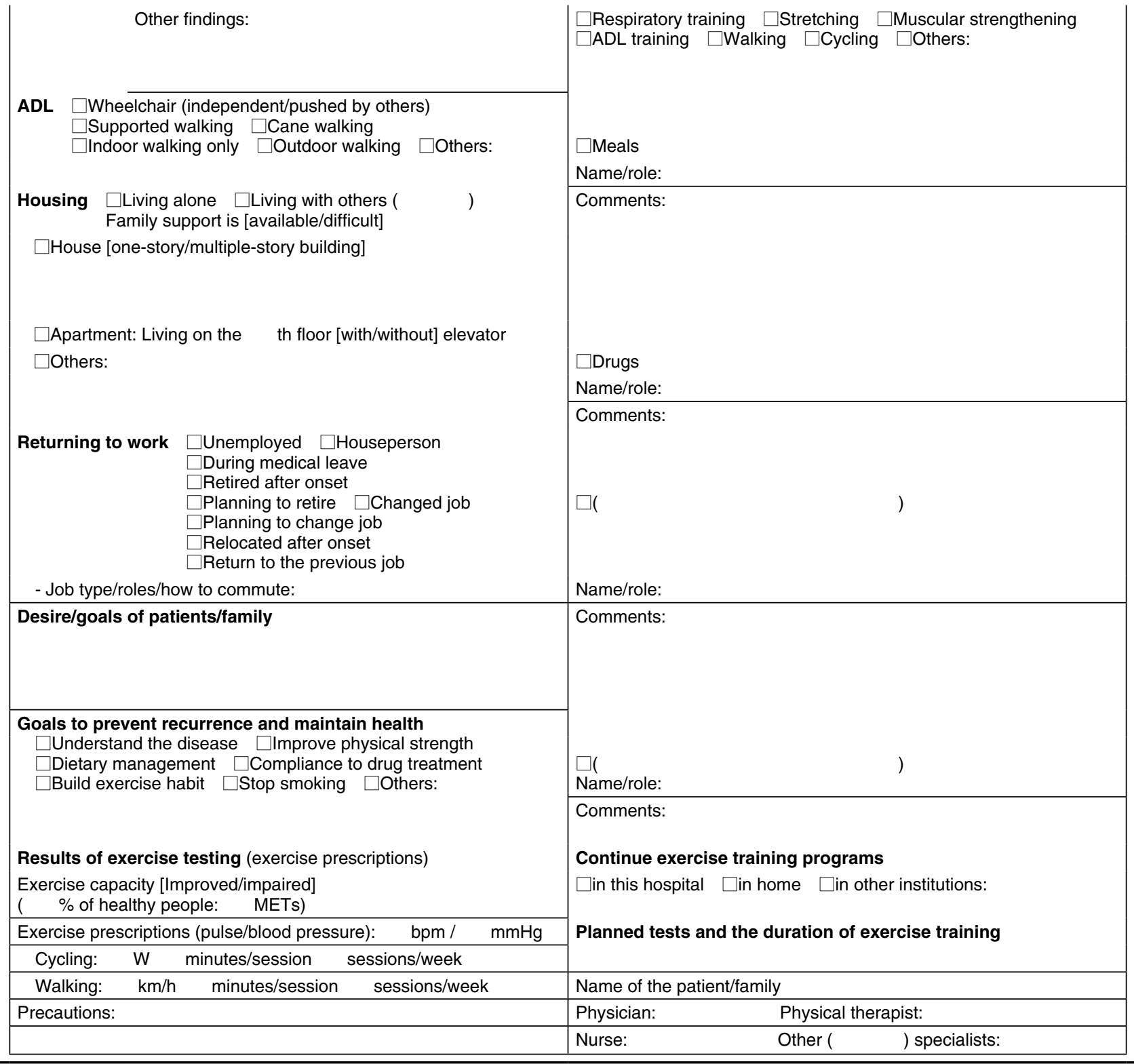

Source: http://www.mhlw.go.jp/seisakunitsuite/bunya/kenkou_iryou/iryouhoken/iryouhoken15/dl/2-22-5.pdf. ${ }^{325}$

as Ex) in the Exercise Guide 2006 ("Exercise and Physical Activity Guide for Health Promotion 2006") as a measure of energy consumption during exercise to take due account of the importance of energy consumption as well as exercise intensity (expressed as METs). ${ }^{103,340}$ The amount of exercise is expressed as the product of intensity and time of exercise, and may be used to calculate energy consumption during exercise.

[Formula for calculating the amount of exercise]

Amount of exercise $(\mathrm{Ex})=$ Intensity of physical activities (METs) $\times$ Time (hours)

[Examples]

- Amount of exercise when physical activity corresponding to 3 METs is performed for 1 hour: 3 METs $\times 1$ hour $=3 \mathrm{Ex}$ (METs-hour)

- Amount of exercise when physical activity corresponding to 6 METs is performed for 30 minutes: 6 METs $\times 1 / 2$ hour $=3$ Ex (METs-hour)
[Formula to calculate the amount of energy consumed during exercise]

Amount of energy consumed during exercise $(\mathrm{kcal})=1.05 \times$ Ex $\times$ body weight $(\mathrm{kg})$

The ACSM and the AHA recommend that healthy individuals perform moderate-intensity aerobic exercise for at least 30 minutes/session 5 days a week or high-intensity dynamic exercise for at least 20 minutes/session 3 days a week in addition to low-intensity exercise such as walking and housework in daily life. In addition to energy consumption during exercise, non-exercise activity thermogenesis (NEAT), the energy expenditure of all physical activities, such as housework, other than exercise, is gaining interest in preventing obesity. ${ }^{341}$

Patients with impaired exercise capacity due to cardiac dysfunction such as MI should perform exercise at intensities lower than healthy individuals. Middle-aged and elderly patients with a high risk for cardiovascular and atherosclerotic 


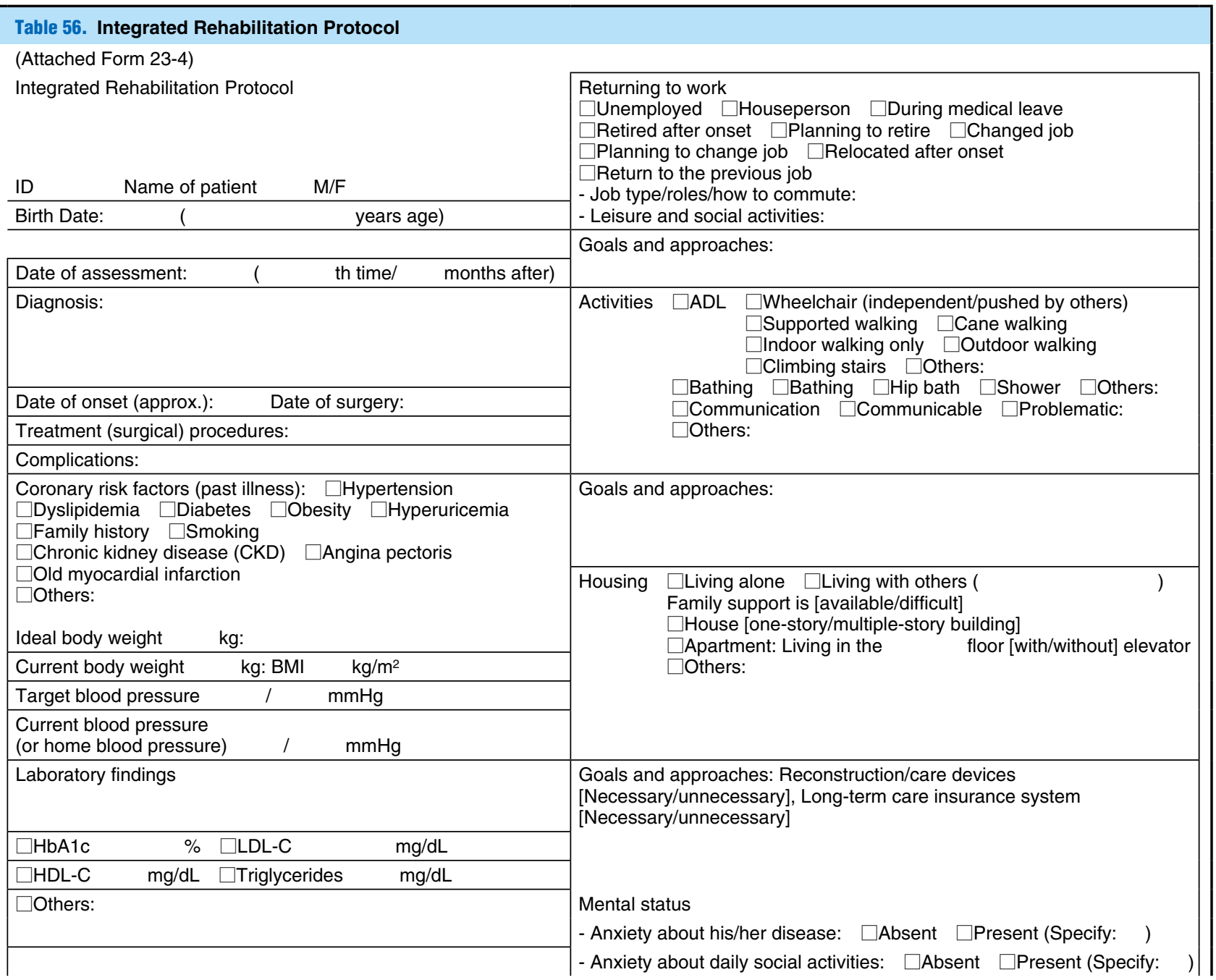

(Table 56 continued the next page.)

diseases should undergo exercise stress tests to assess exercise capacity and confirm the absence of cardiovascular dysfunction during exercise to ensure safe and effective exercise training programs.

\section{Mental Stress, Sleep Disorders, and Returning to Work (1) Life-Work Balance and Stress Control}

Cardiovascular diseases are most commonly observed among middle-aged and elderly patients. Their social roles and busy lifestyle do not always induce strong mental stress, but rather often motivate them to live better and enjoy life. The loss experiences in old age may significantly affect mental function and cause depressive state and other psychological symptoms. ${ }^{342}$ When stress control is considered for patients in this age group, physicians should encourage patients to build better work-rest balance rather than to quit or reduce work hours.

In a study of the relationship of work and sleeping hours with the incidence of AMI in Japan, the risk of AMI was 1.9 times higher in patients who worked $\geq 61$ hours/week than those who worked $\leq 40$ hours/week during 1 month. In another study of the relationship of short-term sleep with the incidence of cardiovascular diseases in Japan, a sleeping time of about $<6$ hours/day was associated with a higher risk of cardiovascular diseases. ${ }^{343}$
Healthcare professionals should be aware that hardworking, high responsibility, perfectionistic, stubborn, and idealistic individuals tend to overwork and lose work-rest balance and therefore, they should encourage such individuals to rest appropriately and learn how to manage stress with techniques such as relaxation methods.

Women tend to suffer physical and mental disorders more commonly than men as women experience significant changes in hormone levels at different life stages such as menses, pregnancy, childbirth, and menopause; stress response also varies accordingly. ${ }^{344,345}$ In general, the prevalence of depression is higher in women than in men, although the incidence of suicide is higher in men (especially single men) than in women.

\section{(2) Sleep Disorder}

In addition to sleep shortage, many types of sleep disorders including insomnia increase the risk of cardiovascular diseases. ${ }^{346}$

Symptoms of insomnia include difficulty falling asleep, difficulty maintaining sleep, early morning awakening, and poor perceived quality of sleep, and may cause physical symptoms such as palpitation, shortness of breath, decreased weight, headache, dizziness, gastrointestinal disorder, back pain, stiff neck, and chronic fatigue, as well as psychological symptoms 


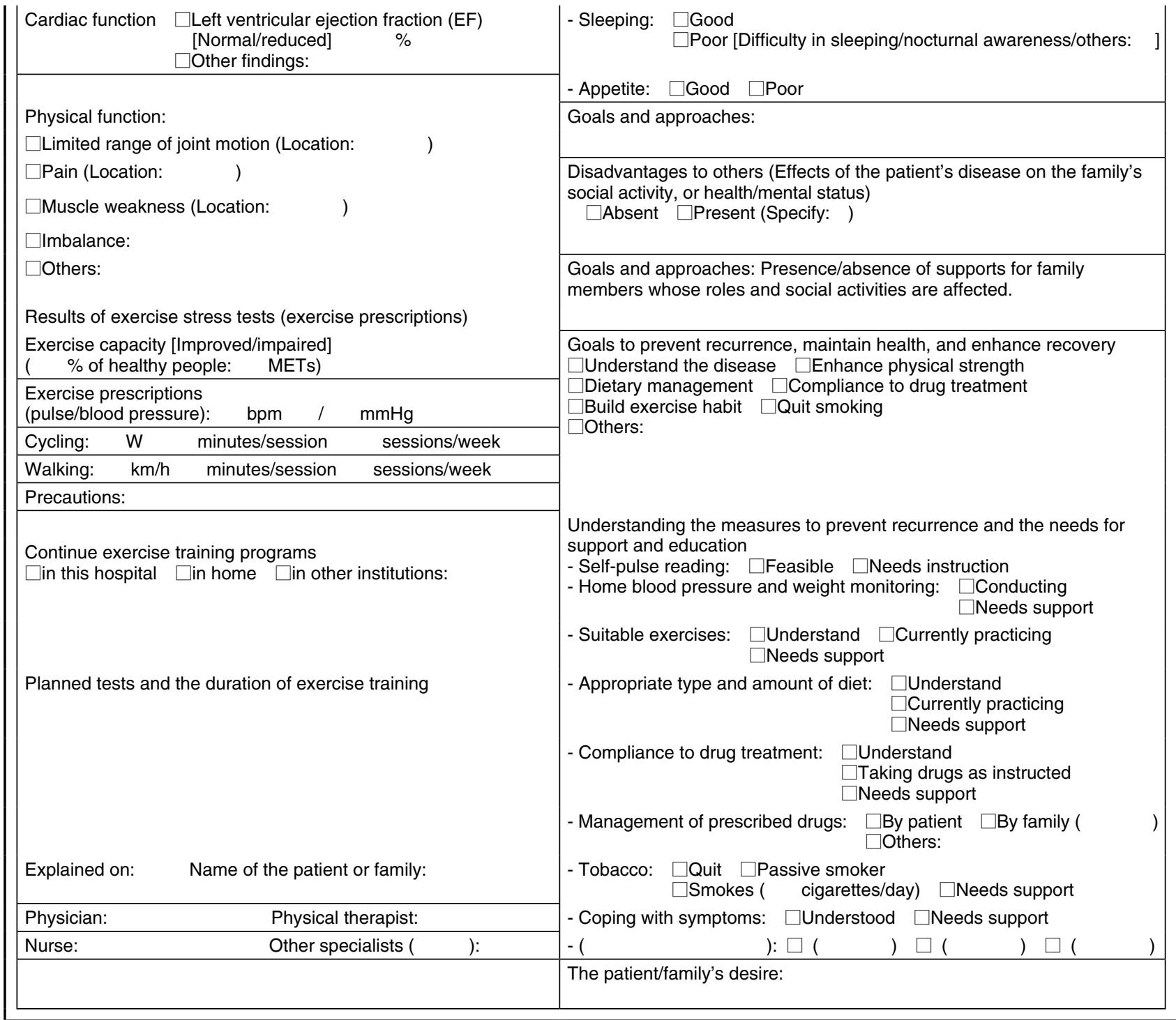

Source: http://www.mhlw.go.jp/seisakunitsuite/bunya/kenkou_iryou/iryouhoken/iryouhoken15/dl/2-22-7.pdf. ${ }^{326}$

such as loss of motivation, irritability, impaired concentration, and decreased attentiveness. ${ }^{347}$ Symptoms of insomnia also may impair recovery from fatigue and cardiovascular diseases, and may increase the risk of recurrent cardiovascular diseases.

Symptoms of sleep disorders are not limited to insomnia. Physicians treating patients with cardiovascular diseases should refer to guidelines for primary care physicians (Figure 13) or other appropriate materials or should collaborate with specialists. ${ }^{348}$

Symptoms of insomnia are effectively treated with drug treatment ${ }^{349,350}$ as well as mental therapy, such as cognitive behavioral therapy. ${ }^{351}$ And patient education such as improving the patient's environment to ensure good sleep.

Sleep apnea syndrome and other SDB (See Section IV-5-32 "Patients with Sleep-Disordered Breathing Associated with Heart Failure") are important factors that exacerbate cardiac diseases. Physicians should follow up patients who return to work and assess their job situation and sleeping conditions. ${ }^{346,352}$
(3) Management of Patients Who Have Returned to Work Patients returning to work may have mental burdens as well as physical burdens. During absence from work, patients can easily get worried about whether they will be able to work as before, or whether can adjust to a new working environment (e.g., transfer to other sections), and tend to concern about not working, blame themselves for their disease. Physicians should give considerable attention to the patients who returned to work, since they tend to overwork in order not to give their colleagues further trouble.

\section{Machines, Equipment and Facilities for Exercise Training}

\section{Machines for Exercise Training (Table 59)}

Class I

1. It is recommended that facilities for exercise training be equipped with both aerobic exercise machines, such as tread- 


\begin{tabular}{|c|c|c|c|c|c|c|c|c|c|}
\hline \multicolumn{10}{|c|}{ Cycle ergometer } \\
\hline \multirow[t]{2}{*}{ M } & AT & 19.5 & 18.4 & 17.4 & 16.4 & 15.4 & 14.4 & 3.41 & 285 \\
\hline & Peak $\dot{\mathrm{VO}}_{2}$ & 36.8 & 34.1 & 31.4 & 28.7 & 25.9 & 23.2 & 6.35 & 272 \\
\hline \multirow[t]{2}{*}{$\mathrm{F}$} & AT & 18.0 & 17.3 & 16.6 & 15.9 & 15.2 & 14.5 & 3.09 & 260 \\
\hline & Peak $\dot{V O}_{2}$ & 31.5 & 29.5 & 27.5 & 25.6 & 23.6 & 21.7 & 5.42 & 251 \\
\hline \multicolumn{10}{|c|}{ Treadmill } \\
\hline \multirow[t]{2}{*}{ M } & AT & 26.4 & 24.7 & 22.9 & 21.2 & 19.5 & 17.8 & 4.49 & 102 \\
\hline & Peak $\mathrm{VO}_{2}$ & 50.9 & 45.8 & 40.7 & 35.6 & 30.5 & 25.4 & 9.78 & 97 \\
\hline \multirow[t]{2}{*}{$\mathrm{F}$} & AT & 20.8 & 20.1 & 19.4 & 18.7 & 18.0 & 17.3 & 3.11 & 102 \\
\hline & Peak $\dot{V}_{2}$ & 36.5 & 34.4 & 32.3 & 30.2 & 28.2 & 26.1 & 5.20 & 93 \\
\hline
\end{tabular}

AT, anaerobic threshold; F; female; $\mathrm{M}$, male; peak $\mathrm{VO}_{2}$, peak oxygen uptake; SD, standard deviation.

Exercise capacity is expressed with the oxygen uptake at the AT and peak $\mathrm{VO}_{2}(\mathrm{~mL} / \mathrm{min} / \mathrm{kg})$ estimated by age and sex, according to the regression analysis model described in: Itoh $\mathrm{H}$, et al. Heart rate and blood pressure response to ramp exercise and exercise capacity in relation to age, gender, and mode of exercise in a healthy population. $J$ Cardiol 2013; 61: 71-78. ${ }^{338}$

\begin{tabular}{|c|c|c|c|c|}
\hline & \multicolumn{2}{|c|}{ Activities of daily living } & \multicolumn{2}{|l|}{ Exercises } \\
\hline & & METs & & METs \\
\hline Low intensity (1.0 3.0 METs) & $\begin{array}{l}\text { Office work } \\
\text { Sweeping } \\
\text { Dishwashing } \\
\text { Watering (using one hand) } \\
\text { Watering (using both hands) }\end{array}$ & $\begin{array}{l}1.7 \pm 0.3 \\
2.6 \pm 0.3 \\
2.7 \pm 0.4 \\
2.9 \pm 0.4 \\
3.0 \pm 0.4\end{array}$ & $\begin{array}{l}\text { Stretching (standing) } \\
\text { Stretching (sitting) } \\
\text { Resistance exercise (standing) }\end{array}$ & $\begin{array}{l}1.6 \pm 0.3 \\
1.8 \pm 0.2 \\
2.6 \pm 0.5\end{array}$ \\
\hline Moderate intensity (3.0 4.5 METs) & $\begin{array}{l}\text { Vacuuming } \\
\text { Hanging washed clothes } \\
\text { Mopping the floor } \\
\text { Pruning } \\
\text { Cleaning the toilet } \\
\text { Gardening } \\
\text { Spreading bedding } \\
\text { Putting bedding in a closet } \\
\text { Window cleaning } \\
\text { Weeding }\end{array}$ & $\begin{array}{l}3.2 \pm 0.4 \\
3.2 \pm 0.4 \\
3.3 \pm 0.5 \\
3.5 \pm 0.7 \\
3.6 \pm 0.7 \\
3.7 \pm 0.7 \\
3.8 \pm 0.6 \\
3.9 \pm 0.6 \\
4.0 \pm 0.6 \\
4.1 \pm 0.3\end{array}$ & $\begin{array}{l}3 \mathrm{~km} / \mathrm{h} \text { walking } \\
\text { Resistance exercise (sitting) } \\
\text { Aerobic exercise (standing) } \\
4 \mathrm{~km} / \mathrm{h} \text { walking } \\
\text { Aerobic exercise (sitting) }\end{array}$ & $\begin{array}{l}3.1 \pm 0.5 \\
3.2 \pm 0.4 \\
3.4 \pm 0.8 \\
3.7 \pm 0.3 \\
3.7 \pm 0.5\end{array}$ \\
\hline High intensity ( $\geq 4.5$ METs) & $\begin{array}{l}\text { Cleaning the bathroom } \\
\text { Lawn mowing } \\
\text { A } 4 \mathrm{~km} / \mathrm{h} \text { walking with a } 5 \mathrm{~kg} \text { load } \\
\text { Plowing a field } \\
\text { A } 4 \mathrm{~km} / \mathrm{h} \text { walking with a } 10 \mathrm{~kg} \text { load } \\
\text { Carry a heavy load* } \\
\text { Lifting stairs }\end{array}$ & $\begin{array}{l}4.9 \pm 0.6 \\
5.0 \pm 0.3 \\
5.1 \pm 1.0 \\
5.3 \pm 1.0 \\
5.5 \pm 1.0 \\
6.5 \pm 1.1 \\
6.9 \pm 1.0\end{array}$ & $\begin{array}{l}\text { Radio taiso** } \\
\text { Ping-pong } \\
6 \mathrm{~km} / \mathrm{h} \text { walking } \\
\text { Badminton }\end{array}$ & $\begin{array}{l}4.6 \pm 0.4 \\
5.1 \pm 1.0 \\
5.3 \pm 0.6 \\
7.8 \pm 1.9\end{array}$ \\
\hline
\end{tabular}

*(i.e., a case of 24 cans of $350 \mathrm{~mL}$ each)

$* *$ (a set of basic calisthenics common in Japan)

METs, metabolic equivalent units.

Adapted from Itoh $\mathrm{H}$, et al. Heart rate and blood pressure response to ramp exercise and exercise capacity in relation to age, gender, and mode of exercise in a healthy population. $J$ Cardiol $2013 ; 61: 71-78,{ }^{339}$ with modification.

mill or cycle ergometer, and resistance training devices, such as resistance training machine, dumbbells or weights. (Level of Evidence: $\mathrm{C}$ )

2. It is recommended that facilities for exercise training be equipped with ECG monitors, blood pressure monitors, automated external defibrillators (AEDs), and emergency carts. (Level of Evidence: C)

\section{Class Ila}

1. A standard 12-lead ECG is not usually used during exercise training. However it is appropriate that a 12-lead ECG monitor is available in the first-aid room of each exercise training facility to use for patients who experienced chest pain. (Level of Evidence: C)
2. It is appropriate that facilities for exercise training be equipped with simplified blood glucose monitors, percutaneous oxygen saturation monitors, and spirometry devices. (Level of Evidence: C)

3. It is appropriate that facilities for exercise training be equipped with video players, DVD players, personal computers, and projectors for patient education and also equipped with simplified pulse monitoring devises and Borg scale panel to monitor exercise intensity or ratings of perceived exertion. Physical activity monitoring devises and salt intake monitoring devices are also useful for management of patient's lifestyle. (Level of Evidence: B) 


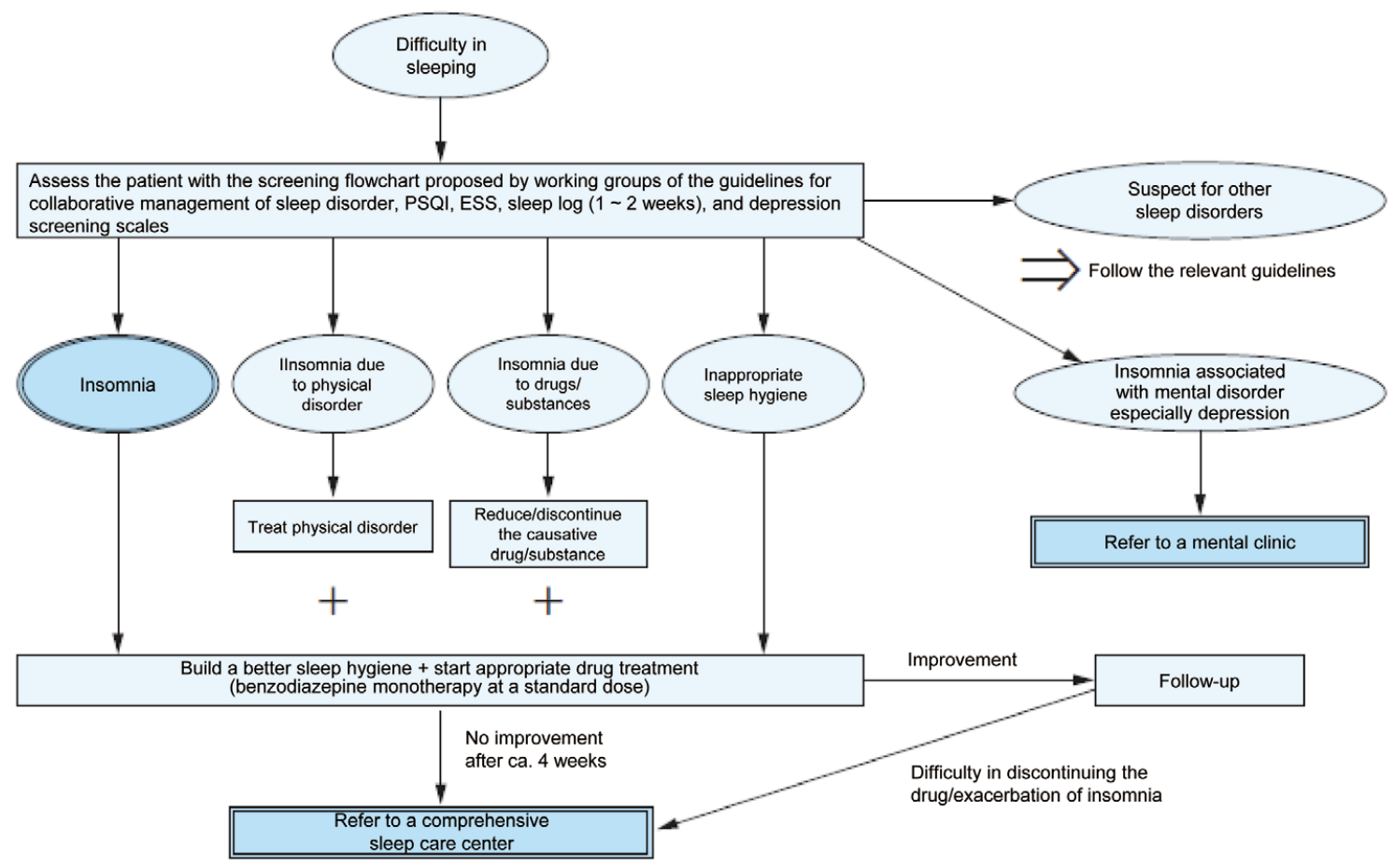

Figure 13. The guidelines for the diagnosis, treatment and collaborative management of sleep disorder in general clinics. ESS, Epworth Sleepiness Scale; PSQI, Pittsburgh Sleep Quality Index. Adapted from Yamadera W, et al. Guidelines for the diagnosis, treatment and collaborative management of sleep disorder. Suimin Iryo 2008; 2: 285-289.348

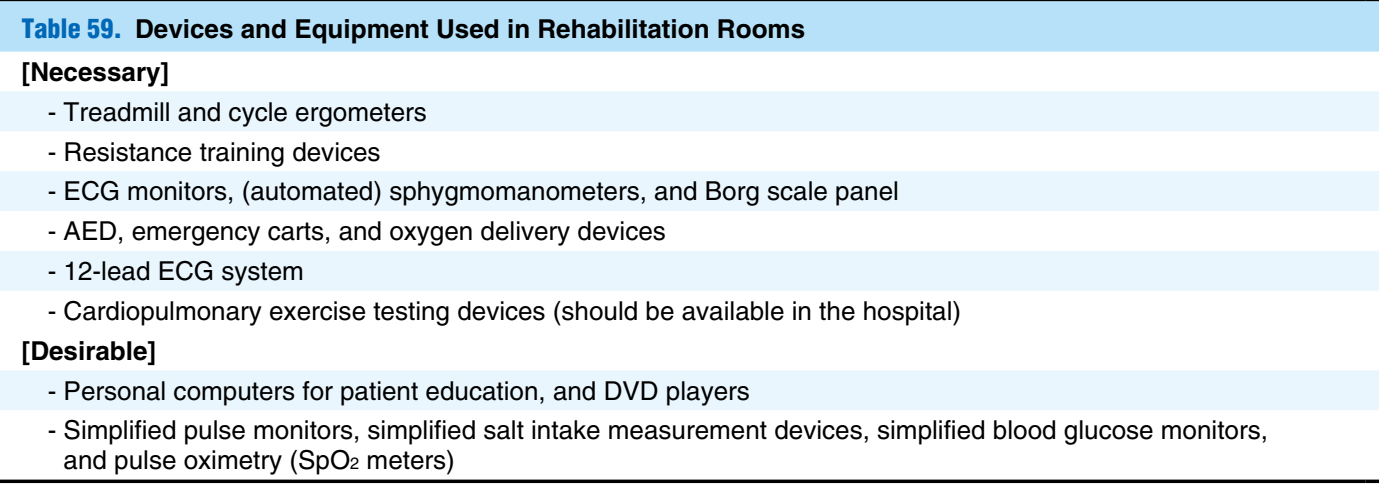

$\mathrm{AED}$, automated external defibrillator; $\mathrm{SpO}_{2}$, percutaneous arterial oxygen saturation.

\section{(1) Equipment for Assessment of Physical Function}

Graded exercise test, especially CPX with expiratory gas analysis, is useful in assessing exercise capacity. Skeletal muscle strength may be assessed based on one repetition maximum or isokinetic muscle strength.

\section{(2) Equipment for Exercise Training}

Treadmill or cycle ergometers are used in aerobic exercise training. Approximately 10 types of muscle training, including upper and lower extremity muscles, should be performed for resistance exercise training. The facility should be equipped with appropriate resistance training machines, weights, or other equipment. Pulse monitoring devices and accelerometers are also useful in home-based exercise to monitor exercise intensity or physical activity.

\section{(3) Vital Sign Monitors}

During exercise, ECG, blood pressure, ratings of perceived exertion, and other parameters should be monitored. Although a 12-lead ECG is not necessary during exercise, it should be available in the first-aid room of training facilities to measure ECG in case of emergency.

\section{(4) Equipment to Respond to Cardiac Events During Exercise}

As cardiac events may occur during exercise training, facili- 
ties for exercise training must be equipped with AEDs and emergency carts. Because patients are often complicated with diabetes or respiratory disorders, simplified blood glucose monitors and percutaneous oxygen saturation monitors must be available as well.

\section{(5) Equipment for Lifestyle Modification}

Cardiac rehabilitation programs compose salt restriction, smoking cessation, and nutritional modification. Salt intake measurement devices and home sphygmomanometers are useful for monitoring salt intake in salt restriction program, and information materials of healthy food such as nutritional supplements are useful in nutritional modification program.

\section{Future Prospects of Exercise Training}

\section{Collaboration With Local Facilities for Exercise Training: Current Situations and Future Prospects}

\section{Collaboration With Local Facilities for Exercise Training: Current Situations and Future Prospects} Cardiac rehabilitation, especially exercise training, should be continued after the patient leaves the hospital and returns to work. In Japan, medical institutions have tried to collaborate with non-profit organizations and private exercise facilities to develop systems for maintenance-phase cardiac rehabilitation, but no substantial results have been achieved.

\section{(1) Governmental Activities Regarding Primary and Secondary Prevention of Cardiovascular Diseases}

The government of Japan has not taken any particular measures for ensuring maintenance-phase cardiac rehabilitation, but has provided measures mainly for encouraging primary prevention of cardiovascular diseases such as the "first National Health Promotion Measures" in 1978, the "Active 80 Health Plan (the second National Health Promotion Measures)" in 1988, and the "Health Japan 21 (National Health Promotion in the 21st Century)" starting in 2000. These measures, proposed by the MHLW are taken to prevent the development of cardiovascular diseases among healthy individuals. However, the government has not proposed any health promotion measures for patients with cardiovascular diseases.

\section{(2) Support of and Collaboration With Private Facilities for Exercise Training}

It has been expected that maintenance-phase exercise programs be provided in existing health promotion facilities (e.g., fitness clubs, public gymnasiums, and healthcare facilities) and health promotion activities to help patients continue exercise training. However, there are a number of problems with this approach. For example, medical institutions and exercise facilities must develop appropriate methods to share patient information, and exercise facilities should be staffed with trained individuals capable of understanding patient medical information to provide appropriate exercise training programs. To solve these programs, medical institutions and health promotion facilities should build strong networks to educate health fitness programmers on cardiac rehabilitation and to provide and share information. ${ }^{353,354}$ In addition, a learning and certification system should be established to train qualified individuals to understand and provide appropriate exercise training as a secondary preventive measure for patients with cardiovascular diseases. ${ }^{355,356}$

\section{(3) Management of Facilities for Exercise Training in Medical Institutions}

In Japan, medical institutions may have health promotion facilities that provide long-term training programs that is eligi- ble for the healthcare costs deduction if the institutions meet the requirements of Article 42 of the Medical Service Act or are certified as designated facilities for exercise training. ${ }^{357-359}$

\section{Disease Management Through Hospital-Clinic Cooperation \\ (1) Community Collaborative Clinical Pathways and Cardiac Rehabilitation}

Community collaborative clinical pathways are gaining attention as new, community-based disease management programs for patients with angina pectoris and MI. However, the problems are that cardiac rehabilitation programs after discharge from hospital are not emphasized and such pathways are reported to be not prevalent. ${ }^{360,361}$

\section{Ways to Continue Exercise Training}

\section{(1) Ways to Maintain Motivation}

Although it is often difficult for individuals to continue exercise training for a long period of time, behavioral interventions to increase self-efficacy and develop self-management skills have been reported to be the most effective way to motivate patients with cardiac diseases. ${ }^{362-364}$

\section{(2) Community-Based Rehabilitation Programs}

1) Community-Based Rehabilitation Programs in Other Countries

Community-based rehabilitation programs are popular in Germany and Scandinavian countries. In Germany, patients participate in community-based, training groups called ambulante Herzgruppe (AHG), and continue cardiac rehabilitation programs for life. The feature of AHG is that programs provided by AHG are covered by health insurance system in Germany. In Finland, public hospitals provide community-based cardiac rehabilitation programs targeting different cardiovascular diseases. ${ }^{365}$

In the United States, because cardiac rehabilitation programs are usually not covered by health insurances, various attempts have been made to develop cost-effective, community-based cardiac rehabilitation programs. One of such attempt is the INTERxVENT, a Web-based program. ${ }^{365}$

\section{2) Community-Based Rehabilitation Programs in Japan} (Figure 14)

Good relationships should be built between medical institutions and local facilities for exercise training to establish community-based cardiac rehabilitation programs in Japan.

Typical examples of community-based cardiac rehabilitation programs models are the Japan Heart Club (JHC), a nonprofit organization (Figure 15), and the Japan Medical Fitness (JMF) network at Kansai Medical University. ${ }^{355,366,367}$ 


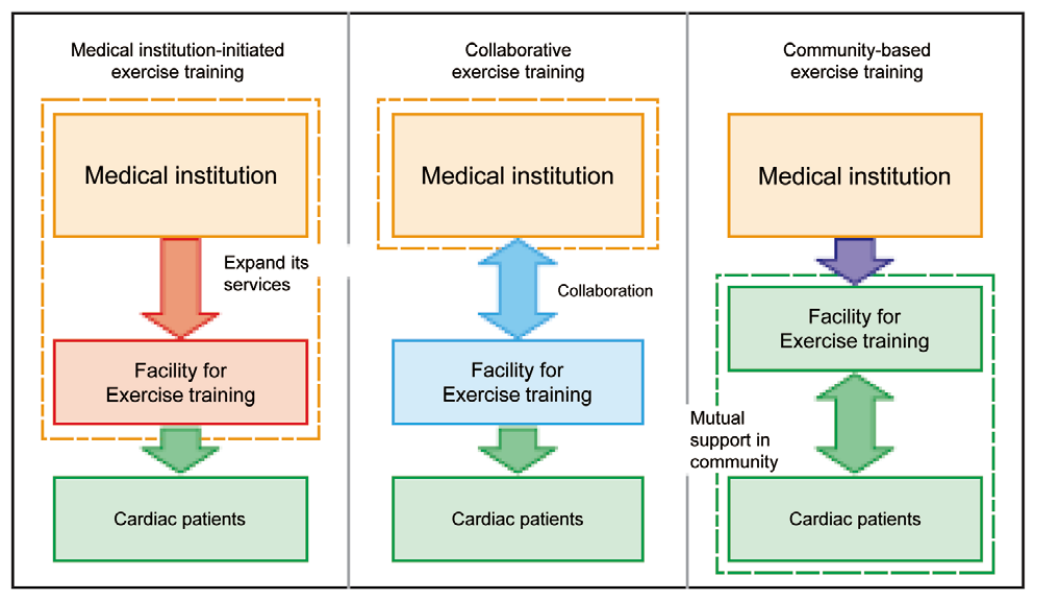

Figure 14. Patterns of collaboration between medical institutions and facilities for exercise training.

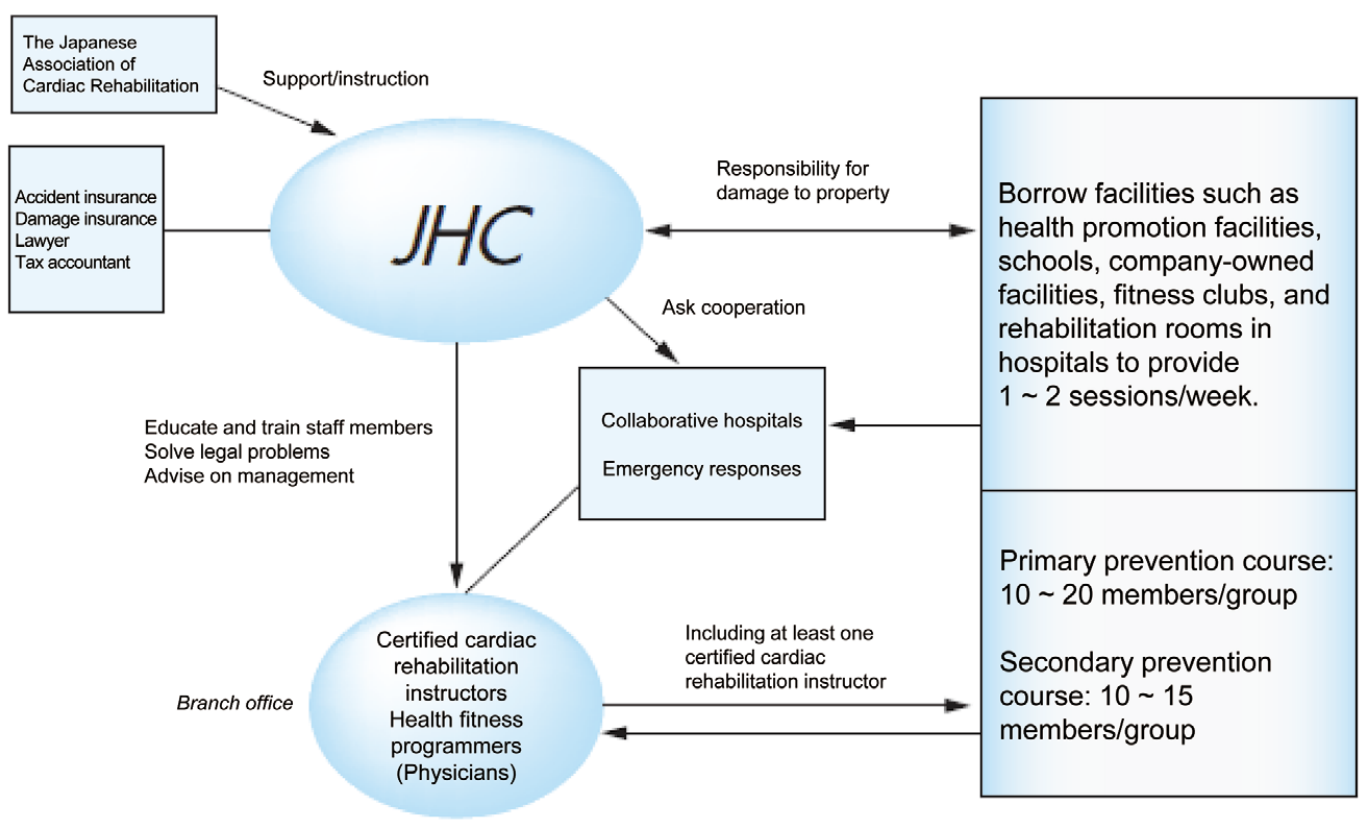

Figure 15. Medics club of the Japan Heart Club (JHC), a non-profit organization.

\section{(3) Home-Based Cardiac Rehabilitation Programs Using Information and Communication Technology (Figure 16)}

Due to the advancement of programs using information and communication technology (ICT) such as the internet, ICTbased healthcare services (i.e., E-Health) have been introduced to provide information about health and diseases, conduct remote medical care, share health and medical information, and manage diseases, among others.

Recently, ICT-based lifestyle interventions have been attempted. In this system, data on body weight and step count are automatically sent to the central database when patients weigh themselves on a scale or wear pedometers. ${ }^{368}$ Physi- cians and other attending individuals tasked to instruct patients may access to the database to monitor the body weight and step count of their patients. It has been demonstrated that ICTbased lifestyle interventions are beneficial helping the patient to reduce body weight, stop using tobacco, and continue exercise training programs. ${ }^{369-372}$

In Australia, the Australian e-Health Research Centre (AEHRC) is conducting a RCT of patients recovering from a MI, comparing an ICT-based cardiac rehabilitation program, in which all elements of cardiac rehabilitation (education, instruction, setting goals, and counseling) are enacted using mobile phones and the Internet, with a conventional homebased cardiac rehabilitation program. ${ }^{373}$ 


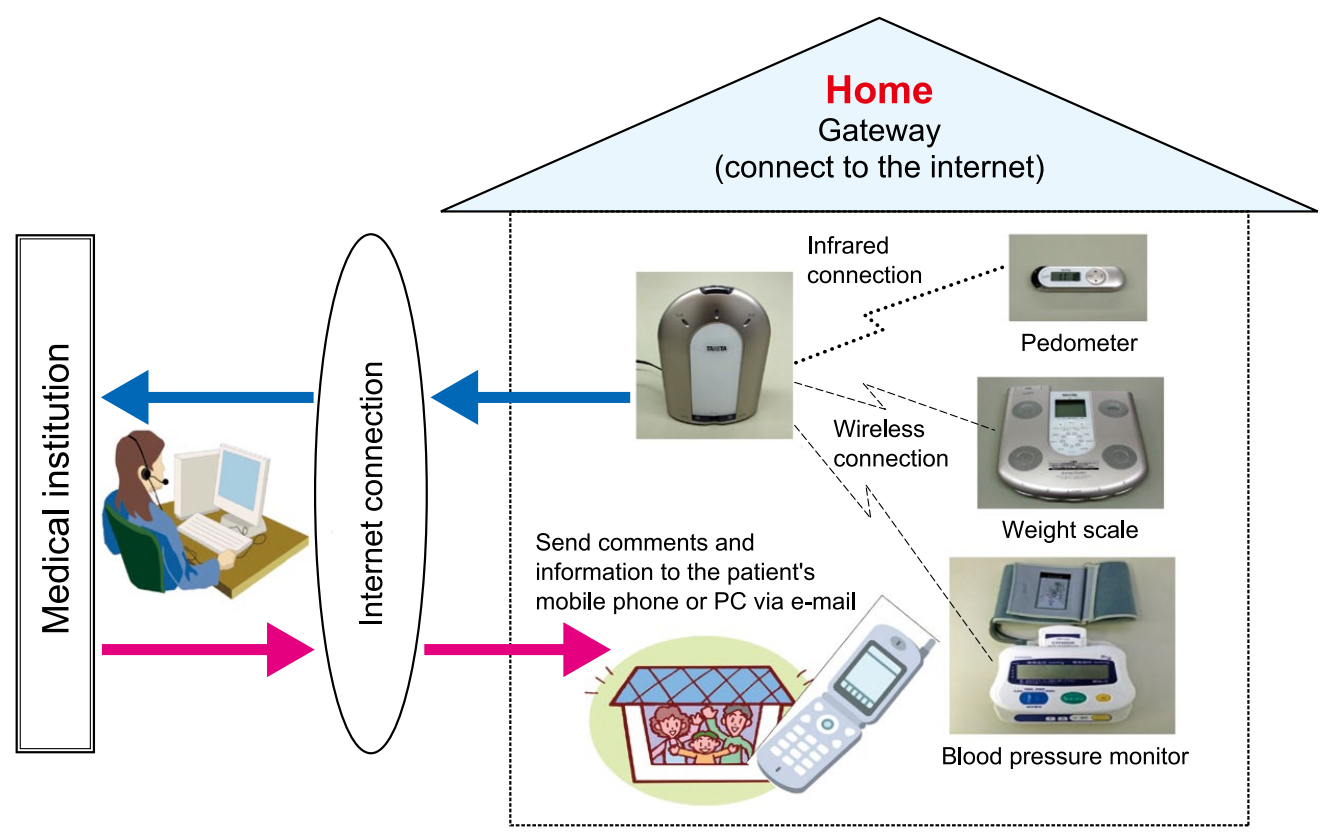

Figure 16. Example of remote exercise instruction/management system using information and communication technology (ICT).

\section{Current Medical Service Fees for Cardiac Rehabilitation and Future Goals}

\section{Change Over Time in the Coverage of Cardiac Rehabilitation Programs by the Health Insurance System in Japan (Table 60) ${ }^{374}$}

In the medical service fee revision in April 2010, diseases indicated for rehabilitation programs and fees for each disease category were listed (Table 61), ${ }^{375}$ and the criteria for medical institutions providing rehabilitation programs were revised (Table 62). ${ }^{375}$

The following points were revised in April 2010, and Table 63 lists remarks on medical service fees for "cardiovascular rehabilitation".

1. In medical institutions providing "cardiovascular rehabilitation (I)", cardiologists or cardiovascular surgeons were required to be available for 24 hours a day and 365 days a year before the revision, but are now required to be available during the period when rehabilitation sessions are conducted.

2. Physical therapists and nurses dedicated to "cardiovascular rehabilitation" may be involved in rehabilitation programs for other diseases during the period when they are not involved in "cardiovascular rehabilitation".

3. Functional training rooms for cardiovascular rehabilitation may be used as rooms for rehabilitation for patients with other diseases, provided that the rooms meet the criteria for relevant rehabilitation rooms. When a healthcare professional dedicated to "cardiovascular rehabilitation" provides intergroup communication therapy and rehabilitation ses- sions to patients with cardiovascular diseases and to other patients, he or she may provide a total of around 18 service units/day and $\leq 108$ service units/week per physician, where 1 service unit is defined as a 20 -minute service in terms of "cardiovascular rehabilitation".

4. When a patient with cardiovascular diseases starts rehabilitation during hospitalization, services during the first 30 days of rehabilitation are subjected to "early-phase rehabilitation premium" with a NHI reimbursement of 45 points per service unit, which was 30 points before the revision.

5. A NHI premium of 100 points was introduced for "continuous respiratory gas analysis" during CPX to prescribe appropriate exercise prescriptions.

The indications for "cardiovascular rehabilitation" were expanded to include patients with AMI, those with angina pectoris, those who have undergone open heart surgery, those with a large vessel disease (aortic dissection, dissecting aortic aneurysm, or after large vessel surgery), those with chronic heart failure, and those with peripheral arterial occlusive disease, among others. In the medical service fee revision in April 2012,365 the following changes were made for the criteria of medical institutions providing cardiovascular rehabilitation (Table 62). ${ }^{375}$

1. The "early-phase rehabilitation premium" was reduced from 45 points to 30 points, and the "initial phase premium" of 45 points was newly set for rehabilitation services during the first 14 days. With this revision, the NHI reimbursement of "cardiovascular rehabilitation" during the first 14 days is increased to a total of 75 points per unit consisting of 30 points of the "early-phase rehabilitation premium" 
Table 60. Changes Over Time in NHI Reimbursement for Cardiovascular Rehabilitation in Japan

1988 Cardiac rehabilitation was included in the NHI reimbursement list (as "fees for physical therapy for cardiac disease", covering only patients after acute myocardial infarction during the first 3 months, 335 points).

1992 The title was changed to "fees for cardiac rehabilitation", and the point value was increased (335 $\rightarrow 480$ points).

1996 The approval point value was increased ( $480 \rightarrow 530$ points), duration was extended ( $3 \rightarrow 6$ months), and indications were expanded (patients with acute myocardial infarction, those with angina pectoris, and those who had undergone open heart surgery).

1998 The point value was increased $(530 \rightarrow 550$ points).

2004 The criteria for hospitals providing cardiac rehabilitation were relaxed to exclude the criterion "hospitals should satisfy the requirement for hospitals that can claim fees for designated ICU treatment or fees for critical care treatment".

2006 Fees for disease-specific rehabilitations were introduced, and the fees for cardiovascular rehabilitation in the approved facilities category I and II were set at 250 points/20 minutes and 100 points/20 minutes, respectively. Namely, the point value for a standard 60 -minute session was increased for category I ( $550 \rightarrow 750$ points) and decreased for category II ( $550 \rightarrow 300$ points). The duration covered was decreased from 6 months to 150 days.

2007 The criteria for patients for whom the fees for rehabilitation are covered by NHI without limitation were defined. The fee for rehabilitation and medical management was newly introduced. Disease-specific rehabilitation fees were revised. A step-down pricing policy was introduced.

2008 Disease-specific rehabilitation fees were revised. The fees for category I were changed from 250 to 200 points/20 minutes, while the fees for category II were unchanged at 100 points/20 minutes. The fee for a 60 -minute session was changed from 750 to 600 points for category I and was unchanged at 300 points for category II. The fee for rehabilitation and medical management, the step-down pricing policy, and the maximum duration of $\mathrm{NHI}$ coverage were abolished. The indications were extended to include large vessel diseases (patients with aortic dissection, those with dissecting aortic aneurysm, and those who had undergone large vessel surgery), chronic heart failure, and peripheral arterial occlusive disease in addition to the existing indications of those with acute myocardial infarction, those with angina pectoris, and those who had undergone open heart surgery. An early-phase rehabilitation premium was introduced for the first 30 days of rehabilitation ( 30 points).

2010 In hospitals providing rehabilitation after heart or large vessel diseases, physicians providing cardiovascular or cardiovascular surgery services are no longer required to be available for 24 hours a day and 365 days a year. The requirement for physical therapists responsible for rehabilitation in patients with cardiovascular diseases, but who had not been allowed to be responsible for or dedicate to other categories of rehabilitation, were changed to allowed them to provide rehabilitation sessions for patients with other diseases. The term to define the area used for functional training was changed from "room" to "space" to ease the requirement for the location of functional training. The early-phase rehabilitation premium was increased from 30 to 45 points. A premium of 100 points was introduced for continuous respiratory gas analysis during cardiopulmonary exercise testing.

2012 The early-phase rehabilitation premium was decreased from 45 to 30 points. The initial phase premium (45 points) was introduced for the first 14 days. During the first 14 days, the point value was increased to 75 points ( 30 points for the early-phase rehabilitation premium and 45 points for the initial phase premium), which was higher in the previous version ( 45 points). The $\mathrm{NHI}$ reimbursement list added samples of protocols for inpatients and outpatients and summarized protocols for cardiovascular rehabilitation.*

${ }^{*}$ See Tables 54 56. 1 point=10 yen. NHI, National Health Insurance.

Adapted from Kohzuki M. Cardiac rehabilitation and health insurance treatment. Junkanki Naika 2011; 69: 267-274,,374 with modification.

Table 61. NHI Reimbursement for Disease-Specific Rehabilitation

Typical indications

Rehabilitation fees (I) Points per 1 unit (20 minutes)

Rehabilitation fees (II) Points per 1 unit (20 minutes)

Rehabilitation fees (III)

Points per 1 unit

(20 minutes)

Standard maximum

length of NHI coverage

\section{Cardiovascular rehabilitation \\ Musculoskeletal rehabilitation}

Acute myocardial infarction, angina pectoris, recovery from open heart surgery, large vessel diseases, chronic heart failure, and peripheral arterial occlusive diseases, among others 200

100

Extremity trauma, recovery after surgery for fractures, and joint contracture due to burn scar, among others

\section{Pulmonary rehabilitation}

Pneumonia or atelectasis, and Grade II or severer chronic obstructive pulmonary disease, among others

\section{Rehabilitation after} cerebrovascular diseases or other diseases (disuse syndrome)

Cerebrovascular diseases, and brain injury, among others

Source: Notifications from the Medical Economics Division, Health Insurance Bureau, MHLW regarding the revision of NHI reimbursement list in 2012. URL: http://www.mhlw.go.jp/seisakunitsuite/bunya/kenkou_iryou/iryouhoken/iryouhoken15/index.html, last accessed on April 24, $2012 .{ }^{375}$

MHLW, Ministry of Health, Labor and Welfare; NHI, National Health Insurance. 
Table 62. Requirements for Medical Institutions Providing Cardiovascular Rehabilitation

\section{Disease group \\ Cardiovascular rehabilitation (I)}

Physicians

Healthcare professionals

Criteria for medical institutions

$\mathrm{NHI}$ reimbursement 200 points

Maximum duration 150 days

This service should be provided in insurance authorized medica institutions where cardiologists or cardiovascular surgeons are working full time during the period when cardiovascular rehabilitation is provided, and at least one physician who has experience in and is responsible for cardiovascular rehabilitation is working. The medical institutions should have appropriate systems to communicate with the responsible physicians when a sudden change in a patient's condition occurs during rehabilitation, and to treat the patient appropriately in the institution or affiliated NHI-covered institutions.

This service should be provided in medical institutions where a total of $\geq 2$ full-time physical therapists and/or full-time nurses who have experience in and are dedicated to cardiovascular rehabilitation are working. One of the two staff members may be a member who is responsible for the service. The above-mentioned staff members may not work as staff members in the recoveryphase rehabilitation ward, but may provide rehabilitation sessions for patients with other diseases, disabled children/patients, or patients with cancer during the time when they are not involved in cardiovascular rehabilitation. The staff members may also be registered as being dedicated to other types of rehabilitation when different rehabilitation programs are scheduled on different days and times.

The medical institutions should have a room dedicated to functional training with an area of $\geq 30 \mathrm{~m}^{2}$ in hospitals and $\geq 20 \mathrm{~m}^{2}$ in clinics. The room dedicated for functional training may be used for other purposes during the time when the relevant service is not provided. Functional training rooms for cardiovascular rehabilitation may be used as rooms for rehabilitation for patients with other diseases, disabled children/patients or cancer patients, provided that the rooms meet the criteria for relevant rehabilitation rooms. For example, when cardiovascular and cerebrovascular rehabilitation are provided simultaneously, the functional training room should be larger than the sum of the areas specified in the relevant criteria and should be equipped with devices and instruments dedicated for the relevant rehabilitation services.

\section{Rehabilitation during hospitalization is subject to the early-phase \\ Rehabilitation during hospitalization is subject to the early-phas
rehabilitation premium ( 30 points/unit) during the first 30 days,
and the initial phase premium ( 45 points/units) during the first 14 \\ Rehabilitation during hospitalization is subject to the early-phase
rehabilitation premium ( 30 points/unit) during the first 30 days,
and the initial phase premium ( 45 points/units) during the first 14 days of rehabilitation.}

of $\mathrm{NHI}$

\section{Cardiovascular rehabilitation (II)}

This service should be provided in medical institutions where at least one experienced fulltime physician is working (Patients with stable condition may conduct rehabilitation sessions without direct supervision by a physician).

This service should be provided in medical institutions where $\geq 1$ physical therapist or nurse who is experienced in and is dedicated to cardiovascular rehabilitation is working. The above-mentioned staff members dedicated to this service may not work as staff members in the recovery-phase rehabilitation ward, but may provide rehabilitation sessions for patients with other diseases, disabled children/patients, or patients with cancer during the time when they are not involved in cardiovascular rehabilitation. The staff members may also be registered as being dedicated to other types of rehabilitation when different rehabilitation programs are scheduled on different days and times.

Same as on the left.

100 points

Rehabilitation during hospitalization is subject to the early-phase rehabilitation premium

(30 points/unit) during the first 30 days, and the initial phase premium (45 points/units) during the first 14 days of rehabilitation.

150 days

*Direct supervision by a physician is required for patients with high-risk arrhythmias, patients with cardiac dysfunction, and patients with exercise-induced ischemia.

Source: Notifications from the Medical Economics Division, Health Insurance Bureau, MHLW regarding the revision of NHI reimbursement list in 2012. URL: http://www.mhlw.go.jp/seisakunitsuite/bunya/kenkou_iryou/iryouhoken/iryouhoken15/index.html, last accessed on April 24, 2012.375

MHLW, Ministry of Health, Labor and Welfare; NHI, National Health Insurance.

and 45 points of the "initial phase premium". This revision encourages healthcare professionals to start rehabilitation earlier (Figure 17). These premiums are available in "medical institutions where rehabilitation physicians belong", and the definition of "rehabilitation physicians" is discussed in detail in Office Communication of the Medical Economics Division, Health Insurance Bureau, MHLW dated March 30, 2012. Please refer to it. It should be noted that these premiums are applied to rehabilitation services in medical institutions where at least one physician dedicated to rehabilitation is working on a regular basis even if such medical institutions have not notified the prefectural government that they will provide rehabilitation services. Cardiovascular rehabilitation is covered with the NHI premiums in medical institutions proving cardiovascular or cardiovascu- lar surgery services in which at least one full-time physician is experienced in "cardiovascular rehabilitation". Accordingly, hospitals satisfying the criteria for medical institutions providing cardiovascular rehabilitation may claim the above-mentioned NHI premiums.

2. The NHI reimbursement added samples of "cardiovascular rehabilitation protocol for inpatients (Attached Form 214)" (Table 54), 325 "cardiovascular rehabilitation protocol for outpatients (Attached Form 21-5)" (Table 55), 325 and "integrated cardiovascular rehabilitation protocol (Attached Form 23-4)" (Table 56) 326 $^{32}$ to facilitate healthcare professionals in developing cardiovascular rehabilitation programs. 
Table 63. General Remarks for Fees for Cardiovascular Rehabilitation

1. Equipment and system

(1) Rooms dedicated for functional training should be equipped with necessary devices and instruments, i.e., defibrillators, ECG monitors, treadmill or ergometer machines, blood pressure monitors, and emergency carts. Cardiopulmonary exercise testing devices should be available in the hospital providing the service.

(2) Records about rehabilitation sessions (e.g., physician's directions, exercise prescriptions, duration and contents of rehabilitation, and a list of responsible staff members) should be managed in an integrated fashion for each patient and should be accessible by healthcare professionals.

(3) Multidisciplinary case conferences should be held regularly.

(4) The hospital should manage emergency situations occurring during rehabilitation. Specifically, emergency surgery and emergency angiography should be available in the hospital providing the service or affiliated medical institutions providing NHI-covered cardiovascular or cardiovascular surgery services. The hospital providing the service or affiliated medical institutions should be registered as medical institutions that may claim fees for critical care treatment and frees for designated ICU treatment.

2. Standard length of session of cardiovascular rehabilitation and the number of patients per staff member

Rehabilitation sessions should be conducted under instruction and control by a physician responsible for the service. The physician should directly supervise patients, or should be in the hospital and communicable to staff members directly supervising patients and should be able to respond to emergency situations without delay. The length of a standard session is about 1 hour ( 3 units). Outpatients should conduct rehabilitation 3 hours ( 9 units) per week as sessions of $\geq 1$ hour ( 3 units) as a standard menu. The numbers of inpatients supervised by a responsible physician, physical therapist and nurse during a session should be about 15,5 and 5 , respectively, and those of other patients should be about 20,8 and 8 , respectively. When a healthcare professional dedicated to "cardiovascular rehabilitation" provides rehabilitation sessions to patients with cardiovascular diseases and to other patients, he or she may provide a total of 18 service units per day and $\leq 108$ service units per week, where 1 service unit is defined as a 20-minute service in terms of cardiovascular rehabilitation.

3. Fees for examination conducted during rehabilitation should be included in the fees for cardiovascular rehabilitation.

The $\mathrm{NHI}$ reimbursement for cardiovascular rehabilitation include fees for conducting ECG examination, exercise ECG, heart/respiratory rate monitoring, heart/respiratory rate monitoring in newborn infants, cardioscope (heart scope), and cardiotachoscope that are conducted during rehabilitation sessions.

4. The $\mathrm{NHI}$ covers $\leq 13$ units/month of maintenance-phase rehabilitation

For patients in whom continued rehabilitation is considered medically appropriate, the $\mathrm{NHI}$ covers $\leq 13$ units/months of maintenance-phase rehabilitation after the standard maximum duration of $\mathrm{NHI}$-covered rehabilitation.

5. The service may be provided after the standard maximum duration of $\mathrm{NHI}$-covered rehabilitation.

Patients indicated for cardiovascular rehabilitation may continue rehabilitation after the maximum duration of $\mathrm{NHI}$-covered rehabilitation when continued rehabilitation is expected to improve the patient's condition on a medical basis and when appropriate documents containing necessary descriptions are submitted.

ICU, intensive care unit; NHI, National Health Insurance.

\section{The Future of Cardiac Rehabilitation From the Health Economics Viewpoint}

\section{Introduction}

This section discusses the health economic value of cardiac rehabilitation, and provides basic information for further socioeconomic consideration of comprehensive cardiac rehabilitation. Because the benefits of cardiac rehabilitation in micro are discussed in earlier sections, this section mainly discuss cost-effectiveness and benefits of cardiac rehabilitation for macro. This section first describes the health-economic evidence of the benefits of comprehensive cardiac rehabilitation in micro with CAD and other cardiovascular diseases, and then lists cases in foreign countries (although not sufficient to serve as evidence) as examples of the effects of cardiac rehabilitation on the health insurance resources and industries in the society.

\section{Health-Economic Effects of Exercise Training on Micro Class I}

1. Long-term exercise intervention improves well-being of patients (utility value and life years), and reduces healthcare cost. (Level of Evidence: A)

\section{Class Ila}

1. Long-term exercise intervention improves the balance between benefits and burdens (cost-effectiveness) of patients and family members. (Level of Evidence: B)
2. Exercise intervention for less than 12 months improves the well-being of patients (utility value and life years) and reduces healthcare cost. (Level of Evidence: $\mathbf{C}$ )

\section{Class IIb}

1. Exercise intervention for less than 12 months improves the balance between benefits and burdens (cost-effectiveness) of patients and family members. (Level of Evidence: C).

In a study on the health-economic performance of cardiac rehabilitation for 10 years, the cost required to gain an additional life-year is US \$806 among patients undergoing cardiac rehabilitation, which is lower than the US $\$ 1,073$ required for patients without cardiac rehabilitation (Table 64). ${ }^{335}$ In another study where the cost-effectiveness of a 14-month exercise training program in patients with stable chronic heart failure, the cost required to gain an additional life-year was higher in patients with cardiac rehabilitation than in those without it. These findings indicated that longer exercise training is more cost-effective. ${ }^{376}$

In a study of the cost-effectiveness of cardiac rehabilitation in secondary prevention of cardiovascular diseases using the cost-utility analysis, the cost utility was US $\$ 640$ saved per quality-adjusted life-year (QALY) gained, which indicates that cardiac rehabilitation may reduce healthcare costs and improve QALY compared to usual care (without cardiac rehabilitation). ${ }^{377}$ 


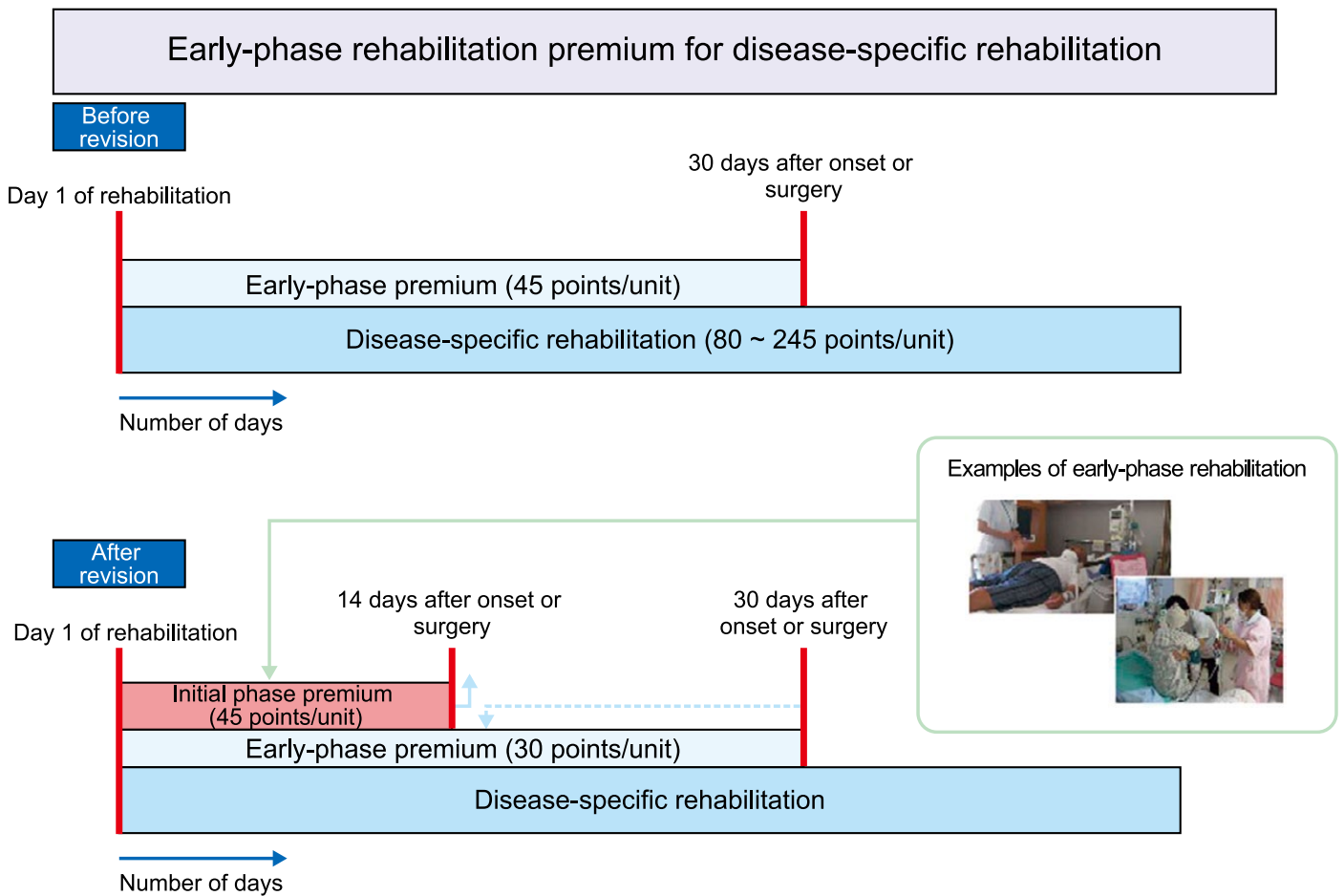

Figure 17. Relationship between the early-phase rehabilitation premium and the initial phase premium for disease-specific rehabilitation.

Table 64. Estimated Cost-Effectiveness of Cardiac Rehabilitation

\section{Groups}

Patients undergoing cardiac rehabilitation

Patients without cardiac rehabilitation

\begin{tabular}{c} 
Study \\
\hline Duration \\
Total cost
\end{tabular}

Additional life-years

(US \$)/life year

Total cost

Additional life-years

(US \$)/life year

\begin{tabular}{ccc} 
Georgiou $(n=99)$ & & Inglis $(n=297)$ \\
\cline { 1 - 1 } months & & 10 years \\
5,282 & & 3,267 \\
10.2 & & 4.1 \\
515.8 & & 806.7 \\
2,055 & & 3,059 \\
8.0 & & 2.9 \\
258.2 & & $1,073.3$
\end{tabular}

Source: Inglis SC, et al. Extending the horizon in chronic heart failure: effects of multidisciplinary, home-based intervention relative to usual care. Circulation 2006; 114: 2466-2473, ${ }^{335}$ and Georgiou D, et al. Cost-effectiveness analysis of long-term moderate exercise training in chronic heart failure. Am J Cardiol 2001; 87: 984-988. ${ }^{376}$

Table 65. Effects of Cardiac Rehabilitation on Patient's Returning to Work

\section{Results}

Returning to work within 3 months

Returning to work after 3 months

Returning to work within 3 months but stopped working within 1 year

Never returning to work

\begin{tabular}{ccc}
\multicolumn{2}{c}{$\%$ returning to work } & \\
\cline { 1 - 2 } $\begin{array}{c}\text { Rehabilitation } \\
\text { participants }(\mathbf{n = 9 6 )}\end{array}$ & $\begin{array}{c}\text { Matched } \\
\text { controls }(\mathbf{n}=156)\end{array}$ & \\
53.1 & 34.8 & \\
10.4 & 7.1 & 0.001 \\
19.9 & 31.0 & \\
16.7 & 27.1 & \\
\hline
\end{tabular}

Source: Simchen E, et al. Is participation in cardiac rehabilitation programs associated with better quality of life and return to work after coronary artery bypass operations? Isr Med Assoc J 2001; 3: 399-403. ${ }^{379}$ 


\section{Health-Economic Benefits of Exercise Training on Macro Class I}

None.

\section{Class Ila}

1. Exercise intervention over a period of time is expected to help patients return to work and increase labor productivity. (Level of Evidence: B)

2. Exercise intervention over a period of time alleviates the financial burden on community healthcare insurance systems and improves the management efficiency of medical institutions. (Level of Evidence: C)

\section{Class IIb}

1. Exercise intervention saves health insurance resources, reduces the financial burden on insurers and society, and improves the economic performance (cost-effectiveness) of the health care system. (Level of Evidence: C)

Studies on the effect of cardiac rehabilitation on the health insurance resources have demonstrated that the healthcare costs for patients undergoing comprehensive cardiac rehabilitation was significantly lower than those for patients without it, and suggested that cardiac rehabilitation may help reduce healthcare costs of the whole country. In a study in the United States, cardiac rehabilitation resulted in a gross cost savings of US $\$ 956$ per patient (US \$159/patient per month) during 6 months, and US \$1,863 per patient during 36 months (US \$52/ patient per month). 378

In another study of the effect of cardiac rehabilitation programs on work productivity in patients after CABG, patients who participated in cardiac rehabilitation programs for 3 6 months returned to work earlier than those without cardiac rehabilitation (the percentage of patients returning to work within 3 months was $53.1 \%$ vs. $34.8 \%$, respectively), and the percentages of patients who were rehospitalized and of those who stopped work after initially going back to work were lower in those with, than in those without, cardiac rehabilitation (Table 65). ${ }^{379}$ Sufficient studies have not been conducted on the effects of cardiac rehabilitation on industries. Further studies such as those using the methods of Input-Output analysis should be performed in the future.

\section{Conclusion}

This abbreviated guidelines contain a list of recommendations with a class of recommendation and a level of evidence in accordance with other guideline documents published by the JCS. Currently, there is insufficient evidence to make recommendations on cardiac rehabilitation worldwide. The lack of evidence is a serious problem, especially in Japan where evidence from multicenter clinical studies is limited and relies on foreign data. The NHI reimbursement for cardiac rehabilitation has been changed almost every year, but the importance of cardiac rehabilitation has not been fully understood or recognized, and the government has not provided a sufficient budget for this area. We hope this guideline will help cardiologists,

\section{References}

1. Itoh H. Chapter 6. Recovery phase rehabilitation. In: Takano T, editor. Acute myocardial infarction, 2nd edn, Book 4 of $\mathrm{ABC}$ for new diagnosis and treatment. Tokyo: Saishin Igaku Sha, 2009; 278-286 (in Japanese).

2. Kouzuki M, Saito M, Iwasaka T, Daida H, Ueshima K, Makita S, et al. Profitability of cardiac rehabilitation in Japan: Results of a multi-center study. Journal of Japanese Association of Cardiac Rehabilitation (JJCR) 2009; 14: 269-275 (in Japanese).

3. Wenger NK, Froelicher ES, Smith LK, Philip A, Ades PA, Berra K, et al. Clinical Practice Guidelines No. 17: Cardiac Rehabilitation as Secondary Prevention. Rockville, MD: U.S. Department of Health and Human Services, Public Health Service, Agency for Health Care Policy and Research, National Heart, Lung and Blood Institute, 1995. AHCPR Publication 96-0672. (C)

4. Belardinelli R, Georgiou D, Cianci G, Purcaro A. Randomized, controlled trial of long-term moderate exercise training in chronic heart failure: Effects on functional capacity, quality of life, and clinical outcome. Circulation 1999; 99: 1173-1182. (A)

5. Oldridge NB, Guyatt GH, Fischer ME, Rimm AA. Cardiac rehabilitation after myocardial infarction. Combined experience of randomized clinical trials. JAMA 1988; 260: 945-950. (A)

6. O'Connor GT, Buring JE, Yusuf S, Goldhaber SZ, Olmstead EM, Paffenbarger RS Jr, et al. An overview of randomized trials of rehabilitation with exercise after myocardial infarction. Circulation 1989; 80: 234-244. (A)

7. European Heart Failure Training Group. Experience from controlled trials of physical training in chronic heart failure: Protocol and patient factors in effectiveness in the improvement in exercise toler- nurses, and other healthcare professionals provide appropriate cardiac rehabilitation programs for their patients. In this revision, we describe in detail the health-economic benefits of cardiac rehabilitation, and the change over time in the coverage of cardiac rehabilitation programs by the national insurance system, to encourage the usage of cardiac rehabilitation in the clinical setting. Because the NHI reimbursement is revised every year and recommendations will become outdated, we are seeking a way to provide up-to-date information. We expect that many healthcare professionals will understand and utilize this guideline document in the clinical setting and lead the advance of cardiac rehabilitation in Japan.

ance. Eur Heart J 1998; 19: 466-475. (A)

8. Pavy B, Iliou MC, Meurin P, Tabet JY, Corone S; Functional Evaluation and Cardiac Rehabilitation Working Group of the French Society of Cardiology. Safety of exercise training for cardiac patients: Results of the French registry of complications during cardiac rehabilitation. Arch Intern Med 2006; 166: 2329-2334. (A)

9. Giannuzzi P, Tavazzi L, Temporelli PL, Corrà U, Imparato A, Gattone $\mathrm{M}$, et al. Long-term physical training and left ventricular remodeling after anterior myocardial infarction: Results of the Exercise in Anterior Myocardial Infarction (EAMI) trial. EAMI Study Group. J Am Coll Cardiol 1993; 22: 1821 - 1829. (A)

10. Giannuzzi P, Temporelli PL, Corrà U, Gattone M, Giordano A, Tavazzi L. Attenuation of unfavorable remodeling by exercise training in postinfarction patients with left ventricular dysfunction: Results of the Exercise in Left Ventricular Dysfunction (ELVD) trial. Circulation 1997; 96: 1790-1797. (A)

11. Hambrecht R, Gielen S, Linke A, Fiehn E, Yu J, Walther C, et al. Effects of exercise training on left ventricular function and peripheral resistance in patients with chronic heart failure: A randomized trial. JAMA 2000; 283: 3095-3101. (A)

12. van Tol BA, Huijsmans RJ, Kroon DW, Schothorst M, Kwakkel G. Effects of exercise training on cardiac performance, exercise capacity and quality of life in patients with heart failure: A meta-analysis. Eur J Heart Fail 2006; 8: 841 -850. (A)

13. Giannuzzi P, Temporelli PL, Corrà U, Tavazzi L; ELVD-CHF Study Group. Antiremodeling effect of long-term exercise training in patients with stable chronic heart failure: Results of the Exercise in Left Ventricular Dysfunction and Chronic Heart Failure (ELVDCHF) Trial. Circulation 2003; 108: 554-559. (A)

14. Marchionni N, Fattirolli F, Fumagalli S, Oldridge N, Del Lungo F, 
Morosi L, et al. Improved exercise tolerance and quality of life with cardiac rehabilitation of older patients after myocardial infarction: Results of a randomized, controlled trial. Circulation 2003; 107: 2201-2206. (A)

15. Sakuragi S, Takagi S, Suzuki S, Sakamaki F, Takaki H, Aihara N, et al. Patients with large myocardial infarction gain a greater improvement in exercise capacity after exercise training than those with small to medium infarction. Clin Cardiol 2003; 26: 280-286.

16. Giallauria F, De Lorenzo A, Pilerci F, Manakos A, Lucci R, Psaroudaki $\mathrm{M}$, et al. Reduction of $\mathrm{N}$ terminal-pro-brain (B-type) natriuretic peptide levels with exercise-based cardiac rehabilitation in patients with left ventricular dysfunction after myocardial infarction. Eur $J$ Cardiovasc Prev Rehabil 2006; 13: 625-632. (B)

17. Yu CM, Li LS, Lam MF, Siu DC, Miu RK, Lau CP. Effect of a cardiac rehabilitation program on left ventricular diastolic function and its relationship to exercise capacity in patients with coronary heart disease: Experience from a randomized, controlled study. Am Heart J 2004; 147: e24, doi:10.1016/j.ahj.2003.12.004. (A)

18. Myers J, Wagner D, Schertler T, Beer M, Luchinger R, Klein M, et al. Effects of exercise training on left ventricular volumes and function in patients with nonischemic cardiomyopathy: Application of magnetic resonance myocardial tagging. Am Heart $J$ 2002; 144: 719-725. (B)

19. Stolen KQ, Kemppainen J, Kalliokoski KK, Luotolahti M, Viljanen $\mathrm{T}$, Nuutila $\mathrm{P}$, et al. Exercise training improves insulin-stimulated myocardial glucose uptake in patients with dilated cardiomyopathy. J Nucl Cardiol 2003; 10: 447-455. (B)

20. Stolen KQ, Kemppainen J, Ukkonen H, Kalliokoski KK, Luotolahti $\mathrm{M}$, Lehikoinen $\mathrm{P}$, et al. Exercise training improves biventricular oxidative metabolism and left ventricular efficiency in patients with dilated cardiomyopathy. J Am Coll Cardiol 2003; 41: 460-467. (B)

21. Sullivan MJ, Higginbotham MB, Cobb FR. Exercise training in patients with severe left ventricular dysfunction: Hemodynamic and metabolic effects. Circulation 1988; 78: 506-515. (A)

22. Detry JM, Rousseau M, Vandenbroucke G, Kusumi F, Brasseur LA, Bruce RA. Increased arteriovenous oxygen difference after physical training in coronary heart disease. Circulation 1971; 44: 109-118. (B)

23. Froelicher V, Jensen D, Genter F, Sullivan M, McKirnan MD, Witztum K, et al. A randomized trial of exercise training in patients with coronary heart disease. JAMA 1984; 252: 1291-1297. (A)

24. Schuler G, Hambrecht R, Schlierf G, Grunze M, Methfessel S, Hauer K, et al. Myocardial perfusion and regression of coronary artery disease in patients on a regimen of intensive physical exercise and low fat diet. J Am Coll Cardiol 1992; 19: 34-42. (A)

25. Ornish D, Scherwitz LW, Billings JH, Brown SE, Gould KL, Merritt TA, et al. Intensive lifestyle changes for reversal of coronary heart disease. JAMA 1998; 280: 2001 -2007. (A)

26. Haskell WL, Alderman EL, Fair JM, Maron DJ, Mackey SF, Superko $\mathrm{HR}$, et al. Effects of intensive multiple risk factor reduction on coronary atherosclerosis and clinical cardiac events in men and women with coronary artery disease: The Stanford Coronary Risk Intervention Project (SCRIP). Circulation 1994; 89: 975-995. (A)

27. Niebauer J, Hambrecht R, Velich T, Hauer K, Marburger C, Kälberer $\mathrm{B}$, et al. Attenuated progression of coronary artery disease after 6 years of multifactorial risk intervention: Role of physical exercise. Circulation 1997; 96: 2534-2541. (A)

28. Ehsani AA, Heath GW, Hagberg JM, Sobel BE, Holloszy JO. Effects of 12 months of intense exercise training on ischemic STsegment depression in patients with coronary artery disease. Circulation 1981; 64: 1116-1124. (B)

29. Hambrecht R, Wolf A, Gielen S, Linke A, Hofer J, Erbs S, et al. Effect of exercise on coronary endothelial function in patients with coronary artery disease. $N$ Engl J Med 2000; 342: 454-460. (A)

30. Coats AJ, Adamopoulos S, Radaelli A, McCance A, Meyer TE, Bernardi L, et al. Controlled trial of physical training in chronic heart failure: Exercise performance, hemodynamics, ventilation, and autonomic function. Circulation 1992; 85: 2119-2131. (B)

31. Piepoli M, Clark AL, Volterrani M, Adamopoulos S, Sleight P, Coats AJ. Contribution of muscle afferents to the hemodynamic, autonomic, and ventilatory responses to exercise in patients with chronic heart failure: Effects of physical training. Circulation 1996; 93: $940-952$. (A)

32. Mancini DM, Henson D, La Manca J, Donchez L, Levine S. Benefit of selective respiratory muscle training on exercise capacity in patients with chronic congestive heart failure. Circulation 1995; 91: 320-329. (A)

33. Sullivan MJ, Higginbotham MB, Cobb FR. Exercise training in patients with chronic heart failure delays ventilatory anaerobic thresh- old and improves submaximal exercise performance. Circulation 1989; 79: 324-329. (A)

34. Kiilavuori K, Sovijärvi A, Näveri H, Ikonen T, Leinonen H. Effect of physical training on exercise capacity and gas exchange in patients with chronic heart failure. Chest 1996; 110: 985-991. (A)

35. Taylor RS, Brown A, Ebrahim S, Jolliffe J, Noorani H, Rees K, et al. Exercise-based rehabilitation for patients with coronary heart disease: Systematic review and meta-analysis of randomized controlled trials. Am J Med 2004; 116: 682-692. (A)

36. Jolliffe JA, Rees K, Taylor RS, Thompson D, Oldridge N, Ebrahim S. Exercise-based rehabilitation for coronary heart disease. Cochrane Database Syst Rev 2001; (1): CD001800. (A)

37. Piepoli MF, Davos C, Francis DP, Coats AJ; ExTraMATCH Collaborative. Exercise training meta-analysis of trials in patients with chronic heart failure (ExTraMATCH). BMJ 2004; 328: 189. (A)

38. Taylor RS, Unal B, Critchley JA, Capewell S. Mortality reductions in patients receiving exercise-based cardiac rehabilitation: How much can be attributed to cardiovascular risk factor improvements? Eur J Cardiovasc Prev Rehabil 2006; 13: 369-374. (A)

39. Haykowsky MJ, Liang Y, Pechter D, Jones LW, McAlister FA, Clark AM. A meta-analysis of the effect of exercise training on left ventricular remodeling in heart failure patients: The benefit depends on the type of training performed. J Am Coll Cardiol 2007; 49: 2329-2336. (A)

40. Wisløff U, Støylen A, Loennechen JP, Bruvold M, Rognmo Ø, Haram PM, et al. Superior cardiovascular effect of aerobic interval training versus moderate continuous training in heart failure patients: A randomized study. Circulation 2007; 115: 3086-3094. (B)

41. Beer M, Wagner D, Myers J, Sandstede J, Köstler H, Hahn D, et al. Effects of exercise training on myocardial energy metabolism and ventricular function assessed by quantitative phosphorus-31 magnetic resonance spectroscopy and magnetic resonance imaging in dilated cardiomyopathy. J Am Coll Cardiol 2008; 51: 1883-1891.

42. Maiorana A, O'Driscoll G, Dembo L, Cheetham C, Goodman C, Taylor R, et al. Effect of aerobic and resistance exercise training on vascular function in heart failure. Am J Physiol Heart Circ Physiol 2000; 279: H1999-H2005.

43. Hambrecht R, Fiehn E, Weigl C, Gielen S, Hamann C, Kaiser R, et al. Regular physical exercise corrects endothelial dysfunction and improves exercise capacity in patients with chronic heart failure. Circulation 1998; 98: 2709-2715. (A)

44. Adams V, Linke A, Kränkel N, Erbs S, Gielen S, Möbius-Winkler $\mathrm{S}$, et al. Impact of regular physical activity on the NAD(P)H oxidase and angiotensin receptor system in patients with coronary artery disease. Circulation 2005; 111: 555-562. (B)

45. Kingwell BA. Nitric oxide-mediated metabolic regulation during exercise: Effects of training in health and cardiovascular disease. FASEB J 2000; 14: 1685-1696. (C)

46. Kasapis $\mathrm{C}$, Thompson PD. The effects of physical activity on serum C-reactive protein and inflammatory markers: A systematic review. $J$ Am Coll Cardiol 2005; 45: 1563-1569. (B)

47. Smith JK, Dykes R, Douglas JE, Krishnaswamy G, Berk S. Longterm exercise and atherogenic activity of blood mononuclear cells in persons at risk of developing ischemic heart disease. JAMA 1999; 281: $1722-1727$. (B)

48. Gielen S, Adams V, Möbius-Winkler S, Linke A, Erbs S, Yu J, et al. Anti-inflammatory effects of exercise training in the skeletal muscle of patients with chronic heart failure. $J$ Am Coll Cardiol 2003; 42: 861-868. (B)

49. Ingjer F. Effects of endurance training on muscle fibre ATP-ase activity, capillary supply and mitochondrial content in man. $J$ Physiol 1979; 294: 419-432. (B)

50. Hambrecht R, Fiehn E, Yu J, Niebauer J, Weigl C, Hilbrich L, et al. Effects of endurance training on mitochondrial ultrastructure and fiber type distribution in skeletal muscle of patients with stable chronic heart failure. J Am Coll Cardiol 1997; 29: 1067-1073. (B)

51. Tjønna AE, Lee SJ, Rognmo Ø, Stølen TO, Bye A, Haram PM, et al. Aerobic interval training versus continuous moderate exercise as a treatment for the metabolic syndrome: A pilot study. Circulation 2008; 118: 346-354.

52. Iellamo F, Legramante JM, Massaro M, Raimondi G, Galante A. Effects of a residential exercise training on baroreflex sensitivity and heart rate variability in patients with coronary artery disease: A randomized, controlled study. Circulation 2000; 102: 2588-2592. (A)

53. Gademan MG, Swenne CA, Verwey HF, van der Laarse A, Maan $\mathrm{AC}$, van de Vooren $\mathrm{H}$, et al. Effect of exercise training on autonomic derangement and neurohumoral activation in chronic heart failure. J Card Fail 2007; 13: 294-303. (A)

54. Wang JS, Jen CJ, Chen HI. Effects of exercise training and decon- 
ditioning on platelet function in men. Arterioscler Thromb Vasc Biol 1995; 15: $1668-1674$. (A)

55. Suzuki T, Yamauchi K, Yamada Y, Furumichi T, Furui H, Tsuzuki $\mathrm{J}$, et al. Blood coagulability and fibrinolytic activity before and after physical training during the recovery phase of acute myocardial infarction. Clin Cardiol 1992; 15: 358-364. (A)

56. Lee KW, Blann AD, Jolly K, Lip GY; BRUM Investigators. Plasma haemostatic markers, endothelial function and ambulatory blood pressure changes with home versus hospital cardiac rehabilitation: The Birmingham Rehabilitation Uptake Maximisation Study. Heart 2006; 92: 1732 - 1738. (B)

57. Hambrecht R, Walther C, Möbius-Winkler S, Gielen S, Linke A, Conradi K, et al. Percutaneous coronary angioplasty compared with exercise training in patients with stable coronary artery disease: A randomized trial. Circulation 2004; 109: 1371-1378. (B)

58. Conraads VM, Beckers P, Vaes J, Martin M, Van Hoof V, De Maeyer C, et al. Combined endurance/resistance training reduces NT-proBNP levels in patients with chronic heart failure. Eur Heart J 2004; 25: 1797-1805.

59. Balady GJ, Williams MA, Ades PA, Bittner V, Comoss P, Foody $\mathrm{JM}$, et al. Core components of cardiac rehabilitation/secondary prevention programs: 2007 update: A scientific statement from the American Heart Association Exercise, Cardiac Rehabilitation, and Prevention Committee, the Council on Clinical Cardiology; the Councils on Cardiovascular Nursing, Epidemiology and Prevention, and Nutrition, Physical Activity, and Metabolism; and the American Association of Cardiovascular and Pulmonary Rehabilitation. Circulation 2007; 115: 2675-2682. (C)

60. Aragam KG, Moscucci M, Smith DE, Riba AL, Zainea M, Chambers $\mathrm{JL}$, et al. Trends and disparities in referral to cardiac rehabilitation after percutaneous coronary intervention. Am Heart J 2011; 161: 544-551.e2.

61. Day W. Women and cardiac rehabilitation: A review of the literature. Contemp Nurse 2003-2004; 16: 92-101.

62. Smith SC Jr, Allen J, Blair SN, Bonow RO, Brass LM, Fonarow $\mathrm{GC}$, et al. AHA/ACC guidelines for secondary prevention for patients with coronary and other atherosclerotic vascular disease: 2006 update: Endorsed by the National Heart, Lung, and Blood Institute. Circulation 2006; 113: 2363-2372.

63. Beckie TM, Beckstead JW. Predicting cardiac rehabilitation attendance in a gender-tailored randomized clinical trial. $J$ Cardiopulm Rehabil Prev 2010; 30: 147-156.

64. Mallik S, Spertus JA, Reid KJ, Krumholz HM, Rumsfeld JS, Weintraub WS, et al; PREMIER Registry Investigators. Depressive symptoms after acute myocardial infarction: Evidence for highest rates in younger women. Arch Intern Med 2006; 166: 876-883.

65. Gottlieb SS, Kop WJ, Ellis SJ, Binkley P, Howlett J, O'Connor C, et al; HF-ACTION Investigators. Relation of depression to severity of illness in heart failure (from Heart Failure And a Controlled Trial Investigating Outcomes of Exercise Training [HF-ACTION]). Am J Cardiol 2009; 103: 1285-1289. (C)

66. Gilson BS, Gilson JS, Bergner M, Bobbit RA, Kressel S, Pollard WE, et al. The sickness impact profile: Development of an outcome measure of health care. Am J Public Health 1975; 65: 1304-1310. (C)

67. Chambers LW, Macdonald LA, Tugwell P, Buchanan WW, Kraag G. The McMaster Health Index Questionnaire as a measure of quality of life for patients with rheumatoid disease. J Rheumatol 1982; 9: 780-784. (C)

68. Hunt SM, McKenna SP, McEwen J, Backett EM, Williams J, Papp E. A quantitative approach to perceived health status: A validation study. J Epidemiol Community Health 1980; 34: 281 -286. (C)

69. Ware JE Jr, Sherbourne CD. The MOS 36-item short-form health survey (SF-36). I: Conceptual framework and item selection. Med Care 1992; 30: 473-483. (C)

70. Rector T, Kubo S, Cohn J. Patients'self-assessment of their congestive heart failure. Part 2: Content, reliability and validity of a new measure: The Minnesota Living with Heart Failure Questionnaire. Heart Failure 1987; 3: 198-209. (C)

71. Green CP, Porter CB, Bresnahan DR, Spertus JA. Development and evaluation of the Kansas City Cardiomyopathy Questionnaire: A new health status measure for heart failure. J Am Coll Cardiol 2000; 35: $1245-1255$. (C)

72. Tamura M, Omiya K, Yamada S, Oka K, Suzuki N, Osada N, et al. Development of measure for disease-specific quality of life in patients with chronic heart failure. J Cardiol 2003; 42: 155-164 (in Japanese). (C)

73. Moholdt TT, Amundsen BH, Rustad LA, Wahba A, Løvø KT, Gullikstad LR, et al. Aerobic interval training versus continuous moderate exercise after coronary artery bypass surgery: A random- ized study of cardiovascular effects and quality of life. Am Heart $J$ 2009; 158: 1031-1037. (A)

74. Nilsson BB, Westheim A, Risberg MA. Long-term effects of a groupbased high-intensity aerobic interval-training program in patients with chronic heart failure. Am J Cardiol 2008; 102: 1220-1224. (A)

75. Nilsson BB, Westheim A, Risberg MA. Effects of group-based high-intensity aerobic interval training in patients with chronic heart failure. Am J Cardiol 2008; 102: 1361 - 1365. (A)

76. Karavidas A, Parissis J, Arapi S, Farmakis D, Korres D, Nikolaou $\mathrm{M}$, et al. Effects of functional electrical stimulation on quality of life and emotional stress in patients with chronic heart failure secondary to ischaemic or idiopathic dilated cardiomyopathy: A randomised, placebo-controlled trial. Eur J Heart Fail 2008; 10: 709-713. (A)

77. May GS, Eberlein KA, Furberg CD, Passamani ER, DeMets DL. Secondary prevention after myocardial infarction: A review of longterm trials. Prog Cardiovasc Dis 1982; 24: 331-352. (A)

78. Leon AS, Franklin BA, Costa F, Balady GJ, Berra KA, Stewart KJ, et al. Cardiac rehabilitation and secondary prevention of coronary heart disease: An American Heart Association scientific statement from the Council on Clinical Cardiology (Subcommittee on Exercise, Cardiac Rehabilitation, and Prevention) and the Council on Nutrition, Physical Activity, and Metabolism (Subcommittee on Physical Activity), in collaboration with the American association of Cardiovascular and Pulmonary Rehabilitation. Circulation 2005; 111: $369-376 .(\mathrm{C})$

79. Dorn J, Naughton J, Imamura D, Trevisan M. Results of a multicenter randomized clinical trial of exercise and long-term survival in myocardial infarction patients: The National Exercise and Heart Disease Project (NEHDP). Circulation 1999; 100: 1764-1769. (A)

80. Merz CN, Rozanski A, Forrester JS. The secondary prevention of coronary artery disease. Am J Med 1997; 102: 572-581. (C)

81. Church TS, Lavie CJ, Milani RV, Kirby GS. Improvements in blood rheology after cardiac rehabilitation and exercise training in patients with coronary heart disease. Am Heart J 2002; 143: 349-355. (B)

82. Rauramaa R, Li G, Väisänen SB. Dose-response and coagulation and hemostatic factors. Med Sci Sports Exerc 2001; 33(6 Suppl): S516-S520; discussion S528-S529. (B)

83. Sakuragi S, Takagi S, Suzuki S, Sakamaki F, Aihara N, Yasumura $\mathrm{Y}$, et al. Improvement in PAI-1 levels in blood of patients with acute myocardial infarction during recovery-phase cardiac rehabilitation and its mechanism. Journal of Japanese Association of Cardiac Rehabilitation (JJCR) 2003; 8: 165-170 (in Japanese). (B)

84. Milani RV, Lavie CJ, Mehra MR. Reduction in C-reactive protein through cardiac rehabilitation and exercise training. $J$ Am Coll Cardiol 2004; 43: 1056-1061. (B)

85. Wallner S, Watzinger N, Lindschinger M, Smolle KH, Toplak H, Eber B, et al. Effects of intensified lifestyle modification on the need for further revascularization after coronary angioplasty. Eur $J$ Clin Invest 1999; 29: 372-379. (A)

86. Japanese Society of Hypertension Subcommittee for the Management of Hypertension. Guidelines for the Management of Hypertension (JSH 2004). Tokyo: Life Science Publication, 2004 (in Japanese ). (C)

87. Sacks FM, Pfeffer MA, Moye LA, Rouleau JL, Rutherford JD, Cole TG, et al. The effect of pravastatin on coronary events after myocardial infarction in patients with average cholesterol levels: Cholesterol and Recurrent Events Trial investigators. $N$ Engl J Med 1996; 335: 1001-1009. (A)

88. Pyŏrälä K, Pedersen TR, Kjekshus J, Faergeman O, Olsson AG, Thorgeirsson G. Cholesterol lowering with simvastatin improves prognosis of diabetic patients with coronary heart disease: A subgroup analysis of the Scandinavian Simvastatin Survival Study (4S). Diabetes Care 1997; 20: 614-620. (A)

89. Dinnes J, Kleijnen J, Leitner M, Thompson D. Cardiac rehabilitation. Qual Health Care 1999; 8: 65-71. (C)

90. The Japanese Association of Cardiac Rehabilitation. Cardiac rehabilitation (AHCPR guidelines). Tokyo: Kyowa Kikaku, 1996: 6373 (in Japanese). (C)

91. Detry JR, Vierendeel IA, Vanbutsele RJ, Robert AR. Early shortterm intensive cardiac rehabilitation induces positive results as long as one year after the acute coronary event: A prospective one-year controlled study. J Cardiovasc Risk 2001; 8: 355-361. (B)

92. Guidelines for the Diagnosis and Treatment of Cardiovascular Diseases (1999-2000 Joint Working Groups Report). Guidelines for the Primary Prevention of Ischemic Heart Disease. Jpn Circ J 2001; 65(Suppl V): 999-1065 (in Japanese). (C)

93. Guidelines for the Diagnosis and Treatment of Cardiovascular 
Diseases (2010 Joint Working Groups Report). Guidelines for Secondary Prevention of Myocardial Infarction (JCS2011). http://www. j-circ.or.jp/guideline/pdf/JCS2011_ogawah_h.pdf (in Japanese).

94. Japanese Society of Hypertension Committee. The Japanese Society of Hypertension Guidelines for the Management of Hypertension (JSH 2009) (in Japanese).

95. Japan Atherosclerosis Society. Japan Atherosclerosis Society (JAS) Guidelines for Prevention of Atherosclerotic Cardiovascular Diseases 2012. Tokyo: Kyorinsha, 2012 (in Japanese). (C)

96. Japan Medical Association. Exercise training prescription manual. Japan Medical Association Journal 1996; 116 (Suppl): 1-49 (in Japanese). (C)

97. American College of Sports Medicine. Major signs and symptoms suggestive of cardiovascular and pulmonary disease. ACSM's Guidelines for Exercise Testing and Prescription, 7th edn. Philadelphia: Lippincott Williams \& Wilkins, 2005. (C)

98. Gibbons RJ, Balady GJ, Beasley JW, Bricker JT, Duvernoy WF, Froelicher VF, et al. ACC/AHA Guidelines for Exercise Testing. A report of the American College of Cardiology/American Heart Association Task Force on Practice Guidelines (Committee on Exercise Testing). J Am Coll Cardiol 1997; 30: 260-311.

99. Fletcher GF, Balady GJ, Amsterdam EA, Chaitman B, Eckel R, Fleg J, et al. Exercise standards for testing and training: A statement for healthcare professionals from the American Heart Association. Circulation 2001; 104: 1694-1740. (C)

100. Grundy SM, Brewer HB Jr, Cleeman JI, Smith SC Jr, Lenfant C. Definition of metabolic syndrome: Report of the National Heart, Lung, and Blood Institute/American Heart Association conference on scientific issues related to definition. Circulation 2004; 109: 433-438. (C)

101. Thompson PD, Buchner D, Pina IL, Balady GJ, Williams MA, Marcus BH, et al. Exercise and physical activity in the prevention and treatment of atherosclerotic cardiovascular disease: A statement from the Council on Clinical Cardiology (Subcommittee on Exercise, Rehabilitation, and Prevention) and the Council on Nutrition, Physical Activity, and Metabolism (Subcommittee on Physical Activity). Circulation 2003; 107: 3109-3116. (C)

102. Office for Lifestyle-Related Disease Control, Ministry of Health, Labor and Welfare. Exercise Physical Activity Reference for Health Prormotion 2006 (EPAR 2006): Physical Activity, Exercise and Physical Fitness. http://www0.nih.go.jp/eiken/programs/pdf/epar2006. pdf. (C)

103. Office for Lifestyle-Related Diseases Control, Ministry of Health, Labor and Welfare. Exercise and Physical Activity Guide for Health Promotion $2006 \sim$ To Prevent Lifestyle-related Diseases $<$ Exercise and Physical Activity Guide for Health Program 2006>. http://www0.nih.go.jp/eiken/programs/pdf/exercise_guide.pdf. (C)

104. Borg GA. Perceived exertion. Exerc Sport Sci Rev 1974; 2: 131153.

105. American College of Sports Medicine. ACSM's Guidelines for Exercise Testing and Prescription, 8th edn. Philadelphia: Lippincott Williams \& Wilkins, 2009: 157

106. Cardiac rehabilitation programs. A statement for healthcare professionals from the American Heart Association. Circulation 1994; 90: $1602-1610$. (C)

107. Ades PA. Cardiac rehabilitation and secondary prevention of coronary heart disease. $N$ Engl J Med 2001; 345: 892-902.

108. Saito M, Goto Y, editors. Rehabilitation for angina pectoris and myocardial infarction, 4th edn. Tokyo: Nankodo, 2009 (in Japanese).

109. Cannon CP, Hand MH, Bahr R, Boden WE, Christenson R, Gibler WB, et al; National Heart Attack Alert Program (NHAAP) Coordinating Committee Critical Pathways Writing Group. Critical pathways for management of patients with acute coronary syndromes: An assessment by the National Heart Attack Alert Program. Am Heart J 2002; 143: 777-789.

110. Nakanishi M, Nagayama M, Adachi H, Ikeda K, Fujimoto K, Tashiro T, et al. Prevalence of cardiac rehabilitation after acute myocardial infarction in Japan: A nationwide survey. Journal of Japanese Association of Cardiac Rehabilitation (JJCR) 2001; 16: 188-192 (in Japanese).

111. Goto Y, Noguchi T, Kawakami R, Nakanishi M, Ibuki M, Otuska $\mathrm{Y}$, et al. Regional healthcare information network path for acute myocardial infarction; A perspective based on the results of a nation-wide survey. Heart 2009; 41: 1205-1215 (in Japanese).

112. Anderson JL, Adams CD, Antman EM, Bridges CR, Califf RM, Casey DE Jr, et al. ACC/AHA 2007 Guidelines for the Management of Patients With Unstable Angina/Non-ST-Elevation Myocardial Infarction: Executive Summary. A Report of the American College of Cardiology/American Heart Association Task Force on Practice Guidelines (Writing Committee to Revise the 2002 Guide- lines for the Management of Patients With Unstable Angina/NonST-Elevation Myocardial Infarction). Circulation 2007; 116: $803-$ 877.

113. Pavia L, Orlando G, Myers J, Maestri M, Rusconi C. The effect of beta-blockade therapy on the response to exercise training in postmyocardial infarction patients. Clin Cardiol 1995; 18: 716-720.

114. Malfatto G, Facchini M, Sala L, Branzi G, Bragato R, Leonetti G. Effects of cardiac rehabilitation and beta-blocker therapy on heart rate variability after first acute myocardial infarction. Am J Cardiol 1998; 81: 834-840. (B)

115. Goto Y, Saito M, Iwasaka T, Daida H, Kohzuki M, Ueshima K, et al; Japanese Cardiac Rehabilitation Survey Investigators. Poor implementation of cardiac rehabilitation despite broad dissemination of coronary interventions for acute myocardial infarction in Japan. Circ J 2007; 71: 173-179.

116. Goto Y. Treatment practice: Cardiac rehabilitation. Rinsho to Kenkyu 2011; 88: 1124-1130 (in Japanese).

117. Takahashi T, Sakurada H, Kumamaru M, Saito M, Hanabusa Y, Iwatsu K, et al. Assessment of milestones in rehabilitation after cardiovascular surgery. Journal of Japanese Association of Cardiac Rehabilitation (JJCR) 2012; 17: 103 - 109 (in Japanese).

118. Ferraris VA, Ferraris SP, Moritz DM, Welch S. Oropharyngeal dysphagia after cardiac operations. Ann Thorac Surg 2001; 71: 1792-1795; discussion 1796.

119. Barker J, Martino R, Reichardt B, Hickey EJ, Ralph-Edwards A. Incidence and impact of dysphagia in patients receiving prolonged endotracheal intubation after cardiac surgery. Can J Surg 2009; 52: $119-124$

120. Takahashi T, Jenkins S, Adachi H, Kumamaru M, Sakurai S, Ooshima S, et al. The effectiveness of early chest psysiotherapy for patients after coronary artery bypass graft surgery. Physical Therapy Japan 2011; 28: 31-37(in Japanese). (B)

121. Takahashi T, Nara I, Arizono S, Kumamaru M, Ogasawara MS, Adachi H, et al. Recovery of vital capacity after cardiac surgery: Recovery trend and the effectiveness of incentive spirometry. Physical Therapy Japan 2003; 30: 335-342 (in Japanese).

122. Pasquina $P$, Tramèr MR, Walder B. Prophylactic respiratory physiotherapy after cardiac surgery: Systematic review. BMJ 2003; 327: 1379.

123. Morotomi N, Saito M, Nagayama M, Shimokawa T, Takanashi S. Effect of chest belts on respiratory function in patients after cardiac surgery. Journal of Japanese Association of Cardiac Rehabilitation (JCCR) 2006; Suppl S70 (in Japanese). (B)

124. Pollock ML, Franklin BA, Balady GJ, Chaitman BL, Fleg JL, Fletcher B, et al. AHA Science Advisory. Resistance exercise in individuals with and without cardiovascular disease: Bbenefits, rationale, safety, and prescription: An advisory from the Committee on Exercise, Rehabilitation, and Prevention, Council on Clinical Cardiology, American Heart Association; Position paper endorsed by the American College of Sports Medicine. Circulation 2000; 101: $828-833$. (C)

125. Hedbäck B, Perk J, Hörnblad M, Ohlsson U. Cardiac rehabilitation after coronary artery bypass surgery: 10-year results on mortality, morbidity and readmissions to hospital. J Cardiovasc Risk 2001; 8: $153-158$. (A)

126. Hogue CW Jr, Barzilai B, Pieper KS, Coombs LP, DeLong ER, Kouchoukos NT, et al. Sex differences in neurological outcomes and mortality after cardiac surgery: A society of thoracic surgery national database report. Circulation 2001; 103: 2133-2137.

127. Vaccarino V, Abramson JL, Veledar E, Weintraub WS. Sex differences in hospital mortality after coronary artery bypass surgery: Evidence for a higher mortality in younger women. Circulation 2002; 105: 1176-1181.

128. Zitser-Gurevich Y, Simchen E, Galai N, Mandel M; ISCAB Consortium. Effect of perioperative complications on excess mortality among women after coronary artery bypass: The Israeli Coronary Artery Bypass Graft Study (ISCAB). J Thorac Cardiovasc Surg 2002; 123: 517-524.

129. Woods SE, Noble G, Smith JM, Hasselfeld K. The influence of gender in patients undergoing coronary artery bypass graft surgery: An eight-year prospective hospitalized cohort study. J Am Coll Surg 2003; 196: $428-434$

130. Blankstein R, Ward RP, Arnsdorf M, Jones B, Lou YB, Pine M. Female gender is an independent predictor of operative mortality after coronary artery bypass graft surgery: Contemporary analysis of 31 Midwestern hospitals. Circulation 2005; 112(9 Suppl): I323I327.

131. Hassan A, Chiasson M, Buth K, Hirsch G. Women have worse long-term outcomes after coronary artery bypass grafting than men. Can J Cardiol 2005; 21: 757-762. 
132. Kim C, Redberg RF, Pavlic T, Eagle KA. A systematic review of gender differences in mortality after coronary artery bypass graft surgery and percutaneous coronary interventions. Clin Cardiol 2007; 30: $491-495$.

133. Bukkapatnam RN, Yeo KK, Li Z, Amsterdam EA. Operative mortality in women and men undergoing coronary artery bypass grafting (from the California Coronary Artery Bypass Grafting Outcomes Reporting Program). Am J Cardiol 2010; 105: 339-342.

134. Takagi H, Manabe H, Umemoto T. A contemporary meta-analysis of gender differences in mortality after coronary artery bypass grafting. Am J Cardiol 2010; 106: 1367.

135. Ishii A, Masuda T, Koike T, Saito M, Yonezawa R, Kutsuna T, et al. Assessment of deviation factors in in-hospital rehabilitation after coronary artery bypass grafting. Journal of Japanese Association of Cardiac Rehabilitation (JCCR) 2007; 12: 133-136 (in Japanese).

136. van Dixhoorn J, White A. Relaxation therapy for rehabilitation and prevention in ischaemic heart disease: A systematic review and meta-analysis. Eur J Cardiovasc Prev Rehabil 2005; 12: 193-202. (A)

137. Belardinelli R, Paolini I, Cianci G, Piva R, Georgiou D, Purcaro A Exercise training intervention after coronary angioplasty: The ETICA trial. J Am Coll Cardiol 2001; 37: 1891-1900. (B)

138. Gould KL, Ornish D, Scherwitz L, Brown S, Edens RP, Hess MJ, et al. Changes in myocardial perfusion abnormalities by positron emission tomography after long-term, intense risk factor modification. JAMA 1995; 274: 894-901. (A)

139. The Japanese Association of Cardiac Rehabilitation. Cardiac rehabilitation (AHCPR guidelines). Tokyo: Kyowa Kikaku, 1996 (in Japanese). (C)

140. Goto Y. Rehabilitation for patients undergoing coronary stenting for acute myocardial infarction. Journal of Cardiology 2000 (proceeding): 92 (in Japanese). (C)

141. Goto Y, Sumida H, Ueshima K, Adachi H, Nohara R, Itoh H. Safety and implementation of exercise testing and training after coronary stenting in patients with acute myocardial infarction. Circ J 2002; 66: 930-936. (C)

142. Soga Y, Yokoi H, Kanzaki R, Kimura T, Noda Y, Taniguchi N. Safety of exercise training at anaerobic threshold from the day after elective coronary stenting. Journal of Japanese Association of Cardiac Rehabilitation (JCCR) 2006; 11: 86-89 (in Japanese). (C)

143. Soga Y, Yokoi H, Ando K, Shirai S, Sakai K, Kondo K, et al. Safety of early exercise training after elective coronary stenting in patients with stable coronary artery disease. Eur J Cardiovasc Prev Rehabil 2010; 17: 230-234. (B)

144. Zwart B, Van Kerkvoorde TC, van Werkum JW, Breet NJ, Ten Berg JM, Van't Hof AW. Vigorous exercise as a triggering mechanism for late stent thrombosis: A description of three cases. Platelets 2010; 21: 72-76. (C)

145. Wenger NK. Current status of cardiac rehabilitation. J Am Coll Cardiol 2008; 51: 1619-1631. (C)

146. Fletcher GF, Balady G, Froelicher VF, Hartley LH, Haskell WL, Pollock ML. Exercise standards: A statement for healthcare professionals from the American Heart Association: Writing Group. Circulation 1995; 91: 580-615. (C)

147. Agostoni $\mathrm{P}$, Emdin M, Corrà U, Veglia F, Magrì D, Tedesco CC, et al. Permanent atrial fibrillation affects exercise capacity in chronic heart failure patients. Eur Heart J 2008; 29: 2367-2372.

148. Takahashi N, Ishibashi Y, Shimada T, Sakane T, Ohata S, Sugamori $\mathrm{T}$, et al. Impaired exercise-induced vasodilatation in chronic atrial fibrillation: Role of endothelium-derived nitric oxide. Circ J 2002; 66: $583-588$.

149. Mertens DJ, Kavanagh T. Exercise training for patients with chronic atrial fibrillation. J Cardiopulm Rehabil 1996; 16: 193-196.

150. Vanhees L, Schepers D, Defoor J, Brusselle S, Tchursh N, Fagard R. Exercise performance and training in cardiac patients with atrial fibrillation. J Cardiopulm Rehabil 2000; 20: 346-352.

151. Hegbom F, Sire S, Heldal M, Orning OM, Stavem K, Gjesdal K. Short-term exercise training in patients with chronic atrial fibrillation: Effects on exercise capacity, AV conduction, and quality of life. J Cardiopulm Rehabil 2006; 26: 24-29.

152. Hegbom F, Stavem K, Sire S, Heldal M, Orning OM, Gjesdal K. Effects of short-term exercise training on symptoms and quality of life in patients with chronic atrial fibrillation. Int J Cardiol 2007; 116: $86-92$.

153. Herdy AH, Marcchi PL, Vila A, Tavares C, Collaço J, Niebauer J, et al. Pre- and postoperative cardiopulmonary rehabilitation in hospitalized patients undergoing coronary artery bypass surgery: A randomized controlled trial. Am J Phys Med Rehabil 2008; 87: $714-719$.

154. Ujeyl A, Stevenson LW, West EK, Kato M, Liszkowski M, Campbell
$\mathrm{P}$, et al. Impaired heart rate responses and exercise capacity in heart failure patients with paced baseline rhythms. J Card Fail 2011; 17: $188-195$

155. Wonisch M, Lercher P, Scherr D, Maier R, Pokan R, Hofmann P, et al. Influence of permanent right ventricular pacing on cardiorespiratory exercise parameters in chronic heart failure patients with implanted cardioverter defibrillators. Chest 2005; 127: 787-793.

156. Greco EM, Guardini S, Citelli L. Cardiac rehabilitation in patients with rate responsive pacemakers. Pacing Clin Electrophysiol 1998; 21: $568-575$

157. American College of Sports Medicine. ACSM's Guidelines for Exercise Testing and Prescription, 6th edn. Philadelphia: Lippincott Williams \& Wilkins; 2000. (C)

158. Mead WF, Pyfer HR, Thrombold JC, Frederick RC. Successful resuscitation of two near simultaneous cases of cardiac arrest with a review of fifteen cases occurring during supervised exercise. Circulation 1976; 53: 187-189. (C)

159. Haskel WL. Design and implementation of cardiac conditioning programs. In: Wenger NK, Hellerstein HK, editors. Rehabilitation of the coronary patient. New York: John Wiley \& Sons, 1978: 203-241. (C)

160. Imai S, Nohara R, Ishihara S. Safety of cardiac exercise therapy in the clinical setting. Japanese Journal of Exercise Therapy 2000; 2: 23-27 (in Japanese). (C)

161. Galante A, Pietroiusti A, Cavazzini C, Magrini A, Bergamaschi A, Sciarra L, et al. Incidence and risk factors associated with cardiac arrhythmias during rehabilitation after coronary artery bypass surgery. Arch Phys Med Rehabil 2000; 81: 947-952. (C)

162. Ogasawara R, Ueshima K, Sato S, Saito K, Sotokubo E, Saito M, et al. Arrhythmias newly provoked by exercise training in patients who underwent cardiac surgery. Kyobu Geka 2003; 56: 836-840 (in Japanese).

163. Piña IL, Apstein CS, Balady GJ, Belardinelli R, Chaitman BR, Duscha BD, et al; American Heart Association Committee on exercise, rehabilitation, and prevention. Exercise and heart failure: A statement from the American Heart Association Committee on exercise, rehabilitation, and prevention. Circulation 2003; 107: $1210-$ 1225. (C)

164. Jetté M, Heller R, Landry F, Blümchen G. Randomized 4-week exercise program in patients with impaired left ventricular function. Circulation 1991; 84: 1561 - 1567. (II)

165. Adamopoulos S, Coats AJ, Brunotte F, Arnolda L, Meyer T, Thompson $\mathrm{CH}$, et al. Physical training improves skeletal muscle metabolism in patients with chronic heart failure. J Am Coll Cardiol 1993; 21: 1101 -1106. (IV)

166. Hambrecht R, Niebauer J, Fiehn E, Kälberer B, Offner B, Hauer K, et al. Physical training in patients with stable chronic heart failure: Effects on cardiorespiratory fitness and ultrastructural abnormalities of leg muscles. J Am Coll Cardiol 1995; 25: 1239-1249. (II)

167. Belardinelli R, Georgiou D, Scocco V, Barstow TJ, Purcaro A. Low intensity exercise training in patients with chronic heart failure. $J$ Am Coll Cardiol 1995; 26: 975-982. (IV)

168. Belardinelli R, Georgiou D, Cianci G, Berman N, Ginzton L, Purcaro A. Exercise training improves left ventricular diastolic filling in patients with dilated cardiomyopathy: Clinical and prognostic implications. Circulation 1995; 91: 2775-2784. (II)

169. Kavanagh T, Myers MG, Baigrie RS, Mertens DJ, Sawyer P, Shephard RJ. Quality of life and cardiorespiratory function in chronic heart failure: Effects of 12 months' aerobic training. Heart 1996; 76: $42-49$. (IV)

170. Demopoulos L, Bijou R, Fergus I, Jones M, Strom J, LeJemtel TH. Exercise training in patients with severe congestive heart failure: Enhancing peak aerobic capacity while minimizing the increase in ventricular wall stress. J Am Coll Cardiol 1997; 29: 597-603. (V)

171. Belardinelli R, Georgiou D, Ginzton L, Cianci G, Purcaro A. Effects of moderate exercise training on thallium uptake and contractile response to low-dose dobutamine of dysfunctional myocardium in patients with ischemic cardiomyopathy. Circulation 1998; 97: 553-561. (II)

172. Davies EJ, Moxham T, Rees K, Singh S, Coats AJ, Ebrahim S, et al. Exercise training for systolic heart failure: Cochrane systematic review and meta-analysis. Eur J Heart Fail 2010; 12: 706-715.

173. Smart N, Marwick TH. Exercise training for patients with heart failure: A systematic review of factors that improve mortality and morbidity. Am J Med 2004; 116: 693-706.

174. O'Connor CM, Whellan DJ, Lee KL, Keteyian SJ, Cooper LS, Ellis SJ, et al; HF-ACTION Investigators. Efficacy and safety of exercise training in patients with chronic heart failure: HF-ACTION randomized controlled trial. JAMA 2009; 301: 1439-1450.

175. Tyni-Lenné R, Gordon A, Europe E, Jansson E, Sylvén C. Exer- 
cise-based rehabilitation improves skeletal muscle capacity, exercise tolerance, and quality of life in both women and men with chronic heart failure. J Card Fail 1998; 4: 9-17.

176. Working Group on Cardiac Rehabilitation \& Exercice Physiology and Working Group on Heart Failure of the European Society of Cardiology. Recommendations for exercise training in chronic heart failure patients. Eur Heart J 2001; 22: 125-135. (VII)

177. Demopoulos L, Yeh M, Gentilucci M, Testa M, Bijou R, Katz SD, et al. Nonselective beta-adrenergic blockade with carvedilol does not hinder the benefits of exercise training in patients with congestive heart failure. Circulation 1997; 95: 1764-1767. (V)

178. Forissier JF, Vernochet $\mathrm{P}$, Bertrand $\mathrm{P}$, Charbonnier $\mathrm{B}$, Monpère $\mathrm{C}$. Influence of carvedilol on the benefits of physical training in patients with moderate chronic heart failure. Eur J Heart Fail 2001; 3: $335-342$.

179. Jolly K, Taylor RS, Lip GY, Davies M, Davis R, Mant J, et al. A randomized trial of the addition of home-based exercise to specialist heart failure nurse care: The Birmingham Rehabilitation Uptake Maximisation study for patients with Congestive Heart Failure (BRUM-CHF) study. Eur J Heart Fail 2009; 11: 205-213. (A)

180. Smart N, Haluska B, Jeffriess L, Marwick TH. Exercise training in systolic and diastolic dysfunction: Effects on cardiac function, functional capacity, and quality of life. Am Heart $J$ 2007; 153: $530-536$.

181. Freimark D, Shechter M, Schwamenthal E, Tanne D, Elmaleh E, Shemesh $Y$, et al. Improved exercise tolerance and cardiac function in severe chronic heart failure patients undergoing a supervised exercise program. Int J Cardiol 2007; 116: 309-314.

182. Nishi I, Noguchi T, Iwanaga Y, Furuichi S, Aihara N, Takaki H, et al. Effects of exercise training in patients with chronic heart failure and advanced left ventricular systolic dysfunction receiving $\beta$-blockers. Circ J 2011; 75: 1649-1655.

183. Rodkey SM, Young J, Squires RW. Rehabilitation of patients with heart failure. In: Pashkow FJ, Dafoe WA, editors. Clinical cardiac rehabilitation. A cardiologist's guide, 2nd edn. Baltimore: Williams \& Wilkins, 1999.

184. Nishi I, Noguchi T, Furuichi S, Iwanaga Y, Kim J, Ohya H, et al. Are cardiac events during exercise therapy for heart failure predictable from the baseline variables? Circ J 2007; 71: 1035-1039.

185. Smart N, Fang ZY, Marwick TH. A practical guide to exercise training for heart failure patients. $J$ Card Fail 2003; 9: 49-58.

186. Shuichi T, Satoru S, Takeshi B, Hiroshi T, Naohiko A, Yoshio Y, et al. Predictors of left ventricular remodeling in patients with acute myocardial infarction participating in cardiac rehabilitation. Circ $J$ 2004; 68: 214-219.

187. Goto Y. Cardiac rehabilitation as heart failure therapy. Journal of the Japanese Association of Cardiac Rehabilitation (JJCR) 2008; 13: 273-277 (in Japanese).

188. McAlister FA, Stewart S, Ferrua S, McMurray JJ. Multidisciplinary strategies for the management of heart failure patients at high risk for admission: A systematic review of randomized trials. J Am Coll Cardiol 2004; 44: 810-819.

189. Rich MW, Beckham V, Wittenberg C, Leven CL, Freedland KE, Carney RM. A multidisciplinary intervention to prevent the readmission of elderly patients with congestive heart failure. $N$ Engl $J$ Med 1995; 333: 1190-1195.

190. Grady KL, Dracup K, Kennedy G, Moser DK, Piano M, Stevenson LW, et al. Team management of patients with heart failure: A statement for healthcare professionals from The Cardiovascular Nursing Council of the American Heart Association. Circulation 2000; 102: $2443-2456$

191. Vanhees L, Schepers D, Heidbüchel H, Defoor J, Fagard R. Exercise performance and training in patients with implantable cardioverter-defibrillators and coronary heart disease. Am J Cardiol 2001; 87: 712-715. (C)

192. Belardinelli R, Capestro F, Misiani A, Scipione P, Georgiou D. Moderate exercise training improves functional capacity, quality of life, and endothelium-dependent vasodilation in chronic heart failure patients with implantable cardioverter defibrillators and cardiac resynchronization therapy. Eur J Cardiovasc Prev Rehabil 2006; 13: $818-825$. (B)

193. Fitchet A, Doherty PJ, Bundy C, Bell W, Fitzpatrick AP, Garratt CJ. Comprehensive cardiac rehabilitation programme for implantable cardioverter-defibrillator patients: A randomised controlled trial. Heart 2003; 89: 155-160. (B)

194. Conraads VM, Vanderheyden M, Paelinck B, Verstreken S, Blankoff I, Miljoen H, et al. The effect of endurance training on exercise capacity following cardiac resynchronization therapy in chronic heart failure patients: A pilot trial. Eur J Cardiovasc Prev Rehabil 2007; 14: 99-106.
195. Patwala AY, Woods PR, Sharp L, Goldspink DF, Tan LB, Wright DJ. Maximizing patient benefit from cardiac resynchronization therapy with the addition of structured exercise training: A randomized controlled study. J Am Coll Cardiol 2009; 53: 2332-2339.

196. Corrà U, Giordano A, Bosimini E, Mezzani A, Piepoli M, Coats AJ, et al. Oscillatory ventilation during exercise in patients with chronic heart failure: Clinical correlates and prognostic implications. Chest 2002; 121: 1572-1580.

197. Corrà U, Pistono M, Mezzani A, Braghiroli A, Giordano A, Lanfranchi $\mathrm{P}$, et al. Sleep and exertional periodic breathing in chronic heart failure: Prognostic importance and interdependence. Circulation 2006; 113: 44-50.

198. Kasai T, Usui Y, Yoshioka T, Yanagisawa N, Takata Y, Narui K, et al; JASV Investigators. Effect of flow-triggered adaptive servoventilation compared with continuous positive airway pressure in patients with chronic heart failure with coexisting obstructive sleep apnea and Cheyne-Stokes respiration. Circ Heart Fail 2010; 3: $140-148$. (B)

199. Toyama T, Seki R, Kasama S, Isobe N, Sakurai S, Adachi H, et al. Effectiveness of nocturnal home oxygen therapy to improve exercise capacity, cardiac function and cardiac sympathetic nerve activity in patients with chronic heart failure and central sleep apnea. Circ J 2009; 73: 299-304. (B)

200. Ueno LM, Drager LF, Rodrigues AC, Rondon MU, Braga AM, Mathias W Jr, et al. Effects of exercise training in patients with chronic heart failure and sleep apnea. Sleep 2009; 32: 637-647. (C)

201. Bolling SF, Pagani FD, Deeb GM, Bach DS. Intermediate-term outcome of mitral reconstruction in cardiomyopathy. J Thorac Cardiovasc Surg 1998; 115: 381-386; discussion 387-388. (B)

202. Le Tourneau T, de Groote P, Millaire A, Foucher C, Savoye C, Pigny P, et al. Effect of mitral valve surgery on exercise capacity, ventricular ejection fraction and neurohormonal activation in patients with severe mitral regurgitation. J Am Coll Cardiol 2000; 36: $2263-2269$.

203. Meurin P, Iliou MC, Ben Driss A, Pierre B, Corone S, Cristofini P, et al; Working Group of Cardiac Rehabilitation of the French Society of Cardiology. Early exercise training after mitral valve repair: A multicentric prospective French study. Chest 2005; 128: $1638-$ 1644. (C)

204. Tei C, Horikiri Y, Park JC, Jeong JW, Chang KS, Toyama Y, et al. Acute hemodynamic improvement by thermal vasodilation in congestive heart failure. Circulation 1995; 91: 2582-2590. (B)

205. Kihara T, Biro S, Imamura M, Yoshifuku S, Takasaki K, Ikeda Y, et al. Repeated sauna treatment improves vascular endothelial and cardiac function in patients with chronic heart failure. $\mathrm{J} \mathrm{Am} \mathrm{Coll}$ Cardiol 2002; 39: 754-759. (B)

206. Kihara T, Biro S, Ikeda Y, Fukudome T, Shinsato T, Masuda A, et al. Effects of repeated sauna treatment on ventricular arrhythmias in patients with chronic heart failure. Circ J 2004; 68: 1146-1151. (B)

207. Kubozono T, Miyata M, Hasebe J, Tei C. Efficacy and safety of combination of exercise training and Waon therapy in patients with chronic heart failure. Journal of Japanese Association of Cardiac Rehabilitation (JJCR) 2011; 16: S114 (in Japanese). (B)

208. Kavanagh T, Yacoub MH, Mertens DJ, Kennedy J, Campbell RB, Sawyer P. Cardiorespiratory responses to exercise training after orthotopic cardiac transplantation. Circulation 1988; 77: 162-171. (B)

209. Ehrman J, Keteyian S, Fedel F, Rhoads K, Levine B, Shepard R. Ventilatory threshold after exercise training in orthotopic heart transplant recipients. J Cardiopulm Rehabil 1992; 12: 126-130. (C)

210. Tegtbur U, Busse MW, Jung K, Pethig K, Haverich A. Time course of physical reconditioning during exercise rehabilitation late after heart transplantation. J Heart Lung Transplant 2005; 24: 270-274. (B)

211. Haykowsky M, Riess K, Figgures L, Kim D, Warburton D, Jones $\mathrm{L}$, et al. Exercise training improves aerobic endurance and musculoskeletal fitness in female cardiac transplant recipients. Curr Control Trials Cardiovasc Med 2005; 6: 10. (B)

212. Kobashigawa JA, Leaf DA, Lee N, Gleeson MP, Liu H, Hamilton $\mathrm{MA}$, et al. A controlled trial of exercise rehabilitation after heart transplantation. N Engl J Med 1999; 340: 272-277. (A)

213. Nakatani T. Rehabilitation for the patients with ventricular assist system. Japanese Journal of Artificial Organs 1996; 25: 889-896 (in Japanese). (C)

214. Makita S, Sato S, Sakurada K, Majima M, Kyo S. Rehabilitation for severe heart failure patients with left ventricular assist device implantation: A comparison of exercise capacity with patients after CABG. Journal of the Japanese Association of Cardiac Rehabilita- 
tion (JJCR) 2003; 8: 26-28 (in Japanese). (C)

215. Richenbacher WE, Seemuth SC. Hospital discharge for the ventricular assist device patient: Historical perspective and description of a successful program. ASAIO J 2001; 47: 590-595. (C)

216. Wilson SR, Givertz MM, Stewart GC, Mudge GH Jr. Ventricular assist devices the challenges of outpatient management. J Am Coll Cardiol 2009; 54: 1647-1659. (C)

217. Rhodes J, Curran TJ, Camil L, Rabideau N, Fulton DR, Gauthier NS, et al. Impact of cardiac rehabilitation on the exercise function of children with serious congenital heart disease. Pediatrics 2005; 116: $1339-1345$. (B)

218. Rhodes J, Curran TJ, Camil L, Rabideau N, Fulton DR, Gauthier NS, et al. Sustained effects of cardiac rehabilitation in children with serious congenital heart disease. Pediatrics 2006; 118: e586-e593, doi:10.1542/peds.2006-0264. (B)

219. Takken T, Hulzebos HJ, Blank AC, Tacken MH, Helders PJ, Strengers JL. Exercise prescription for patients with a Fontan circulation: Current evidence and future directions. Neth Heart J 2007; 15: $142-147$. (C)

220. Cumming GR. Maximal exercise capacity of children with heart defects. Am J Cardiol 1978; 42: 613-619. (B)

221. Strieder DJ, Mesko ZG, Zaver AG, Gold WM. Exercise tolerance in chronic hypoxemia due to right-to-left shunt. J Appl Physiol 1973; 34: 853-858. (B)

222. Driscoll DJ. Exercise rehabilitation programs for children with congenital heart disease: A note of caution. Pediatr Exerc Sci 1990 2: $191-196 .(\mathrm{C})$

223. Williams MA, Fleg JL, Ades PA, Chaitman BR, Miller NH, Mohiuddin SM, et al. Secondary prevention of coronary heart disease in the elderly (with emphasis on patients $>$ or $=75$ years of age): An American Heart Association scientific statement from the Council on Clinical Cardiology Subcommittee on Exercise, Cardiac Rehabilitation, and Prevention. Circulation 2002; 105: 1735 1743. (C)

224. Audelin MC, Savage PD, Ades PA. Exercise-based cardiac rehabilitation for very old patients ( $>$ or $=75$ years): Focus on physical function. J Cardiopulm Rehabil Prev 2008; 28: 163-173. (B)

225. Frengley JD, Sansone GR, Alba A, Uppal K, Kleinfeld J. Influence of age on rehabilitation outcomes and survival in post-acute inpatient cardiac rehabilitation. J Cardiopulm Rehabil Prev 2011; 31: 230-238. (C)

226. Witt BJ, Jacobsen SJ, Weston SA, Killian JM, Meverden RA, Allison $\mathrm{TG}$, et al. Cardiac rehabilitation after myocardial infarction in the community. J Am Coll Cardiol 2004; 44: 988-996. (C)

227. Suaya JA, Stason WB, Ades PA, Normand SL, Shepard DS. Cardiac rehabilitation and survival in older coronary patients. $J$ Am Coll Cardiol 2009; 54: 25-33. (C)

228. Hammill BG, Curtis LH, Schulman KA, Whellan DJ. Relationship between cardiac rehabilitation and long-term risks of death and myocardial infarction among elderly Medicare beneficiaries. Circulation 2010; 121: 63-70. (C)

229. Seki E, Watanabe Y, Shimada K, Sunayama S, Onishi T, Kawakami $\mathrm{K}$, et al. Effects of a phase III cardiac rehabilitation program on physical status and lipid profiles in elderly patients with coronary artery disease: Juntendo Cardiac Rehabilitation Program (J-CARP). Circ J 2008; 72: 1230-1234. (A)

230. Onishi T, Shimada K, Sato H, Seki E, Watanabe Y, Sunayama S, et al. Effects of phase III cardiac rehabilitation on mortality and cardiovascular events in elderly patients with stable coronary artery disease. Circ J 2010; 74: 709-714. (A)

231. Fiatarone MA, O'Neill EF, Ryan ND, Clements KM, Solares GR Nelson ME, et al. Exercise training and nutritional supplementation for physical frailty in very elderly people. N Engl J Med 1994; 330: 1769-1775. (A)

232. Taaffe DR, Duret C, Wheeler S, Marcus R. Once-weekly resistance exercise improves muscle strength and neuromuscular performance in older adults. J Am Geriatr Soc 1999; 47: 1208-1214. (A)

233. McMurdo ME, Millar AM, Daly F. A randomized controlled trial of fall prevention strategies in old peoples' homes. Gerontology 2000; 46: 83 -87. (A)

234. Brubaker PH, Moore JB, Stewart KP, Wesley DJ, Kitzman DW Endurance exercise training in older patients with heart failure: Results from a randomized, controlled, single-blind trial. J Am Geriatr Soc 2009; 57: 1982-1989. (A)

235. Smart NA, Steele M. The effect of physical training on systemic proinflammatory cytokine expression in heart failure patients: A systematic review. Congest Heart Fail 2011; 17: 110-114. (A)

236. Oerkild B, Frederiksen M, Hansen JF, Simonsen L, Skovgaard LT, Prescott E. Home-based cardiac rehabilitation is as effective as centre-based cardiac rehabilitation among elderly with coronary heart disease: Results from a randomised clinical trial. Age Ageing 2011; 40: 78-85. (A)

237. Mameletzi D, Kouidi E, Koutlianos N, Deligiannis A. Effects of long-term exercise training on cardiac baroreflex sensitivity in patients with coronary artery disease: A randomized controlled trial. Clin Rehabil 2011; 25: 217-227. (A)

238. Tsuji I, Tamagawa A, Nagatomi R, Irie N, Ohkubo T, Saito M, et al. Randomized controlled trial of exercise training for older people (Sendai Silver Center Trial; SSCT): Study design and primary outcome. J Epidemiol 2000; 10: 55-64. (A)

239. Giallauria F, Lucci R, De Lorenzo A, D’Agostino M, Del Forno D, Vigorito C. Favourable effects of exercise training on N-terminal pro-brain natriuretic peptide plasma levels in elderly patients after acute myocardial infarction. Age Ageing 2006; 35: 601-607. (A)

240. Eder B, Hofmann P, von Duvillard SP, Brandt D, Schmid JP, Pokan $\mathrm{R}$, et al. Early 4-week cardiac rehabilitation exercise training in elderly patients after heart surgery. J Cardiopulm Rehabil Prev 2010; 30: 85-92. (A)

241. Ades PA, Savage PD, Brochu M, Tischler MD, Lee NM, Poehlman ET. Resistance training increases total daily energy expenditure in disabled older women with coronary heart disease. J Appl Physiol (1985) 2005; 98: 1280-1285. (A)

242. Watanabe S. Major vessel surgery. Monthly Book Medical Rehabilitation 2004; 41: 25-30 (in Japanese). (C)

243. Guidelines for the Diagnosis and Treatment of Cardiovascular Diseases (2004-2005 Joint Working Groups Report). Guidelines for Diagnosis and Treatment of Aortic Aneurysm and Aortic Dissection (JCS 2006). Circ J 2006; 70(Suppl IV): 1569-1646 (in Japanese). (C)

244. Guidelines for the Diagnosis and Treatment of Cardiovascular Diseases (2010 Joint Working Groups Report). Guidelines for Diagnosis and Treatment of Aortic Aneurysm and Aortic Dissection (JCS2011). http://www.j-circ.or.jp/guideline/pdf/JCS2011_takamoto_h. pdf (in Japanese)

245. Watanabe S. Assessment of quality of life (QOL) of patients with acute dissecting aortic aneurysm after discharge. Journal of the Japanese Association of Cardiac Rehabilitation (JJCR) 2001; 6: 102-104 (in Japanese). (C)

246. Okita Y. Treatment of aortic aneurism and aortic dissection: Surgery for thoracic aortic aneurism. In: Yutani C, Matsuo $\mathrm{H}$, editors. Clinical picture and pathology of aortic aneurysm and aortic dissection. Tokyo: Igaku Shoin, 2004: 88-99 (in Japanese). (C)

247. Brooke BS, Habashi JP, Judge DP, Patel N, Loeys B, Dietz HC 3rd Angiotensin II blockade and aortic-root dilation in Marfan's syndrome. N Engl J Med 2008; 358: 2787-2795.

248. Kato M. Challenges and future of stent grafting. Journal of Clinical Rehabilitation 2011; 20: 740-748 (in Japanese).

249. Stahler C, Strandness DE Jr. Ankle blood pressure response to graded treadmill exercise. Angiology 1967; 18: 237-241. (C)

250. Origuchi H. Rehabilitation after abdominal aortic aneurysm surgery. Journal of Clinical Rehabilitation 2011; 20: 730-735 (in Japanese).

251. Guidelines for the Diagnosis and Treatment of Cardiovascular Diseases (2006 Joint Working Groups Report). Guidelines for Rehabilitation in Patients with Cardiovascular Disease (JCS2007). http://www.j-circ.or.jp/guideline/pdf/JCS2007_nohara_h.pdf (in Japanese).

252. Watanabe S, Izawa K, Kobayashi T, Kobayashi T, Yokoyama H, Kondo M, et al. The inhibiting factors of physical therapy in aortic dissection repair. Physical Therapy Japan 2005; 32: $72-76$ (in Japanese).

253. Kothmann E, Batterham AM, Owen SJ, Turley AJ, Cheesman M, Parry A, et al. Effect of short-term exercise training on aerobic fitness in patients with abdominal aortic aneurysms: A pilot study. $\mathrm{Br}$ J Anaesth 2009; 103: 505-510.

254. Watanabe S. Cardiac surgery and physical therapy for elderly or disabled patients. The Japanese Journal of Physical Therapy 2005; 39: $771-776$ (in Japanese). (C)

255. Nishigami K. IV: Rehabilitations for complications of aortic disease. J Jpn Soc Int Med 2010; 99: 87-91(in Japanese).

256. Schouten O, Boersma E, Hoeks SE, Benner R, van Urk H, van Sambeek MR, et al; Dutch Echocardiographic Cardiac Risk Evaluation Applying Stress Echocardiography Study Group. Fluvastatin and perioperative events in patients undergoing vascular surgery. $N$ Engl J Med 2009; 361: 980-989.

257. Yamaguchi N, Odagiri N, Matsumoto Y. Nursing according to clinical pathway. In: Yutani $\mathrm{C}$, Matsuo $\mathrm{H}$, editors. Clinical picture and pathology of aortic aneurysm and aortic dissection. Tokyo: Igaku Shoin, 2004: 131 - 160 (in Japanese). (C)

258. Norgren L, Hiatt WR, Dormandy JA, Nehler MR, Harris KA, 
Fowkes FG; TASC II Working Group. Inter-Society Consensus for the Management of Peripheral Arterial Disease (TASC II). $J$ Vasc Surg 2007; 45(Suppl S): S5-S67. (B)

259. Gardner AW, Poehlman ET. Exercise rehabilitation programs for the treatment of claudication pain: A meta-analysis. JAMA 1995; 274: $975-980$. (A)

260. Proceedings of the 4th Circulatory Exercise Study Group Meeting. Angiology 2009; 8: 107-133 (in Japanese).

261. Regensteiner JG, Steiner JF, Hiatt WR. Exercise training improves functional status in patients with peripheral arterial disease. $J$ Vasc Surg 1996; 23: 104-115. (B)

262. Hiatt WR, Hirsch AT, Regensteiner JG, Brass EP. Clinical trials for claudication: Assessment of exercise performance, functional status, and clinical end points: Vascular Clinical Trialists. Circulation 1995; 92: 614-621. (B)

263. Stewart KJ, Hiatt WR, Regensteiner JG, Hirsch AT. Exercise training for claudication. N Engl J Med 2002; 347: 1941-1951.

264. Hiatt WR. Medical treatment of peripheral arterial disease and claudication. $N$ Engl J Med 2001; 344: 1608-1621.

265. 2011 Writing Group Members; 2005 Writing Committee Members; ACCF/AHA Task Force Members. 2011 ACCF/AHA Focused Update of the Guideline for the Management of patients with peripheral artery disease (Updating the 2005 Guideline): A report of the American College of Cardiology Foundation/American Heart Association Task Force on practice guidelines. Circulation 2011; 124: 2020-2045.

266. Dormandy JA, Rutherford RB. Management of peripheral arterial disease (PAD). TASC Working Group. TransAtlantic Inter-Society Consensus (TASC). J Vasc Surg 2000; 31(1 Pt 2): S1-S296.

267. Ohta T, Sugimoto I, Hida K, Kato M, Takeuchi N, Hosaka M, et al. The prediction of the effects of exercise training in patients with intermittent claudication. Jpn J Vasc Surg 1998; 7: 455-460 (in Japanese). (C)

268. Borg GA. Psychophysical bases of perceived exertion. Med Sci Sports Exerc 1982; 14: 377-381.

269. Yasu T, Saito M, Momomura S. Exercise training for vascular diseases. Journal of the Japanese Association of Cardiac Rehabilitation (JJCR) 2008; 1: 39-42 (in Japanese).

270. Matsuo H, Honma S, Hayashi F, Tsushima N. Study on long-term prognosis and quality of life of patients with Buerger disease. The Journal of Japanese College of Angiology 1997; 37: 883-886 (in Japanese).

271. Narins CR, Zareba W, Moss AJ, Marder VJ, Ridker PM, Krone RJ, et al. Relationship between intermittent claudication, inflammation, thrombosis, and recurrent cardiac events among survivors of myocardial infarction. Arch Intern Med 2004; 164: 440-446.

272. McPhee JT, Hill JS, Eslami MH. The impact of gender on presentation, therapy, and mortality of abdominal aortic aneurysm in the United States, 2001-2004. J Vasc Surg 2007; 45: 891-899.

273. Guidelines for the Diagnosis and Treatment of Cardiovascular Diseases (2005-2008 Joint Working Groups Report). Guidelines for management of peripheral arterial occlusive diseases (JCS 2009). Circ J 2009; 73(Suppl III): 1507-1569 (in Japanese).

274. Thompson PD, Zimet R, Forbes WP, Zhang P. Meta-analysis of results from eight randomized, placebo-controlled trials on the effect of cilostazol on patients with intermittent claudication. Am J Cardiol 2002; 90: 1314-1319.

275. Lièvre M, Morand S, Besse B, Fiessinger JN, Boissel JP. Oral Beraprost sodium, a prostaglandin I(2) analogue, for intermittent claudication: A double-blind, randomized, multicenter controlled trial. Beraprost et Claudication Intermittente (BERCI) Research Group. Circulation 2000; 102: 426-431.

276. Matsuo H, Shigematsu H. Effects of the 5-HT2A Antagonist Sarpogrelate on Walking Ability in Patients with Intermittent Claudication as Measured Using the Walking Impairment Questionnaire. Ann Vasc Dis 2008; 1: 102-110.

277. Scheffler P, de la Hamette D, Gross J, Mueller H, Schieffer H. Intensive vascular training in stage IIb of peripheral arterial occlusive disease: The additive effects of intravenous prostaglandin E1 or intravenous pentoxifylline during training. Circulation 1994; 90: $818-822$.

278. Mannarino E, Pasqualini L, Innocente S, Scricciolo V, Rignanese A, Ciuffetti G. Physical training and antiplatelet treatment in stage II peripheral arterial occlusive disease: Alone or combined? Angiology 1991 ; 42: $513-521$. (B)

279. Tsuchida $H$. Effects of exercise therapy: Home-based exercise therapy and drug treatment. In: Tada Y, editor. Practical treatment of arteriosclerosis obliterans. Tokyo: Bunkodo, 2004: 50-52 (in Japanese). (C)

280. Hayashi F. Treatment of claudication: Treat with exercise therapy.
Heart View 2003; 7: 1224-1249 (in Japanese).

281. Maejima Y, Yasu T, Ueba H, Kobayashi N, Hashimoto S, Kubo N, et al. Exercise after heparin administration: New therapeutic program for patients with-option arteriosclerosis oblitrans. Circ J 2005; 69: $1099-1104$.

282. Frasure-Smith N, Lespérance F, Talajic M. Depression and 18month prognosis after myocardial infarction. Circulation 1995; 91: 999-1005 (A).

283. Barefoot JC, Helms MJ, Mark DB, Blumenthal JA, Califf RM, Haney TL, et al. Depression and long-term mortality risk in patients with coronary artery disease. Am J Cardiol 1996; 78: 613-617. (A)

284. Denollet J, Sys SU, Stroobant N, Rombouts H, Gillebert TC, Brutsaert DL. Personality as independent predictor of long-term mortality in patients with coronary heart disease. Lancet 1996; 347: 417-421. (B)

285. Frasure-Smith N, Lespérance F, Talajic M. Depression following myocardial infarction: Impact on 6-month survival. JAMA 1993; 270: $1819-1825$. (B)

286. Barth J, Schumacher M, Herrmann-Lingen C. Depression as a risk factor for mortality in patients with coronary heart disease: A metaanalysis. Psychosom Med 2004; 66: 802-813. (A)

287. Blumenthal JA, Lett HS, Babyak MA, White W, Smith PK, Mark DB, et al; NORG Investigators. Depression as a risk factor for mortality after coronary artery bypass surgery. Lancet 2003; 362: 604-609. (B)

288. Carney RM, Blumenthal JA, Catellier D, Freedland KE, Berkman LF, Watkins LL, et al. Depression as a risk factor for mortality after acute myocardial infarction. Am J Cardiol 2003; 92: 1277-1281. (B)

289. Rosengren A, Hawken S, Ounpuu S, Sliwa K, Zubaid M, Almahmeed WA, et al; INTERHEART investigators. Association of psychosocial risk factors with risk of acute myocardial infarction in 11119 cases and 13648 controls from 52 countries (the INTERHEART study): Case-control study. Lancet 2004; 364: 953-962. (B)

290. Barefoot JC, Dahlstrom WG, Williams RB Jr. Hostility, CHD incidence, and total mortality: A 25 -year follow-up study of 255 physicians. Psychosom Med 1983; 45: 59-63. (A)

291. Iribarren C, Sidney S, Bild DE, Liu K, Markovitz JH, Roseman JM, et al. Association of hostility with coronary artery calcification in young adults: The CARDIA study: Coronary Artery Risk Development in Young Adults. JAMA 2000; 283: 2546-2551. (A)

292. Matthews KA, Owens JF, Kuller LH, Sutton-Tyrrell K, JansenMcWilliams L. Are hostility and anxiety associated with carotid atherosclerosis in healthy postmenopausal women? Psychosom Med 1998; 60: 633-638. (A)

293. Lespérance F, Frasure-Smith N. Depression in patients with cardiac disease: A practical review. J Psychosom Res 2000; 48: 379-391. (B)

294. Carney RM, Freedland KE. Depression, mortality, and medical morbidity in patients with coronary heart disease. Biol Psychiatry 2003; 54: 241-247. (B)

295. American Psychiatric Association. Diagnostic and Statistical Manual of Mental Disorders, 4th edn, Text Revision (DSM-IV-TR). Washington, DC: American Psychiatric Association, 2000.

296. Thombs BD, Bass EB, Ford DE, Stewart KJ, Tsilidis KK, Patel U, et al. Prevalence of depression in survivors of acute myocardial infarction. J Gen Intern Med 2006; 21: 30-38. (A)

297. Frasure-Smith N, Lespérance F. Recent evidence linking coronary heart disease and depression. Can J Psychiatry 2006; 51: 730-737. (B)

298. Rutledge T, Reis VA, Linke SE, Greenberg BH, Mills PJ. Depression in heart failure a meta-analytic review of prevalence, intervention effects, and associations with clinical outcomes. J Am Coll Cardiol 2006; 48: 1527-1537. (A)

299. Parashar S, Rumsfeld JS, Spertus JA, Reid KJ, Wenger NK, Krumholz $\mathrm{HM}$, et al. Time course of depression and outcome of myocardial infarction. Arch Intern Med 2006; 166: 2035-2043. (B)

300. Ruo B, Rumsfeld JS, Hlatky MA, Liu H, Browner WS, Whooley MA. Depressive symptoms and health-related quality of life: The Heart and Soul Study. JAMA 2003; 290: 215-221. (B)

301. Gehi A, Haas D, Pipkin S, Whooley MA. Depression and medication adherence in outpatients with coronary heart disease: Findings from the Heart and Soul Study. Arch Intern Med 2005; 165: $2508-$ 2513. (A)

302. Herridge ML, Linton JC. Psychosocial issues and strategies. In: American Association of Cardiovascular \& Pulmonary Rehabilitation, editor. AACVPR Cardiac rehabilitation resource manual: Promoting health and preventing disease. Champaign: Human Kinetics, 2005: 43-50. (A)

303. Ades PA, Waldmann ML, McCann WJ, Weaver SO. Predictors of 
cardiac rehabilitation participation in older coronary patients. Arch Intern Med 1992; 152: 1033-1035. (B)

304. Glazer KM, Emery CF, Frid DJ, Banyasz RE. Psychological predictors of adherence and outcomes among patients in cardiac rehabilitation. J Cardiopulm Rehabil 2002; 22: 40-46.

305. Frasure-Smith N, Lespérance F, Gravel G, Masson A, Juneau M, Talajic M, et al. Depression and health-care costs during the first year following myocardial infarction. J Psychosom Res 2000; 48: 471-478. (B)

306. Denollet J, Martens EJ, Nyklícek I, Conraads VM, de Gelder B. Clinical events in coronary patients who report low distress: Adverse effect of repressive coping. Health Psychol 2008; 27: $302-$ 308. (A)

307. Moussavi S, Chatterji S, Verdes E, Tandon A, Patel V, Ustun B. Depression, chronic diseases, and decrements in health: Results from the World Health Surveys. Lancet 2007; 370: 851 -858. (A)

308. Khan A, Leventhal RM, Khan SR, Brown WA. Severity of depression and response to antidepressants and placebo: An analysis of the Food and Drug Administration database. J Clin Psychopharmacol 2002; 22: 40-45. (B)

309. Fournier JC, DeRubeis RJ, Hollon SD, Dimidjian S, Amsterdam JD, Shelton RC, et al. Antidepressant drug effects and depression severity: A patient-level meta-analysis. JAMA 2010; 303: 47-53. (A)

310. American Psychiatric Association. Practice guideline for the treatment of patients with major depressive disorder, 3 rd edn. Arlington: American Psychiatric Association, 2010 Oct. (A)

311. Lichtman JH, Bigger JT Jr, Blumenthal JA, Frasure-Smith N, Kaufmann PG, Lespérance F, et al. Depression and coronary heart disease: Recommendations for screening, referral, and treatment: A science advisory from the American Heart Association Prevention Committee of the Council on Cardiovascular Nursing, Council on Clinical Cardiology, Council on Epidemiology and Prevention, and Interdisciplinary Council on Quality of Care and Outcomes Research: Endorsed by the American Psychiatric Association. Circulation 2008; 118: $1768-1775$. (A)

312. Lespérance F, Frasure-Smith N, Koszycki D, Laliberté MA, van Zyl LT, Baker B, et al; CREATE Investigators. Effects of citalopram and interpersonal psychotherapy on depression in patients with coronary artery disease: The Canadian Cardiac Randomized Evaluation of Antidepressant and Psychotherapy Efficacy (CREATE) trial. JAMA 2007; 297: 367-379. (B)

313. Berkman LF, Blumenthal J, Burg M, Carney RM, Catellier D, Cowan MJ, et al. Effects of treating depression and low perceived social support on clinical events after myocardial infarction: The Enhancing Recovery in Coronary Heart Disease Patients (ENRICHD) Randomized Trial. JAMA 2003; 289: 3106-3116. (A)

314. Burg MM, Lespérance F, Rieckmann N, Clemow L, Skotzko C, Davidson KW. Treating persistent depressive symptoms in postACS patients: The project COPES phase-I randomized controlled trial. Contemp Clin Trials 2008; 29: 231-240. (A)

315. Davidson KW, Rieckmann N, Clemow L, Schwartz JE, Shimbo D, Medina V, et al. Enhanced depression care for patients with acute coronary syndrome and persistent depressive symptoms: Coronary psychosocial evaluation studies randomized controlled trial. Arch Intern Med 2010; 170: 600-608.

316. Kanji N, White AR, Ernst E. Autogenic training reduces anxiety after coronary angioplasty: A randomized clinical trial. Am Heart $J$ 2004; 147: E10, doi:10.1016/j.ahj.2003.10.011. (A)

317. Milani RV, Lavie CJ. Impact of cardiac rehabilitation on depression and its associated mortality. Am J Med 2007; 120: 799-806. (B)

318. Callaghan P, Khalil E, Morres I, Carter T. Pragmatic randomised controlled trial of preferred intensity exercise in women living with depression. BMC Public Health 2011; 11: 465. (B)

319. Blumenthal JA, Babyak MA, Moore KA, Craighead WE, Herman $\mathrm{S}$, Khatri $\mathrm{P}$, et al. Effects of exercise training on older patients with major depression. Arch Intern Med 1999; 159: 2349-2356. (B)

320. Milani RV, Lavie CJ, Cassidy MM. Effects of cardiac rehabilitation and exercise training programs on depression in patients after major coronary events. Am Heart J 1996; 132: 726-732. (B)

321. Lavie CJ, Milani RV. Prevalence of anxiety in coronary patients with improvement following cardiac rehabilitation and exercise training. Am J Cardiol 2004; 93: 336-339. (B)

322. Burg MM, Barefoot J, Berkman L, Catellier DJ, Czajkowski S, Saab P, et al; ENRICHD Investigators. Low perceived social support and post-myocardial infarction prognosis in the enhancing recovery in coronary heart disease clinical trial: The effects of treatment. Psychosom Med 2005; 67: 879-888. (A)

323. Lett HS, Davidson J, Blumenthal JA. Nonpharmacologic treatments for depression in patients with coronary heart disease. Psychosom
Med 2005; 67(Suppl 1): S58-S62. (A)

324. Frasure-Smith N, Lespérance F, Gravel G, Masson A, Juneau M, Talajic M, et al. Social support, depression, and mortality during the first year after myocardial infarction. Circulation 2000; 101: 1919-1924. (A)

325. http://www.mhlw.go.jp/seisakunitsuite/bunya/kenkou_iryou/ iryouhoken/iryouhoken15/d1/2-22-5.pdf (in Japanese).

326. http://www.mhlw.go.jp/seisakunitsuite/bunya/kenkou_iryou/ iryouhoken/iryouhoken15/d1/2-22-7.pdf (in Japanese).

327. Alexopoulos GS, Reynolds CF 3rd, Bruce ML, Katz IR, Raue PJ, Mulsant BH, et al; PROSPECT Group. Reducing suicidal ideation and depression in older primary care patients: 24-month outcomes of the PROSPECT study. Am J Psychiatry 2009; 166: 882-890. (A)

328. Davidson PM, Cockburn J, Newton PJ, Webster JK, Betihavas V, Howes L, et al. Can a heart failure-specific cardiac rehabilitation program decrease hospitalizations and improve outcomes in highrisk patients? Eur J Cardiovasc Prev Rehabil 2010; 17: 393-402. (A)

329. Clark AM, Haykowsky M, Kryworuchko J, MacClure T, Scott J, DesMeules M, et al. A meta-analysis of randomized control trials of home-based secondary prevention programs for coronary artery disease. Eur J Cardiovasc Prev Rehabil 2010; 17: 261 -270. (A)

330. Throndson K, Sawatzky JA. Improving outcomes following elective percutaneous coronary intervention: The key role of exercise and the advanced practice nurse. Can J Cardiovasc Nurs 2009; 19: 17-24. (C)

331. Wood DA, Kotseva K, Connolly S, Jennings C, Mead A, Jones J, et al; EUROACTION Study Group. Nurse-coordinated multidisciplinary, family-based cardiovascular disease prevention programme (EUROACTION) for patients with coronary heart disease and asymptomatic individuals at high risk of cardiovascular disease: A paired, cluster-randomised controlled trial. Lancet 2008; 371: 1999_ 2012. (A)

332. Fridlund B. The role of the nurse in cardiac rehabilitation programmes. Eur J Cardiovasc Nurs 2002; 1: 15-18. (C)

333. Berra K. Does nurse case management improve implementation of guidelines for cardiovascular disease risk reduction? J Cardiovasc Nurs 2011; 26: 145 -167. (A)

334. Senaratne MP, Griffiths J, Mooney D, Kasza L, Macdonald K, Hare S. Effectiveness of a planned strategy using cardiac rehabilitation nurses for the management of dyslipidemia in patients with coronary artery disease. Am Heart $J$ 2001; 142: 975-981. (A)

335. Inglis SC, Pearson S, Treen S, Gallasch T, Horowitz JD, Stewart S. Extending the horizon in chronic heart failure: Effects of multidisciplinary, home-based intervention relative to usual care. Circulation 2006; 114: 2466-2473. (A)

336. Mittag O, China C, Hoberg E, Juers E, Kolenda KD, Richardt G, et al. Outcomes of cardiac rehabilitation with versus without a followup intervention rendered by telephone (Luebeck follow-up trial): Overall and gender-specific effects. Int J Rehabil Res 2006; 29: 295-302.

337. Myers J, Prakash M, Froelicher V, Do D, Partington S, Atwood JE. Exercise capacity and mortality among men referred for exercise testing. N Engl J Med 2002; 346: 793-801.

338. Itoh H, Ajisaka R, Koike A, Makita S, Omiya K, Kato Y, et al; Committee on Exercise Prescription for Patients (CEPP) Members. Heart rate and blood pressure response to ramp exercise and exercise capacity in relation to age, gender, and mode of exercise in a healthy population. J Cardiol 2013; 61: 71-78.

339. Tajima A, Ito H, Ishida T, Kokubo T, Sasazaki A, Nakamura T, et al. Estimated energy requirements for activities in daily living in Japan: Physical activities and exercise. Journal of Japanese Association of Cardiac Rehabilitation (JJCR) 2012; 17: 116-123 (in Japanese).

340. Miyaji M, Murakami H. A study on the validity of exercise guidance. Taiiku No Kagaku 2010; 60: 406-410 (in Japanese).

341. Nelson ME, Rejeski WJ, Blair SN, Duncan PW, Judge JO, King AC, et al. Physical activity and public health in older adults: Recommendation from the American College of Sports Medicine and the American Heart Association. Circulation 2007; 116: 1094-1105.

342. The Japanese Psychogeriatric Society. Psychiatric medicine for the elderly, revised edn: Overview. Tokyo: World Planning, 2009 (in Japanese). (B)

343. Takahashi M. Sleep disorder and health hazards due to overworking. Koshu Eisei 2007; 71: 302-306 (in Japanese).

344. Shima S, Hasegawa E. Consensus guidance on mental health for women. Tokyo; Nihon Hyoron Sha, 1999 (in Japanese).

345. Wisner KL, Parry BL, Piontek CM. Clinical practice: Postpartum depression. N Engl J Med 2002; 347: 194-199. 
346. Peppard PE, Young T, Palta M, Skatrud J. Prospective study of the association between sleep-disordered breathing and hypertension. N Engl J Med 2000; 342: 1378-1384.

347. Taylor DJ, Lichstein KL, Durrence HH. Insomnia as a health risk factor. Behav Sleep Med 2003; 1: 227-247.

348. Yamadera W, Itoh H, Inoue Y, Kanbayashi T, Tagaya H, Kamei Y. Guidelines for the diagnosis, treatment and collaborative management of sleep disorder. Suimin Iryo 2008; 2: 285-289 (in Japanese).

349. Uchiyama M, editor. Guidelines for management and treatment of sleep disorder. Tokyo: Jiho, 2002 (in Japanese).

350. Ueshima K, Mimura M, Nakagome K, Hirashima N, editors. Evidence-based psychiatry 2006-2007. Tokyo: Chugai Iyaku Sha; 2006 (in Japanese).

351. Morin CM, Bootzin RR, Buysse DJ, Edinger JD, Espie CA, Lichstein KL. Psychological and behavioral treatment of insomnia: Update of the recent evidence (1998-2004). Sleep 2006; 29: $1398-1414$

352. Haas DC, Foster GL, Nieto FJ, Redline S, Resnick HE, Robbins JA, et al. Age-dependent associations between sleep-disordered breathing and hypertension: Importance of discriminating between systolic/diastolic hypertension and isolated systolic hypertension in the Sleep Heart Health Study. Circulation 2005; 111: 614-621.

353. Kimura Y. The basic plan to prevent myocardial infarction in terms of lifestyle: Exercise therapy. Sogo Rinsho 2003; 52: 1475-1480 (in Japanese).

354. Sakai Y, Ikeda T, Goto S, Katsuki T, Takahashi T, Kita M. Collaboration between hospitals and exercise institutions to ensure smooth transition from recovery to maintenance phase cardiac rehabilitation (explanatory article). Journal of Japanese Association of Cardiac Rehabilitation (JJCR) 2010; 15: 130-133 (in Japanese).

355. Murakami J, Kimura Y. Collaboration between fitness clubs and medical institutions. The Journal of Japanese Society of Clinical Sports Medicine 2003; 11: 251-253 (in Japanese).

356. Yamaguchi $\mathrm{H}$, Katsuki $\mathrm{T}$. Maintenance-phase cardiac rehabilitation in health promoting institutions. Japanese Journal of Exercise Therapy 2009; 11: 20-23 (in Japanese).

357. Inoue M, Endo Y, Yamaguchi T, Ikeda K. Current status of cardiac rehabilitation in hospital-affiliated health promoting institutions. Japanese Journal of Exercise Therapy 2005; 7: 5-7 (in Japanese).

358. Saito H. Outline and current status of institutions defined in Article 42 of the Medical Care Law. Chiryo 2002; 84: 2974-2980 (in Japanese).

359. Kubo K, Ishihara S, Imai M, Murakami S, Yamamoto J, Shiozawa $\mathrm{E}$, et al. Collaboration in cardiac rehabilitation: Factors for continued exercise therapy in institutions for disease prevention defined in Article 42 of the Medical Care Law. Journal of Japanese Association of Cardiac Rehabilitation (JJCR) 2004; 9: 34-36 (in Japanese).

360. Tashiro T. Significance of collaboration pathways in local healthcare planning. Chiryo 2008; 90(Suppl 3): 707-714 (in Japanese).

361. Nagayama M, Tashiro T. Clinical pathways for collaborative practice for acte myocardial infarction in the Sakakibara Heart Institute. Chiryo 2008; 90(Suppl 3): 968-977 (in Japanese).

362. Takenaka K, Uechi H. Self-efficacy measures in physical activityand exercise-related studies. Taiikugaku Kenkyu 2002; 47: 209229 (in Japanese).

363. Norris SL, Engelgau MM, Narayan KM. Effectiveness of self-

\section{Appendix}

Chair:

- Ryuji Nohara, Hirakata Kohsai Hospital

Members:

- Hitoshi Adachi, Department of Cardiology, Gunma Prefectural Cardiovascular Center

- Yoichi Goto, Department of Cardiovascular Medicine, National Cerebral and Cardiovascular Center

- Emiko Hasegawa, Department of Human Welfare, Seigakuin University

- Shunichi Ishihara, Bunkyo University, Faculty of Human Sciences

- Haruki Itoh, Sakakibara Heart Institute

- Yutaka Kimura, Health Science Center, Kansai Medical University Hirakata Hospital

- Kazuhira Maehara, Shirakawa Kosei General Hosptal

- Shigeru Makita, Department of Cardiac Rehabilitation, Saitama Inter- management training in type 2 diabetes: A systematic review of randomized controlled trials. Diabetes Care 2001; 24: 561-587.

364. Berkhuysen MA, Nieuwland W, Buunk BP, Sanderman R, Rispens $P$. Change in self-efficacy during cardiac rehabilitation and the role of perceived overprotectiveness. Patient Educ Couns 1999; 38: $21-32$.

365. Harris DE, Record NB. Cardiac rehabilitation in community settings. J Cardiopulm Rehabil 2003; 23: 250-259.

366. Ito $\mathrm{H}$. Frontline of maintenance-phase rehabilitation for myocardial infarction: Discussion on phase III cardiac rehabilitation. Journal of Japanese Association of Cardiac Rehabilitation (JJCR) 2006; 15: $738-744$ (in Japanese).

367. Kimura N, Maeda T, Ito H, Ibi Y, Kurihara M, Hasegawa E, et al. Exercise therapy for hypertension: An approach in an intra-corporate health support center. Journal of Japanese Association of Cardiac Rehabilitation (JJCR) 2010; 15: 306-309 (in Japanese).

368. Mizukura I, Tamura T, Kimura Y, Yu W. New Application of IEEE 11073 to Home Health Care. Open Med Inform J 2009; 3: 44-53.

369. Kimura Y, Iwasaka T, editors. Strategies to prevent coronary artery disease from 30 years of age: A new concept of coronary artery disease. Tokyo: Nakayama Shoten, 2006: 254-263 (in Japanese).

370. Conroy MB, Yang K, Elci OU, Gabriel KP, Styn MA, Wang J, et al. Physical activity self-monitoring and weight loss: 6-month results of the SMART trial. Med Sci Sports Exerc 2011; 43: $1568-$ 1574.

371. Patrick K, Raab F, Adams MA, Dillon L, Zabinski M, Rock CL, et al. A text message-based intervention for weight loss: Randomized controlled trial. J Med Internet Res 2009; 11: e1, doi:10.2196/ jmir.1100.

372. Dellifraine JL, Dansky KH. Home-based telehealth: A review and meta-analysis. J Telemed Telecare 2008; 14: 62-66.

373. Varnfield M, Karunanithi MK, Särelä A, Garcia E, Fairfull A, Oldenburg BF, et al. Uptake of a technology-assisted home-care cardiac rehabilitation program. Med J Aust 2011; 194: S15-S19.

374. Kohzuki M. Cardiac rehabilitation and health insurance treatment. Junkanki Naika 2011; 69: 267-274 (in Japanese).

375. Notifications from the Medical Economics Division, Health Insurance Bureau, MHLW regarding the revision of NHI reimbursement list in 2012. URL: http://www.mhlw.go.jp/seisakunitsuite/bunya/ kenkou_iryou/iryouhoken/iryouhoken15/index.html, last accessed on April 24, 2012 (in Japanese).

376. Georgiou D, Chen Y, Appadoo S, Belardinelli R, Greene R, Parides $\mathrm{MK}$, et al. Cost-effectiveness analysis of long-term moderate exercise training in chronic heart failure. Am J Cardiol 2001; 87: 984-988; A4. (A)

377. Yu CM, Lau CP, Chau J, McGhee S, Kong SL, Cheung BM, et al. A short course of cardiac rehabilitation program is highly cost effective in improving long-term quality of life in patients with recent myocardial infarction or percutaneous coronary intervention. Arch Phys Med Rehabil 2004; 85: 1915-1922. (A)

378. Southard BH, Southard DR, Nuckolls J. Clinical trial of an Internetbased case management system for secondary prevention of heart disease. J Cardiopulm Rehabil 2003; 23: 341-348. (A)

379. Simchen E, Naveh I, Zitser-Gurevich Y, Brown D, Galai N. Is participation in cardiac rehabilitation programs associated with better quality of life and return to work after coronary artery bypass operations? The Israeli CABG Study. Isr Med Assoc J 2001; 3: 399-403. (A)

national Medical Center, Saitama Medical University

- Hiroshi Matsuo, Matsuo Clinic

- Shin-ichi Momomura, Cardiovascular Medicine, Jichi Medical University Saitama Medical Center

- Haruki Musha, Department of Sports Medicine, St. Marianna University School of Medicine

- Masatoshi Nagayama, Department of Cardiology, Sakakibara Heart Institute

- Takeshi Nakatani, Department of Transplantation, National Cerebral and Cardiovascular Center

- Tomoyuki Takura, Department of Health Care Economics and Industrial Policy, Osaka University Graduate School of Medicine

- Kenji Ueshima, Department of EBM Research, Institute for Advancement of Clinical Reserch and Translational Science, Kyoto University Hospital

- Ken Watanabe, Department of Pediatrics, Kitano Hospital

- Sumio Yamada, Department of Rehabilitation Science, Nagoya University Graduate School of Medicine 
- Akira Yamashina, Department of Cardiology, Tokyo Medical University Hospital

Collaborators:

- Toshimi Ikegame, St.Luke's International Hospital Branch Clinic St. Luke's Medilocus

- Masahiro Kohzuki, Department of Internal Medicine and Rehabilitation Science, Tohoku University Graduate School of Medicine

- Eisaku Nakane, Heart Center, Kitano Hospital

- Hideki Origuchi, Department of Internal Medicine, Kyushu Kosei Nenkin Hospital

- Shinji Sato, Department of Sports and Health Science, Osaka Sangyo University

- Tetsuya Takahashi, Department of Physical Therapy, Tokyo Univer- sity of Technology

- Katsutoshi Tanaka, Department of Occupational Mental Health, Kitasato University Graduate School of Medical Sciences

- Nozomi Tanaka, Heart Center, Kitano Hospital

- Toshiko Yoshida, Miyagi University School of Nursing

Independent Assessment Committee:

- Nobutaka Doba, The Life Planning Center Foundation

- Tohru Izumi, Kojinkai Niigata Minami Hospital

- Hirofumi Kambara, Shizuoka General Hospital

- Muneyasu Saito, Saitama Memorial Hospital

- Chuwa Tei, Waon therapy research institute/Dokkyo Medical University

(The affiliations of the members are as of July 2013) 